HEINER KUHL

\title{
UMWELTRESSOURCEN ALS GEGENSTAND INTERNATIONALER VERHANDLUNGEN
}




\section{HEINER KUHL}

\section{UMWELTRESSOURCEN ALS GEGENSTAND INTERNATIONALER VERHANDLUNGEN}

Umweltsysteme wie die Atmosphäre, Flüsse, Seen und Meere machen vor nationalen Grenzen nicht halt. Das wird in Europa besonders deutlich. Daher treten eine Vielzahl grenzüberschreitender Umweltallokationsprobleme auf. Aufgrund der nationalen Souveränitätsrechte lassen sich diese Probleme nur durch eine internationale Zusammenarbeit befriedigend lösen. Das damit verbundene Verhandlungsproblem wird in dieser Abhandlung untersucht. Mit Hilfe spieltheoretischer Ansätze wird im Rahmen von 2-Länder-Modellen analysiert, wie sich alternative Ausgangs- und Randbedingungen auf institutioneller Ebene wie auch in den jeweiligen Umweltsystemen auf mögliche Verhandlungsergebnisse auswirken. Dabei stehen allokations- und verteilungstheoretische Fragestellungen im Vordergrund.

Heiner Kuhl wurde 1952 in Leverkusen geboren. Studium der Volkswirtschaftslehre von 1974 bis 1980 an der Universität Bonn. Von 1981 bis 1986 wissenschaftlicher Assistent am Lehrstuhl für Volkswirtschaftslehre, insbesondere Mikroökonomie der Universität Mannheim. 
Umweltressourcen als Gegenstand internationaler Verhandlungen 


\title{
STAATLICHE ALLOKATIONSPOLITIK IM MARKTWIRTSCHAFTLICHEN SYSTEM
}

\author{
Herausgegeben von
}

Heinz König, Hans-Heinrich Nachtkamp, Uirich Schlieper, Eberhard Wille

Band 23

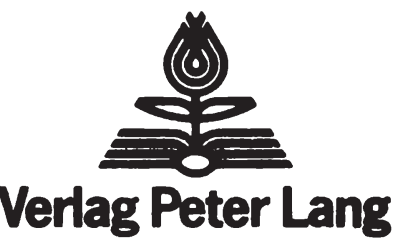

Frankfurt am Main - Bern - New York - Paris 


\section{HEINER KUHL}

\section{UMWELTRESSOURCEN \\ ALS GEGENSTAND \\ INTERNATIONALER \\ VERHANDLUNGEN}

Eine theoretische

Transaktionskostenanalyse

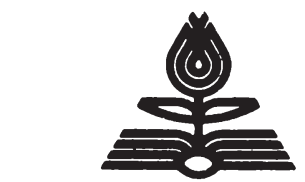

Verlag Peter Lang

Frankfurt am Main - Bern - New York - Paris 
CIP-Kurztitelaufnahme der Deutschen Bibliothek

\section{Kuhl, Heiner:}

Umweltressourcen als Gegenstand internationaler Verhandlungen : e. theoret. Transaktionskosten= analyse / Heiner Kuhl. - Frankfurt am Main , Bern ;

New York : Paris : Lang, 1987.

(Staatliche Allokationspolitik im marktwirtschaft= lichen System ; Bd. 23)

ISBN 3-8204-9931-8

NE: GT

Open Access: The online version of this publication is published on www.peterlang.com and www.econstor.eu under the international Creative Commons License CC-BY 4.0. Learn more on how you can use and share this work: http://creativecommons.org/ licenses/by/4.0.

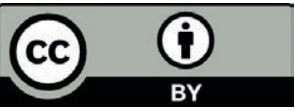

This book is available Open Access thanks to the kind support of ZBW - Leibniz-Informationszentrum Wirtschaft.

ISSN 0721-2860

ISBN 3-8204-9931-8

ISBN 978-3-631-75608-9 (eBook)

(C) Verlag Peter Lang GmbH, Frankfurt am Main 1987

Alle Rechte vorbehalten.

Das Werk einschließlich aller seiner Teile ist urheberrechtlich geschützt. Jede Verwertung außerhalb der engen Grenzen des Urheberrechtsgesetzes ist ohne Zustimmung des Verlages unzulässig und strafbar. Das gilt insbesondere für Vervielfältigungen, Übersetzungen. Mikroverfilmungen und die Einspeicherung und Verarbeitung in elektronischen Systemen.

Druck und Bindung: Weihert-Druck GmbH, Darmstadt 
Meinen Eltern 



\section{Vorwort}

Seit den siebziger Jahren sind grenzüberschreitende Umweltprobleme immer stärker in den Blickpunkt der Öffentlichkeit gerückt. Die Versalzung des Rheins, das durch Luftschadstoffe mitverursachte "Waldsterben" oder die erhöhte Strahlenbelastung in Europa nach dem Reaktorunfall in Tschernobyl sind allgemein bekannte Beispiele dafür. Es ist wohl allseitig akzeptiert, daß eine internationale Zusammenarbeit notwendig ist, um grenzüberschreitende Umweltprobleme in den Griff zu bekommen. Dennoch sind verbindliche institutionelle Regelungen bisher nicht geschaffen worden. Die Umweltpolitiken der betreffenden Staaten erfolgen weiterhin gröBtenteils unkoordiniert.

Die mit der internationalen Zusammenarbeit von staatlichen Behörden in diesem Bereich verbundenen Verhandlungsprobleme stehen im Mittelpunkt der vorliegenden Arbeit. In ihr wird mit Hilfe spieltheoretischer Ansätze im Rahmen von 2-Länder-Modellen untersucht, wie sich alternative Ausgangs- und Randbedingungen auf institutioneller Ebene wie auch in den jeweiligen Umweltsystemen auf mögliche Verhandlungsergebnisse auswirken. Dabei stehen allokations- und verteilungstheoretische Fragestellungen im Vordergrund.

Die Arbeit wurde im November 1986 von der Fakultät für Volkswirtschaftslehre und Statistik der Universität Mannheim als Dissertation angenommen.

Worte des Danks gelten zunächst meinem akademischen Betreuer Professor Dr. Klaus Conrad. Ohne seine Unterstützung hätte die Arbeit nicht entstehen können. Auch der Korreferent der Arbeit, Professor Dr. Horst Siebert, ist nicht ganz "unschuldig" daran. Das Grundmodell wurde in seinem Seminar an der Universität Konstanz vorgetragen. Von ihm und seinen Mitarbeitern erhielt ich eine Reihe von hilfreichen Anregungen und Hinweisen. Dafür bedanke ich mich sehr herzlich. 
Zu großem Dank bin ich Dr. Bernd Kosch verpflichtet. Er hat wesentliche Teile der Arbeit gelesen und konstruktiver Kritik unterzogen. In der stimulierenden "Müchener Diskussionsatmosphäre" wurde darüber hinaus manches verzwickt erscheinende Problem gelöst.

Nicht zuletzt möchte ich die wertvollen fachlichen Ratschläge meiner ehemaligen Kollegen erwähnen, erhalten zwischen "Tür und Angel" oder auch in stundenlangen Diskussionen. Dr. Helga Gebauer, Dr. Heinz Haller, Dr. Iris Henseler-Unger, Rainer Sauer und Dr. Ralph Unger sei herzlich dafür gedankt.

München, im Januar 1987

Heiner Kuhl 


\section{Inhaltsverzeichnis}

Seite

1. Einführung 1

1.1. Der Aufbau der Untersuchung 5

1.2. Zur umweltökonomischen Einordnung des Ansatzes $\quad 7$

2. Grundlegende Aspekte einer internationalen Zusammenarbeit zur koordinierten Nutzung gemeinsamer Umweltressourcen

2.1. Die ökologischen Einflußgrößen einer internationalen Zusammenarbeit

2.2. Die institutionellen und ökonomischen Einflußgrößen einer internationalen Zusammenarbeit

2.2.1. Bestimmungsgrößen von Wohlfahrtsgewinnen 20

2.2.2. Zur wohlfahrtsökonomischen Bedeutung von 21

Transaktionskosten

3. Die unkoordinierte Umweltnutzung in einer 2-Länder-Ökonomie

3.1. Das Modell einer 2-Länder-Ökonomie mit gemeinsamem Umweltsystem

3.1.1. Das gemeinsame Umweltsystem

3.1.2. Die Kosten-Nutzen-Bewertung des Einsatzes von Umweltgütern

3.2. Die nichtkooperative Nash-Lösung bei unkoordinierter Umweltnutzung

3.2.1. Das nichtkooperative Gleichgewicht

3.2.2. Die Auswirkungen von Parameteränderungen auf das Gleichgewicht 
4. Die statisch-axiomatische Lösung bei koordinierter Umweltnutzung

4.1. Das statische Verhandlungsmodell 50

4.1.1. Umweltkostenvorteile 51

4.1.2. Effiziente Umweltallokationen 56

4.1.3. Präferenzen der Regierungen $\quad 59$

4.2. Die verallgemeinerte Nash-Verhandlungslösung 60

4.2.1. Die Annahmen des verallgemeinerten Nash-Modells 61

4.2.2. Das verallgemeinerte Nash-Verhandlungsgleichgewicht 64

4.3. Die Grenzen des statisch-axiomatischen Nash-Modells 69

$\begin{array}{ll}\text { 4.4. Zusammenfassung } & 71\end{array}$

5. Die dynamisch-strategische Lösung bei koordinierter Umweltnutzung 75

5.1. Das dynamische Verhandlungsmodell 76

5.1.1. Verhandlungsstruktur 77

5.1.2. Zeitpräferenzen der Regierungen $\quad 80$

5.2. Das teilspielperfekte Nash-Gleichgewicht 84

5.3. Die Auswirkungen von Veränderungen der Verhandlungs- 98 bedingungen auf das Gleichgewicht

5.3.1. Einfluß der Zeitpräferenzen 100

5.3.2. Zeitlicher Abstand der Verhandlungsrunden 103

5.3.3. Einfluß von Risikoaversion 106

5.4. Die Auswirkungen von Veränderungen im Umweltsystem 109 auf das Gleichgewicht

5.4.1. Stabile Ausgangsbedingungen des Umweltsystems 111 über endliche Zeiträume

5.4.2. Stochastische Veränderungen der Ausgangsbedingungen des Umweltsystems

5.5. Zusammenfassung 
6. Die dynamisch-strategische Verhandlungslösung bei unvollständiger Information

6.1. Das dynamische Verhandlungsmodell bei unvollständiger Information

6.1.1. Unvollständige Information im Verhandlungsmodell

6.1.2. Informationstransfer im Verhandlungsmodell

6.2. Das teilspielperfekte Bayessche Nash-Gleichgewicht

7. Schlußbemerkung 



\title{
1. Einführung
}

\begin{abstract}
"The distinguishing problem in which environmental damage traverses international boundaries does not lie in the mix of programs that would work best if they could be adopted and enforced. The essential distinguishing feature, rather, lies in the difficulty in achieving any sort of effective international agreement and in seeing that it is carried out faithfully by all parties to that agreement."
\end{abstract}

(William J. Baumol $(1976,4))$

Natürlicherweise erstrecken sich ökologische Systeme wie die Erdatmosphäre, bestimmte Flüsse, Seen und Grundwasser sowie die Ozeane und deren Randmeere über die nationalen Grenzen souveräner Staaten hinweg oder liegen außerhalb davon. Dadurch sind diese nicht nur ökonomisch im Rahmen von Außenhandelsbeziehungen, sondern auch ökologisch miteinander verbunden. In der Folge davon wird ein großer Teil der bisher weitgehend freien Umweltgüter Luft, Wasser und Meeresressourcen von den Konsumenten und Produzenten mehrerer Länder gemeinsam genutzt. Dies ist in den vergangenen Jahrzehnten in immer größerem Umfang geschehen, so daß solche Güter heute relativ knapp sind. Aufgrund dessen werden wir mit einer Vielzahl von Umweltproblemen konfrontiert, die grenzüberschreitend sind oder sogar globale Ausmaße erreichen.

Deutlich zu erkennen sind solche Probleme in Mitteleuropa, da hier der Grad der ökologischen Vernetzung besonders hoch ist. Das durch weiträumig verteilte und abgelagerte Luftschadstoffe wie Schwefelkomponenten, Stickoxide, Schwermetalle und Ozon aus allen europäischen Industrieländern mitverursachte "Waldsterben"', die durch den Fallout

1) Siehe dazu das Sondergutachten des Rates von Sachverständigen zu Umweltfragen (1983). Dieser schätzt das "Waldsterben" als das derzeitig größte umweltpolitische Problem der Bundesrepublik ein. Ähnliche Schäden werden in kanadischen Waldgebieten beobachtet, die in der Abwindregion der US-amerikanischen und kanadischen Industrieregionen rund um die Großen Seen liegen. Vergleiche dazu Fay und Golomb (1983) sowie Forster (1984). 
des Reaktorunglücks im sowjetischen Kernkraftwerk Tschernobyl ausgelösten Schäden und die aufgrund der hohen Salzbelastung des Rheins durch die Abwässer der Kalibergwerke im Oberelsa $B$ eingeschränkte Trinkwassergewinnung bundesdeutscher und niederländischer Wasserwerke $^{21}$ sind wohlbekannte Beispiele für grenzüberschreitender Umweltprobleme.

Die Belastung der Stratosphäre mit chlorierten Fluorkohlenwasserstoffen, die einen Abbau der Ozon-Strahlenschutzschicht bewirken, ${ }^{31}$ oder die erhöhte Kohlendioxidbelastung der Atmosphäre, verantwortlich für Klimaveränderungen ("Treibhauseffekt") auf der Erde, 4) sind ebensolche Beispiele für globale Probleme. Aber auch der "Fischereikrieg" zwischen den Nordseeküstenländern im Zusammenhang mit der Beschränkung von Fischfangquoten in den isländischen Küstengewässern ist von seiner Natur her ein grenzüberschreitendes Problem bei der internationalen Nutzung von Umweltressourcen.

Nationale Souveränitätsrechte und fehlende überstaatliche Exekutivgewalt verhindern, daß deren Einsatz über nationale Grenzen hinweg reguliert werden kann. Somit können die jeweiligen Umweltbehörden grenzüberschreitende Umweltprobleme im nationalen Alleingang nur unvollständig oder garnicht lösen. ${ }^{5)}$ Es bleibt nur der Weg, den Ressourceneinsatz im Rahmen internationaler Zusammenarbeit gemeinsam zu regeln. ${ }^{61}$

2) Vergleiche dazu den Bericht "Warum das Salz immer noch in den Rhein gepumpt wird" in der FAZ vom 22.12.1985 und ebenso "Der versalzene Rhein" in der FAZ vom 24.5.1986.

3) Vergleiche dazu Gladwin, Ugelow und Walter (1982) und Ewe (1986).

4) Siehe dazu Kosobud und Daly (1984). Als Ursache wird auch hier, wie beim "Waldsterben", die umfangreiche Verbrennung fossiler Energieträger angesehen.

5) In manchen Situationen scheint eine Lösung auch nicht im nationalen Interesse zu liegen, wie in dem Fall eines grenzüberschreitenden Flußsystems, wenn ein Land als Oberlieger vorwiegend Verursacher von Umweltschäden bei den Unterliegern ist, ohne selbst wesentlich geschädigt zu werden.

6) Auf diesen Punkt weisen d'Arge (1974), (1976), Siebert (1978, 100ff.) und van Zele (1978) hin. 
In der Realität geschieht dies mittels bilateraler oder multilateraler zwischenstaatlicher Abkommen auf unterschiedliche institutionelle Weise. ${ }^{7)}$ Zum einen gibt es internationale Anstrengungen, die verschiedenen nationalen Rechtssysteme im Bereich des Privaten Haftungs- und Entschädigungsrechts sowie des Staatshaftungsrechts mittels äquivalenter Rechtsregeln einander anzugleichen und deren Anwendungsbereich im Umweltbereich auf internationale Fälle auszudehnen. ${ }^{81}$ Zum zweiten werden Abkommen angestrebt, die den Ressourceneinsatz direkt beschränken. Ein dritter gangbarer Weg ist die Einrichtung paritätisch mit Landesvertretern besetzter ständiger Kommissionen, die für ein grenzüberschreitendes Umweltsystem überstaatliche Regulierungskompetenz besitzen, ohne den nationalen Umweltbehörden direkt verantwortlich zu sein. ${ }^{91}$

Der meist langwierige Verlauf und die bisher sehr unergiebigen Ergebnisse realer internationaler Umweltverhandlungen sowie die Vielzahl noch unbewältigter oder nur oberflächlich gelöster internationaler Umweltprobleme deuten auf eine Reihe zu überwindender Hürden hin, um eine problemgerechte Lösung unter den jeweils gegebenen ökologischen, ökonomischen und institutionellen Ausgangsbedingungen zu erreichen. Das zeigt sich in den bisher relativ erfolglosen internationalen Bemühungen, die Luftverschmutzung in Europa zu reduzieren, ${ }^{101}$ sowie

7) Siehe dazu die Übersicht getroffener Abkommen zu grenzüberschreitenden Umweltproblemen in OECD $(1981,191 \mathrm{ff}$.$) .$

8) Vergleiche dazu OECD (1977) und Rest (1978) oder die neuere Diskussion im Rahmen der EG-Kommission zur Vollendung des Binnenmarktes.

9) Die International Joint Commission (IJC) zwischen den USA und Kanada hat entscheidenden Einfluß auf die Regulierung der Wasserverschmutzung in den Großen Seen und einer Reihe grenzüberschreitender Flüsse. Siehe dazu Mingst (1982). In Europa besitzen die zahlreichen ständigen Kommissionen, zuständig für die Belange einzelner ökologischer Systeme und Regionen, meist nur eine reine Informationsfunktion ohne eigene Entscheidungs- bzw. Regulierungskompetenz. Siehe dazu die Übersicht in OECD (1981, 138ff.).

10) Die 1979 unterzeichnete und 1983 in Kraft getretene ECE-Konvention, als einzig bedeutendes Abkommen zur europäischen Luftreinhaltung, enthält im wesentlichen nur Grundsatz- und Absichtserklärungen. Vergleiche dazu Prittwitz (1983a). 
dem zeitintensiven Bestreben insbesondere der Bundesrepublik und der Niederlande, daß die Salzfracht des Rheins verringert wird. ${ }^{11}$

Solange auf internationaler Ebene keine wirksame Zusammenarbeit zustande kommt, versucht einerseits jedes Land im nationalen Alleingang die inländischen Belastungen zu reduzieren. Die "Politik der hohen Schornsteine" mit dem Ziel, eine möglichst großflächige Verteilung schadstoffhaltiger Rauchgase zu erreichen, war bis vor wenigen Jahren in der Bundesrepublik und ist noch heute in Frankreich, Großbritannien und den Ostblocklandern dominierend. Andererseits sind aber auch Verhaltensweisen $\mathrm{zu}$ beobachten, die darauf abzielen, andere Staaten unter Druck zu setzen oder in einen politischen Zugzwang zu bringen. Die Ausdehnung nationaler Grenzen im Küstenbereich ("Nationalisierung") oder die derzeitige Vorreiterrolle der Bundesrepublik bei der Luftreinhaltung seien stellvertretend für solche Phänomene genannt.

Differierende Interessen oder strategisches Verhalten der an Umweltverhandlungen beteiligten Staaten, mangelnde "politische Dringlichkeit" einer gemeinsamen Lösung, mit der Zeit sich verändernde ökologische Umgebungsbedingungen, Informationsprobleme, ungenügende internationale Rechtssicherheit und auch die Organisation der Verhandlungen sind Einflußfaktoren, die möglicherweise eine Lösung von grenzüberschreitenden Umweltproblemen be- oder sogar verhindern können. Deren ökonomische Auswirkungen müssen im Zusammenhang zwischen den ökologischen und institutionellen Ausgangsbedingungen von Umweltverhandlungen, dem eigentlichen Verhandlungsproze $B$ und den daraus resultierenden, einigungsfähigen institutionellen Regelungen analysiert

11) Die Abkommen der "Internationalen Kommission zum Schutze des Rheins" sind ein gutes Beispiel dafür. Am 29. April 1963 gegründet, wurde am 3. Dezember 1976 als erstes das "Chemieabkommen" unterzeichnet, welches am 1.Februar 1979 in Kraft trat. Ebenfalls am 3. Dezember 1976 wurde das "Chloridabkommen" unterzeichnet, gemäß dem die Einleitung von Salzen aus der Produktion des Elsässischen KaliGruben in den Rhein durch den Aurbau einer Entsorgungstechnologie einschränkt werden soll. Dieses Abkommen trat erst am 5. Juli 1985 in Kraft, nachdem die Ratifizierung von der Französischen Regierung jahrelang verzögert worden war. In ca. 15 Jahren werden diese Kali-Lagerstätten ausgebeutet sein und mithin eine Entsorgung überflüssig werden. Vergleiche dazu Arnold (1984) und Uimwelt (7/1985, 37). 
werden. Dieser in der Literaturdiskussion zum großen Teil vernachlässigte Aspekt steht im Mittelpunkt der vorliegenden Arbeit. ${ }^{12)}$

\subsection{Der Aufbau der Untersuchung}

Der folgende Abschnitt führt zunächst in diejenigen Ansätze der umweltökonomischen Literatur ein, an welche diese Arbeit anknüpft. Anschliessend werden im Kapitel 2 die Auswirkungen spezieller ökologischer, ökonomischer und institutioneller Randbedingungen von internationalen Umweltverhandlungen allgemein skizziert. Den Hauptteil der Arbeit bilden in den Kapiteln 3 bis 6 Modelluntersuchungen, in denen die allokations- und verteilungstheoretischen Implikationen von Verhandlungen unter alternativen Randbedingungen mittels kooperativer und nichtkooperativer spieltheoretischer Ansätze behandelt werden.

Ausgangspunkt der Modelluntersuchungen ist eine in Kapitel 3 eingeführte 2-Länder-Ökonomie mit einem gemeinsamen Umweltsystem. Darin müssen sich zwei soziale Planer mit Hilfe einer Kosten-NutzenAnalyse für eine aus der Sicht jedes Landes optimale Umweltnutzung entscheiden. Die Formulierung der Umweltbedingungen erlaubt, ein- und gegenseitige Formen grenzüberschreitender Schadstofftransporte sowie die Common-Pool-Problematik internationaler Umweltgüter zu betrachten. Ebenso lassen sich durch unterschiedliche physische Ausgangsbedingungen und unterschiedliche Präferenzen der sozialen Planer unterschiedliche Randbedingungen des internationalen Umweltallokationsproblems simulieren. Dieses Modell wird zunächst benutzt, um daran in einem nichtkooperativen spieltheoretischen Modell die allokativen Folgen einer fehlenden internationalen Zusammenarbeit aufzuzeigen.

12) Die Schwerpunkte in der Literatur zu grenzüberschreitenden Umweltproblemen liegen einerseits darin, an Wohlfahrts- und Gerechtigkeitskriterien orientierte allgemeine Prinzipien zu formulieren, die als Basis einer internationale Zusammenarbeit dienen sollen. Andererseits wird die relative Vorteilhaftigkeit verschiedener organisatorischer Lösungen untersucht, d.h. unter welchen Bedingungen zum Beispiel ständige Kommissionen einer Fall-zu-Fall-Regelung vorzuziehen sind. Hier sind vor allem die Arbeiten im Rahmen der OECD-Diskussion zu grenzüberschreitenden Umweltproblemen zu nennen. Siehe dazu OECD (1974), (1976) und (1981). 
Anschließend wird in den Kapiteln 4 bis 6 diskutiert, welche allokativen Verbesserungsmöglichkeiten durch eine zwischenstaatliche Kooperation erreichbar sind. Zur Beschreibung der Verhandlungssituation auf einem vollkommenen Verhandlungsmarkt ohne Transaktionskosten dient in Kapitel 4 das kooperative Nash-Verhandlungsmodell in einer verallgemeinerten Form. Ein Verhandlungsmarkt mit potentiellen Transaktionskosten wird in den Kapiteln 5 und 6 mit Hilfe von dynamischen nichtkooperativen Verhandlungsmodellen dargestellt.

Verhandeln wird in dem nichtkooperativen Modelltyp als ein sequentieller und zeitintensiver Prozeß verstanden. Dieser impliziert für die Verhandlungsparteien ein strategisches, den Randbedingungen angepaßtes Verhalten. Die Interdependenzen zwischen den jeweiligen Randbedingungen und dem Ablauf des Verhandlungsprozesses sind entscheidend für die individuellen Transaktionskosten einer Verhandlungspartei im Verlauf der Verhandlungen. Die Modellierung des Verhandlungsproblems als ein nichtkooperatives Spiel, in dessen Regeln der Ablauf der Verhandlungen und eine mögliche Einigung genau festgelegt sind, erlaubt die Anwendung des in der Spieltheorie stärker eingeschätzten nichtkooperativen Nash-Gleichgewichtskonzepts. Dieses läßt im Gegensatz zu dem kooperativen Gleichgewichtskonzept zu, ein individuellrationales Verhalten und dessen Auswirkungen auf die Allokations- und Verteilungseigenschaften von Verhandlungsergebnissen zu beschreiben.

Die Verwendung zweier unterschiedlicher spieltheoretischer Konzepte dient dazu, unterschiedliche Formen individuellen Verhaltens darzustellen. Dabei ergibt sich zugleich die Möglichkeit, einen Überblick über die heute wesentlichen Ansätze in der Theorie der Verhandlungsspiele zu geben und deren Anwendungsgrenzen bei speziellen Fragestellungen aufzuzeigen.

Während in Kapitel 5 die Verhandlungssituation als ein Problem bei vollkommener Information und darüber hinaus allgemeiner Unsicherheit dargestellt wird, steht in Kapitel 6 eine Situation mit einseitiger asymmetrischer Information im Mittelpunkt. Einer der sozialer Planer ist hier 
nur unvollkommen über die Verhandlungsbedingungen des anderen informiert. Unter Umständen entsteht bei dem besser informierten Planer der Anreiz, die eigenen Bedingungen in persönlich vorteilhafter Weise falsch darzustellen, während der schlechter informierte Planer in diesem Fall den Anreiz hat, die Wahrheit zu erfahren. Im Modell wird davon ausgegangen, daß die Gewinnung der zusätzlichen Information Zeit und weitere Ressourcen erfordert. Unter dieser Prämisse wird für beide Planer die Entscheidung zwischen einem anreizverträglichen, d.h. einem die wahren Bedingungen aufdeckenden Verhalten, und einem anreizunverträglichen Verhalten als ein endogenes Trade-off-Problem betrachtet.

In Kapitel 7 werden die Ergebnisse abschließend bewertet und ein Ausblick auf weitere mögliche Anwendungsbereiche der vorliegenden Modellbetrachtung gegeben.

\subsection{Zur umweltökonomischen Einordnung des Ansatzes}

Dieser Abschnitt zielt zunächst darauf ab, den Untersuchungsgegenstand abzugrenzen und die Problemstellung der vorliegenden Arbeit in die umweltökonomische Literaturdiskussion einzubetten. Dabei wird im Überblick aufgezeigt, an welchen Stellen der Diskussion die Arbeit anknüpft und welche Aspekte in der Untersuchung unberücksichtigt bleiben.

Zur analytischen Beschreibung der internationalen Auswirkungen des nationalen Einsatzes von Umweltressourcen als öffentliches Konsumgut, als Aufnahmemedium für Schadstoffe, als Lieferant von Rohstoffen und als Standortfaktor ${ }^{131}$ ist eine regionalisierte Sichtweise des Umweltallokationsproblems sinnvoll. Auf diese Weise wird berücksichtigt, daß ökologische und ökonomische bzw. politische Systeme eine räumliche Ausdehnung besitzen und dabei die Ausdehnung ökologischer Systeme nicht notwendigerweise gleich derjenigen von ökonomisch-politischen Systemen ist.

13) Vergleiche zu den Funktionen der Umwelt Siebert (1978, 2), (1981, 29). 
Im Modell werden dazu ökonomische Systeme bzw. Subsysteme in Regionen aufgeteilt. Ökonomische Interdependenzen, die durch den Austausch von Gütern und Faktoren zwischen den Regionen bestehen, sowie solche ökologischer Art, werden darin mittels entsprechender funktionaler Beziehungen dargestellt. ${ }^{14)}$ In einem solchen Modell läßt sich jedes internationale Umweltproblem als Spezialfall eines regionalisierten Umweltallokationsproblems auffassen, in dem die Grenzen ökonomischer Regionen mit den nationalen Grenzen souveräner Staaten übereinstimmen.

Die Unterscheidung von ökologischen und ökonomischen Interdependenzen zwischen ökonomischen Regionen spiegelt sich in der Diskussion der speziellen Aspekte internationaler Auswirkungen des Einsatzes von Umweltressourcen wider. In der Literatur werden zwei weitgehend voneinander unabhängige Problemkreise abgegrenzt. Zum einen entstehen indirekte Auswirkungen, wenn nationale Umweltallokationsentscheidungen im Zusammenspiel mit der Güter- und Faktorallokation den internationalen Handel beeinflussen. Zum zweiten ergeben sich direkte Auswirkungen, wenn mehrere Länder ökologisch untereinander verbunden sind. Einen Überblick zu den damit verbundenen Fragen bekommt man bei Walter (1975), (1976), Baumol und Oates (1975) sowie Siebert (1978, $99 \mathrm{ff}$.).

Die theoretische Analyse der indirekten Auswirkungen verbindet Außenhandelsfragen mit Umweltfragen. Im Mittelpunkt stehen dabei die Zusammenhänge, die in einer arbeitsteiligen Welt zwischen dem Bestand bzw. den Zugriffsmöglichkeiten eines Landes auf Umweltressourcen existieren, dem Ausmaß staatlicher Eingriffe in deren Nutzung und den komparativen Preisvorteilen im Außenhandel.

Ist eine Ökonomie am Welthandel beteiligt, dann wirken sich die Handelsbeziehungen auf die Konsum- und Produktionsaktivitäten und

14) In den Arbeiten von Forsund (1972), Tietenberg (1974) werden solche Zusammenhänge im Kontext einer regionalisierten nationalen Umweltpolitik mit Steuern betrachtet. Einen Überblick über die theoretischen und institutionellen Probleme bekommt man bei Siebert, Waiter und Zimmermann (1980) sowie Siebert (1983a). 
damit auf die Umweltnutzung aus und umgekehrt. Umweltreguliernde Eingriffe in einem Land lösen "pekuniäre externe Effekte" in anderen Ländern aus, die aber über den Gleichgewichtsprozeß auf den Weltmärkten internalisiert werden. Somit ist ein internationaler Koordinierungsbedarf aus allokationstheoretischer Sicht unbegründet. Die Bedeutung der indirekten Auswirkungen liegt einerseits in den damit verbundenen Folgen für die Außenhandelsposition eines Landes und andererseits in den Rückkopplungsmechanismen über das Preissystem, die in einer optimalen nationalen Umweltpolitik berücksichtigt werden müssen. Diese Zusammenhänge werden in den Untersuchungen von Pethig (1976), Siebert (1977), (1979), Asako (1979), Siebert, Eichberger et. al. (1980) und Gronych (1980) herausgearbeitet. ${ }^{151}$

Im Mittelpunkt der vorliegenden Arbeit stehen die aus allokationstheoretischer Sicht gravierenderen direkten Auswirkungen, wenn aufgrund von ökologischen Verbindungen zwischen Ländern, deren Konsumenten und Produzenten bestimmte Umweltressourcen mit AllmendegutEigenschaften gemeinsam nutzen. ${ }^{16)}$ Solange dieses zwischenstaatlich unkoordiniert geschieht, besteht die Gefahr, daß der übermäßige Einsatz einer Umweltressource in einem Land ihre Verwendungsmöglichkeiten in anderen Ländern einschränkt.

Konkurrierende Verwendungen einer Umweltressource durch Konsumenten und Produzenten implizieren technologische externe Effekte ${ }^{171}$, die in

15) $\mathrm{Zu}$ den indirekten Auswirkungen zählen auch die der Umweltpolitik auf die Standortwahl emissionsintensiver Unternehmen. Man betrachtet dabei nicht die Mobilitåt von Gütern, Faktoren und Schadstoffen, sondern die Bewegungen schadensintensiver Aktivităten zwischen Regionen bzw. Ländern. Vergleiche beispielsweise Evans (1978) sowie Kneese und Williams (1980).

16) Die gleichen Probleme treten auch bei dem für die Informationsübertragung genutzten elektromagnetischen Wellenspektrum auf. Vergleiche zu externen Effekten durch Interferenzen und ihre Internalisierung, indem regionale Verfügungsrechte zugewiesen werden, Coase (1959) und insbesondere Minasian (1975).

17) Technologische externe Effekte sind im Entscheidungskalkül einer Wirtschaftseinheit unberücksichtigte und nicht über Preismechanismen ausgeglichene Wirkungen seiner Aktivitäten auf die anderer Wirtschaftseinheiten, die zu einer Differenz zwischen privaten und sozialen Kosten der Aktivität führen. Siehe dazu Heller und Starrett (1976). 
unserem speziellen, mehrere Länder tangierenden Fall eine internationale Dimension besitzen. Um diese vollständig internalisieren zu können, ist - wie schon zu Beginn aufgezeigt wurde - eine internationale Zusammenarbeit notwendig. Ansonsten bleiben die grenzüberschreitenden Effekte ungelöst bzw. werden möglicherweise durch nationale Regulierungseingriffe ("Politik der hohen Schornsteine") noch vergrößert.

In den Arbeiten von Markusen (1975a), (1975b), Tulkens (1979), Braden und Bromley (1981) sowie Arnold (1984) wird zur Beschreibung solcher Phänomene ein internationales öffentliches Gut unterstellt. ${ }^{18)}$ Anhand von statischen 2-Länder-Modellen weisen die Autoren nach, daß ein unkoordiniertes Verhalten nationaler Entscheidungsträger unter einer Cournot-Nash-Reaktionshypothese zu einer suboptimalen Allokation des internationalen Umweltguts führt, die aber durch eine Kooperation zwischen den Ländern verbessert werden kann. Markusen (1975a) zeigt zudem die weiteren Verbesserungen auf, die mit Hilfe von Transferzahlungen zwischen den Ländern realisiert werden können.

Soll die Kooperation wirksam zu einer Internalisierung der grenzüberschreitenden externen Effekte beitragen, müssen sich die autorisierten Behörden der betreffenden Staaten -in irgendeiner institutionellen Form - verpflichten, die inländische Nutzung der jeweiligen Umweltressource $\mathrm{zu}$ beschränken. Das damit verbundene Verhandlungsproblem zwischen den betreffenden Staaten wird in den o.g. Arbeiten noch nicht explizit berücksichtigt. Erst Munro (1979), in einem dynamischen Fischerei-Modell, und Pethig (1982), in einem statischen Modell für einen Fall gegenseitiger Umweltverschmutzung, untersuchen diese Problematik mit Hilfe des kooperativen Nash-Verhandlungsmodells.

Aus einem stärker institutionellen Blickwinkel wird das Kooperationsproblem zur Internalisierung von externen Effekten im Rahmen der Theorie der Verfügungsrechte (Property-Rights-Lehre) betrachtet. Diese

18) Vergleiche dazu auch Olson und Zeckhauser (1966), (1970), Breton (1970), Pauly (1970), Connolly (1972), Sandler (1975), Kiesling (1976) sowie Sandler und Culyer (1982a), (1982b). 
wurde entscheidend durch die Arbeiten von Coase (1960), Buchanan und Stubblebine (1962) sowie Demsetz (1964) geprägt. ${ }^{19)}$ Ihre prinzipielle Aussage ist, daß bei genügenden marktlichen Anreizen klar definierte Verfügungsrechte an einer Ressource, unabhängig von der Verteilung dieser Rechte in der Ausgangslage, hinreichend für eine effiziente Internalisierung externer Effekte auf Verhandlungsmärkten sind.

Diese Aussage, allgemein bekannt als "Coase-Theorem", hat eine in der Literatur kontrovers geführte Diskussion ausgelöst. Der Grund dafür liegt in den von Coase (1960) explizit und teils nur implizit unterstellten restriktiven Ausgangsannahmen. Entscheidend für das Ergebnis sind nach Hoffman und Spitzer (1982): Vollkommene Information, ein Verhandlungsmarkt ohne Transaktionskosten und ein vom gemeinsamen Interesse geleitetes Verhalten der Verhandlungsparteien. ${ }^{201}$ Coase geht nach Arrow (1979) und Samuelson (1985) danach implizit von einem Verhandlungsmodell aus, welches weitgehend die Merkmale eines traditionellen kooperativen Spiels besitzt. Diesem Spieltyp liegt die Verhaltensannahme zugrunde, daß sich die Verhandlungsparteien niemals auf Ergebnisse einigen, die eine allseitige Verbesserung ermöglichen, d.h. die Verhandlungsergebnisse sind in jedem Fall paretooptimal.

Damit klammert Coase alle Einflußgrößen aus, die Ursachen von Transaktionskosten sind und die be- oder sogar verhindern, daß entsprechende Verfügungsrechte an einer Ressource effizient getauscht bzw. zugewiesen werden können. Die Auswirkungen eines eigennützigen, strategischen Verhaltens der Verhandlungsparteien und jegliche Unvollkommenheiten des Verhandlungsmarktes bleiben unberücksichtigt. Existieren beispielsweise mehrere effiziente Ergebnisse, ist es bei einem eigennützigen Verhalten fraglich, ob sofort eine Einigung über eines von

19) Einen zusammenfassenden Überblick über diesen Problemkreis geben Furobotn und Pejovich (1972); neuere Arbeiten zu Umweltproblemen sind im dem von Wegehenkel (1981) herausgegebenen Konferenzband zu finden.

20) Hoffman und Spitzer (1982) geben neben dem systematischen Überblick über die expliziten und impliziten Voraussetzungen des Coase-Theorems zugleich eine Übersicht zu der Literatur, die Auswirkungen auf das Coase-Ergebnis untersucht, wenn einzelne Annahmen erweitert werden. 
ihnen erzielt wird oder ein zeit- und ressourcenverschwendendes Handeln darüber beginnt. Damit stellt sich die Frage, inwieweit der eigentliche Verhandlungsproze $B$ und die weiteren, Transaktionskosten verursachenden Randbedingungen entscheidend für die Dauer und das mögliche Ergebnis von Verhandlungen sind.

Geht man davon aus, daß eine bestimmte ausgehandelte Lösung für jede Verhandlungspartei unter den gegebenen Randbedingungen mit einem bestimmten $\mathrm{Ma} B$ an Transaktionskosten verbunden ist, dann besteht das Problem, bei welcher Form und welchem Umfang von Transaktionskosten noch ein effizientes Verhandlungsergebnis erreicht wird, eine Einigung auf eine ineffiziente Allokation bewirkt wird oder sogar keine Einigung zustande kommt. Ein zentraler Gedanke der "Neuen institutionellen Ökonomie"21" ist, daß bei einem eigennützigen Verhalten sich nur solche Institutionen bzw. institutionelle Regelungen durchsetzen können, die bei einer gegebenen Konstellation von Randbedingungen mit minimalen Transaktionskosten erreichbar sind. ${ }^{221}$

Die vorliegende Arbeit verbindet nun allokationstheoretische Ansätze aus der regionalisierten Umweltökonomie mit denjenigen aus der Institutionenökonomie; die neuere Theorie der Verhandlungsspiele ist dabei Mittel zum Zweck. In diesem Rahmen spielt der Transaktionskostenbegriff unter wohlfahrtstheoretischen Gesichtspunkten eine zentrale Rolle, die es zu erklären gilt. Die modelltheoretische Untersuchung in den Kapiteln 3 bis 6 beschränkt sich auf den 2-Länder-Fall mit einem gemeinsamen Umweltsystem, ein in der Außenhandeltheorie und der Umweltökonomie übliches Vorgehen. Ebenso bleiben dynamische ökologische Vorgänge, die in der Umweltökonomie von zentraler Bedeutung sind, bis auf einfach zu handhabende Randfälle außerhalb der Betrachtung. Hierzu sei auf die Arbeiten von Vogt (1981), Siebert (1985) und Gebauer (1985) verwiesen.

21) Vergleiche zu diesem $Z$ weig der Ökonomie die beiden Konferenzbände der Zeitschrift für die Gesamte Staatswissenschaft Band 140 März 1984 und Band 141 März 1985

22) Siehe dazu Barzel (1985), der die Transaktionskostenfrage im Zusammenhang mit Tauschvorgängen auf Produktmärkten analysiert. 


\section{Grundlegende Aspekte einer internationalen Zusam. menarbeit zur koordinierten Nutzung gemeinsamer Umweltressourcen}

Von grenzüberschreitenden Umweltproblemen betroffene Staaten sind nur dann interessiert, in diesem Bereich international zu kooperieren, wenn sie dadurch für ihr Land einen politischen oder wirtschaftlichen Vorteil erzielen können. Das ist in den vergangenen Jahren im Zusammenhang mit den internationalen Bemühungen deutlich geworden, die großräumigen Luftverschmutzungen in Europa und Nordamerika einzudämmen. Es waren vor allem die Länder mit einer "passiven" Emissionsbilanz wie Kanada, Norwegen und Schweden, von denen entsprechende Initiativen für eine internationale Emissionskontrolle ausgingen. Dagegen haben sich die Länder mit einer "aktiven" Emissionsbilanz wie Großbritannien, Frankreich und die USA weitgehend desinteressiert verhalten und verbindliche, umweltwirksame Regelungen verhindert."

Die Ursachen eines solchen eigennützigen Verhaltens sind im Souveränitätsprinzip und in der internationalen Rechtssituation begründet." Danach stellt sich die Suche nach lösungen für grenzüberschreitende Umweltexternalitaten als ein Verhandlungsproblem auf einem Markt dar, auf dem keinerlei übergeordnete Kontroll- und Regulierungsinstanz existiert. ${ }^{3}$ Eine gemeinsame Regelung kann immer nur im allseitigen und freiwilligen Einverständnis der beteiligten Staaten erreicht werden. Lösungen, die ökonomisch effizient und zugleich gerecht sind, aber dennoch Wohlfahrtsverluste für ein Land implizieren, können somit nicht gegen dessen Widerstand durchgesetzt werden.

1) Siehe Prittwitz (1984, 156ff.).

2) Ein solches Entscheidungsverhalten ergiht sich nach d'Arge (1974) daraus, daß das Souveränitätsprinzip einen Staat dazu verpflichtet, einmal erreichte ökonomische und politische Positionen des Landes nicht zu schwächen. Ein soiches "Rationalitäts postulat" erfüllt damit das Kriterium eines individuell-rationalen Verhaltens im Sinne der klassischen Entscheidungstheorie.

3) Die Lösung nationaler Cimweltprobleme über Verhandlungsmärkte, beispielsweise mit Hilfe von fungiblen Verschmutzungsrechten (Zertifikatslösung), schließt immer eine Regulierung der Märkte durch den Staat ein, zumindest um den Gesamtumfang der handelbaren Rechte zu beschränken. Vergleiche dazu Bonus (1981). 
In diesem Zusammenhang ist es die Aufgabe des Kapitels, wichtige ökologische, ökonomische und institutionelle Randbedingungen des zwischenstaatlichen Verhandlungsproblems im Überblick darzustellen. Daneben wird untersucht, inwieweit bei diesen Bedingungen unter Wohlfahrtsgesichtspunkten effiziente und zugleich gerechte Verhandlungslösungen erreicht werden können.

Im Abschnitt 2.1. wird dazu beschrieben, wie sich die spezifischen Eigenschaften ökologischer Systeme, die grenzüberschreitende Umweltprobleme verursachen, auf solche Regelungen auswirken. Die Aufgabe des Abschnitts 2.2. ist entsprechend, ökonomische und institutionelle Einflußgrößen zu erklären. Dabei gilt es, den wohlfahrtsökonomisch bedeutsamen Transaktionkostenbegriff zu erläutern und für die nachfolgende Modellanalyse zu präzisieren. Das ist notwendig, um aus theoretischer Sicht ein Verständnis für die Existenz, aber auch die Internalisierungsmöglichkeiten von externen Effekten auf Verhandlungsmärkten zu erhalten.

\subsection{Die ökologischen Einflußgrößen einer internationalen Zusammenarbeit}

Die natürliche geographische Ausdehnung und Lage eines ökologischen Systems, sein Potential an nutzungsfähigen Umweltressourcen sowie seine Regenerations- und Verteilungskräfte sind wichtige ökologische Einflußfaktoren des zwischenstaatlichen Verhandlungsproblems. Diese Größen sind entscheidend für die Interessenlagen zwischen den betreffenden Staaten. Sie beeinflussen das Ausma $B$ und die Form möglicher grenzüberschreitender Umweltexternalitäten und schränken damit die Möglichkeiten ein, auf einer zwischenstaatlichen Ebene zu einer gemeinsamen Regelung des Umweltressourceneinsatzes zu gelangen.

Je größer die Anzahl der Staaten ist, die durch die Ausdehnung und Lage eines ökologischen Systems eine Umweltressource gemeinsam nutzen, desto vielfältiger und komplexer ist das Netz der grenzüberschreitenden 
externen Effekte. Umso inhomogener sind folglich die Einzelinteressen dieser Staaten. Damit wachsen die Schwierigkeiten, eine institutionelle Regelungen zu finden, die einerseits allokative Verbesserungen bewirkt und andererseits genügend ökonomische Anreize für jeden Staat enthält, so daß sie allseitige Zustimmung findet. Je geringer das Potential eines ökologischen Systems an nutzungsfähigen Umweltressourcen und je geringer dessen Regenerationskräfte sind, umso dringlicher ist es meist, die bestehenden Schwierigkeiten rasch zu überwinden.

Betrachtet man zwei durch ein oder mehrere ökologische Systeme untereinander verbundene Länder, dann sind grenzüberschreitende externe Effekte prinzipiell in zwei verschiedenen Formen möglich. In der Form einseitiger externer Effekte schränkt der Einsatz der Umweltressource in einem Land ihre Nutzungsmöglichkeiten in einem anderen Land ein, aber nicht umgekehrt. Dagegen beeinflussen sich bei der Form gegenseitiger externer Effekte mehrere Länder wechselseitig.

Einseitige externe Effekte treten typischerweise bei ökologischen Systemen auf, die relativ hohe, vorwiegend in eine Richtung weisende innere Bewegungskräfte besitzen. Probleme ergeben sich meist dann, wenn Umweltressourcen in solchen Systemen als Schadstoffaufnahmemedium verwendet werden und es auf diese Weise zu einer einseitigen Verteilung von Umweltbelastungen kommt. Erstreckt sich das System in diesen sogenannten Oberlieger-Unterlieger-Situationen über nationale Grenzen hinaus, dann spricht man von transnationalen (grenzüberschreitenden) Umweltgütern. Das wohl bekannteste Beispiel für Umweltsysteme dieser Art sind Flußsysteme wie der Rhein mit seinen Nebenflüssen. Ebenso gilt das Umweltgut "Luft" als transnational, wenn in einem geographischen Raum eine weitgehend stabile Windrichtung vorherrscht.

Gegenseitige externe Effekte entstehen bei ökologischen Systemen, die keine einseitigen oder nur relativ geringe systematische innere Bewegungskräfte aufweisen. Systeme dieser Art sind meist besser durch die Vorstellung eines Common-Pools zu beschreiben, auf den mehrere Benutzer zugreifen und sich dabei gegenseitig beeinträchtigen. Ist ein solches ökologisches System grenzüberschreitend oder liegt außerhalb 
nationaler Grenzen, dann bezeichnet man die jeweiligen Ressourcen als internationale, bzw. wenn es sich über die ganze Erde ausdehnt, als globale Umweltgüter. Die Erdatmosphäre, die Ozeane und ihre Randmeere wie die Ostsee, aber auch Binnenseen, an die mehrere Länder angrenzen wie der Bodensee, sind typische Beispiele dafür. ${ }^{41}$

Eine strikt gegensätzliche Interessenlage entsteht bei einseitigen externen Effekten, wenn beispielsweise die Oberliegerstaaten eines Flusses Unterliegerstaaten durch eingeleitete Schadstoffe belasten. Der Oberlieger ist als Verursacher hierbei in einer "ökologischen Monopolposition". Er externalisiert Produktions- bzw. Entsorgungskosten zu Lasten der Unterlieger. Solange ihm keine eigenen Wohlfahrtsverluste entstehen, beispielsweise durch erhöhte Schäden, hat der Oberlieger ein Interesse, den gegenwärtigen Zustand zu erhalten oder sogar auszudehnen, während dem Unterlieger daran gelegen ist, daß die Belastungen verringert werden.

Die Interessengegensätze verstärken sich, wenn eine in Verhandlungen vorgeschlagene Regelung darauf abzielt, die Belastungen zu reduzieren, aber die Oberliegerstaaten die Kosten verstärkter Entsorgungsmaßnahmen, nach dem von der OECD vorgeschlagenen Verursacherprinzip alleine tragen sollen. ${ }^{5}$ Im Falle des vollständigen "Exports" der Umwelt. belastungen entstehen dem Oberlieger nur zusätzliche Kosten; der Unterlieger ist alleine Nutznießer. Insofern widerspricht das Verursacherprinzip in dieser Situation dem Eigeninteresse des Oberliegers. Beteiligen

4) Dazu lassen sich €benso "Luftbecken" zählen, d.h. räumlich begrenzte Gebiete mit geringen horizontalen Luftbewegungen in den unteren Schichten. Damit werden definitorische Abgrenzungsprobleme zwischen inter- und transnationalen Umweltgütern sichtbar. Vergleiche dazu Siebert $(1978,102)$.

5) In der Literatur werden im wesentlichen drei verschiedene allgemeine Prinzipien diskutiert: Das Verursacherprinzip geht davon aus, da $\beta$ bei einer Regelung der Verursacher die Entsorgungskosten alleine trägt, der Geschädigte dagegen die restlichen Schäden. Bei dem Geschädigtenprinzip muß der Geschädigte die verursachten Schäden und, wenn diese reduziert werden sollen, auch die entsprechenden Entsorgungskosten übernehmen. Die dazu gegensätzliche Alternative ist das Vollkostenprinzip, das auch als Zivilhaftungsprinzip bekannt ist. Hier muß der Verursacher Schäden wie auch mögliche Entsorgungskosten tragen. Vergleiche dazu beispielsweise Smets (1974) sowie Kneese und d'Arge (1984). 
sich die Unterliegerstaaten an den zusätzlichen Entsorgungskosten oder übernehmen diese gemäß dem Geschädigtenprinzip sogar vollständig, werden Interessengegensätze verringert bzw. abgebaut. Die Anreize vergrößern sich, daß einer solchen Lösung allseitig zugestimmt wird.

Läßt man sich im Gegensatz dazu von Gerechtigkeitsvorstellungen leiten, dann ist eine vom Verursacherprinzip getragene Lösung einer auf dem Geschädigtenprinzip aufbauenden Lösung sicherlich vorzuziehen. Im Fall von einseitigen grenzüberschreitenden externen Effekten kollidieren diese Vorstellungen aber mit ökonomischen Anreizüberlegungen, auf einem internationalen Verhandlungsmarkt eine Einigung zu erzielen. In dieser Situation wird besonders deutlich, daß eine solche Lösung nicht nur ein Allokationsproblem, sondern daneben ein ebenso bedeutendes Verteilungsproblem beinhaltet.

In dem Fall eines öknlogischen Systems, durch das gegenseitige externe Effekte ausgelöst werden, in der Common-Pool-Situation oder nach Prittwitz (1983a) ebenso in den Fällen von in verschiedenen Richtungen wirkenden einseitigen externen Effekten in unterschiedlichen ökologischen Systemen, sind die Interessengegensätze weniger stark ausgeprägt. Im Gegensatz zum Oberlieger bei einseitigen grenzüberschreitenden externen Effekten, kann keiner der Beteiligten eine "ökologische Monopolposition" aufbauen. Das Inland kann jeweils auf Aktionen des Auslands, die den ausländischen Ressourceneinsatz verändern, in gewissem $\mathrm{Maß}$ reagieren.

Durch diese gegenseitige Abhängigkeit sind die Eigeninteressen eher mit einem gemeinsamen Interesse gleichzusetzen. Neben dem Geschädigtenprinzip ist nach dem Grundsatz der Reziprozität das Verursacherprinzip mit den Eigeninteressen der Beteiligten vereinbar und hat entsprechende Chancen, in Verhandlungen akzeptiert zu werden. 


\subsection{Die institutionellen und ökonomischen Einflußgrößen einer internationalen Zusammenarbeit}

In der Realität arbeiten verschiedene Institutionen auf verschiedenen Ebenen international zusammen, um Allokationsprobleme bei inter- oder transnationalen Umweltressourcen zu losen. Das geschieht im wesentlichen auf der Ebene von Nachbarschaftsbeziehungen und auf der direkten zwischenstaatlichen Ebene. Auf der Nachbarschaftsebene zwischen Grenzregionen kooperieren regionale und lokale Behörden. Eine Zusammenarbeit findet hier fallweise. und im Umweltbereich ebenso in ständigen Kommissionen statt, die für die speziellen Belange einzelner grenzüberschreitender ökologischer Systeme bzw. Regionen eingerichtet wurden.

Entscheidender für Lösungen bei bedeutenden grenzüberschreitenden Unweltproblemen wie beispielsweise die angestrebte gemeinsame europäische Luftreinhaltepolitik oder die Festlegung internationaler Sicherheitsnormen für Kernkraftwerke bzw. Haftungregelungen bei möglichen Störfällen ist die Ebene der zwischenstaatlichen Beziehungen zwischen nationalen Regierungsstellen bzw. nationalen Umweltbehörden.

Die Gründe dafür sind: Erstens fällt ein großer Teil der grenzübergreifenden Umweltfragen von vornherein in den Kompetenzbereich der nationalen Behörden. Zumindest benötigen die regional oder lokal in Kommissionen getroffenen Vereinbarungen meist die Zustimmung der übergeordneten Behörden bzw. nationalen Parlamente. ${ }^{61}$ Zum zweiten bestehen für die nationalen Behörden natürliche zwischenstaatliche Kontakte durch die Zugehörigkeit von Staaten zu internationalen Organisationen und Einrichtungen wie beispielsweise zur Europäischen Gemeinschaft (EG) oder zur Internationalen Atomenergie-Organisation (IAEO). ${ }^{7}$

6) Vergleiche dazu Prittwitz (1983a), der internationale Umweltpolitik als einen Teil der Außenpolitik eines Landes ansieht.

7) Storsberg und Ercman (1980) skizzieren die Probleme, die durch fehlende Kompetenzen der regionalen Kommissionen entstehen. 
Rechtsgrundlage einer internationalen Zusammenarbeit bei grenzüberschreitenden externen Effekten sind die Regeln des Völkerrechts und des Internationalen Privaten und Öffentlichen Rechts. Diese sind im Hinblick auf eine internationale Kooperation in Umweltfragen allerdings sehr eingeschränkt. In der bisher praktizierten Zusammenarbeit fehlt - bis auf wenige Ausnahmen - der für eine wirksame internationale Umweltpolitik notwendige, rechtlich verbindliche Charakter. ${ }^{81}$

Im grenzüberschreitenden Rechtsverkehr existiert insbesondere kein eindeutiges Haftungsrecht, wodurch im Umweltbereich auf zwischenstaatlicher Ebene faktisch eine laissez-faire-Situation besteht. Demgemäß ist im Inland der einzelne Betroffene wie auch der Staat vor Wohlfahrtsverlusten, die durch eine Übernutzung von Umweltressourcen im Ausland entstehen und durch das grenzüberschreitende Umweltsystem "importiert" werden, weitgehend nicht geschützt. ${ }^{9 \prime}$

Dieser rechtlichen Ausgangssituation zufolge, müssen die betreffenden Behörden im Rahmen ihrer Zusammenarbeit zunächst Nutzungs- und Verfügungsrechte an den jeweiligen Umweltressourcen schaffen und können erst dann die Verteilung dieser Rechte untereinander aushandeln. Ökonomische Anreize, sich auf zwischenstaatlicher Ebene auf privatrechtlicher Basis verbindlich gegenseitig solche Rechte zuzuweisen, entstehen und wachsen in den Ländern mit der Größe der damit potentiell erzielbaren Wohlfahrtsgewinne. Deren theoretische Bestimmungsgrößen stehen im Mittelpunkt der beiden folgenden Unterabschnitte.

8) Vergleiche dazu Rest (1978) und Mayer-Tasch (1985). Das Prinzip 21 in der Schlußresolution der 1972 von den Vereinten Nationen veranstalteten Umweltkonferenz verpflichtet wohl die Staaten zu wechselseitiger Rücksichtnahme bei der Umweltnutzung. Der im Prinzip 22 der gleichen Resolution geforderten Weiterentwicklung des Internationalen Rechts in bezug auf Haftung und Entschädigung bei Umweltbelastungen, sind die Staaten bislang kaum gefolgt. Die in diesem Zusammenhang von der OECD formulierten Grundsätze über die Inländer-Ausländer-Gleichbehandlung bei Haftungsansprüchen vor inländischen Gerichten sind von deren Mitgliedern wohl akzeptiert, aber weitgehend noch nicht in die praktische Politik umgesetzt worden. Siehe dazu OECD (1977, 13ff.). Eine Ausnahme ist nach Rest (1978) die 1974 von den skandinavischen Ländern geschlossene "Nordic Environmental Protection Convention".

9) Dies hat der Reaktorunfall im sowjetischen Kernkraftwerk Tschernobyl wieder einmal sehr deutlich bestätigt. 


\subsubsection{Bestimmungsgrößen von Wohlfahrtsgewinnen}

Gehen wir von dem in der Realität weitgehend beobachteten eigennützigen Verhalten aus, läßt sich der bisher unkoordinierte, übermäßige Einsatz einer inter- oder transnationalen Umweltressource nur dann erfolgreich auf dem Weg über Verhandlungen gemeinsam regulieren, wenn alle an der Ressourcennutzung beteiligten Staaten daraus politische oder wirtschaftliche Vorteile ziehen können. ${ }^{101}$ Unter Kosten-NutzenGesichtspunkten betrachtet, setzt somit die Lösung von grenzuberschreitenden Umweltproblemen positive Wohlfahrtsgewinne für jedes Land voraus.

Die Quelle der Wohlfahrtsgewinne sind Effizienzzuwächse, die sich bei der Internalisierung der externen Effekte durch die Beschränkung des Umweltressourceneinsatzes auf ein paretosuperiores $\mathrm{Maß}$ erzielen lassen. Das Potential an Effizienzgewinnen hängt vor allem von den jeweiligen Konsum- und Produktionsbedingungen innerhalb des Einzugsbereichs eines ökologischen Systems ab. Das schließt die entsprechenden Entsorgungsmöglichkeiten und die Ausgangsbedingungen in Form der bisher geltenden nationalen umweltpolitischen Regulierungsmechanismen und -standards ein, wenn die Umweltressourcen des grenzüberschreitenden Systems als Schadstoffaufnahmernedium genutzt werden.

Bei der wohlfahrtsökonomischen Bewertung von Verhandlungslösungen sind aber ebenso die zur Realisierung der Effizienzgewinne notwendigen Aufwendungen von Zeit und anderen Ressourcen zu berücksichtigen. Ein solcher Ansatz unterstellt realistischerweise, daß Verhandlungsmärkte und gleichsam die administrativen Prozesse, mit denen die vereinbarten Regelungen durchgesetzt werden, unvollkommen sind und 'Transaktionskosten" verursachen.

10) Kneese und d'Arge (1984) haben an einigen Beispielen aufgezeigt, daß die möglichen Interessen von Staaten bei der Lösung von grenzüberschreitenden Limweltproblemen sehr unterschiedlicher Vatur sein können, so daß der Begriff des politischen und wirtschaftlichen Vorteils sehr weit gefaßt werden muß. Dadurch erhält das hierin verborgene Bewertungs- und Meßproblem eine zusätzliche Dimension. 
Die wesentlichen Ursachen von Unvollkommenheiten liegen in Friktionen und Informationsproblemen, denen Umweltbehörden bei der Organisation der Verhandlungen, in deren Verlauf sowie im Anschluß an den Verhandlungsproze $B$ bei der Durchsetzung von Regelungen ausgesetzt sind. Möglicherweise verursachen Behörden solche Friktionen auch selbst, um dadurch für sich strategische Vorteile zu gewinnen. Infolgedessen entstehen Transaktionskosten auf den Verhandlungsmärkten, wenn Informationen zu beschaffen sind und - wie in unserem Fall alternative Regelungen spezifiziert, ausgehandelt und administrativ durchgesetzt werden müssen. ${ }^{111}$

Bei dieser Form der Bewertung sind die Wohlfahrtsgewinne gleich der Differenz zwischen den bei einer Regelung des Ressourceneinsatzes erreichbaren Effizienzgewinnen und den zu ihrer Realisierung notwendigen Aufwendungen von Zeit und anderen Ressourcen. In diesem Zusammenhang haben Buchanan und Stubblebine (1962) den Begriff der "Pareto-Relevanz" geprägt. Demnach erscheinen Verhandlungen zur Internalisierung von externen Effekten nur dann wohlfahrtsökonomisch sinnvoll, wenn die Summe der für alle Staaten jeweils erreichbaren Effizienzgewinne größer als die Summe der individuellen Transaktionskosten ist.

\subsubsection{Zur wohlfahrtsökonomischen Bedeutung von Transaktionskosten}

Unterstellt man den Staaten, die an einem international koordinierten Einsatz inter- oder transnationaler Umweltressourcen interessiert sind, ein rationales Verhalten, dann können Unvollkommenheiten auf den jeweiligen Verhandlungsmärkten und der administrativen Prozesse eine mögliche Regulierung unvorteilhaft erscheinen lassen. In der wohlfahrtsökonomischen Diskussion grenzüberschreitender Umweltprobleme fällt somit dem Transaktionskostenbegriff ein zentrale Bedeutung zu.

11) Siehe dazu beispielsweise Windisch (1981), Bössmann (1982) oder North (1984). 
So inhaltlich allgemein wie der Transaktionskostenbegriff bisher abgegrenzt wurde, erlaubt er keinerlei Aussagen darüber, welche Allokationsund Verteilungswirkungen Transaktionskosten auf Verhandlungsmärkten nach sich ziehen. Es bleibt bei einem solchen "Sammelbegriff" unklar, ob Transaktionskosten per se effiziente Verhandlungsergebnisse verhindern, unbeeinflußt lassen oder möglicherweise sogar begünstigen. Auch der damit im Zusammenhang geprägte Begriff der Pareto-Relevanz trägt nicht zu einer genaueren Klärung bei. ${ }^{12)}$

Die Schwierigkeiten, die Transaktionskosten in einer wohlfahrtsökonomischen Analyse verursachen, resultieren zu einem großen Teil aus der inhaltlichen Komplexität dieses Kostenbegriffs. In unserem Fall läßt sich ein internationale gemeinsame Regulierung unter den jeweiligen Ausgangsbedingungen sicherlich mit Hilfe verschiedener Institutionen und Mechanismen marktlich und administrativ realisieren. Damit dürften auch Transaktionskosten unterschiedlicher Art und Höhe verbunden sein. Zum einen hängen diese in ihrer Art und Höhe davon ab, auf welche Weise der marktliche und der administrative Koordinationsprozeß organisiert wird. Zum anderen bedingen die Ursachen dieser Kosten selbst eine adäquate Organisation solcher Prozesse.

Danach bieten sich unter entsprechenden ceteris paribus Bedingungen zur Untersuchung grenzüberschreitender Umweltprobleme zwei verschiedene Vorgehensweisen an. ${ }^{13)}$

Im einen Fall können wir unter den gegebenen ökologischen Ausgangsbedingungen im Sinn einer Strukturanalyse untersuchen, welche institutionellen Organisations- und Verfahrensformen zur Lösung bestimmter grenzüberschreitender Umweltprobleme vorteilhaft sind. Dazu hat

12) Ein großer Teil dieser Probleme resultiert daraus, daß die Institutionenökonomie, beispielsweise bei der Erklärung, unter welchen Bedingungen ökonomische Aktivitäten über Märkte oder im Rahmen von Unternehmungen organisiert werden (Alchian und Demsetz (1972)), den Transaktionskostenbegriff uneinheitlich und durch mehr oder weniger umfassende Inhalte abgegrenzt hat.

13) Vergleiche dazu auch Bössmann (1982). 
Demsetz (1969) den "comparative institution approach" vorgeschlagen. Dabei werden alternative Organisations- sowie Verfahrensformen der Zusammenarbeit und ebenso alternative institutionelle Regelungen im Hinblick auf gewisse Normen untereinander verglichen und gemä $B$ den dabei entstehenden Abweichungen in eine Rangfolge gebracht. ${ }^{14)}$

Im anderen Fall - den wir weiter verfolgen - nimmt man die möglichen ökologischen, ökonomischen und institutionellen Ausgangsbedingungen und damit die marktlichen und administrativen Organisations- und Verfahrensformen als gegeben an. In einer Gleichgewichtsanalyse wird dann untersucht, wie sich die mit diesen Bedingungen verbundenen Transaktionskosten auf die Ergebnisse der Koordinationsprozesse auswirken.

Die Institutionenökonomie hat das aber bisher nur in unzureichendem Maß getan. Die bisherige Praxis, Transaktionskosten einfach als fixe Kosten zu betrachten oder als variable Kosten in Abhängigkeit von der möglichen institutionellen Regelung, erscheint als nicht problemgerechtes Vorgehen.

Bei einem reinen Fixkostenansatz würde man von der Überlegung ausgehen, daß der Koordinationsprozeß an sich kostenlos ist, aber die Organisation des Prozesses, die Beschaffung von Information oder auch die Durchsetzung einer vereinbarten Lösung den Einsatz einer bestimmten Menge an Ressourcen erfordert. ${ }^{15)}$

Transaktionskosten in diesem Sinne sind unabhängig vom Ablauf des Koordinationsprozesses und ebenso unabhängig von den daraus möglicherweise resultierenden Regelungen. Damit ist es schwierig einen

14) Hartje (1984) hat mit dieser Methode untersucht, inwieweit die Internationale Seerechtskonferenz der Vereinten Nationen als Gremium geeignet ist, um Zugangsbeschränkungen zur Meeresnutzung zu vergeben. Ähnlich geht Scott (1976), (1980) bei seinen Untersuchungen über grenzüberschreitende Umweltverschmutzungen vor.

15) Informationsbeschaffungskosten haben nach Arrow (1969) typischerweise Fixkostencharakter. Diese Einschätzung ist aber u.E nicht in jedem Fall gegeben. 
Unterschied zu "normalen" Fixkosten zu erkennen. Bei einer partialanalytischen Betrachtungsweise entstehen dadurch keinerlei Auswirkungen auf die Marginalbedingungen. Es stellt sich nur die Frage, ob diese Kosten größer oder kleiner als die möglichen Effizienzgewinne sind, d.h. ob paretoirrelevante oder paretorelevante Externalitäten bestehen. Beispielsweise dürften demnach in einer Ökonomie mit uneingeschränkten und reibungslosen Tauschmöglichkeiten für die nutzenmaximierenden Konsumenten und Produzenten nur paretoirrelevante Externalitäten zu beobachten sein. ${ }^{161}$

Ähnliche Interpretationsprobleme entstehen, wenn wir von Transaktionskosten als variablen Kosten ausgehen, die unabhängig vom Koordinationsprozeß nur von dem möglichen Ergebnis abhängen. Gemäß diesem Konzept entstehen bei einem Gütertausch Ressourcenverluste proportional zur Menge des getauschten Gutes.

Diese Form hat in der Geldtheorie zur Erklärung von Geld als Tauschmittel eine zentrale Bedeutung erlangt. Es wurde gezeigt, daß in einer Ökonomie mit mehreren Gutern im Gleichgewicht ausschließlich das Gut mit den niedrigsten Transaktionskosten als Tauschmittel verwandt wird. ${ }^{17}$ Dahlmann (1979) vergleicht diese Art daher mit gewöhnlichen Transportkosten, die aus dem Tauschvorgang aber nicht aus dem vorausgehenden Verhandlungsproze $\beta$ an sich resultieren. Insofern erscheint dieses Konzept im Kontext von Internalisierungsverhandlungen nicht sinnvoll.

Den Weg in die richtige Richtung hat Coase (1960) gewiesen, indem er sich in seiner Abgrenzung des Transaktionskostenbegriffs an den verschiedenen Phasen eines Verhandlungs- und Tauschprozesses orientiert hat:

16) In einem allgemeinen Gleichgewicht können Probleme nur durch die von Fixkosten ausgelösten Nichtkonvexitäten entstehen Vergleiche dazu Heller (1972).

17) Siehe dazu beispielsweise Hahn (1973), Kurz (1974) und Niehans (1975). Transaktionskosten eines solchen Typs werden auch von Blair und Kaserman $(1983,13 f f$. als Erklärungssgrößen für die vertikale Integration von Firmen verwendet. 
"In order to carry out a market transaction it is necessary to discover who it is that one wishes to deal with, to inform people that one wishes to deal and on what terms, to conduct negotiations leading up to a bargain, to draw up the contract, to untertake the inspection needed to make sure that the terms of the contract are being observed, and so on."

Danach entstehen Such- und Informationskosten, weil zum einen die potentiellen Verhandlungsparteien zueinander finden und die Verhandlungen organisieren müssen. Zum zweiten müssen sie sich gegenseitig über die jeweiligen physischen Ausgangsbedingungen und Präferenzen und damit gleichzeitig über mögliche einigungsfähige Lösungen informieren. Beispielsweise ist eines der wesentlichen Probleme bei Umweltproblemen ausreichende und verläßliche Information über ökologische Zusammenhänge und die entsprechenden Konsum- und Produktionsbedingungen.

Wir wissen heute noch relativ wenig über die genauen Abläufe in ökologischen Systemen. Für die Bestimmung optimaler Grenzwerte bei grenzüberschreitenden Umweltverschmutzungen ist es aber erforderlich, möglichst präzise Daten über die Emissions- und Immissionswirkungen, d.h. die Regenerations- und Verteilungskräfte in diesen Systemen, zu erhalten. ${ }^{181}$ Die Prozesse der Informationsgewinnung sind naturgemä $B$ sehr kosten- und zeitintensiv. Entsprechendes gilt für die jeweiligen Konsumund Produktionsbedingungen in den Einzugsbereichen der Systeme.

Im Verlauf der eigentlichen Verhandlungsphase entstehen den Ländern direkte Verhandlungs- und Entscheidungskosten, wenn die Behörden sich bei einer der verschiedenen möglichen Lösungen erst nach einem wiederum zeit- und ressourcenintensiven Aushandeln einigt. Zeitverluste und infolgedessen unrealisierte Effizienzgewinne verursachen aber auch die für internationale Konferenzen typischen, relativ großen Zeitabstände zwischen den einzelnen Konferenzrunden. Gleiches gilt für Zeitverluste,

18) Das Umweltprogramm der Vereinten Nationen (LNEP) hat hierzu beispielsweise entscheidendes geleistet. Im Rahmen dieses Programms sind europaweit vorhandene Meßstellen für Luftverschmutzungen weiter ausgebaut und zusätzliche neue Stellen eingerichtet worden, so daß heute ein flächendeckendes Meßraster für Luftverschmutzungen zur Verfügung steht. 
die aus der Ratifizierung der auf Kommissionsebene ausgehandelten Vereinbarungen durch nationale Behörden bzw. Parlamente resultieren.

Desweiteren verursachen administrative Prozesse Kosten, wenn die vereinbarte Regelung national erstmals durchgesetzt bzw. die bisherigen nationalen Regulierungsmechanismen und -standards an die neuen Erfordernisse angepaßt werden müssen. Zudem sind möglicherweise kostenintensive Kontrollmaßnahmen notwendig, um zu überprüfen, ob die vereinbarten Regelungen im Inland wie im Ausland eingehalten werden.

Der Vorteil einer solchen Abgrenzung des Transaktionskostenbegriffs ist, $\mathrm{da} B$ er sich an den Gegebenheiten des Koordinationsprozesses orientiert. Das wären im unserem Fall im wesentlichen die ökologischen, ökonomischen, organisatorischen und institutionell-rechtlichen Randbedingungen, die Präferenzen der beteiligten Staaten, ihr Verhalten unter den gegebenen Informationsbedingungen und die angestrebten institutionellen Regelungen. Der Nachteil besteht darin, daß sich Transaktionskosten dieser Art formal nicht einfach durch eine "Transaktionskostenfunktion" beschreiben lassen. Sie werden weitgehend implizit durch den tatsächlich ablaufenden marktlichen Koordinationsproze $\beta$ und den darauf folgenden Proze $B$ der administrativen Durchsetzung bestimmt.

In der nachfolgenden spieltheoretischen Modellanalyse wird gezielt der Koordinationsprozeß zwischen den von grenzüberschreitenden Umweltproblemen betroffenen Staaten herausgegriffen. Daran soll erklärt werden, welche Wechselwirkungen zwischen den ökologischen, ökonomischen und institutionellen Randbedingungen dieses Prozesses, den dabei entstehenden Transaktionskosten und den Ergebnissen bestehen. Die wohlfahrtökonomischen Implikationen, d.h. wie sich potentielle Transaktionskosten auf die Effizienz- und Verteilungseigenschaften einer Koordination auswirken, stehen im Mittelpunkt der Analyse. 


\section{Unkoordinierte Umweltnutzung in einer 2-Länder- Ókonomie}

In diesem Kapitel wird ein einfaches, partialanalytisches statisches Modell einer 2-Länder-Ökonomie entwickelt, in der zwei souveräne Länder jeweils Anteile an einer Umweltressource besitzen und diese gemeinsam nutzen. Die Ressource hat die Eigenschaften eines Allmendeguts, so daß die Gefahr einer Übernutzung besteht, wenn nicht die sozialen Planer jedes Landes ihren Einsatz auf ein wohlfahrtsoptimales Maß regulieren. Im Rahmen des Modells dient uns dafür als Anwendungsbeispiel die Umweltqualität in einem gemeinsamen Umweltsystem der beiden Länder.

Diese Ausgangssituation schließt ein internationales laissez-faire-Verhalten ein, wonach keinerlei völker- oder haftungsrechtliche Einschränkungen für den Einsatz der Umweltressource bestehen. Die Wahl optimaler staatlicher Eingriffe wird dadurch komplex, daß beide Länder jeweils nur auf nationaler Ebene die Ressourcennutzung regulieren können. Das Souveränitätsrecht läßt grenzüberschreitende Regulierungseingriffe nicht zu, so daß ein Land das andere nicht von der Nutzung ausschließen kann.

Zur Darstellung der grenzüberschreitenden Allokations- und Verteilungsprobleme betrachten wir jedes Land bis auf die gemeinsame Umweltressource als eine geschlossene Volkswirtschaft, in der private Konsumgüter produziert und konsumiert werden. In Teilbereichen der Konsumgüterproduktion fallen Schadstoffe als Kuppelprodukte an, deren unentsorgte Teile in Umweltsysteme am jeweiligen Produktionsstandort eingeleitet werden. Eines der Umweltsysteme in der Ökonomie ist in der Hinsicht grenzüberschreitend, daß Teilgebiete beider Länder zu seinem Einzugsbereich gehören. Somit können Emissionen in mehr oderr minder großem Anteil in das andere Land "exportiert" werden.

Dazu werden zunächst die beiden Länderökonomien durch die Umgebungsbedingungen in den inländischen Teilbereichen des gemleinsamen Umweltsystems und die Präferenzen der sozialen Planer spltzifiziert. Sodann wird gezeigt, daß die einseitige und zwischen den Lärldern un- 
koordinierte Regulierung zu einer ineffizienten Allokation der Umweltressource führt. Die Regulierungspolitik eines Landes bleibt bis auf Randfälle immer unvollständig, weil aufgrund der spezifischen Hoheitsrechte und Interessen nur die inländischen Einflußgrößen der inländischen Emissionen in das politische Kalkül des sozialen Planers eingehen. Zudem können auch nur die inländischen emittierenden Firmen reguliert werden. Die ausländischen Emissionsauswirkungen oder die aus der Sicht des Inlands unregulierbare Nutzung des inländischen Teils des Umweltsystems durch die Ausländer bleiben als externe Effekte bestehen.

Die Grundstruktur dieses Modells dient in den weiteren Kapiteln dazu, die allokativen Verbesserungen aufzuzeigen, die sich bei einer Koordination der Regulierungspolitiken zwischen den beiden Ländern erzielen lassen. Dabei werden insbesondere die Auswirkungen alternativer Rahmenbedingungen auf das Ergebnis einer international koordinierten Regulierung untersucht.

\subsection{Das Modell einer 2-Länder-Ökonomie mit gemeinsamem Umweltsystem}

Zur Modellierung der Länderökonomien gehen wir von einem für unsere Zwecke verfeinerten Kosten-Nutzen-Konzept aus. Modelle dieser Art werden standardmäßig in der Literatur benutzt, um Umweltprobleme darzustellen. So verwenden beispielsweise Muraro (1974) und Smets (1974) einen solchen Ansatz, um Oberlieger-Unterlieger-Situationen im Rahmen der OECD-Diskussion über Probleme bei grenzüberschreitenden Umweltprozessen zu beschreiben. Mit einem ähnlichen Ansatz untersuchen Roberts und Spence (1976), Kwerel (1977), Dasgupta, Hammond und Maskin (1980) sowie Repullo (1982) effiziente umweltregulierende Maßnahmen bei unvollkommener und asymmetrischer Information.

Der soziale Planer verfolgt in allen diesen Modellen das Ziel, durch seine umweltregulierenden Eingriffe in der Form von Emissionsstandards, 
Vergabe von Lizenzen oder durch eine entsprechende Steuerlösung eine Umweltallokation zu erreichen, die optimal im Sinne einer KostenNutzen-Analyse ist:" Die optimalen Emissionsbeschränkungen minimieren die Summe der aggregierten Umweltkosten, die sich aus der Summe der privaten Entsorgungskosten für schadstoffhaltige Kuppelprodukte und der Summe der bei einer Regulierung vermiedenen Schäden zusammensetzt.

Diese partialanalytische Betrachtungsweise hat den Vorteil, daß Interdependenzen zwischen Umwelt- und Konsumgüterallokation, sowie daran anknüpfende Verteilungsfragen zunächst als Sekundärprobleme außer acht gelassen werden. Somit lassen sich Umweltallokationsprobleme separat für jedes Umweltsystem analysieren. Auf diese Art reduziert sich die Modelluntersuchung auf die Allokation im gemeinsamen Umweltsystem, ohne daß die anderen Umweltsysteme der beiden Länder noch näher in die Betracht gezogen werden müssen. ${ }^{21}$

\subsubsection{Das gemeinsame Umweltsystem}

In den Einzugsbereichen des gemeinsamen Umweltsystems in den Ländern 1 und 2, im folgenden jeweils kurz als Umweltregion 1 bzw. 2 bezeichnet, existieren Firmen, bei deren Konsumgüterproduktion schadstoffhaltige Kuppelprodukte anfallen. Ein Teil dieser Kuppelprodukte wird mit adäquaten Entsorgungstechnologien beseitigt, ohne die Umwelt weiter zu belasten. Der nichtentsorgte Teil wird dagegen in das grenzüberschreitende Umweltsystem emittiert.

1) Vergleiche zur Anwendung des Kosten-Nutzen-Konzepts zur Lösung von Uimweltproblemen auch Mishan (1971, 119ff), Dasgupta und Pearce (1972), Siebert (1978, 68ff.), Freeman III (1979) sowie die breite Darstellung bei Halvorsen und Ruby (1981) und die Diskussion von Pearce (1976), (1977), Elliott und Yarrow (1977) und Smith (1977).

2) Im Hinblick auf das uns speziell interessierende grenzüberschreitende U'mweltallokationsproblem und seine Lösung durch eine internationale Kooperation, erscheint diese Vorgehensweise "praxisnäher" als eine Beschreibung des Problems in einem allgemeinen Gleichgewichtsmodell. 
Die Verteilungskräfte des Umweltsystems sorgen dafür, daß sich die an den verschiedenen Produktionsstandorten eingeleiteten Schadstoffe im System verteilen, abbauen oder auch an bestimmten anderen Orten anreichern. Diese Diffusions-, Advektions- und Assimilationsprozesse laufen in der Realität über Raum, Zeit und die verschiedenen Schadstoffe sehr komplex ab. ${ }^{3 /}$ Darüber hinaus sind in unserem Fall, durch die Aufspaltung des Umweltsystems in zwei Umweltregionen, noch zusätzliche Verteileffekte zwischen den Regionen zu beschreiben. Die Ansätze in der Umwelttheorie können diese Zusammenhänge in dieser Komplexität natürlich nicht abbilden und vereinfachen sie daher sehr.

Die für uns relevanten Regionenmodelle bauen weitgehend auf dem physikalischen Prinzip der Energie- und Masseerhaltung in einem geschlossenen System auf und stellen die Verteilungs- und Umwandlungsprozesse als ein dynamisches Problem von Immissionszunahmen durch Emissionserhöhungen und Immissionsabnahmen durch Assimilation dar. ${ }^{4}$

Wir vereinfachen diese Beschreibung für unsere $Z$ wecke noch stärker und gehen von einem direkten Zusammenhang zwischen aggregiertem Emissionsniveau in jeder Umweltregion und den dadurch in beiden Umweltregionen ausgelösten Immissionen aus. Damit unterstellen wir implizit, daß sich das Umweltsystem in einem stationären Gleichgewichtszustand befindet. Dieser ist dadurch gekennzeichnet, daß die assimilationsbedingte Immissionsabnahme gleich der emissionsbedingten Immissionszunahme ist. Nach kurzzeitigen Emissionserhöhungen bzw. -senkungen geht das System wieder unendlich schnell in seinen alten Gleichgewichts-

3) Unter Diffusion versteht man die Tendenz einer ursprünglich in einem Raumpunkt konzentrierten Anzahl von Partikeln, sich auf einer immer größeren Fläche rund um den Ursprungspunkt auszubreiten. Mit Advektion bezeichnet man dagegen die gezielte Verteilung der Partikel im Raum durch die zwangsweise Bewegung des Fließmediums in denen sie enthalten bzw. aufgelöst sind. Vergleiche dazu Okubo $(1980,8)$ und Gebauer (1985). Der Begriff der Assimilation beschreibt die Umwandlung systemfremder in systemnatürliche Stoffe.

4) Vergleiche dazu Basta und Bower (Hrsg.) (1982) mit einem Überblick über die Modelle und Methoden, die diesen Ansatz verwenden und dort insbesondere Basta und Moreau (1982, 23-120) sowie Muschett (1982, 389-546). 
zustand zurück. ${ }^{\text {5) }}$ Bei einer dauerhafter Änderung nimmt es sofort das neue Gleichgewichtsniveau an.

Zur Darstellung der Zusammenhänge zwischen den aggregierten Emissionsniveaus und den dadurch ausgelösten Immissionen in den beiden Umweltregionen unterstellen wir folgende Annahmen:

Erstens, die Immissionsbelastungen in den beiden Umweltregionen 1 und 2 mögen an jedem Ort, über die Zeit und die verschiedenen relevanten Schadstoffe meßbar und einheitlich bewertbar sein. Damit können beiden Umweltregionen vergleichbare, eindimensionale Belastungsindizes $s_{1}$ bzw. $s_{2}\left(s_{1}, s_{2} \in \mathbf{R}_{+}\right)$als $M a ß$ für das Immissionsniveau zugordnet werden. Die gleichen Bedingungen sollen für die über die einzelnen Firmen einer Region aggregierten Emissionsniveaus gelten. Auch diese seien somit in entsprechend genormten eindimensionalen Indizes $e_{1}$ und $e_{2}\left(e_{1}, e_{2} \in R_{+}\right)$ erfaßbar. ${ }^{61}$

Zweitens mögen sich die Diffusions-, Advektions- und Umwandlungszusammenhänge zwischen aggregierten Emissionen $e_{1}$ und $e_{2}$, sowie den dadurch in den Umweltregionen 1 und 2 ausgelösten Immissionen $s_{1}$ und $\mathbf{s}_{2}$, durch Umweltzustandsfunktionen $\mathrm{S}^{1}$ und $\mathbf{S}^{2}\left(\mathbf{S}^{1}, \mathbf{S}^{2}: \mathbf{R}^{2}{ }_{+} \rightarrow \mathbf{R}_{+}\right)$abbilden lassen. ${ }^{71}$ Vereinfachend gelte weiter, daß die Funktionen $\mathbf{S}^{1}$ und $\mathrm{S}^{2}$ linear und zeitunabhängig sind, sowie feste Koeffizienten besitzen, die die Eigenschaften des Umweltsystems approximativ beschreiben.

5) Vergleiche dazu Elliott und Yarrow (1977), Umweltgutachten (1978, 22),Vogt (1981, 14), der dieses als ökologischen Gleichgewichtszustand bezeichnet, und auch Gebauer $(1985,44)$.

6) Die in diesem und den nachfolgenden Kapiteln dargestellten Probleme lassen sich auch mit mehrdimensionalen Emissions- und Immissionsindizes erörtern. Den dazu notwendigen formalen Mehraufwand wollen wir aber vermeiden.

7) In dieser Arbeit werden Parameter, Variable sowie Funktionswerte mit kleinen lateinischen oder griechischen Buchstaben bezeichnet, Funktionen dagegen mit großen Buchstaben. Indizes werden an Parametern, Variablen und Funktionswerten als Subskript, an Funktionen als Superskript geschrieben. Das Subskript an Funktionen ist für die Kennzeichnung einer partiellen Ableitung nach dem entsprechenden Argument der Funktion vorbehalten. Ein Superskript an kleinen Buchstaben dient dagegen der Bezeichnung von Optimalwerten. 
Unter diesen Annahmen definieren wir Umweltzustandsfunktionen $\mathbf{S}^{1}$ und $\mathrm{S}^{2}$ mit folgenden Eigenschaften:

$$
\begin{aligned}
& S^{1}\left(e_{1}, e_{2}\right):=a_{1}\left[\left(1-\beta_{1}\right) e_{1}+\beta_{2} e_{2}\right], \\
& S^{2}\left(e_{1}, e_{2}\right):=a_{2}\left[\beta_{1} e_{1}+\left(1-\beta_{2}\right) e_{2}\right] .
\end{aligned}
$$

Darin beschreibt der Koeffizient $\beta_{i}, \beta_{i} \in[0,1]$, den prozentualen Anteil an Emissionen der Umweltregion $\mathrm{i}(\mathrm{i}=1,2)$ der in die andere Region "exportiert" wird. Der Koeffizient $\beta_{i}$ wird daher im folgenden als "Verteilungskoeffizient" bezeichnet. Der Koeffizient $a_{i}, a_{i} \geq 0$, ist ein $M a ß$ für den Anteil an den in einer Umweltregion verbleibenden und den von der ausländischen Region "importierten" Schadstoffen, die in einer Umweltregion als Immissionen wirksam werden. Ein $a_{i}$, für das gilt $0 \leq a_{i}<1$, deutet auf einen Abbau der Schadstoffe hin, ein $a_{i} \geq 1$ dagegen auf eine verminderte Assimilationskapazität und damit eine Anreicherung von Schadstoffen. ${ }^{81}$ Wir bezeichnen diesen Koeffizienten daher im folgenden als "Assimilationskoeffizienten". Es sei angemerkt, daß mit steigenden Assimilationskräften einer Umweltregion i der Koeffizient $a_{i}$ sinken muß.

Assimilations- und Verteilungskoeffizienten stellen im Modell exogene Einflußgrößen dar, mit denen spezifische Eigenschaften eines Umweltsystems simuliert werden können. Durch entsprechende Wahl der Verteilungskoeffizienten läßt sich zum einen eine Situation mit wechselseitigen Diffussionsvorgängen und zum anderen eine OberliegerUnterlieger-Situation darstellen. Eine Oberlieger-Unterlieger-Situation mit Umweltregion 1 als Oberlieger wäre durch die Wahl eines kleinen $\beta_{2}$ in Relation zu $\beta_{1}$ darstellbar. Das Common-Pool-Problem bei internationalen Umweltgütern könnte annähernd durch die Parameterkonstellation $\beta_{1}=\beta_{2}=0,5$ und $a_{1}=a_{2}$ beschrieben werden.

8) Realitätsnäher wäre eine Beschreibung dieser Koeffizienten durch Funktionen, die von der Zeit, dem Emissionsniveau sowie möglicherweise weiteren exogenen Größen abhăngen. Die Konstanz dieser Koeffizienten dient der Vereinfachung. Wir sind uns aber gerade im Hinblick auf den Assimilationskoeffizienten mit den damit verbundenen Problemen bewußt. Eine Anreicherung von Schadstoffen über die Zeit kann durch das "starre Gleichgewicht" nur sehr unvollkommen beschrieben werden, d.h. nur durch eine komparativ-statische Verănderung. 


\subsubsection{Die Kosten-Nutzen-Bewertung des Einsatzes von Umweltgütern}

In jedem Land i gebe es einen sozialen Planer, den wir im folgenden als Regierung i bezeichnen. Dessen umweltregulierende Aufgabe besteht darin, in den einzelnen Umweltsystemen des Landes bestimmte, unter Wohlfahrtsaspekten optimale Umweltqualitäten als Zielgrößen anzugeben und mit Hilfe einer Standard-, Steuer- oder auch Lizenzlösung durchzusetzen. Bei der Regulierung des grenzüberschreitenden Umweltsystems ist jede Regierung aufgrund der Souveränität der beiden Länder auf die inländische Umweltregion beschränkt. Sie trifft dazu auch nur solche Entscheidungen, die optimal im Sinne der inländischen Wohlfahrt sind.

Eine Bewertung der verschiedenen möglichen Umweltqualitäten nimmt jede Regierung vor, indem sie die Kosten der Umweltpolitik mit dem dabei jeweils erreichbaren Nutzen vergleicht. Die Kosten der Umweltpolitik bestehen dabei in den Kosten einer Verminderung des Schadstoffausstoßes, der Nutzen in den vermiedenen Schäden. Entsprechend dem vorgegebenen Verhaltensmuster orientiert sie sich, solange die beiden Regierungen nicht miteinander kooperieren, bei ihren umweltpolitischen Entscheidungen für das grenzüberschreitende Umweltsystem nur an den inländischen Kosten und Schäden.

Eine erste Entscheidungsgrundlage der Regulierungspolitik in der Umweltregion i sind somit die vom zulässigen aggregierten Emissionsniveau $e_{i}$ abhängigen privaten aggregierten Entsorgungskosten $c_{i}$ aller in deren Bereich emittierenden Firmen. ${ }^{9}$ Diese sinken natürlicherweise mit steigendem zulässigen Emissionsniveau $e_{i}$.

9) Wenn man davon ausgeht, daß Umweltregulierungsmaßnahmen effizient durchgesetzt werden können, dann ergibt sich für jede Emissionsnorm e die aggregierte Entsorgungkostenfunktion als Umhüllende aus der Summe der privaten individuellen Entsorgungskostenfunktionen. (Vergleiche dazu auch K werel (1977).) In diesem Fall würde sie für alle Firmen $\mathrm{j}$ durch die Lösung des Problems

$$
\operatorname{Min} \Sigma C^{i j}\left(e_{i j}\right) \quad \sum_{j} e_{i j}=\hat{e}_{i}<e_{i}{ }_{i} \quad(i=1,2)
$$

bestimmt, wobei $e$ das Fmissionsniveau der Firma $\mathrm{j}$ bei Regulierung, $\hat{e}_{i}$ die von der Regierung festgesetzte, aggregierte Emissionsnorm $e_{1}^{0}$ das aggregierte Emissionsniveau vor einer Regulierung und $\mathrm{C}^{-j}$ die Entsorgungskostenfunktion der Firma $\mathrm{j}$ ist. Sind diese fallend und konvex, dann besitzt die Umhüllende ebenfalls diese Eigen schaften. Vergleiche zu diesen U'mhüllenden-Eigenschaften beispielsweise Dixit und Norman (1982, 305-6). 
Um diesen Zusammenhang abzubilden, unterstellen wir streng monoton fallende, konvexe und differenzierbare Entsorgungskostenfunktionen $\mathrm{C}^{\mathrm{i}}$,

$$
c_{\mathrm{i}}=\mathrm{C}^{\mathrm{C}}\left(\mathrm{e}_{\mathrm{i}}\right) \quad(\mathrm{i}=1,2)
$$

mit der Eigenschaft $\mathrm{C}_{\mathrm{e}_{i}}\left(\mathrm{e}_{i}{ }^{0}\right)=0$ für ein $\mathrm{e}_{i}{ }^{0}>0$. Das aggregierte Emissionsniveau $e_{1}^{0}$ ist hierbei das Niveau, welches sich in einem rein auf dem privatwirtschaftlichen Entscheidungskalkül der Firmen eines Landes aufbauenden Marktgleichgewicht ohne staatliche Regulierung einstellen wird, d.h. ein Niveau, bei dem die Entsorgungskostenfunktionen ein Minimum annehmen.

Die zweite umweltpolitische Entscheidungsgrundlage der Regierung i bilden die in der Umweltregion i bei entsprechender Regulierungspolitik vermiedenen Schäden. Wir gehen davon aus, daß Regierung i einem Index der Immissionsbelastung $s_{i}$ einen bewerteten sozialen Schaden $d_{i}$ zuordnet, beispielsweise in Form von bewertbaren Produktionseinbußen, Vermögensschäden durch Beeinträchtigung des Konsums bereits hergestellter Güter oder Gesundheitskosten. ${ }^{10}$ Der Schaden steigt natürlicherweise mit zunehmender Immissionsbelastung. Auch diese Beziehung lasse sich durch monoton steigende, streng konvexe und differenzierbare Schadensfunktionen $\mathrm{D}^{\mathrm{i}}$,

$$
d_{1}=D^{i}\left(s_{1}\right)
$$$$
(i=1,2)
$$

mit der Eigenschaft $D^{i}\left(\underline{s}_{i}\right)=0$ für ein $\underline{s}_{i} \geq 0$ darstellen.

10) In der Literatur besteht Einmütigkeit darüber, daß eine direkte Feststellung eines bewerteten Schadens außer den beispielhaft genannten Formen unmöglich erscheint. Vergleiche dazu beispielsweise Siebert $(1976,39),(1978,69)$. Wenn eine nutzenma Bige Bewertung der Umweltqualität unmöglich erscheint, müssen die von Immissionen ausgelösten potentielle Schäden "politisch geschätzt" werden. Im Hinblick auf eine internationale Kooperation setzt das wiederum voraus, $\mathrm{da} \beta$ für alle Beteiligten einsichtige und akzeptierte Regeln bestehen, wie solch eine Bewertung vorzunehmen ist. Die Auswirkungen von asymmetrischer Information, die gerade in solchen Fällen bedeutsam sind, werden in einem der nachfolgenden Kapitel untersucht. 
Die Zielfunktionen der beiden Regierungen, die alle zur Bestimmung einer optimalen Umweltallokation im grenzüberschreitenden Umweltsystem relevanten Größen enthalten, werden durch die Summe von aggregierten Entsorgungskosten $\mathrm{C}^{1}$ bzw. $\mathrm{C}^{2}$ und Schäden $\mathrm{D}^{1}$ bzw. $\mathrm{D}^{2}$ gebildet. Die Summe von (3.2) und (3.3) sowie Einsetzen von (3.1) ergeben Umweltkostenfunktionen $\mathrm{K}^{1}$ und $\mathrm{K}^{2}$,

$$
\begin{aligned}
& K^{1}\left(e_{1}, e_{2}\right):=C^{1}\left(e_{1}\right)+D^{1}\left(a_{1}\left[\left(1-\beta_{1}\right) e_{1}+\beta_{2} e_{2}\right]\right), \\
& K^{2}\left(e_{1}, e_{2}\right):=C^{2}\left(e_{2}\right)+D^{2}\left(a_{2}\left[\beta_{1} e_{1}+\left(1-\beta_{2}\right) e_{2}\right]\right) .
\end{aligned}
$$

Diese Kostenfunktionen weisen unter den vorgegebenen Eigenschaften der Entsorgungskosten- und Schadensfunktionen bei partieller Variation des inländischen Emissionsniveaus einen u-förmigen Verlauf auf und haben bei einer entsprechenden Variation des ausländischen Emissionsniveaus einen konvex ansteigenden Verlauf. Mit Hilfe dieser Kostenfunktionen wird im nächsten Abschnitt die Frage beantwortet, zu welcher Umweltallokation diese Ausgangsbedingungen führen, wenn die beiden Regierungen ihre jeweiligen Regulierungspolitiken international nicht koordinieren.

\subsection{Die nichtkooperative Nash-Lösung bei unkoordinierter Umweltnutzung}

Wir gehen in diesem Abschnitt zunächst davon aus, daß die beiden Regierungen unkoordiniert - und damit jede für sich - im Sinne ihrer Zielsetzungen optimale Umweltnutzungen für die jeweiligen inländischen Umweltregionen bestimmen. Diese werden sofort und auf effiziente Art und Weise durch eine adäquate Regulierungspolitik durchgesetzt. Unter diesen Prämissen bleiben damit zunächst nur noch die beiden Fragen offen, welche Umweltallokation in einem möglichen Gleichgewicht erreicht wird und welche Effizienzeigenschaften dieses Gleichgewicht besitzt. 
Bei der Lösung dieser beiden Fragen muß beachtet werden, daß sich die beiden Länder durch das gemeinsame Umweltsystem in einer zueinander wechselseitigen Abhängigkeit befinden. Eine Veränderung der zulässigen Emissionen im Inland beeinflußt neben der inländischen auch die Umweltkostensituation des Auslands. Diese Beziehung besteht bis auf Randfälle, die sich als Oberlieger-Unterlieger-Verhältnisse charakterisieren lassen, immer gegenseitig. Unter diesen "Konkurrenzbedingungen" haben die Regulierungspolitiken der beiden Regierungen in den beiden Umweltregionen den Charakter eines nichtkooperativen 2-Personen-Nichtnullsummenspiels. ${ }^{11}$

\subsubsection{Das nichtkooperative Gleichgewicht}

Ausgehend von dem statischen Grundmodell, sind die in (3.4) definierten Umweltkostenfunktionen $\mathrm{K}^{1}$ und $\mathrm{K}^{2}$ die (negativen) Auszahlungsfunktionen des Spiels. Eine Strategie einer Regierung ist eine von ihr festgesetzte aggregierte Emissionsbeschränkung. Sie muß sinnvollerweise in dem abgeschlossenen Intervall liegen, das von der kleinstmöglichen Emission $e_{1}=0$ und der Emission $e_{i}=e_{i}{ }^{0}$, die die Firmen bei Nichtregulierung wählen würden, beschränkt wird. Die Strategiemenge $E^{i}$ einer Regierung $\mathrm{i}(\mathrm{i}=1,2)$ ist somit die Menge aller möglichen Emissionsbeschränkungen mit der Eigenschaft $E^{i}=\left[0, e_{i}^{0}\right] \subset \mathbf{R}_{+}$.

In dieser Situation mit nichtkooperativer Interaktion der beiden Regierungen unterstellen wir diesen ein individuell-rationales Verhalten, welches durch folgende vier Eigenschaften gekennzeichnet ist:

1. Eine Regierung i trifft ihre Entscheidung über die aggregierte Emissionsbeschränkung $e_{i}\left(e_{i} \in E^{i}\right)$ einzig und allein auf der Basis der in $\mathrm{E}^{1}, \mathrm{E}^{2}, K^{1}$ und $K^{2}$ enthaltenen Information.

11) Die Anwendung spieltheoretischer Konzepte bei der Lösung von Externalitätsproblemen geht auf Davis und Whinston (1962) zurück. Vergleiche dazu auch Campa (1967) und Shibata (1971). 
2. Bei der Wahl der eigenen Entscheidung geht eine Regierung davon aus, daß sich die andere Regierung genauso rational wie sie selbst verhält.

3. Wenn eine bestimmte Entscheidung für eine Regierung rational ist, dann ist diese Entscheidung von der anderen Regierung korrekt vorhersehbar.

4. Kann eine Regierung die Entscheidungen der andieren vorhersehen, dann minimiert sie ihre Umweltkosten entsprechend zu den vorhergesehenen Entscheidungen der anderen Regierung.

Johansen (1982) hat gezeigt, daß ein solches Verhalten ausschließlich kompatibel mit dem nichtkooperativen Nash-Gleichgewicht ist. In einem Gleichgewicht dieses Typs hat eine Regierung keinen Vorteil, wenn sie sich durch einseitige Wahl einer anderen als der Gleichgewichtsstrategie aus diesem herausbewegt. ${ }^{12)}$ Ein Strategiepaar $\left(\hat{\mathrm{e}}_{1}, \hat{\mathrm{e}}_{2}\right)$ ist demnach ein Nash-Gleichgewicht, wenn gilt

$$
\begin{array}{ll}
K^{\mathrm{i}}\left(e_{1}, \hat{\mathrm{e}}_{2}\right) \geq K^{1}\left(\hat{\mathrm{e}}_{1}, \hat{\mathrm{e}}_{2}\right) & \text { für alle } \mathrm{e}_{1} \in E^{1}, \\
K^{2}\left(\hat{\mathrm{e}}_{1}, \mathrm{e}_{2}\right) \geq K^{2}\left(\hat{\mathrm{e}}_{1}, \hat{\mathrm{e}}_{2}\right) & \text { für alle } e_{2} \in E^{2} .
\end{array}
$$

Das Paar $\left(\hat{\mathrm{e}}_{1}, \hat{\mathrm{e}}_{2}\right)$ beschreibt die aggregierten Emissionsbeschränkungen der beiden Regierungen in den Umweltregionen, die im Sinne der Verhaltensannahmen jeweils eine beste Reaktion einer Regierung auf das Verhalten der anderen darstellen.

Nash (1951) und in einem allgemeineren Modell ebenso Debreu (1952) haben die Existenz eines Gleichgewichts für ein Spiel dieses Typs nachgewiesen. Es genügt somit, das Gleichgewicht in unserem Fall durch dessen notwendige Bedingungen näher zu beschreiben.

12) Die Cournot-Nash-Reaktionshypothese ist daher eine sinnvolle Standardannahme in Modellen, die Probleme in Zusammenhang mit international öffentlichen Gütern behandeln. Vergleiche dazu Olson und Zeckhauser (1966), (1967), (1970), Williams (1966), Breton (1970), Pauly (1970), Conolly (1972), Sandler (1975), Kiesling (1976), Sandler und Culyer (1982a), (1982b). 
Die Differenzierbarkeit der Umweltkostenfunktionen erlaubt es, das Paar $\left(\hat{\mathrm{e}}_{1}, \hat{\mathrm{e}}_{2}\right)$ durch die notwendigen Bedingungen des Minimierungsproblems

$$
\operatorname{Min}_{e_{i} \in E^{i}} K^{i}\left(e_{i}, e_{h}\right) \quad(i, h=1,2, i \neq h)
$$

zu bestimmen. Für sie gilt

$$
K_{e_{i}}^{\prime}=C_{e}^{i}+D_{s}^{\prime} a_{i}\left(1-\beta_{1}\right)=0 \quad(i=1,2) .
$$

Dadurch werden implizit Reaktionsfunktionen $R^{i}\left(e_{h}\right)$ bestimmt. Die Wohldefiniertheit des Problems, Stetigkeit der Reaktionsfunktionen sowie die Kompaktheit und die Konvexität von $E^{1} \times E^{2}$, sichern, daß $\mathbf{R}^{\mathrm{i}} \circ \mathbf{R}^{\mathrm{h}}: \mathbf{R}_{+} \rightarrow \mathbf{R}_{+}$ mindestens einen Fixpunkt besitzt. Dieser bestimmt $\left(\hat{\mathrm{e}}_{1}, \hat{\mathrm{e}}_{2}\right)$ als Gleichgewichtslösung des Problems in (3.6). Diese Bedingung sichert aber noch nicht die Eindeutigkeit des Gleichgewichts, die somit noch zusätzlich untersucht werden muß.

Nach einem Satz von Friedman $(1977,165)$ ist ein solches Gleichgewicht eindeutig, wenn die Reaktionsfunktionen kontrahieren, d.h. wenn sich zeigen läßt, daß die Beziehung

$$
\left|\frac{\mathrm{de}_{\mathrm{i}}}{\mathrm{de}_{\mathrm{h}}}\right|<1 \quad(\mathrm{i}, \mathrm{h}=1,2, \mathrm{i} \neq \mathrm{h}) .
$$

gilt. Dazu differenziert man die notwendigen Bedingungen in (3.7) nach $\mathbf{e}_{1}$ sowie $e_{2}$ und erhält unter Berücksichtigung der unterstellten Krümmungseigenschaften von Entsorgungskosten- und Schadensfunktion, $\mathrm{C}^{\mathrm{i}}{ }_{\text {ep }} \geq 0$ sowie $\mathrm{D}_{\mathrm{ss}}^{\mathrm{i}}>0$,

$$
\frac{d e_{i}}{d e_{h}}=-\frac{D_{s s}^{i} a_{i}^{2}\left(1-\beta_{i}\right) \beta_{h}}{C_{e e}^{i}+D_{s s}^{i} a_{i}^{2}\left(1-\beta_{i}\right)^{2}} \leq 0 \quad(i, h=1,2, i \neq h) .
$$

Unter diesen Gegebenheiten kontrahieren die Reaktionsfunktionen, falls

$$
C_{e e}^{i}+D_{s s}^{i} a_{i}^{2}\left(1-\beta_{i}\right)^{2}>D_{s s}^{i} a_{i}^{2}\left(1-\beta_{i}\right) \beta_{h},
$$

bzw. 


$$
C_{e e}^{i}+D_{s s}^{i} a_{i}^{2}\left(1-\beta_{i}\right)\left(1-\beta_{1}-\beta_{2}\right)>0
$$

gilt. Diese Bedingung muß formal nicht notwendigerweise immer erfüllt sein. Sie kann verletzt werden, wenn $1-\beta_{1}-\beta_{2}$ negativ wird. Inhaltlich betrachtet können wir aber davon ausgehen, da $\beta$ die Verteilungskoeffizienten $\beta_{1}$ und $\beta_{2}$ nicht beide gleichzeitig einen hohen Wert annehmen, denn in diesem Fall wären beide Umweltregionen Oberlieger der jeweils anderen. Wir unterstellen daher im folgenden, daß die Bedingung (3.9) erfüllt und damit das Gleichgewicht eindeutig ist.

Graphisch läßt sich dieses Gleichgewicht durch den Schnittpunkt der beiden Reaktionsfunktionen in einer $\left(e_{1}, e_{2}\right)$-Ebene illustrieren. Beeinflussen sich die Emissionen beider Länder gegenseitig, d.h. $0<a_{i}<1$ und $0<\beta_{1}<1(i=1,2)$, dann weisen die Reaktionsfunktionen beider Länder

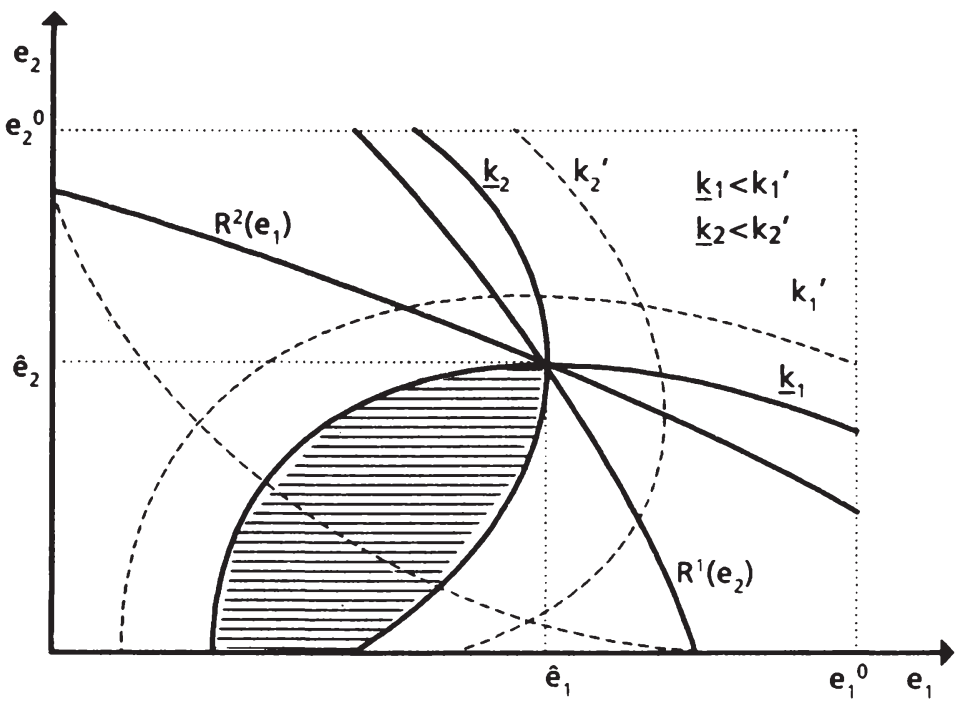

Abbildung 3.1 
gemäß (3.8) eine Steigung mit der Eigenschaft $-\infty<\partial R^{i} / \partial e_{h}<0$ auf. Abbildung 3.1 zeigt diesen Fall.

Die durch das Gleichgewicht verlaufenden Isokostenlinien $\underline{\mathbf{k}}_{1}$ und $\underline{\mathbf{k}}_{2}$,

$$
\begin{aligned}
& \underline{\mathbf{k}}_{1}:=\mathbf{K}^{1}\left(\hat{\mathbf{e}}_{1}, \hat{\mathbf{e}}_{2}\right), \\
& \underline{\mathbf{k}}_{2}:=\mathbf{K}^{2}\left(\hat{\mathbf{e}}_{1}, \hat{\mathbf{e}}_{2}\right),
\end{aligned}
$$

haben bei gegenseitiger Umweltbeeinflussung die Eigenschaft, daß sie an dieser Stelle senkrecht aufeinander stehen. Dies ist eine Konsequenz des Minimierungsansatzes in (3.6), da im jeweiligen Kostenminimum die Isokostenlinien relativ zu der Größe, über die optimiert wurde, eine Steigung von Null aufweisen. Zudem haben aufgrund der strengen Konvexität der Umweltkostenfunktionen die Isokostenlinien einen streng konkaven Verlauf. Isokostenlinien niedrigerer Umweltkostenniveaus liegen in Abbildung 3.1. unterhalb von $\underline{k}_{1}$ und links von $\underline{\mathbf{k}}_{2}$, da mit sinkendem ausländischen Emissionsniveau die inländischen Umweltkosten abnehmen. Somit existieren Paare $\left(e_{1}, e_{2}\right)$, die paretosuperior gegenüber dem Gleichgewichtspaar $\left(\hat{\mathrm{e}}_{1}, \hat{\mathrm{e}}_{2}\right)$ sind. In Abbildung 3.1 liegen diese paretosuperioren Emissionsbeschränkungen im Innern und auf dem Rand der durch die Isokostenlinien $\underline{\mathbf{k}}_{1}$ und $\underline{\underline{k}}_{2}$ gebildeten schraffierten Linse.

Die Ineffizienzeigenschaft dieses Gleichgewichts läßt sich analytisch dadurch zeigen, daß man die notwendigen Bedingungen in (3.7) mit den notwendigen Bedingungen für die Lösung des Pareto-Problems

$$
\begin{aligned}
& \operatorname{Min} K^{1}\left(e_{1}, e_{2}\right), \\
& e_{1}, e_{2} \\
& K^{2}\left(e_{1}, e_{2}\right) \leq k_{2}
\end{aligned}
$$

vergleicht. Die Lagrangefunktion L des Problems lautet

$$
L=-K^{1}\left(e_{1}, e_{2}\right)+\lambda\left[k_{2}-K^{2}\left(e_{i}, e_{2}\right)\right],
$$

wobei $\Lambda$ eine nichtnegative Lagrangevariable ist. Die notwendigen Bedingungen lauten: 


$$
\begin{aligned}
& L_{e_{i}}=-C^{1}{ }_{e}-D^{1}{ }_{s} a_{1}\left(1-\beta_{1}\right)-\lambda D^{2}, a_{2} \beta_{1}=0, \\
& L_{e_{2}}=-D^{1}{ }_{s} a_{1} \beta_{2}-\Lambda\left[C^{2}{ }_{e}+D^{2}{ }_{s} a_{2}\left(1-\beta_{2}\right)\right]=0 .
\end{aligned}
$$

Aufgrund der Konkavität des Problems sind diese Bedingungen gleichzeitig hinreichend. Sie lassen sich umformen zu

$$
\begin{aligned}
& -C_{e}^{1}-D_{s}^{1} a_{1}\left(1-\beta_{1}\right)=\lambda D_{s}^{2} a_{2} \beta_{1} . \\
& -C_{e}^{2}-D_{s}^{2} a_{2}\left(1-\beta_{2}\right)=\frac{1}{\lambda} D_{s}^{1} a_{1} \beta_{2},
\end{aligned}
$$

Die rechten Seiten der Gleichungen stellen den Schattenpreis der ausländischen Umweltnutzung durch das Inland dar. Solange im Optimum die Nebenbedingung bindend ist und damit $\lambda>0$, sind bei gegenseitiger Umweltbeeinflussung, d.h. strikt positiven Koeffizienten $a_{1}, a_{2}, \beta_{1}$ und $\beta_{2}$, diese Schattenpreise strikt positiv. Aufgrund des u-förmigen Verlaufs der Umweltkostenfunktionen in Bezug auf das inländische Emissionsniveau und in Verbindung mit den notwendigen Bedingungen für das unkoordinierte Gleichgewicht in (3.7), existieren negative Grenzumweltkosten nur für $e_{1}<\hat{e}_{1}$ bzw. $e_{2}<\hat{e}_{2}$. Folglich müssen die paretooptimalen Emissionsbeschränkungen $\left(e_{1}^{\prime}, e_{2}^{\prime}\right)$ beide strikt kleiner als die Emissionsbeschränkungen $\left(\hat{\mathrm{e}}_{1}, \hat{\mathrm{e}}_{2}\right)$ des Gleichgewichts bei unkoordinierter Umweltnutzung sein, d.h.

$$
\left(\mathbf{e}_{1}^{\prime}, \mathbf{e}_{2}{ }^{\prime}\right)<\left(\hat{\mathbf{e}}_{1}, \hat{\mathbf{e}}_{2}\right)
$$

In Abbildung 3.1 liegen die geometrischen Orte der paretooptimalen Emissionsbeschränkungen als Tangentialpunkte von Isokostenlinien unterhalb der beiden Reaktionsfunktionen.

Führen steigende Assimilationskräfte in einer Umweltregion oder einseitige Verteilungskräfte dazu, daß mindestens einer der Koeffizienten $a_{1}$, $a_{2}, \beta_{1}$ und $\beta_{2}$, den Wert Null annimmt, dann verbessern sich die Effizienzeigenschaften des unkoordinierten Gleichgewichts $\left(\hat{\mathrm{e}}_{1}, \hat{\mathrm{e}}_{2}\right)$. In diesem Fall 
stimmt mindestens eine der Bedingungen (3.11) und (3.12) mit der entsprechenden Bedingung für das unkoordinierte Gleichgewicht in (3.7) überein. Ein Pareto-Optimum muß demzufolge auf einer der Reaktionslinien liegen. Die nachfolgende komparativ-statische Analyse dient insbesondere dazu, den Einfluß spezieller Eigenschaften des Umweltsystems auf die Gleichgewichtsallokation klarer herauszuarbeiten.

\subsubsection{Die Auswirkungen von Parameteränderungen auf das Gleichgewicht}

Es gibt eine Vielzahl exogener Einflußgrößen, die die Gleichgewichtsallokation im Umweltsystem und damit die Höhe und Verteilung der Umweltkosten zwischen den beiden Ländern determinieren. Diese lassen sich zwei Teilbereichen zuordnen. Der erste Teilbereich betrifft die Einflußgrößen der Produktions- und Konsumbedingungen in den beiden Umweltregionen. Den zweiten Teilbereich bilden die exogenen Größen, die die spezifischen Eigenheiten des grenzüberschreitenden Umweltsystems bestimmen.

Der erste Teilbereich schließt somit alle Parameter ein, die die Höhe der Entsorgungskosten und der Schäden verändern. Dazu gehören beispielsweise Standortüberlegungen von emittierenden Firmen oder der Geschädigten. ${ }^{13)}$ In diesem Zusammenhang sind die potentiellen Entsorgungskosten umso geringer, je kleiner der Anteil emissionsintensiver Produktion in einer Umweltregion ist. Ähnliches gilt für die durch Immissionen verursachten Schäden, die um so geringer sind, je kleiner die Gruppe der Konsumenten und Produzenten ist, die von den spezifischen Immissionswirkungen in diesem Umweltsystem betroffen wird. Als zweites Beispiel

13) Tiebout (1956) betrachtet solche Überlegungen als ein endogenes Problem, wonach Konsumenten ihre Umweltpräferenzen durch ihre Wohnortentscheidung (voting with one's feet) offenbaren. Der Teil der Umweltliteratur, der sich mit regionalen Aspekten des Umweltproblems auseinandersetzt, hat das Tiebout-Theorem weitgehend abgelehnt, da es nur unter sehr restriktiven Annahmen gültig ist. Vergleiche dazu auch Dudenhöffer (1983). 
läßt sich der entsorgungskosten- bzw. schadensenkende technische Fortschritt anführen, der Produktionstechniken hinsichtlich des Anteils der schadstoffhaltigen Kuppelprodukte oder auch Entsorgungs- und Aufbereitungstechnologien verbessert.

Den Einfluß exogener Größen, die die Entsorgungskostenfunktionen $\mathrm{C}^{i}$ und Schadensfunktionen $D^{i}$ verändern, wollen wir kurz skizzieren und dabei von einer Situation mit gegenseitiger Umweltbeeinflussung ausgehen. In diesem Fall würde eine durch exogene Größen ausgelöste Entsorgungskosten- bzw. Schadensänderung im Inland zu Rückkopplungseffekten aus dem Ausland führen.

Beispielsweise impliziert eine Senkung der Grenzkosten der Entsorgung gemäß (3.7) bei gegebenem Grenzschaden und gegebenem ausländischen Emissionsniveau eine Reduktion der zulässigen inländischen Emissionen. Dadurch sinkt der Schaden in der ausländischen Umweltregion. Das Ausland reagiert darauf durch ein Heraufsetzen der eigenen Emissionsgrenze. Das hat zur Folge, daß die durch den inländischen Entsorgungskostenvorteil verringerten inländischen Entsorgungskosten und Schäden wieder teilweise steigen.

Eine sehr ähnliche Wirkungskette würde bei einem Anstieg des inländischen Grenzschadens ausgelöst. Solche durch exogene Veränderungen der inländischen Umgebungsbedingungen ausgelösten Rückkopplungseffekte führen somit zu einer Umverteilung der Umweltkosten zwischen den Ländern. Ein Kostenvorteil des Inlands wird durch die Reaktion des Auslands wieder verringert.

Entscheidend für den Einfluß des Umweltsystems auf die Gleichgewichtsallokation sind dessen Assimilations- und Verteilungskräfte. Um diesen Einfluß zu messen, differenzieren wir total die notwendigen Bedingungen in (3.7). Dabei erhalten wir folgendes Gleichungssystem: 


$$
\begin{aligned}
& {\left[\begin{array}{ll}
C_{e e}^{1}+D_{s s}^{1} a_{1}{ }^{2}\left(1-\beta_{1}\right)^{2} & D^{1}{ }_{s s} a_{1}{ }^{2}\left(1-\beta_{1}\right) \beta_{2} \\
D_{s s}^{2} a_{2}{ }^{2} \beta_{1}\left(1-\beta_{2}\right) & C^{2}{ }_{e e}+D^{2}{ }_{s s} a_{2}{ }^{2}\left(1-\beta_{2}\right)^{2}
\end{array}\right]\left[\begin{array}{l}
\operatorname{de}_{1} \\
d_{2}
\end{array}\right]} \\
& =\left[\begin{array}{cc}
-\left(D_{s s}^{1} s_{1}+D_{s}^{1}\right)\left(1-\beta_{1}\right) & 0 \\
0 & -\left(D^{2}{ }_{s s} s_{2}+D^{2}{ }_{s}\right)\left(1-\beta_{2}\right)
\end{array}\right]\left[\begin{array}{l}
\mathrm{da}_{1} \\
\mathrm{da}_{2}
\end{array}\right] \\
& +\left[\begin{array}{ll}
{\left[D_{s s}^{i} a_{1}\left(1-\beta_{1}\right) e_{1}+D^{1}{ }_{s}\right] a_{1}} & -D^{i}{ }_{s s} a_{1}{ }^{2}\left(1-\beta_{1}\right) e_{2} \\
-D_{s s}^{2} a_{2}^{2}\left(1-\beta_{2}\right) e_{1} & {\left[D^{2}{ }_{s s} a_{2}\left(1-\beta_{2}\right) e_{2}+D^{2}{ }_{s}\right] a_{2}}
\end{array}\right]\left[\begin{array}{l}
d \beta_{1} \\
d \beta_{2}
\end{array}\right]
\end{aligned}
$$

Für dessen Systemdeterminante $\Delta_{1}$ gilt

$$
\begin{aligned}
& \Delta_{1}=\left[C^{1}{ }_{e e}+D_{s s}^{1} a_{1}{ }^{2}\left(1-\beta_{1}\right)^{2}\right]\left[C^{2}{ }_{e e}+D_{s s}{ }_{s i} a_{2}{ }^{2}\left(1-\beta_{2}\right)^{2}\right] \\
& -D_{s s}^{i} a_{1}^{2}\left(1-\beta_{1}\right) \beta_{2} D_{s s}^{i} a_{2}^{2} \beta_{1}\left(1-\beta_{2}\right)>0 \text {. }
\end{aligned}
$$

Verringern sich die Assimilationskräfte, dann steigen die Koeffizienten $a_{1}$ und/oder $a_{2}$. Die damit verbundenen Wirkungen auf die Emissionsbeschränkungen des Gleichgewichts lassen sich mit Hilfe der Cramerschen Regel aus diesem Gleichungssystem bestimmen. Es gilt

$$
\frac{\mathrm{de}_{1}}{\mathrm{da} a_{1}}=\frac{-\left[D_{s s}^{1} s_{1}+D_{s}^{1}\right]\left(1-\beta_{1}\right)\left|C_{e e}^{2}+D_{s s}^{2} a_{2}^{2}\left(1-\beta_{2}\right)\right|}{\Delta_{1}}<0,
$$




$$
\frac{d e_{2}}{d a_{1}}=\frac{\left|D_{s s}^{1} s_{1}+D_{s}^{1}\right|\left(1-\beta_{1}\right)\left|D_{s s}^{2} a_{2}^{2} \beta_{1}\left(1-\beta_{2}\right)^{2}\right|}{\Delta_{1}}>0 .
$$

Analog dazu läßt sich aufgrund der Symmetrie des Gleichungssystems durch Vertauschen der Indizes $\mathrm{de}_{1} / \mathrm{da}_{2}>0$ und $\mathrm{de}_{2} / \mathrm{da}_{2}<0$ ermitteln. Verringern sich die Assimilationskräfte im Inland, dann wirkt dieses wie eine Erhöhung des inländischen Grenzschadens und führt dazu, daß das inländische Emissionsniveau gesenkt und das ausländische erhöht wird. ${ }^{14)}$

Entsprechendes ergibt sich, wenn die Verteilungskräfte steigen, d.h. der Koeffizient $\beta_{1}$ oder (plausiblerweise) $\beta_{2}$ sich erhöht. In diesem Fall gilt für die Emissionsbeschränkungen im Gleichgewicht

$$
\begin{aligned}
& \frac{d e_{1}}{d \beta_{1}}=\frac{\left|D_{s s}^{1} a_{1}\left(1-\beta_{1}\right) e_{1}+D_{s}^{1}\right| a_{1}\left|C_{e e}^{2}+D_{s s}^{2} a_{2}^{2}\left(1-\beta_{2}\right)^{2}\right|+D_{s s}^{2} a_{2}^{2} e_{1}\left(1-\beta_{2}\right) D_{s s}^{1} a_{1}^{2} \beta_{2}\left(1-\beta_{1}\right)}{\Delta_{1}}>0, \\
& \frac{d e_{2}}{d \beta_{1}}=\frac{-\left|D_{s s}^{1} a_{1}\left(1-\beta_{1}\right) e_{1}+D_{s}^{1}\right| a_{1} D_{s s}^{2} a_{2}^{2} \beta_{1}\left(1-\beta_{2}\right)-D_{s s}^{2} a_{2}^{2}\left(1-\beta_{2}\right) e_{1}\left|C_{e e}^{1}+D_{s s}^{1} a_{1}^{2}\left(1-\beta_{1}\right)^{2}\right|}{\Delta_{1}}<0,
\end{aligned}
$$

sowie wiederum aufgrund der Symmetrie de $e_{1} / d \beta_{2}<0$ und $d e_{2} / d \beta_{2}>0$. Eine verstärkte Verteilungswirkung des Umweltsystems vom Inland ins Ausland bedeutet faktisch eine Senkung des inländischen Grenzschadens, aber gleichzeitig eine Erhöhung des ausländischen Grenzschadens. Infolgedessen werden die inländischen Emissionsbeschränkungen gelockert und die ausländischen verschärft. ${ }^{151}$

14) Graphisch betrachtet, entspricht dies einer Verschiebung des Gleichgewichtspunkts auf der Reaktionsfunktion des Auslands in Richtung auf ihren "Monopolpunkt", d.h. den Punkt auf der Emissions-Achse, den die ausländische Regierung bei einer inländischen Emission von Null wählen würde

15) In Abbildung 3.1 entspricht dies einer Drehung der ausländischen Reaktionsfunktion im Monopolpunkt hin zum Lirsprung und einer Verschiebung der inlăndischen Reaktionsfunktion weg vom Lisprung 
Die in diesen Ableitungen wieder sichtbaren Rückkopplungseffekte werden unterbunden, wenn aufgrund der spezifischen Eigenheiten des Umweltsystems eine Verteilung der Emissionen vorwiegend in eine Richtung erfolgt. Ein solches System mit Oberlieger-Unterlieger-Eigenschaften stellt sich auch hinsichtlich der Effizienzeigenschaften des Gleichgewichts als ein Sonderfall dar.

Das Inland wird zum faktischen Oberlieger des Auslands, wenn Emissionen des Auslands in der inländischen Umweltregion keinerlei Schaden anrichten. Im Rahmen unseres Modells ist das zum einen der Fall, wenn der ausländische Verteilungskoeffizient sehr klein ist, d.h. wenn nur sehr geringe Schadstoffmengen vom Ausland ins Inland "importiert" werden. Diese Situation besteht zum anderen auch dann, wenn der inländische

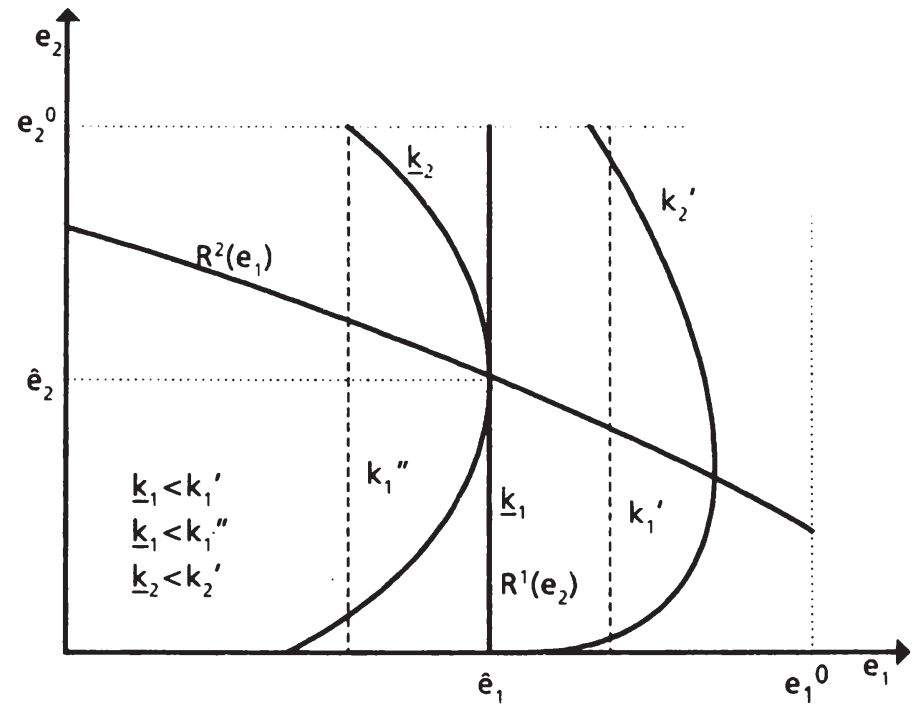

Abbildung 3.2 
Assimilationskoeffizient sehr klein ist, d.h. wenn die Assimilationskräfte in der inländischen Umweltregion so hoch sind, $\mathrm{da} B$ dort keinerlei Schäden angerichtet werden können. In solchen Situationen weist im Grenzfall die Reaktionsfunktion des Oberliegers die Steigung Null auf. Abbildung 3.2 zeigt dies für den Fall, in dem die Umweltregion 1 Oberlieger der Umweltregion 2 ist, d.h. entweder für den Verteilungskoeffizienten $\beta_{2}=0$ und/oder für den Assimilationskoeffizenten von Umweltregion $1 a_{1}=0$ gilt.

Aufgrund der Unabhängigkeit der Umweltkosten der Umweltregion 1 vom Emissionsniveau $e_{2}$ ist in diesem Fall die Reaktionsfunktion $R^{1}\left(e_{2}\right)$ von Regierung 1 identisch mit der Isokostenlinie $\underline{k}_{1}$ für die minimalen Umweltkosten. Infolge des u-förmigen Verlaufs der Umweltkostenfunktion im inländischen Emissionsniveau liegen rechts und links davon Isokostenlinien für höhere Kostenniveaus.

Bei diesem Extremfall ist das unkoordinierte Gleichgewicht $\left(\hat{\mathrm{e}}_{1}, \dot{\mathrm{e}}_{2}\right)$ paretooptimal. Die notwendige Bedingung für Pareto-Optimalität in (3.12) stimmt mit der entsprechenden Bedingung für das unkoordinierte Gleichgewicht in (3.7) überein. Folglich bilden alle Punkte auf der Reaktionslinie des Unterliegers zwischen ihrem Ursprung an der $\mathrm{e}_{2}$-Achse für die Unterlieger-Emissionen und dem Gleichgewichtspunkt $\left(\hat{\mathrm{e}}_{1}, \hat{\mathrm{e}}_{2}\right)$ die geometrischen Orte aller paretooptimalen Umweltallokationen in diesem Grenzfall der Ökonomie.

\subsection{Zusammenfassung}

Faßt man die bisherigen Ergebnisse zusammen, so wurde gezeigt, daß eine international unkoordinierte Regulierungspolitik der beiden Länderregierungen unserer Modellökonomie bis auf reine Oberlieger-UnterliegerSituationen zu einer ineffizienten Allokation der Umweltressource im gemeinsamen Umweltsystem führt. Daraus leitet sich die Frage ab, ob 
eine internationale Koordination der Regulierungspolitiken eine Verbesserung der Allokation bewirken kann.

Bei einem Umweltsystem mit gegenseitiger Beeinflussung erscheint eine koordinierte Umweltallokation gegenüber einer unkoordinierten immer vorteilhaft. Geht man beispielsweise vom Gleichgewichtspunkt $\left(\hat{\mathbf{e}}_{1}, \hat{\mathbf{e}}_{2}\right)$ als der Vergleichssituation bei unkoordinierter Umweltnutzung aus, existieren nach der Gleichgewichtsanalyse paretosuperiore $\left(e_{1}, e_{2}\right)$-Kombinationen, die diesen streng dominieren. Die Oberlieger-Unterlieger-Situation unterscheidet sich möglicherweise davon. Unterstellt man das in diesem Fall paretooptimale Gleichgewicht wieder als Ausgangspunkt, sind Verhandlungen nur dann erfolgversprechend, wenn der Oberlieger bei den Entsorgungskosten einen absoluten Vorteil gegenüber den Kosten hat, die dem Unterlieger bei der Schadensbeseitigung entstehen. Dieser Vorteil kann unter dem Postulat eines individuell-rationalen Verhaltens nur durch eine Ausgleichszahlung bzw. Entsorgungskostenbeteiligung realisiert werden, mit der der Unterlieger dem Oberlieger eine entsprechende Verschärfung der Emissionsbeschränkung abkauft.

Das Koordinationsproblem werden wir in den nächsten Kapiteln näher untersuchen, zunächst in einem sehr einfachen statisch-axiomatischen Ansatz unter "idealen" Rahmenbedingungen ohne potentielle Transaktionskosten, wie ihn auch Pethig (1982) in einem ähnlichen Modell verwandt hat. In den darauffolgenden Kapiteln erweitern wir diesen Ansatz zu einem dynamisch-strategischen Modell. Dieser Schritt bietet die Möglichkeit, spezielle Formen potentieller Transaktionskosten zu analysieren, die sich aus den Rahmenbedingungen des Koordinationsprozesses ergeben. Dazu zählen beispielsweise der Einfluß der zeitlichen Organisation des Verhandlungsprozesses, zwischenzeitliche Veränderungen der Ausgangsbedingungen im Umweltsystem und der Einfluß von Informationsunvollkommenheiten. 


\section{Die statisch-axiomatische Lōsung bei koordinierter Umweltnutzung}

Im vorangegangenen Kapitel wurde deutlich, daß mit einer internationalen Koordination der Regulierungspolitiken eine paretosuperiore Allokation der gemeinsamen Umweltressource erreicht werden kann. Das trifft auch auf den im unkoordinierten Gleichgewicht, gemessen an den Möglichkeiten in dieser Situation, effizienten Oberlieger-Unterlieger-Fall zu, wenn mit Hilfe von Kompensationszahlungen Kostenvorteile zwischen Oberlieger und Unterlieger realisiert werden können. $\mathrm{Zu}$ diesem $\mathrm{Z}$ weck treten die Regierungen der beiden Länder unserer Modellökonomie in Verhandlungen. Diese zielen darauf ab, eine gemeinsame institutionelle Regelung zu vereinbaren, worin sich die Regierungen verpflichten, den entsprechenden nationalen Umweltressourceneinsatz zu beschränken.

Gegenstand dieses Kapitels ist, die Verhandlungssituation unter "idealen" Bedingungen zu beschreiben. Im Sinne des "Coase-Theorems" gehen wir von einem vollkommenen Verhandlungsmarkt aus, auf dem Verhandeln für beide Regierungen mit keinerlei Transaktionskosten verbunden ist. Das schließt ein, daß sich diese nur auf solche Lösungen einigen, bei denen keine von beiden schlechter als in der Ausgangssituation gestellt ist. Es wird somit zunächst von der realistischeren Vorstellung abstrahiert, nach der Verhandlungsmärkte unvollkommen sind und sich Verhandeln als kostenintensiver Prozeß darstellt.

Zur spieltheoretischen Modellierung des Verhandlungsproblems unterstellen wir einen statisch-axiomatischen Ansatz, der auf dem kooperativen Nash-Verhandlungsmodell aufbaut. ${ }^{1}$ Die für diesen Ansatz charakteristischen Axiome stellen Annahmen über die Eigenschaften und damit die Zulässigkeit bestimmter Verhandlungsergebnisse dar. Dadurch vereinfacht sich die Darstellung des Gesamtproblems, weil der eigentliche Verhandlungsproze $\beta$ nicht mehr beschrieben werden muß.

1) Dieser Ansatz gehört zur sogenannten Teilungstheorie, die auf Zeuthen (1930), Nash (1950) und Harsanyi (1956) zurückgeht. Einen zusammenfassenden geben u.a. Krelle $(1976,598 \mathrm{ff}$.) und Harsanyi $(1977,141 \mathrm{ff}$.). 
Der Fall gegenseitiger Umweltbeeinflussung und damit die CommonPool-Problematik von Umweltressourcen und der Oberlieger-UnterliegerFall werden nicht länger getrennt dargestellt. Beide Fälle lassen sich gemeinsam in einem Verhandlungsmodell analysieren, da letzterer ein Spezialfall des ersteren ist. Die für eine Einigung im Oberlieger-Unterlieger-Fall notwendigen Ausgleichszahlungen verallgemeinern zudem das Modell. Will man spezielle Verhandlungssituationen betrachten, in denen Ausgleichszahlungen nicht zugelassen sind, läßt sich diese Erweiterung modelltheoretisch entsprechend wieder einschränken.

Abschnitt 4.1. beschreibt das statische Verhandlungsmodell auf der Basis des im vorausgegangenen Kapitels dargestellten Grundmodells einer 2Länder-Ökonomie und bestimmt mögliche effiziente Verhandlungsergebnisse und ihre Veränderungen unter alternativen Ausgangsbedingungen im Umweltsystem. Danach werden in Abschnitt 4.2. mit Hilfe eines erweiterten kooperativen Nash-Verhandlungsmodells mögliche Verhandlungsgleichgewichte bestimmt. Abschnitt 4.3. nimmt kritisch Stellung zu diesem Gleichgewichtskonzept und Abschnitt 4.4. faßt die Ergebnisse dieses Kapitels abschließend zusammen.

\subsection{Das statische Verhandlungsmodell}

Formal wird eine Verhandlungssituation im allgemeinen wie folgt beschrieben: Erstens durch den Gegenstand der Verhandlungen und damit durch die Form von Verhandlungsangeboten, zweitens durch das Regelwerk wie der eigentliche Verhandlungsprozeß abläuft und eine Einigung zustandekommt und drittens durch die Präferenzen der Verhandelnden über mögliche Verhandlungsergebnisse. Das statische Verhandlungsmodell vereinfacht diese Zusammenhänge, indem es sich auf die Verhandlungsergebnisse konzentriert und von dem eigentlichen Verhandlungsprozeß weitgehend abstrahiert. 


\subsubsection{Umweltkostenvorteile}

Der Verhandlungsgegenstand der beiden Regierungen in unserer Modellökonomie besteht darin, gemeinsam zu bestimmen, wie eine für beide Länder vorteilhaftere, koordinierte Umweltallokation in dem grenzüberschreitenden Umweltsystem beschaffen sein sollte. Dazu ist es notwendig, daß die Regierungen eine einstimmige Entscheidung über die möglichen Koordinierungsalternativen treffen müssen.

Im einfachsten Fall, von dem wir hier ausgehen, besteht eine solche Alternative aus einem Vorschlag für die Emissionsbeschränkungen $e_{1}$ und $e_{2}$ in beiden Umweltregionen sowie einem Vorschlag für Ausgleichszahlungen $z$, die netto zwischen den beiden Ländern transferiert werden sollen. Eine solche von einer Regierung vorgeschlagene Alternative $\mathrm{a}=\left(\mathrm{e}_{1}, \mathrm{e}_{2}, \mathrm{z}\right)$ bezeichnen wir als Angebot einer Regierung. Darin spezifiziert neben den Emissionsbeschränkungen $e_{1}$ und $e_{2}$ die Größe z die Höhe der Nettoausgleichszahlungen, die von Land 2 an Land $1 \mathrm{bzw}$. von Umweltregion 2 an Umweltregion 1 geleistet werden. Es seien $A^{1}$ und $A^{2}$ die Mengen, die jeweils alle die Angebote enthalten, die Regierung 1 bzw. $2 \mathrm{im}$ Verlauf des Verhandlungsprozesses abgeben würde. Im folgenden wird noch genauer spezifiziert werden, welchen Eigenschaften diese Angebotsmengen $A^{1}$ und $A^{2}$ genügen. Ein möglicher Verhandlungsproze $B$ wird durch Folgen solcher Verhandlungsangebote beschrieben und zudem Regeln, die festlegen, in welchen Fällen eine Einigung zustande kommt und in welchen Fällen nicht. ${ }^{2 \prime}$

2) In der Theorie werden diese Abläufe durch die Vorgabe einer regelmäßigen Struktur sehr stark vereinfacht. Es finden sich im wesentlichen zwei verschiedene Szenarien. In einem von ihnen wählen die Verhandlungsparteien simultan Verhandlungsangebote aus den jeweiligen Angebotsmengen aus. Sind die ausgewählten Angebote im Sinne der individuellen Präferenzen kompatibel miteinander, kommt eine vertragliche Vereinbarung zustande. Sind sie inkompatibel, wird der Auswahlprozeß erfolglos abgebrochen. In dem anderen Szenario gibt entweder nur eine Partei oder es geben beide abwechselnd sukzessiv Angebote ab, auf die jeweils die nichtanbietende Partei durch Annahme oder Ablehnung reagieren muß. Nimmt diese an, gilt die Vereinbarung als zustande gekommen. Lehnt sie dagegen ab, beginnt der Auswahlprozeß durch sie oder die andere Partei von neuem. Er wird solange fortgesetzt bis eine Vereinbarung erreicht oder durch die Verhandlungsregeln ein Abbruch, beispielweise durch eine Beschränkung der Verhandlungsrunden, erzwungen wird. 
Das statische Verhandlungsmodell abstrahiert von der dynamischen Darstellung eines Verhandlungs- und Einigungsprozesses. Es geht vereinfachend davon aus, daß solche, diesen Prozeß beschreibende Folgen von Verhandlungsangeboten aus den Mengen $A^{1}$ und $A^{2}$ existieren und zu einem bestimmten Ergebnis führen. Umgekehrt argumentiert man aber auch, daß jedes mögliche Ergebnis durch die Wahl entsprechender Angebotsfolgen erreichbar ist. Damit macht man sich zunächst vollkommen unabhängig davon, wie der Verhandlungsproze $\beta$ tatsächlich abläuft, bzw. ob er irgendwelchen Restriktionen unterliegt.

Gegenstand der Betrachtung im statischen Verhandlungsmodell sind nur die möglichen Verhandlungsergebnisse. Das sind in unserem Fall zunächst die Umweltkostenvorteile, die die Regierungen 1 und 2 bei bestimmten Emissionsbeschränkungen $e_{1}, e_{2}$ und bestimmten Ausgleichszahlungen $z$ gegenüber den Kosten einer Referenzsituation genießen.

Wenn wie in Kapitel 3 von einer aus rechtlicher Sicht internationalen laissez-faire-Situation ausgegangen wird, sind die adäquaten Referenzkosten die minimalen Umweltkosten, die die Regierungen ohne Verhandlungen erreichen können, d.h. die Kostenniveaus $\underline{k}_{1}=K^{1}\left(\hat{e}_{1}, \hat{e}_{2}\right)$ und $\underline{\mathbf{k}}_{2}=\mathrm{K}^{2}\left(\hat{\mathrm{e}}_{1}, \hat{\mathrm{e}}_{2}\right)$ im Gleichgewicht bei unkoordinierter Umweltnutzung. Diese haben die Funktion einer besten Alternative für den Fall, daß Verhandlungen ergebnislos enden. Bei internationalen haftungs- und völkerrechtlichen Einschränkungen der Verfügungsrechte an der Ressource oder Beschränkungen, die durch eine einheitliche Umweltpolitik für Umweltsysteme dieser Art in einem Land bestehen, sind natürlich andere Referenzkosten maßgebend. Im folgenden sei aber zunächst von der laissez-faire-Situation und damit Referenzkosten $\left(\underline{\mathbf{k}}_{1}, \underline{\mathbf{k}}_{2}\right)$ ausgegangen, die wir als Status quo bezeichnen.

Die bei einem Angebot $a=\left(e_{1}, e_{2}, z\right)$ von den Regierungen 1 und 2 erreichbaren Umweltkostenvorteile $\pi_{1}$ bzw. $\pi_{2}$ werden durch die Funktionen $\Pi^{1}$ und $\Pi^{2}$, 


$$
\begin{aligned}
& \Pi^{1}\left(\mathbf{e}_{1}, \mathbf{e}_{2}, \mathbf{z}\right):=\underline{\mathbf{k}}_{1}-K^{1}\left(\mathbf{e}_{1}, \mathbf{e}_{2}\right)+\mathbf{z}, \\
& \Pi^{2}\left(\mathbf{e}_{1}, \mathbf{e}_{2}, \mathbf{z}\right):=\underline{\mathbf{k}}_{2}-K^{\prime \prime}\left(\mathbf{e}_{1}, \mathbf{e}_{2}\right)-\mathbf{z},
\end{aligned}
$$

gemessen. Ein solcher Umweltkostenvorteil einer Umweltregion läßt sich auch als ein Verhandlungsgewinn interpretieren.

Die Summe aus den beiden Umweltkostenvorteilen beschreibt den in der Ökonomie bei einer koordinierten Variation der Emissionsbeschränkungen $\left(e_{1}, e_{2}\right)$ gegenüber dem Status quo $\left(\hat{e}_{1}, \hat{e}_{2}\right)$ erreichbaren Effizienzgewinn $\Pi$,

$$
\begin{aligned}
\Pi\left(e_{1}, e_{2}\right): & \equiv \Pi^{1}\left(e_{1}, e_{2}, z\right)+\Pi^{2}\left(e_{1}, e_{2}, z\right) \\
& \equiv \underline{k}_{1}+\underline{k}_{2}-K^{1}\left(e_{1}, e_{2}\right)-K^{2}\left(e_{1}, e_{2}\right) .
\end{aligned}
$$

Dieser ist natürlich unabhängig von der über die Ausgleichszahlung z möglichen Umverteilung zwischen den Ländern.

Entsprechend dem unterstellten Rationalitätspostulat für das Verhalten von Regierungen in Verhandlungen, werden Regierung 1 und 2 nur solche Angebote abgeben und auch akzeptieren, die die Umweltregion 1 bzw. 2 gegenüber dem Status quo $\underline{\mathbf{k}}_{1}$ bzw. $\underline{\mathbf{k}}_{2}$ mindestens so gut oder besser stellen. Die Mengen $A^{1}$ und $A^{2}$ aller im klassischen Sinne individuellrationalen ${ }^{3)}$ Angebote werden somit durch

$$
A^{\prime}:=\left\{a=\left(e_{1}, e_{2}, z\right) \mid \Pi^{1}\left(e_{1}, e_{2}, z\right) \geq 0\left(e_{1} \in E^{1}, e_{2} \in E^{2}, z \in R\right)\right\}, \quad i=1,2
$$

definiert. Zu einer Einigung kann es aber nur über solche Angebote kommen, bei denen beide Regierungen einen Umweltkostenvorteil erzielen. Diese für spätere Gleichgewichte allein relevanten Angebote

3) Gemäß der Definition in der klassischen Entscheidungstheorie beschreibt der Begriff "individuell-rational" die Eigenschaft, daßs eine Vereinbarung zwischen beiden Regierungen für jede von ihnen mindestens so gut oder besser wie eine Vergleichssituation sein muß, d.h. $\Pi^{\prime} \geq 0$. Vergleiche dazu auch Harsanyi (1977, 141-2). Johansen (1982) versteht diesen Begriff in Hinblick auf nichtkooperative Spiele umfassender. Vergleiche dazu auch Abschnitt 3.2.1. 
beschreibt vollständig die aus den Angebotsmengen $A^{1}$ und $A^{2}$ gebildete Schnittmenge $A$,

$$
A:=A^{1} \cap A^{2} .
$$

Durch die Menge $A$ wird gleichzeitig die Menge $\Pi$ aller bei Verhandlungen für beide Regierungen potentiell einigungsfähigen Umweltkostenvorteile definiert,

$$
\Pi:=\left\{\left(\Pi^{1}(a), \Pi^{2}(a)\right) \mid a \in A\right\} .
$$

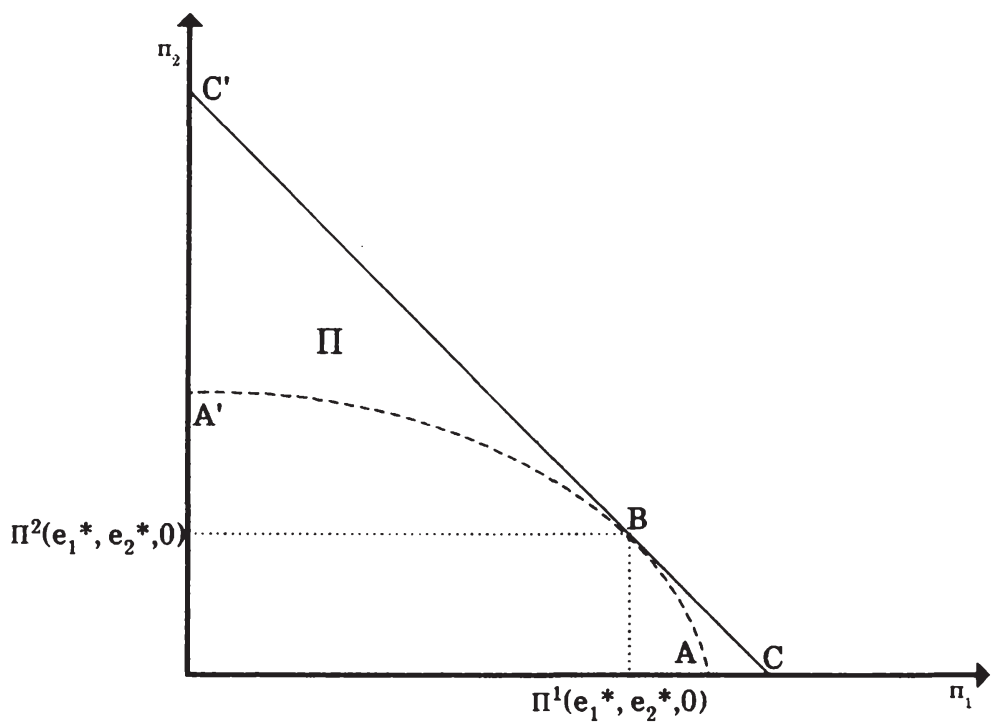

Abbildung 4.1 
In Abbildung 4.1 werden diese für einen typischen Fall mit gegenseitiger Umweltbeeinflussung graphisch dargestellt, in Abbildung 4.2 für einen Oberlieger-Unterlieger-Grenzfall. Dabei entspricht der Menge $\Pi$ die gesamte Fläche $O C C$ ' in beiden Abbildungen.

Dadurch, daß in Abbildung 4.1 die Umweltkosten der Status-quoSituation gleich den Umweltkosten im Gleichgewicht bei unkoordinierter Umweltallokation sind, kann man diese direkt mit der Abbildung 3.1 vergleichen. Die Menge aller Punkte in der schraffierten Linse in Abbildung 3.1 korrespondiert direkt zu der Menge aller Punkte unter der Linie $\mathrm{AA}^{\prime}$ im positiven Quadranten von Abbildung 4.1. Diese umfaßt alle Umweltkostenvorteile der beiden Regierungen, die bei einer Ausgleichszahlung $z=0$ erreichbar sind. Bei einem Angebot $\hat{a}=\left(\hat{e}_{1}, \hat{e}_{2}, 0\right)$ würde eine Umweltkostensenkung $\Pi^{1}(\hat{a})=\Pi^{2}(\hat{a})=0$ erzielt. Somit entspricht das Gleichgewicht $\left(\hat{\mathrm{e}}_{1}, \hat{\mathrm{e}}_{2}\right)$ in Abbildung 3.1 dem Ursprung von Abbildung 4.1. Davon ausgehend, entspricht einer Bewegung auf der $\underline{\mathbf{k}}_{1}$-Isokostenlinie vom Gleichgewichtspunkt bis zum Schnittpunkt mit der Linie der Paretooptima in Abbildung 3.1 einer Bewegung auf der $\pi_{2}$-Achse vom Ursprung bis zum Punkt $A^{\prime}$ in Abbildung 4.1. Das gilt ebenso für die $\underline{k}_{2}$-Isokostenlinie und den Achsenabschnitt 0A. Weiterhin korrespondieren die Punkte auf der Linie der Paretooptima im Bereich der schraffierten Linse (den "Kernallokationen") zu der Linie AA' in Abbildung 4.1.

Ein entsprechender Vergleich ist zwischen Abbildung 3.2 und 4.2 für den Oberlieger-Unterlieger Fall möglich. Das Gleichgewicht $\left(\hat{\mathrm{e}}_{1}, \hat{\mathrm{e}}_{2}\right)$ in Abbildung 3.2 korrespondiert zu dem Ursprung in Abbildung 4.2. Eine Bewegung auf der Reaktionslinie $R^{2}\left(e_{1}\right)$ vom Gleichgewichtspunkt zur $e_{2}$-Achse, dem geometrischen Ort aller paretooptimalen Punkte, entspricht einer Bewegung auf der Linie 0A. Darin wird deutlich, daß, solange keine Ausgleichszahlungen zugelassen sind, eine Senkung des Oberlieger-Emissionsniveaus $e_{1}$ immer zu einer Erhöhung von dessen Umweltkosten führt und somit II $^{1}$ negativ wird. Erst Ausgleichszahlungen ermöglichen positive Umweltkostenvorteile für beide Umweltregionen. 


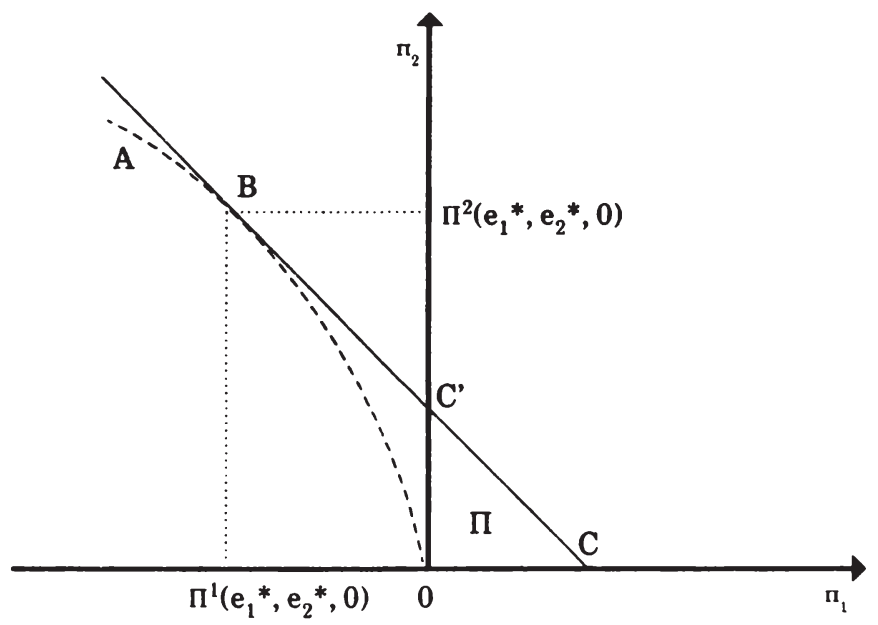

Abbildung 4.2

\subsubsection{Effiziente Umweltallokationen}

Die effizienten Emissionsbeschränkungen und Ausgleichszahlungen aus der Sicht der Gesamtökonomie bestimmen sich aus der Lösung des ParetoProblems

$$
\begin{aligned}
& \operatorname{Max} \Pi^{1}\left(e_{1}, e_{2}, z\right), \\
& e_{1}, e_{2}, z \\
& \Pi^{2}\left(e_{1}, e_{2}, z\right) \geq n^{\prime} .
\end{aligned}
$$

Die notwendigen Bedingungen zu der Lagrangefunktion L,

$$
L=\Pi^{1}\left(e_{1}, e_{2}, z\right)+\lambda\left[\Pi^{2}\left(e_{1}, e_{2}, z\right)-n^{\prime}\right]
$$


bestimmen die Lösung des Optimierungsproblems, wobei $\mathrm{A}$ eine nichtnegative Lagrangevariable ist. Aufgrund der Konkavität von $\Pi^{1}$ und $\Pi^{2}$ sind diese auch gleichzeitig hinreichend. Diese Bedingungen lauten:

$$
\begin{aligned}
& L_{e_{1}}=\Pi_{{ }^{1} e_{1}}+\lambda \Pi^{2}{ }_{e_{2}}=0, \\
& L_{e_{2}}=\Pi_{{ }^{1}}{ }_{e_{2}}+\lambda \Pi^{2}{ }_{e_{2}}=0, \\
& L_{z}=\Pi^{1}{ }_{z}+\lambda \Pi^{2}{ }_{z}=0 .
\end{aligned}
$$

Berücksichtigt man, daß $\Pi^{1}{ }_{2} \equiv 1$ und $\Pi^{2}{ }_{2} \equiv-1$ ist und folglich gemäß der letzten von den drei notwendigen Bedingungen $\lambda=1$ gilt, dann vereinfachen sich diese zu

$$
\begin{aligned}
& \Pi_{\mathrm{e}_{1}}^{\mathrm{i}}+\Pi_{\mathrm{e}_{1}}^{2}=0, \\
& \Pi_{\mathrm{e}_{2}}^{1}+\Pi_{\mathrm{e}_{2}}^{2}=0 .
\end{aligned}
$$

Diese notwendigen Bedingungen definieren ein Paar von Emissionsbeschränkungen $\left(e_{1}{ }^{*}, e_{2}{ }^{*}\right)$ mit der Eigenschaft:

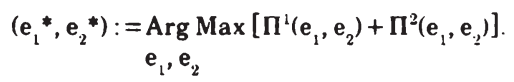

Somit ist bei der Möglichkeit von Ausgleichszahlungen eine paretooptimale Umweltallokation immer dadurch gekennzeichnet, daß der in (4.2) definierte Effizienzgewinn $\Pi$ maximiert wird. Berücksichtigt man die Definitionen von $\Pi^{1}$ und $\Pi^{2}$, werden damit gleichzeitig die aggregierten Umweltkosten $K^{1}+K^{2}$ minimiert. Einsetzen der effizienten Emissionsbeschränkungen $\left(e_{1}{ }^{*}, e_{2}{ }^{*}\right)$ in $(4.2)$ beschreibt den maximale Effizienzgewinn $\Pi^{*}$ :

$$
\begin{aligned}
\Pi^{*}\left(e_{1}^{*}, e_{2}^{*}\right) & \equiv \Pi^{1}\left(e_{1}^{*}, e_{2}^{*}, z\right)+\Pi^{*}\left(e_{1}^{*}, e_{2}^{*}, z\right) \\
& \equiv \underline{k}_{1}+\underline{k}_{2}-K^{1}\left(e_{1}^{*}, e_{2}^{*}\right)-K^{2}\left(e_{1}^{*}, e_{2}^{*}\right) .
\end{aligned}
$$


In den Abbildungen 4.1 und 4.2 trifft dieses bei einer Ausgleichszahlung $\mathrm{z}=0$ für den Punkt $\mathrm{B}$ auf der Linie $\mathrm{AA}^{\prime} \mathrm{zu}$. Alle weiteren effizienten Punkte liegen auf der Linie $\mathrm{CC}^{\prime}$. Sie sind ausgehend von der Kombination $\left(e_{1}{ }^{*}, e_{2}{ }^{*}, 0\right)$ durch eine entsprechende Umverteilung des maximalen Effizienzgewinns erreichbar. Ist beispielsweise $z=k_{2}-K^{2}\left(e_{1}{ }^{*}, e_{2}{ }^{*}\right)$, dann erhält Land $1 \mathrm{bzw}$. Umweltregion 1 den gesamten Effizienzgewinn. Dieser Situation entspricht Punkt $C$ in beiden Abbildungen.

Es läßt sich zeigen, daß der Effizienzgewinn $\Pi^{*}$ mit sinkenden Assimilationskräften des Umweltsystems zunimmt. Dazu differenziert man (4.7) nach $a_{1}$ und $a_{2}$, wobei man berücksichtigen muß, daß $\underline{k}_{1}=K^{1}\left(\hat{e}_{1}, \hat{e}_{2}\right)$ und $\underline{\mathrm{k}}_{2}=\mathrm{K}^{2}\left(\hat{\mathrm{e}}_{1}, \hat{\mathbf{e}}_{2}\right)$. Für die Ableitungen gilt

$$
\begin{aligned}
& \frac{\partial \Pi^{*}}{\partial a_{1}}=\left(\left(1-\beta_{1}\right) \hat{e}_{1}+\beta_{2} \hat{e}_{2}\right) D_{s}^{1}\left(S^{1}\left(\hat{e}_{1}, \hat{e}_{2}\right)\right)-\left(\left(1-\beta_{1}\right) e_{1}^{*}+\beta_{2} e_{1}{ }_{1}^{*}\right) D_{s}^{1}\left(S^{1}\left(e_{1}^{*}, e_{2}^{*}\right)\right)>0, \\
& \frac{\partial \Pi^{*}}{\partial a_{2}}=\left(\beta_{1} \dot{e}_{1}+\left(1-\beta_{2}\right) \hat{e}_{2}\right) D_{s}^{2}\left(S^{2}\left(\hat{e}_{1}, \hat{e}_{2}\right)\right)-\left(\beta_{1} e_{1}^{*}+\left(1-\beta_{2}\right) e_{1}^{*}\right) D_{s}^{2}\left(S^{2}\left(e_{1}^{*}, e_{2}^{*}\right)\right)>0 .
\end{aligned}
$$

Diese sind positiv, da erstens gemäß (3.11) und (3.12) die gesamtkostenminimalen Emissionsbeschränkungen $\left(e_{1}{ }^{*}, e_{2}{ }^{*}\right)$ strikt kleiner als die des Gleichgewichts bei unkoordinierter Umweltregulierung $\left(\hat{\mathrm{e}}_{1}, \hat{\mathrm{e}}_{2}\right)$ sind. Zweitens gilt aufgrund der strengen Konvexität der Schadensfunktion

$$
D_{s}^{\mathrm{i}}\left(\mathrm{S}^{\mathrm{i}}\left(\hat{\mathrm{e}}_{1}, \hat{\mathrm{e}}_{2}\right)>\mathrm{D}_{\mathrm{s}}^{\mathrm{i}}\left(\mathrm{S}^{\mathrm{i}}\left(\mathrm{e}_{1}^{*}, \mathrm{e}_{2}^{*}\right)\right) \quad(\mathrm{i}=1,2)\right.
$$

Bei sinkenden Assimilationskräften in den Umweltregionen, d.h. steigendem $a_{1}$ bzw. $a_{2}$, vergrößert sich der mögliche Effizienzgewinn. Damit erhöht sich gleichzeitig für die Regierungen der Anreiz miteinander zu verhandeln.

Eine Variation der Verteilungskoeffizienten $\beta_{1}$ und $\beta_{2}$ führt dagegen zu keinen eindeutigen Änderungen des Effizienzgewinns. Entsprechendes Ableiten von $\Pi^{*}$ nach $\beta_{1}$ und $\beta_{2}$ ergibt 


$$
\begin{aligned}
& \frac{\partial \Pi^{*}}{\partial \beta_{1}}=a_{2}\left(\hat{e}_{1} D_{s}^{2}\left(S^{2}\left(\hat{e}_{1}, \hat{e}_{2}\right)\right)-e_{1}^{*} D_{s}^{2}\left(S^{2}\left(e_{1}^{*}, e_{2}^{*}\right)\right)-a_{1}\left(\hat{e}_{1} D_{s}^{1}\left(S^{1}\left(\hat{e}_{1}, \hat{e}_{2}\right)\right)-e_{1}^{*} D_{s}^{1}\left(S^{1}\left(e_{1}^{*}, e_{2}^{*}\right)\right)\right.\right. \\
& \frac{\partial \Pi^{*}}{\partial \beta_{2}}=a_{1}\left(\hat{e}_{2} D_{s}^{1}\left(S^{1}\left(\hat{e}_{1}, \hat{e}_{2}\right)\right)-e_{2}^{*} D_{s}^{1}\left(S^{1}\left(e_{1}^{*}, e_{2}^{*}\right)\right)-a_{2}\left(\hat{e}_{2} D_{s}^{2}\left(S^{2}\left(\hat{e}_{1}, \hat{e}_{2}\right)\right)-e_{2}^{*} D_{s}^{2}\left(S^{2}\left(e_{1}^{*}, e_{2}^{*}\right)\right)\right.\right.
\end{aligned}
$$

Da mit den gleichen Argumenten wie eben die Klammerausdrücke positiv sind, läßt sich aus den Ableitungen nur schließen, daß bei einer stärkeren Verteilungswirkung des Umweltsystems ein Anstieg der Effizienzgewinne eher möglich ist, wenn die ausländischen Assimilationskräfte klein, dagegen die inländischen groß sind.

\subsubsection{Präferenzen der Regierungen}

Verhandlungen und ihre Ergebnisse werden von den Regierungen 1 und 2 jeweils anhand einer gegebenen Präferenzordnung bewertet. Sie erfassen damit u.a. Risiken, die dem Verhandlungs- und Einigungsprozeß innewohnen. Entscheidungsgrundlage einer Regierung i für die Wahl einer Verhandlungsstrategie mit dem Ziel, einen Anteil am Effizienzgewinn $n$ zu erhalten, ist der erreichbare Nutzen $w_{i}$. Dazu existiere für jede Regierung eine monoton steigende, konkave und differenzierbare vonNeumann-Morgenstern-Nutzenfunktion $W^{i}$,

$$
\mathbf{w}_{\mathrm{i}}=\mathbf{W}^{\prime}(\mathbf{n}) \text {, }
$$

als eine Funktion des Umweltkostenvorteils $\pi^{4}{ }^{4} W^{i}(0):=w_{i}$ ist dabei der Nutzen, den eine Regierung der Status-quo-Situation beimißt.

4) Dazu sei angemerkt, daß kooperative Verhandlungstheorien wie die Nash-Lösung nicht auf einer ordinalen Präferenzordnung aufbauen können, da sie interpersonelle Nutzenvergleiche erfordern. Shubik (1982, 92ff.) nennt diese auf Shapley (1969) zurūckgehende Erkenntnis das "Bargainers' Paradox". 
Die Funktion (4.8) definiert uns in Verbindung mit (4.3) im Rahmen des statischen Verhandlungsmodells die Menge $W$ aller in Verhandlungen potentiell einigungsfähigen Nutzenaufteilungen $w_{1}$ und $w_{2}$ der beiden Regierungen,

$$
W:=\left\{\left(W^{1}\left(\Pi^{1}(a)\right), W^{2}\left(\Pi^{2}(a)\right)\right) \mid a \in A\right\} .
$$

Aufgrund der Kompaktheit und Konvexität der Menge $A$ und der Konkavität der Funktionen $\Pi^{i}$ und $W^{i}(i=1,2)$ ist die Menge $W$ ebenfalls kompakt und konvex.

\subsection{Die verallgemeinerte Nash-Verhandlungslösung}

Die axiomatischen Teilungstheorien, denen das Nash-Verhandlungsmodell zugeordnet werden kann, gehen davon aus, daß sich jedes Verhandlungsproblem als ein Problem der Nutzenzuweisung auf der Basis bestimmter Regeln beschreiben läßt, ohne den eigentlichen Verhandlungsproze $ß$ explizit zu modellieren.

Mit Hilfe dieser als Postulate formulierten Regeln werden, neben Anforderungen an den imaginären Verhandlungsproze $\beta$, im wesentlichen zwei Ziele verfolgt. Zum einen lassen sich damit Forderungen an ein gewisses wünschenswertes Verhandlungsverhalten bzw. an das daraus resultierende Ergebnis stellen. Zu Forderungen dieses Typs gehören beispielsweise ein faires Verhalten in Verhandlungen oder ein gerechter Interessenausgleich zwischen den Verhandlungspartnern. Zum zweiten versucht man damit, wirkliche Verhandlungssituationen approximativ zu erklären. Axiome, die diesen Zweck erfüllen, müssen mit Annahmen über ein tatsächlich beobachtetes Verhalten vereinbar sein. Die Annahme eines individuell-rationalen Verhaltens oder der Versuch, die relative Verhandlungsstärke der Teilnehmer abzubilden, sind Beispiele dafür. Im 
ursprünglichen Nash-Modell steht der erste Punkt im Vordergrund, ${ }^{51}$ im Rahmen unseres Verhandlungsmodells dagegen der zweite. ${ }^{61}$

\subsubsection{Die Annahmen des verallgemeinerten Nash-Modells}

Die spieltheoretische Ausgangsbasis des statisch-axiomatischen NashVerhandlungsmodells ist die eines kooperativen Spiels in Normalform. ${ }^{7}$ ) Bezogen auf das Verhandlungsproblem der beiden Regierungen, wählen diese einmalig, simultan und unabhängig voneinander ein Nutzenniveau $w_{1}$ bzw. $w_{2}$. Diesen Nutzenniveaus liegen implizit bestimmte Verhandlungsstrategien für den gesamten Verhandlungsverlauf zugrunde. In unserem Fall wären dies Strategien mit Folgen von Angeboten aus den Angebotsmengen $A^{1}$ und $A^{2}$.

Das Problem der Einigung wird in diesem Modell, wie in allen kooperativen Modellen, stark vereinfacht. Ist die gewählte Kombination $w=\left(w_{1}, w_{2}\right)$ ein Element der Menge der einigungsfähigen Nutzenaufteilungen $W$, $w=\left(w_{1}, w_{2}\right) \in W$, dann gelten die Aufteilungen $w_{1}$ und $w_{2}$ als gegenseitig kompatibel. Damit wird unterstellt, daß eine Einigung zustandegekommen ist. $\mathrm{Zu}$ dieser Nutzenkombination existiert gemäß der Definition der Menge W (mindestens) eine Kombination von Emissionsbeschränkungen und einer Ausgleichszahlung $\left(e_{1}, e_{2}, z\right)$, die Inhalt eines internationalen Umweltabkommens sein können.

5) Axiome des ursprünglichen Nash-Verhandlungsmodells (Nash (1950)), die diesen Zweck verfolgen, sind das Axiom für Pareto-Optimalität und für Symmetrie.

6) Munro (1979) und Pethig (1982) orientieren sich in ihren statisch-axiomatischen Umweltverhandlungsmodellen an den ursprünglichen Nash-Axiomen.

7) Bei der Normalform eines Spiels umfaßt die Strategie eines Spielers den vollständigen Spielplan, d.h. alle Angaben, wie er in jeder möglichen Situation des gesamten Spielverlaufs reagieren wird. Das kooperative Spielkonzept läßt zu, daß die Teilnehmer außerhalb der durch die Spielregeln bestimmten formalen Struktur des Spiels miteinander reden, Vereinbarungen treffen, Drohungen übermitteln usw. 
Liegt die Kombination $w$ dagegen außerhalb der Menge $W, w=\left(w_{1}, w_{2}\right) \in W$, dann gelten die Nutzenaufteilungen $w_{1}$ und $w_{2}$ als inkompatibel und eine Einigung kommt nicht zustande. In diesem als Konfliktsituation bezeichneten Fall erreichen beide Regierungen nur den Nutzen $\underline{w}_{1}=W^{1}(0)$ bzw. $\underline{\mathrm{w}}_{2}=\mathrm{W}^{2}(0)$ des Status quo.

Durch die Menge $W$ einigungsfähiger Nutzenaufteilungen und die Nutzenaufteilung $\underline{w}=\left(\underline{w}_{1}, \underline{w}_{2}\right)$ für die Konfliktsituation ist das Verhandlungsproblem vollständig beschrieben. Eine Nash-Lösung dieses Problems ist ein Funktion $\mathbf{F}$ : $(\boldsymbol{W}, \underline{\mathbf{w}}) \rightarrow \mathbf{R}^{2}$, die diesen Bestimmungsgrößen einen Wert $w^{*}=\left(w_{1}^{*}, w_{2}^{*}\right)$, den Nutzen der beiden Regierungen im Verhandlungsgleichgewicht, zuweist. Somit gilt

$$
\mathbf{w}^{*}=\mathbf{F}(\mathbf{W}, \underline{\mathbf{w}}) \text {, }
$$

wobei $w_{1}^{*}=F^{1}(W, \underline{w})$ und $w_{2}{ }^{*}=F^{2}(W, \underline{w})$.

Eine Lösung $F(W, \underline{w})$ des durch $(W, \underline{w})$ beschriebenen Verhandlungsspiels sollte in Hinblick auf unser Ziel, reale Verhandlungssituationen zu erklären, drei als Axiomen formulierten Eigenschaften genügen:

1. (Individuelle Rationalität) Für jede Verhandlungslösung $F$ gelte $F^{1}(W, \underline{w}) \geq \underline{w}_{1}$ und $F^{2}(W, \underline{w}) \geq \underline{w}_{2}$.

2. (Unabhängigkeit von gleichwertigen Nutzendarstellungen) Für alle $a_{i}, b_{i} \in \mathbf{R}, a_{i}>0(i=1,2)$ sei das linear transformierte Verhandlungs. $\operatorname{spiel}\left(W^{\prime}, \underline{w}^{\prime}\right)$ definiert durch

$$
W^{\prime}=\left\{\left(y_{1}, y_{2}\right) \mid y_{t}=a_{i} x_{i}+b_{t}(i=1,2),\left(x_{1}, x_{2}\right) \in W\right\}
$$

und $u_{i}=a_{i} \underline{w}_{i}+b_{i}$, dann gelte $F^{\prime}\left(W^{\prime}, \underline{w}^{\prime}\right)=a_{i} F^{i}(W, \underline{w})+b_{i}$ für $i=1,2$. 
3. (Unabhängigkeit von irrelevanten Alternativen) $E_{s}$ sei $W \subset W$ und $F(W, \underline{w}) \in W^{\prime}$, dann gelte $F(W, \underline{w})=F(W, \underline{w})$.

Axiom 1 fordert von den Regierungen ein individuell-rationales Verhalten im klassischen Sinn, wonach nur solche Nutzenaufteilungen als kompatibel gelten, die den Nutzen eines jeden Landes gegenüber der jeweiligen Status-quo-Situation nicht verringern. Diese Eigenschaft ist schon bei der Definition der Menge $W$ berücksichtigt. ${ }^{81}$

Axiom 2 bezieht sich auf die Eigenschaften und damit auf die Informationen, die in der von-Neumann-Morgenstern-Nutzenfunktion enthalten sind. Aus der Nutzentheorie wissen wir, daß eine positive lineare Transformation die mit einer solchen Nutzenfunktion beschriebene Präferenzordnung nicht beeinflußt. ${ }^{\text {' }}$ Das gleiche wird für die Lösung des Verhandlungsspiels gefordert. Eine Änderung der Nutzenskala einer oder beider Regierungen läßt somit die Lösung unverändert.

Während die Axiome 1 und 2 Bedingungen an den äußeren Rahmen des Verhandlungsspiels und an das Verhalten der beiden Regierungen darstellen, bezieht sich Axiom 3 dagegen mehr auf den Typ von Verhandlungen, den das Modell abbildet. Es fordert, daß die Lösung bei einer Vergrößerung der Menge einigungsfähiger Nutzenaufteilungen entweder unverändert bleibt oder gleich einer der neu hinzugekommenen Nutzenaufteilungen ist. ${ }^{101}$

Axiom 3 läßt sich in zweifacher Hinsicht interpretieren. Erstens, nach Roth (1979, 7), modelliert es implizit einen Verhandlungsprozeß, der sich

8) Dieses Axiom ersetzt die restriktive Pareto-Optimalitätshedingung im ursprünglichen Nash-Verhandlungsmodell, mit der a priori unterstellt wird, daß sich beide Regierungen ausschließlich auf effiziente Ergebnisse einigen. Beispielsweise läßßt dieses Axiom nicht zu, daß sich beide Verhandlungsparteien möglicherweise nicht einigen. Auf diese Weise soll das dadurch geforderte kollektivistische Verhalten im gemeinsamen Interesse durch ein stärker an eigennützigen Zielen orientiertes Verhalten ersetzt werden.

9) Vergleiche dazu beispielsweise Shubik $(1982,92)$.

10) Vergleiche dazu Roth $(1979,7)$. 
zunächst einmal näherungsweise an einer kleineren Menge W' von alternativen Nutzenaufteilungen orientiert und dazu eine Lösung sucht. Danach bestimmt man die Lösung für die Gesamtmenge $W$ der Alternativen nur noch in einem Vergleich zwischen der Lösung aus der kleineren Menge und den neu hinzugekommenen Elementen aus der Differenzmenge WW'. Eine zweite Interpretationsmöglichkeit ergibt sich, wenn man anstatt der Vergrößerung von einer Verkleinerung der Menge einigungsfähiger Nutzenaufteilungen ausgeht. Würde man von dieser Menge einige Elemente ausschließen, die sowieso nicht von den Regierungen gewählt worden wären, dann wird eine Lösung von diesen unbedeutenden Alternativen nicht beeinflußt.

\subsubsection{Das verallgemeinerte Nash-Verhandlungsgleichgewicht}

Roth $(1979,14 \mathrm{ff}$ ) hat gezeigt, daß genau zwei Nash-Lösungen F existieren, die diese drei Axiome erfüllen. ${ }^{11}$ 'Die eine ist der Nichteinigungsfall

$$
F(W, \underline{w})=\left(\underline{w}_{1}, \underline{w}_{2}\right) \text {. }
$$

Die zweite Lösung wird in Verbindung mit (4.1) und (4.8) durch die Lösung des Optimierungsproblems

$$
F(W, \underline{w})=\underset{a \in A}{\operatorname{ArgMax}}\left[\mathbf{W}^{1}\left(\Pi^{1}(a)\right)-\underline{w}_{1}\right]^{\gamma}\left[W^{2}\left(\Pi^{2}(a)\right)-\underline{w}_{2}\right]^{1-\gamma}
$$

spezifiziert, wobei $y$ ein beliebiger Parameter, $0 \leq Y \leq 1$, ist. Die "verallgemeinerte Nash-Verhandlungslösung", wie sie von Binmore (1980) genannt wird, besitzt damit die Eigenschaft, ein gewichtetes geometrisches Mittel der Nutzenzuwächse gegenüber dem Status quo zu maximieren.

11) Den Existenz- und Eindeutigkeitsbeweis stellen wir an dieser Stelle nicht dar. Er kann bei Roth $(1979,14 f$ f.) als Beweis zu den Theoremen 2 und 3, aufbauend auf dem Beweis für das ursprüngliche Nash-Theorem nachgelesen werden. Vergleiche dazu auch Kalai (1977) und Binmore (1980). 
Ihre Eigenschaften werden durch die notwendigen Bedingungen des Optimierungsproblems in (4.10) spezifiziert. Diese lauten

$$
\begin{aligned}
& W_{n}^{1} \Pi^{1}{ }_{e_{1}} \gamma\left[W^{1}-\underline{w}_{1}\right]^{Y-1}\left[W^{2}-\underline{w}_{2}\right]^{1-\gamma} \\
& +W_{n}^{2} \Pi_{e_{1}}^{2}(1-\gamma)\left[W^{2}-\underline{w}_{2}\right]^{-\gamma}\left[W^{1}-\underline{w}_{1}\right]^{\gamma}=0, \\
& W_{n}^{1} \Pi^{1} e_{2} Y\left[W^{1}-\underline{w}_{1}\right]^{\gamma-1}\left[W^{2}-\underline{w}_{2}\right]^{1-\gamma} \\
& +W^{2}{ }_{n} \Pi_{e_{2}}^{2}(1-\gamma)\left[W^{2}-\underline{w}_{2}\right]^{-v}\left[W^{1}-\underline{w}_{1}\right] v=0, \\
& \mathrm{~W}^{1}{ }_{\mathrm{n}} \boldsymbol{\Pi}^{1}{ }_{2} \mathrm{Y}\left[\mathrm{W}^{1}-\underline{\mathbf{W}}_{1}\right]^{\mathrm{Y}-1}\left[\mathrm{~W}^{2}-\underline{\mathbf{W}}_{2}\right]^{1-\mathrm{Y}} \\
& +W^{2} \Pi^{2}{ }_{z}(1-\gamma)\left[W^{2}-\underline{w}_{2}\right]^{-\gamma}\left[W^{1}-\underline{w}_{1}\right]^{\gamma}=0,
\end{aligned}
$$

bzw. nach Umformen

$$
W_{n}^{i} \Pi_{e_{i}}^{i}\left[W^{2}-\underline{w}_{2}\right]+W_{n}^{2} \Pi_{e_{1}}^{2}(1-\gamma)\left[W^{1}-\underline{w}_{1}\right]=0,
$$

$$
W_{n}^{i} \Pi_{e_{2}, y}^{1}\left[W^{2}-\underline{w}_{2}\right]+W_{n}^{2} \Pi_{e_{2},}^{2}(1-\gamma)\left[W^{1}-\underline{w}_{1}\right]=0,
$$

$$
\mathrm{W}_{\mathrm{n}}^{1} \mathrm{II}_{2}^{1} \mathrm{Y}\left[\mathrm{W}^{2}-\underline{w}_{1}\right]+\mathrm{W}^{2}{ }_{\mathrm{n}} \Pi^{2}{ }_{2}(1-\mathrm{y})\left[\mathrm{W}^{1}-\underline{\mathrm{w}}_{1}\right]=0 \text {. }
$$

Berücksichtigt man, daß gemäß der Definition in (4.1) $\Pi^{1}{ }_{2} \equiv 1$ und $\Pi^{2}{ }_{2} \equiv-1$, dann vereinfacht sich (4.13) zu

$$
W_{n}^{1} Y\left[W^{2}-\underline{W}_{2}\right]-W^{2}{ }_{n}(1-Y)\left[W^{1}-\underline{W}_{1}\right]=0 \text {. }
$$

Umformen ergibt

$$
\frac{y W_{n}^{1}}{(1-y) W_{n}^{2}}=\frac{W^{1}\left(\Pi^{1}(a)\right)-w_{1}}{W^{2}\left(\Pi^{2}(a)\right)-w_{2}}
$$

Einsetzen von (4.14) in (4.11) und (4.12) führt zudem auf 
(4.16)

$$
\begin{aligned}
& \Pi_{\mathrm{e}_{1}}^{1}+\Pi^{2} \mathrm{e}_{1}=0 \\
& \Pi^{\mathrm{l}} \mathrm{e}_{2}+\Pi^{2}{ }_{\mathrm{e}_{2}}=0 .
\end{aligned}
$$

Diese Bedingungen sind vollkommen identisch zu (4.5).

Durch die Bedingungen (4.15) und (4.16) wird die verallgemeinerte NashVerhandlungslösung vollständig charakterisiert. Gemäß (4.16) ist bei dem von den Axiomen 1-3 geforderten Verhalten und bei Zulassen von Ausgleichszahlungen eine gesamtkostenminimale Umweltallokation $\left(\mathrm{e}_{1}{ }^{*}, \mathrm{e}_{2}{ }^{*}\right)$ erreicht; mithin der internationale externe Effekt vollkommen internalisiert. Die notwendige Bedingung in (4.15) stellt dazu eine Regel dar,

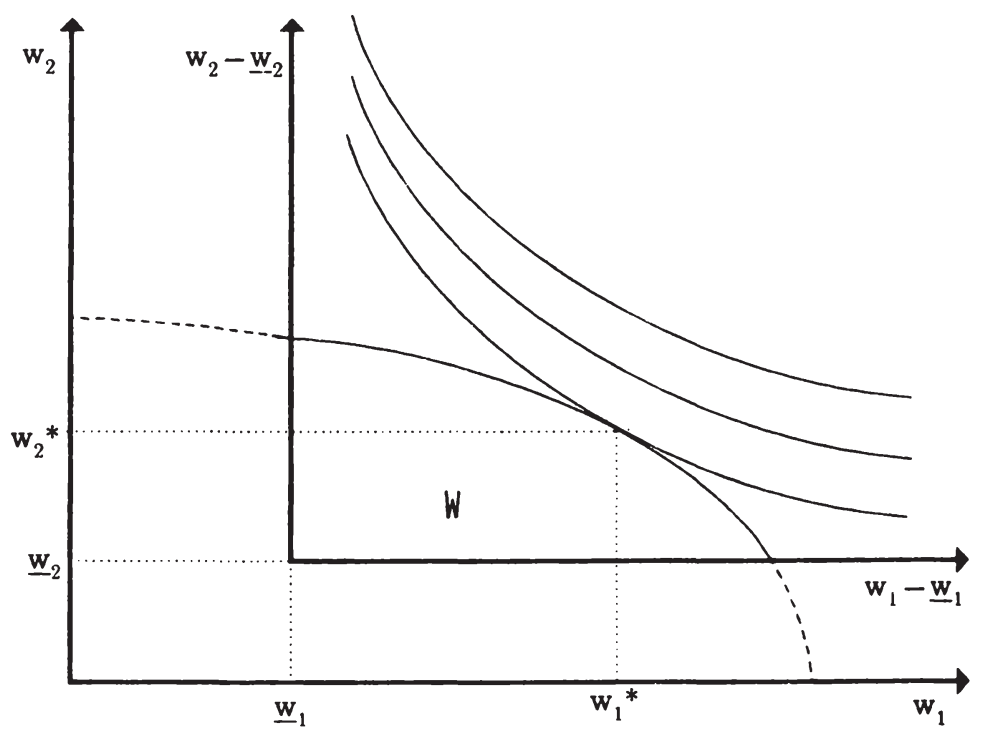

Abbildung 4.3 
wie der bei dieser Allokation maximale Effizienzgewinn $\Pi *\left(e_{1}{ }^{*}, e_{2}{ }^{*}\right)$ zwischen den beiden Ländern bzw. Umweltregionen verteilt werden soll. Das geschieht danach in der Weise, daß das Verhältnis der gegenüber dem Status quo erreichten Nutzenzuwächse dem mit y bzw 1-y gewichteten Verhältnis der Grenznutzen entspricht.

Abbildung 4.3 zeigt graphisch die Nutzenverteilung $\left(w_{1}{ }^{*}, w_{2}{ }^{*}\right)$ bzw. die Verteilung der Nutzenzuwächse $\left(w_{1}^{*}-\underline{w}_{1}, w_{2}^{*}-\underline{w}_{2}\right)$ im Verhandlungsgleichgewicht. Diese wird als Tangentialpunkt einer Kurve an den Rand der Menge W dargestellt. Jede Kurve aus der dargestellten Schar repräsentiert den geometrischen Ort aller Kombinationen von Nutzenzuwächsen für einen vorgegebenen Wert der Zielfunktion in (4.10) und gegebenen Parameter y. Verändert man $y$, führt das zu einer Verschiebung der Kurven-Schar, folglich verschiebt sich der Gleichgewichtspunkt ebenfalls auf dem Rand der Menge W.

Aus der notwendigen Bedingung in (4.15) läßt sich ersehen, daß durch eine entsprechende Wahl von $\gamma$ jeder Punkt auf dem paretooptimalen Rand der Menge $W$ erreicht werden kann. Der Wert des Parameters y hat somit einen entscheidenden Einfluß auf die Verteilung der Effizienzgewinne. Die unserem Modell zugrunde liegenden Axiome 1-3 können aber diesen Wert nicht erklären, folglich muß y durch ein zusätzliches Axiom oder exogen bestimmt werden.

Nash (1950) hat zu diesem Zweck in seinem ursprünglichen Modell das Symmetrie-Axiom eingeführt. Es lautet in unserem Modellzusammenhang:

4. (Symmetrie) Wenn $\underline{w}_{1}=\underline{w}_{2}$ und wenn für jedes $\left(w_{1}, w_{2}\right) \in W$ gilt $\left(w_{2}, w_{1}\right) \in W$, dann ist $F^{1}(W, \underline{w})=F^{2}(W, \underline{w})$.

Dieses Axiom fordert, da $\beta$ das Verhandlungsergebnis für beide Regierungen gleich sein muß, solange sie sich nicht in ihren Ausgangsbedingungen unterscheiden. Damit baut es prinzipiell auf dem gleichen Argument auf, das Axiom 2 zugrunde liegt: Eine Verhandlungslösung 
darf nur von der im Modell enthaltenen Information abhängen, d.h. den Präferenzen der Regierungen über einigungsfähige Allokationen in Form der Menge Wund dem Nutzenniveau w im Konfliktfall.

Legt man Axiom 4 zusammen mit den Axiomen 1-3 dem Verhandlungsmodeil zugrunde, ergibt sich als Lösung die ursprüngliche NashLösung $\mathrm{F}^{\mathrm{N}}$ :

$$
F^{N}(W, \underline{w})=\underset{a \in f}{\operatorname{ArgMax}}\left[W^{1}\left(\Pi^{1}(a)\right)-\underline{w}_{1}\right]\left[W^{2}\left(\Pi^{2}(a)\right)-\underline{w}_{j}\right] .
$$

Diese ist von den Ergebnissen her gleichwertig zu dem Maximum der Zielfunktion in (4.10) für den Parameterwert ${ }_{Y}=1 / 2 .{ }^{121}$

Will man unterschiedliche Ausgangsbedingungen der Teilnehmer in Verhandlungen modellieren, die über die bisherige Modellbeschreibung hinausgehen, dann wäre es unsinnig, dies mit Axiomen zu tun. Axiome haben die Funktion allgemeiner und damit für jedermann gleichermaßen gültiger Regeln, die für solche $\mathrm{Z}$ wecke somit ungeeignet sind. Als Alternative bietet sich nur die Möglichkeit, diese zusätzliche Information aggregiert über eine exogene Bestimmung des Parameters y in das Modell einzuführen.

Zu Ausgangsbedingungen einer Regierung, die das Modell zunächst nicht abbilden kann, zählen beispielweise das Drohpotential in Form der Oberlieger- oder der Unterliegerposition im Umweltsystem, der innenpolitische Druck ein bestimmtes Ergebnis im Inland vorweisen zu müssen, der Informationsstand, taktisches Geschick, usw. Ausgangsbedingungen dieser Art subsummiert man oft unter dem Begriff "Verhandlungsmacht". Der Parameter $y$ würde in diesem Zusammenhang als Maß dienen, um die Auswirkungen von Verhandlungsmacht $z u$ beschreiben. Je größer y umso höher ist in unserem Modell für Land 1 bzw. Umweltregion 1 der Anteil

12) Vergleiche dazu Theorem 2 bei Roth $(1979,14)$. Die Gleichwertigkeit ergibt sich daraus, daß mit $Y=1 / 2$ die ursprüngliche Nash-Zielfunktion in (4.17) durch eine positiv-monotone Transformation erreicht wird, ohne die Struktur der Funktion zu verändern. 
am gesamten Effizienzgewinn. Damit verbindet man, daß mit steigendem y die Verhandlungsmacht von Regierung 1 im Verhältnis zu Regierung 2 steigt. ${ }^{131}$

Der große Nachteil dieser Vorgehensweise ist, daß man mit der exogenen Vorgabe des Parameters $y$ wohl unterschiedliche Ausgangsbedingungen empirisch beschreiben, aber die Auswirkungen solcher Phänomene auf das Verhandlungsergebnis aus dem Modell heraus nicht erklären kann.

\subsection{Die Grenzen des statisch-axiomatischen Nash-Modells}

Die Diskussion im vorausgegangenen Abschnitt hat schon angedeutet, daß der statisch-axiomatische Ansatz zur Beschreibung von Verhandlungssituationen Schwächen aufweist. Dazu lassen sich einige Kritikpunkte anführen. Einer davon, auf den schon im vorangegangenen Abschnitt hingewiesen wurde, bezieht sich auf die beschränkte Infomation zur Beschreibung der Verhandlungssituation. Der statisch-axiomatische Ansatz nutzt nur die Informationen, die in den Präferenzen der Regierungen über einigungsfähige Umweltallokationen und in dem Nutzenniveau des Konfliktfalls enthalten sind. Damit klammert man die Einflußgrößen von Verhandlungsergebnissen aus, die in Unvollkommenheiten des Verhandlungsmarktes und den darauf ablaufenden Verhandlungsprozessen stecken.

Ein zweiter Kritikpunkt bezieht sich darauf, daß sich ein individuellrationales Verhalten nur eingeschränkt, d.h. nur im klassischen Sinn, abbilden läßt. Damit kann ein strategisches Verhalten der Verhandlungsparteien, beispielsweise eine optimale Reaktion auf mögliche Unvollkommenheiten wie asymmetrische Information überhaupt nicht analysiert werden.

13) Vergleiche dazu auch Binmore, Rubinstein, Wolinsky (1985). 
Ein dritter Kritikpunkt an den statisch-axiomatischen Ansätzen bezieht sich auf ihre Formulierung als kooperatives Spiel. Die Anwendung dieses Konzepts wird ab dem Punkt problematisch, wo es um die Verteilung des möglichen Effizienzgewinns geht. Die Regierungen befinden sich dort in genau gegensätzlichen Positionen zueinander und damit in einer typischen nichtkooperativen Verhandlungssituation. Unabhängig davon, ob eine Lösung endogen unter Zugrundelegung des Symmetrie-Axioms oder exogen durch die Vorgabe des Paramters y bestimmt wird, unterstellt der Aufbau des Spiels implizit ein kooperativ-faires Verhalten der Regierungen bei der Gewinnverteilung. Die im Symmetrie-Axiom des ursprünglichen Nash-Modells enthaltene oder durch eine exogene Bestimmung von $\mathrm{y}$ vorgegebene Aufteilungsregel ist im Sinne eines Schiedsspruchs zu verstehen, der von den Regierungen in jedem Fall als gerecht empfunden und akzeptiert wird. ${ }^{14}$

Nash (1953) versuchte sich diesem letzten Kritikpunkt zu entziehen und hat gezeigt, daß sich die axiomatische Lösung als ein Grenzwert einer Folge von Gleichgewichten nichtkooperativer Spiele darstellen läßt, wenn die Spieler im Konfliktfall einseitig erreichbare Allokationen als sogenannte "Drohpunkte" beziehen. Damit wird das gesamte Verhandlungsspiel in zwei Teilspiele zerlegt, ein nichtkooperatives Spiel um die optimalen Drohpunkte und das anschließende kooperative Spiel, welches die Gewinnverteilung festlegt. Bei dieser "Konstruktion" findet der eigentliche Verhandlungsprozeß im nichtkooperativen Spiel statt. In dieser Phase besteht das Ziel jedes Spielers darin, bei gegebenem Verhalten des anderen und gegebener Gewinnverteilung, den Drohpunkt so zu wählen, daß der Nutzenzuwachs bei der Gewinnverteilung maximal wird.

Das Drohkonzept erweitert wohl den Umfang individuell-rationalen Verhaltens, es beseitigt aber nicht die wesentlichen Kritikpunkte am Nash-Modell. Die Schwäche dieses Konzepts liegt wie im ursprünglichen Modell darin, daß es sich nur auf den Konfliktfall bezieht und nicht auf das Verhalten im eigentlichen Verhandlungsprozeß. Ein zusätzlicher

14) In der Literatur wird die Nash-Lösung daher oft als "Schiedsrichterlösung" bezeichnet. 
Kritikpunkt ergibt sich dadurch, daß bei Drohstrategien kein Vergleich mit der besten Alternative außerhalb der Verhandlungen stattfindet. Sind die Opportunitätskosten von Drohstrategien relativ hoch, werden Drohstrategien unglaubhaft und damit unsinnig.

\subsection{Zusammenfassung}

Die Ergebnisse des ersten Abschnitts in diesem Kapitel bestätigen die Ergebnisse in der Literatur, wonach sich durch eine Kooperation bei grenzüberschreitenden externen Effekten eine gegenüber dem unkoordinierten Fall paretosuperiore Umweltallokation erzielen läßt. Um jedoch diese Effekte vollständig zu internalisieren, d.h. eine Allokation zu erreichen, die die aggregierten Umweltkosten der beiden Länderökonomien minimiert, sind Kompensationszahlungen notwendig. Das betrifft insbesondere den Oberlieger-Unterlieger-Fall. Hierbei kommt bei einem individuell-rationalen Verhalten der Regierungen ohne solche Zahlungen überhaupt keine Kooperation zustande.

Eine internationale Zusammenarbeit setzt Verhandlungen zwischen den beiden Länderregierungen mit dem Ziel voraus, die Regulierungspolitiken für die beiden Umweltregionen aufeinander abzustimmen. Dieses Verhandlungsproblem wird, um an die Ausgangsbedingungen des "CoaseTheorems" anknüpfen zu können, mit Hilfe eines statisch-axiomatischen Ansatzes, einer verallgemeinerten Version des kooperativen NashVerhandlungsmodells, beschrieben. Die zugrundegelegten Axiome modellieren einen vollkommenen Verhandlungsmarkt, auf dem den Regierungen keinerlei Transaktionskosten entstehen. Zudem stellt die Annahme der Unabhängigkeit von irrelevanten Alternativen nur minimale Anforderungen an den Ablauf des möglichen Verhandlungsprozesses.

Das Modell abstrahiert von dem restriktiven Axiom des Nash-Verhandlungsmodells, das a priori ein paretooptimales Verhandlungsergebnis voraussetzt. Dem entspricht die Annahme des "Coase-T'heorems", daß sich 
die Verhandlungsparteien ausschließlich im gemeinsamen Interesse und nicht eigennützig verhalten. Anstatt dessen wird ein individuell-rationales Verhalten im klassischen Sinn angenommen.

Unter den gegebenen Präferenzen der beiden Regierungen und den Axiomen wird entweder ein Verhandlungsergebnis erreicht, welches auf dem paretooptimalen Rand der Menge möglicher Aufteilungen des Effizienzgewinns liegt, oder es kommt zur Nichteinigung. Das Modell läßt aber im Fall des effizienten Ergebnisses unbestimmt, welche Gewinnaufteilung sich ergibt. Solange Verhandeln mit keinerlei Kosten verbunden ist, kann jedes effiziente Ergebnis erreicht werden.

Die bisherige Analyse des Verhandlungsproblems im Rahmen des statisch-axiomatischen Ansatzes erscheint unbefriedigend. Modelltheoretisch betrachtet bietet dieses Modell kein klares Kriterium, anhand dessen man entscheiden kann, welches die "richtige" Lösung ist. Das von Nash in seinem ursprünglichen Modell unterstellte Symmetrie-Axiom und ebenso die exogene Vorgabe des "Verhandlungsmacht"-Parameters $Y$ sind mehr im Sinn eines salomonischen Urteils aufzufassen, als eine saubere theoretische Erklärung dafür, mittels welcher Kriterien die Verhandlungsparteien rational unter den möglichen Lösungen eine davon auswählen.

Realistischerweise ist zudem Verhandeln nicht kostenlos, d.h. die Beteiligten wenden im Verlaufe eines Verhandlungsprozesses Ressourcen auf, um ein bestimmtes Ergebnis zu erzielen. Beispielsweise abstrahiert man bei einer statischen Formulierung des Verhandlungsproblems von Transaktionskosten, die dadurch entstehen, daß während der Verhandlungszeit weiterhin eine gesamtökonomisch ineffiziente Umweltnutzung stattfindet oder dadurch, daß Regierungen für die Informationsbeschaffung zu den Verhandlungen Kosten entstehen. Damit stellt sich die Frage, inwieweit nicht die daraus resultierende Unvollkommenheit auf Verhandlungsmärkten Antworten liefern, welches Verhandlungsergebnis gewählt wird. Eine Hypothese ist, da $\beta$ die Regierung mit den höheren Transaktionskosten einen geringeren Anteil am Effizienzgewinn erhält. 
Aus modelltheoretischer wie auch aus ökonomischer Sicht besteht somit die Forderung; die Verhandlungssituation im Rahmen eines dynamischen Modells zu formulieren, das zum einen ein individuell-rationales Verhalten in Verhandlungen abbilden kann und zum anderen den Einbezug von spezifischen Transaktionskosten erlaubt. Möglichkeiten dazu bieten uns die dynamisch-strategischen Ansätze in der neueren Theorie der Verhandlungsspiele. Sie unterscheiden sich vom statisch-axiomatischen Ansatz in der Weise, daß der Verhandlungsproze $B$ darin explizit modelliert wird. ${ }^{14}$ Dabei vereinen sie kooperative Lösungen mit nichtkooperativen Lösungen.

14) Der Begriff "dynamisch-strategischer Ansatz" kennzeichnet die Eigenschaft des Verhandlungsspiels, alle im Verlauf des Spiels getroffenen Entscheidungen explizit darzustellen. Es ist nicht gleichzusetzen mit "strategischer Form" eines Verhandlungsspiels. Strategische Ansätze können sowohl in extensiver als auch in strategischer (Normal-) Form eines Spiels beschrieben sein. 



\section{Die dynamisch-strategische Lösung bei koordinierter Umweltnutzung}

In diesem Kapitel erweitern wir das bisher statisch analysierte Verhandlungsproblem zwischen den beiden Länderregierungen, indem in einem nichtkooperativen Modell ein Verhandlungsprozeß über die Zeit explizit dargestellt wird. Gegenseitige Verhandlungsangebote oder Reaktionen auf Angebote werden als Züge in einem nichtkooperativen MehrperiodenSpiel betrachtet. Die kooperativen Elemente, wie der Ablauf des Verhandlungsprozesses und eine mögliche Einigung auf ein für beide Regierungen verpflichtendes Abkommen, sind genau in den Regeln des nichtkooperativen Spiels festgelegt. Darüber hinausgehende bindende Vereinbarungen läßt die nich tkooperative Formulierung nicht zu.

Dieser sogenannte dynamisch-strategische Ansatz ermöglicht ein individuell-rationales Verhalten der Regierungen analog zu den Annahmen in Abschnitt 3.2.1. darzustellen. Damit können nun klar die Einflüsse von Unvollkommenheiten des Verhandlungsmarktes und von institutionellen Randbedingungen der Verhandlungen, wie die rechtliche Ausgangssituation oder asymmetrische Information, analysiert werden. Desweiteren erlaubt die dynamische Formulierung, den Einfluß von Veränderungen der Kosten-Nutzen-Strukturen zu untersuchen, die mit der Zeit in den Umweltregionen eintreten können, wie mit der Zeit zunehmende Schäden aufgrund abnehmender Assimilationskräfte des Umweltsystems. Diese Einflußgrößen bestimmen weitgehend die individuellen Transaktionskosten einer Regierung und damit deren relative Verhandlungsposition.

Der Vorteil dieser Vorgehensweise liegt darin, daß sich auf diese Weise ein eindeutiges Verhandlungsgleichgewicht bestimmen läßt. Er muß aber dadurch erkauft werden, daß wir uns auf eine genau spezifizierte, regelmäßige Struktur des Verhandlungsprozesses festlegen müssen. ${ }^{1}$

1) Ein solches Vorgehen wird von Crawford (1985) aber nicht als wesentliche Einschränkung einer Modellanalyse gesehen: "Something must be specified exogenously,..., it seems a sensible research strategy to begin with the simplest rules of bargaining that are rich enough to address the issues of interest." 
Nach der Definition des dynamischen Verhandlungsmodells in Abschnitt 5.1. analysieren wir dieses im Abschnitt 5.2. zunächst als ein nichtkooperatives Mehrperioden-Verhandlungsspiel mit unendlichem Zeithorizont und mit volllkommener Information der beiden Regierungen. Als Konzept zur Bestimmung von Lösungen dieses Spiels dient das teilspielperfekte Nash-Gleichgewicht. Es erscheint als das natürliche Lösungskonzept für sequentiell ablaufende Verhandlungsprozesse. Individuelle Rationalität einer Regierung impliziert danach, daß ihr Verhalten in jeder möglichen Verhandlungsrunde optimal in Hinblick auf den weiteren Verhandlungsverlauf sein muß. Die Gleichgewichtsbetrachtung dieses dynamischstrategischen Modells wird durch einen Vergleich zu dem statischaxiomatischen Ansatz aus Kapitel 4., sowie in Abschnitt 5.3. durch eine komparativ-statische Analyse erweitert. Dabei wird gezielt der Einfluß zeitwirksamer Parameter des Modells untersucht.

Der unendliche Zeithorizont des Modells, sowie die Annahme vollkommener Information erleichtern die Einführung des speziellen Gleichgewichtskonzepts, stellen aber gleichzeitig einschränkende Annahmen dar. Ergänzend wird daher in Abschnitt 5.4. untersucht, wie sich die Verhandlungsergebnisse verändern, wenn die Umgebungsbedingungen in den beiden Umweltregionen im Zeitverlauf diskreten Variationen unterworfen sind. Wir trennen dazu zwischen Veränderungen, die genau vorhersehbar sind und solchen, die vorhersehbar, aber deren Zeitpunkt des Eintritts unsicher ist. Abschnitt 5.5. faßt die Ergebnisse des Kapitels abschließend zusammen.

\subsection{Das dynamische Verhandlungsmodell}

Die zentrale Annahme des dynamischen Verhandlungsmodells ist die Vorgabe einer genau spezifizierten Struktur des Verhandlungsprozesses, in der die Abfolge von Angeboten sowie Reaktionen auf Angebote für beide Regierungen vollständig beschrieben wird. 
Der mögliche Verhandlungsproze $\beta$ besteht aus einzelnen Verhandlungsrunden, die regelmäßig und in gleichen Zeitabständen stattfinden. Damit wird ein gleichmäßiges Zeitraster in das Modell eingeführt, dessen Periodenlänge durch den zeitlichen Abstand zwischen zwei Verhandlungsrunden definiert wird. Dadurch können wir größtenteils auf die Definitionen des statischen Modells zurückgreifen, indem die in (3.4) definierten Umweltkosten $\mathrm{K}^{1}$ und $\mathrm{K}^{2}$ als diejenigen Kosten betrachtet werden, die in einer Umweltregion pro Periode entstehen. Gleiches gilt entsprechend für die in (4.1) definierten Umweltkostenvorteile $\Pi^{1}$ und $\Pi^{2}$.

Vereinfachend gelte zunächst, daß bei gegebenen Emissionsbeschränkungen die Umweltkosten pro Periode keinen exogenen Änderungen im Zeitverlauf unterworfen und damit Umweltkosten wie auch Umweltkostenvorteile stationär sind. Darnit lassen sich die Verhandlungen von heute über eine Umweltallokation morgen gleichermaßen auf alle zukünftigen Perioden übertragen. Somit können wir davon ausgehen, daß ein zustande gekommenes Abkommen in seiner zeitlichen Gültigkeit unbefristet ist.

\subsubsection{Verhandlungsstruktur}

Zur Modellierung der genauen Struktur des Verhandlungsprozesses in den einzelnen Verhandlungsrunden und zwischen den Runden folgen wir einem erstmals von Stahl (1972) und später wieder von Rubinstein (1982), Binmore (1982), Shaked und Sutton (1982) sowie Cramton (1985) verwendeten Ansatz mit einem alternierenden Angebots- und Reaktionsprozeß.

Dieser Proze $ß$ hat folgenden Ablauf. Initiiert beispielsweise Regierung 1 die Verhandlungen, dann gibt diese am Anfang der ersten Periode $t=0$ in einer ersten Verhandlungsrunde ein Angebot $a_{0}=\left(e_{10}, e_{20}, z_{0}\right)$ ab, wobei dieses Angebot ein Element aus ihrer in (4.2) definierten individuellen Angebotsmenge $A^{1}$ ist. Regierung 2 muß sich noch in der gleichen Verhandlungsrunde entscheiden, ob sie das Angebot $a_{0}$ annimmt oder es 
ablehnt. Nimmt sie es an, gilt ein Abkommen auf der Basis dieses Angebots als zustandegekommen und tritt sofort in Kraft. Der Verhandlungsproze $\beta$ wird dadurch beendet. Lehnt sie dagegen das Angebot $a_{0} a b$, gibt sie selbst am Anfang der nächsten Periode $t=1$ in einer zweiten Verhandlungsrunde ein Gegenangebot $a_{1}=\left(e_{11}, e_{21}, z_{1}\right)$ aus ihrer individuellen Angebotsmenge $A^{2}$ ab. Auf dieses Angebot muß wiederum Regierung 1 durch Annahme oder Ablehnung reagieren, usw. In jeder Periode findet somit höchstens eine Verhandlungsrunde statt und in jeder Verhandlungsrunde wird höchstens ein Angebot abgegeben und es erfolgt die dazugehörige Reaktion in Form von Annahme oder Ablehnung.

Solange der Verhandlungsprozeß andauert, wechselt somit von Verhandlungsrunde zu Verhandlungsrunde die Rolle des Anbieters und desjenigen, der auf das Angebot reagieren muß. Dabei wird unterstellt, daß die Regierungen an ihre vorhergehenden Angebote nicht gebunden sind. Auch die mögliche Höchstdauer des Prozesses ist nicht beschränkt. Jeder kann solange weiterverhandeln, wie er sich noch zu verbessern glaubt, so da $ß$ fur den Periodenindex $t$ gilt $t \in \mathbb{N}$. Beginnt Regierung 1 in Periode $t=0$ die Verhandlungen, dann ist sie, solange der Verhandlungsproze $\beta$ andauert, in jeder Periode mit geradem Periodenindex $t$ am Zuge, ein Angebot abzugeben und entsprechend Regierung 2 in jeder Periode mit ungeradem Index $t$.

Die beiden Regierungen besitzen vollkommene Information und damit eine sichere Voraussicht darauf, zu welchen zukünftigen Konsequenzen ihre jeweiligen Angebots- und Reaktionsentscheidungen führen. Ihnen sei ein individuell-rationales Verhalten im Sinne der Annahmen in Abschnitt 3.2.1. unterstellt, wonach eine Regierung das zukünftige Verhalten der anderen Regierung antizipiert und in ihren eigenen Entscheidungen mit berücksichtigt.

Gleichsam besitzen die Regierungen bei ihren Entscheidungen ein vollständiges Erinnerungsvermögen. Somit wird die Entscheidung über ein Angebot $a_{t}$ unter Berücksichtigung der bisher abgegebenen und abgelehnten Angebote $a_{0}, \ldots, a_{t-1}$ getroffen. Die Entscheidung der Regierung i 
$(i=1,2)$ über ein Angebot $a_{t}$ ist somit eine Funktion $\left\{a_{0}, \ldots, a_{t-1}\right\} \rightarrow A i$. Ebenso werden bei der Entscheidung über Annahme und Ablehnung des Angebots $a_{t}$ das Angebot $a_{t}$ selbst, aber auch die bisher abgelehnten Angebote mit in das Entscheidungskalkül einbezogen. Damit ist die Entscheidung über Annahme oder Ablehnung eines Angebots $a_{t}$ eine binäre Funktion $\left\{a_{0}, \ldots, a_{t}\right\} \rightarrow\{j a$, nein $\}$.

Jeder im Sinne dieser Regeln mögliche Verhandlungsprozeß wird für eine Regierung vollständig durch eine Folge dieser Funktionen beschrieben. Diese Folgen stellen Vorschriften für die Regierungen dar, wie sie sich in Abhängigkeit von dem vorangegangenen Verhandlungsverlauf in jeder stattfindenden Verhandlungsrunde zu verhalten haben. Spieltheoretisch gesprochen sind solche Folgen reine Verhandlungsstrategien einer Regierung. Zur formalen Definition aller möglichen Verhandlungsprozesse und im Hinblick auf unsere Gleichgewichtsbetrachtung im Rahmen eines Verhandlungsspiels wollen wir dafür diesen Begriff beibehalten.

Es sei $F$ die Menge aller Verhandlungsstrategien von Regierung 1 und entsprechend $G$ die Menge aller Verhandlungsstrategien von Regierung 2. $F$ und $G$ enthalten alle Folgen von Funktionen $F=\left\{F^{t}\right\}_{t}=0,1,2, \ldots$ und $G=\left\{G^{t}\right\}_{t}=0,1,2, \ldots$ für alle Perioden $t=0,1, \ldots, \infty$. Die Funktionen $F^{t}$ und $G^{t}$ besitzen bei Beginn der Verhandlungen durch Regierung 1 für gerade $t$ die Eigenschaft

$$
\begin{aligned}
& F^{t}:\left\{a_{0}, \ldots, a_{t-1}\right\} \rightarrow A^{1}, \\
& G^{t}:\left\{a_{0}, \ldots, a_{t}\right\} \rightarrow\{j a, \text { nein }\}
\end{aligned}
$$

und für ungerade t die Eigenschaft

$$
\begin{aligned}
& F^{t}:\left\{a_{0}, \ldots, a_{t}\right\} \rightarrow\{\text { ja, nein }\}, \\
& G^{t}:\left\{a_{0}, \ldots, a_{t-1}\right\} \rightarrow A^{2} .
\end{aligned}
$$

Beginnt dagegen Regierung 2 die Verhandlungen, wechseln die Indizes und es gilt für gerade $t$ 


$$
\begin{aligned}
& \mathbf{F}^{\mathrm{t}}:\left\{\mathbf{a}_{0}, \ldots, \mathbf{a}_{\mathrm{t}}\right\} \rightarrow\{\text { ja, nein }\}, \\
& \mathbf{G}^{\mathrm{t}}:\left\{\mathbf{a}_{0}, \ldots, \mathbf{a}_{\mathrm{t}-1}\right\} \rightarrow \mathrm{A}^{2},
\end{aligned}
$$

und für ungerade $t$

$$
\begin{aligned}
& \mathrm{F}^{\mathrm{t}}:\left\{\mathrm{a}_{0}, \ldots, \mathrm{a}_{\mathrm{t}-1}\right\} \rightarrow \mathrm{A}^{\mathrm{t}}, \\
& \mathrm{G}^{\mathrm{t}}:\left\{\mathrm{a}_{0}, \ldots, \mathrm{a}_{\mathrm{t}}\right\} \rightarrow\{\text { ja, nein }\} .
\end{aligned}
$$

Dazu ein Beispiel: $\mathrm{G}^{1}\left(\mathrm{a}_{0}, \bar{a}_{1}\right)$ ist die ja-oder-nein-Antwort der beginnenden Regierung 2, nachdem ihr erstes Angebot $a_{0}$ in der Periode $t=0$ von Regierung 1 abgelehnt wurde und Regierung 1 nun selbst in Periode $t=1$ ein Gegenangebot $a_{1}$ unterbreitet hat.

Es werde von Regierung 1 eine Strategie $F \in F$ und von Regierung 2 eine Strategie $G \in G$ gewählt. Unter diesen Bedingungen sind im Verlaufe des gesamten Prozesses zwei verschiedene Folgen von Ergebnissen in den einzelnen Verhandlungsrunden möglich: Entweder es kommt nach einer Folge abgelehnter Angebote $a_{0}, \ldots, a_{t-1}$ in Periode $t$ über ein Angebot $a_{t} \mathbf{z u}$ einer Einigung, womit der Verhandlungsprozeß endet, oder die Folge der abgelehnten Angebote ist unendlich, und es wird keine Einigung erreicht. In diesem letzten Fall bleiben die beiden Länder in ihrer Status-quoSituation.

\subsubsection{Zeitpräferenzen der Regierungen}

Die dynamische Struktur des Verhandlungsprozesses erfordert den Faktor Zeit in die Bewertung der während des Verhandlungsprozesses abgegebenen Angebote einzubeziehen. Bewertungs- und Entscheidungsgrundlage für die Regierungen 1 und 2 über ein am Anfang der Periode $t$ abgegebenes Angebot $a_{t}$ ist der jeweilige Nutzen aus dem Strom von Umweltkostenvorteilen $\Pi^{1}\left(a_{t}\right)$ und $\Pi^{2}\left(a_{t}\right)$, die diese ausgehend von der aktuellen Verhandlungsrunde in allen zukünftigen Perioden realisieren können. 
Dazu definieren wir zunächst für beide Regierungen Zeitpräferenzordnungen $\geq_{1}$ und $\geq_{2}$, die ermöglichen, dem im Zeitpunkt $l\left(l \in \mathbf{R}_{+}\right)$ erreichten Umweltkostenvorteil $n$, geschrieben $(n, 1)$, einen auf den Zeitpunkt l $=0$ normierten Nutzen zuzuordnen. Diese Zeitpräferenzordnungen sollen folgenden sechs Eigenschaften genügen:

1. $\geq_{i}$ ist eine schwache Präferenzordnung auf $\Pi \times \mathbf{R}_{+}$.

2. (Nichtsättigung) Wenn $\pi^{\prime}>n^{\prime \prime}$, dann gilt $\left(n^{\prime}, l^{\prime}\right)>\left(n^{\prime \prime}, l^{\prime}\right)$.

3. (Stetigkeit) $\left\{\left(n^{\prime}, l^{\prime}\right) /\left(n^{\prime}, l^{\prime}\right) \geq\left(n^{\prime \prime}, l^{\prime \prime}\right)\right\}$ und $\left\{\left(n^{\prime}, l^{\prime}\right) /\left(n^{\prime \prime}, l^{\prime \prime}\right) \geq\left(n^{\prime}, l^{\prime}\right)\right\}$ sind abgeschlossene Mengen.

4. (Monotonie der Zeitpräferenz) Wenn $l^{\prime}<l^{\prime \prime}$, dann gilt für $\pi^{\prime}>0$ $\left(n^{\prime}, l^{\prime}\right) \geq_{\mathrm{i}}\left(\pi^{\prime}, l^{\prime \prime}\right)$ und für $\pi^{\prime}=0\left(\pi^{\prime}, l^{\prime}\right) \sim_{i}\left(\pi^{\prime}, l^{\prime \prime}\right)$.

5. (Konkave Kompensation) Für $l^{\prime}<l^{\prime \prime}$ sei eine Funktion $\Psi$ definiert durch $\left(n^{\prime}, l^{\prime}\right) \sim_{i}\left(n^{\prime}+\Psi\left(n^{\prime}\right), l^{\prime \prime}\right)$, dann ist $\Psi$ steigend und konkav .

6. (Stationarität) Für ein $\varepsilon \in\{0,1,2 \ldots\}$ gelte $\left(n^{\prime} \cdot l^{\prime}\right) \sim\left(n^{\prime \prime}, l^{\prime}+\varepsilon \tau\right)$, dann impliziert das $\left(\pi^{\prime}, l^{\prime \prime}\right) \sim_{i}\left(n^{\prime \prime}, l^{\prime \prime}+\varepsilon \imath\right)$.

Die Eigenschaften 1-3 entsprechen den allgemein üblichen Eigenschaften einer Präferenzordnung, wie sie aus der Konsumtheorie bekannt sind. Bei den Eigenschaften 4-6 tritt der Zeitaspekt stärker in den Vordergrund. Wir wollen daher kurz darauf eingehen.

Eigenschaft 4 fordert einen monotonen Trade-off zwischen einem Umweltkostenvorteil heute und morgen. Der Vorteil von heute wird dem gleichen Vorteil morgen vorgezogen. Eigenschaft 5 erfaßt den Einfluß von Risikoaversion über die Zeit. Darin läßt sich die Funktion $\Psi$ als ein Äquivalent für den Nutzenverlust über die Zeit interpretieren. Dieses Äquivalent muß mit steigendem Umweltkostenvorteil ebenfalls zunehmen. Bei einer stetigen Variation der Zeit impliziert diese Eigenschaft die konvexe Krümmung der Indifferenzkurven in einer Umweltkostenvorteil-Zeit-Ebene. Eigenschaft 6 wird später entscheidend für die Gleichgewichtsbeschreibung des Verhandlungsproblems sein. Ihre wesentliche 
Aussage ist, daß eine Indifferenz zwischen $z w e i$ zu verschiedenen Zeitpunkten anfallenden Umweltkostenvorteilen nur von der Zeitdifferenz zwischen den beiden Realisierungszeitpunkten abhängt und nicht von den absoluten Zeitpunkten selbst. Eine gleichmäßige Verschiebung beider Realisierungszeitpunkte würde die Indifferenz somit erhalten.

Fishburn und Rubinstein (1982) haben gezeigt, daß sich eine Zeitpräferenzordnung $\geq_{i}$ mit diesen Eigenschaften durch eine von-NeumannMorgenstern-Nutzenfunktion $U^{* i}(n, l)$ der Form

$$
\mathrm{L}^{* *}(n, \mathrm{l})=\mathrm{V}(n) \mathrm{e}^{-\mathrm{r}_{\mathrm{i}} \mathrm{l}} \quad(\mathrm{i}=1,2)
$$

repräsentieren läßt. Darin ist $V^{i}$ eine monoton steigende stetige und konkave Funktion des Umweltkostenvorteils a und $r_{i}$ eine marginale Diskontrate mit der Eigenschaft $r_{i} \geq 0$.

Aufgrund der festen Periodeneinteilung im Verhandlungsmodell läßt sich das Zeitmaß 1 durch die von Randbedingungen der Verhandlungen festgelegte Zeitdifferenz $\tau\left(\tau \in \mathbb{R}_{+}\right)$zwischen zwei Verhandlungsrunden und dem Periodenindex $t(t \in\{0,1,2, \ldots\})$ beschreiben. Demzufolge ist

$$
\mathrm{I}=\mathrm{t} \mathrm{t} .
$$

Mit (5.1) gilt nun für den Nutzen $u_{1}$ bzw. $u_{2}$ der Regierungen, wenn sie in Periode t einen Umweltkostenvorteil $n$ realisieren

$$
\mathrm{u}_{\mathrm{i}}=\mathrm{V}^{\prime}(\mathrm{n}) \delta_{1}^{\mathrm{i}}
$$

Dabei ist $\delta_{i}$ ein von der Periodenlänge $\tau$ und der marginalen Diskontrate $\mathbf{r}_{i}$ abhängiger Diskontfaktor, definiert durch

$$
\delta_{i}:=e^{-r_{i}} \boldsymbol{t},
$$

der im Rahmen der Gleichgewichtsanalyse zunächst die Schreibweise vereinfacht. 
Wenn sich die beiden Regierungen im Verlaufe des Verhandlungsprozesses über einem Angebot einigen, realisieren sie jeweils ab dem Einigungszeitpunkt in der gegenwärtigen und allen zukünftigen Perioden einen entsprechend ausgehandelten Umweltkostenvorteil. Wir müssen daher für unsere Zwecke die Nutzen der ab dem Einigungszeitpunkt realisierten Umweltkostenvorteile über alle zukünftigen Perioden aggregieren.

Nach der Summenformel für eine unendliche geometrische Reihe würde jede Regierung bei sofortiger Einigung auf einen Vertrag in der ersten Runde der Verhandlungen einen zusätzlichen Nutzen in der Höhe des Gegenwartswerts aller zukünftigen nutzenindizierten Umweltkostenvorteile $n$,

$$
\mathrm{V}^{\mathrm{i}}(\mathrm{n}) \frac{1}{1-\delta_{\mathrm{i}}},
$$

realisieren. Nach der Summenformel für eine endliche geometrische Reihe entstehen dagegen jeder Regierung bei einer Nichteinigung bis einschließlich Periode t-1 Nutzenverluste in der Höhe

$$
\mathrm{V}^{\mathrm{i}}(\mathrm{n}) \frac{1-\delta_{i}^{\mathrm{t}}}{1-\delta_{\mathrm{i}}}
$$

Die Differenz aus diesen beiden Größen,

$$
V^{i}(\pi) \frac{1}{1-\delta_{i}}-V^{i}(\pi) \frac{1-\delta_{i}^{t}}{1-\delta_{i}}=V^{i}(\pi) \frac{\delta_{i}^{t}}{1-\delta_{i}},
$$

beschreibt den Gegenwartswert des Nutzenstroms aller zukünftigen Umweltkostenvorteile $n$, die ab Periode t realisiert werden.

Zusammen mit der Definition des Umweltkostenvorteils in (4.1) läßt sich somit der aggregierte Nutzen $U^{i}$,

$$
U^{i}\left(a_{t}, t\right):=V^{i}\left(\Pi^{i}\left(a_{t}\right)\right) \frac{\delta_{i}^{t}}{1-\delta_{i}} \quad(i=1,2),
$$


definieren, der sich mit einem in Periode $t$ abgegebenen Angebot $a_{t,}$, $a_{t}=\left(e_{1 t}, e_{2 t}, z_{t}\right)$, erzielen läßt. Die Nutzenfunktionen $U^{i}$ dienen im folgenden als Zielfunktionen zur Bewertung und Entscheidung über die im Verlaufe des Verhandlungsprozesses von den beiden Regierungen abgegebenen Angebote.

Die durch die Nutzenfunktion $U^{i}$ dargestellte Präfenzordnung erfaßt nicht nur die Einschätzung des Nichteinigungsrisikos wie im statischen Fall, sondern auch die Ungeduld und den Nutzenverlust einer Regierung, der bei einer verzögerten Einigung und dadurch verhinderter Effizienzgewinne entsteht. Der darin enthaltene Diskontfaktor $\delta_{i}$ besitzt inhaltlich eine Doppelfunktion. Er stellt zum einen ein Maß für eine geringere Bewertung zukünftiger Umweltkostenvorteile dar. Hier ist er aber auch ein Maß für den Druck, dem eine Regierung ausgesetzt ist, damit sie in einer bestimmten Zeit ein bestimmtes politisch gewünschtes Verhandlungsergebnis erzielt. Je größer die Zeitpräferenz ist, d.h. je kleiner $\delta_{i}$, umso "ungeduldiger" (Koopmans (1960)) ist eine Regierung.

\subsection{Das teilspielperfekte Nash-Gleichgewicht}

Die Mengen $F$ und $G$ der möglichen Verhandlungsstrategien, sowie die beiden Nutzenfunktionen $U^{1}$ und $U^{2}$ des dynamischen Verhandlungsmodells bilden die Ausgangsbedingungen eines nichtkooperativen Mehrperioden-Verhandlungsspiels in extensiver Form. Im Gegensatz zum statisch-axiomatischen Ansatz ist in diesem dynamisch-strategischen Ansatz nun genau geregelt, wie die möglichen Verhandlungsprozesse ablaufen können, sowie unter welchen Bedingungen und wann es zu einer Einigung kommen kann. Damit werden die implizit in einem kooperativen Spiel unterstellten, aber unsichtbaren Elemente bei dieser nichtkooperativen Formulierung sich tbar gemacht. 
Das zentrale Konzept zur Bestimmung von möglichen Lösungen dieses nichtkooperativen Verhandlungsspiels ist das nichtkooperative NashGleichgewichtskonzept. Der Grund dafür liegt darin, da $B$ nichtkooperative Spiele keine bindenden Vereinbarungen erlauben, die über die explizit in den Spielregeln zugelassenen hinausgehen. Somit müssen Lösungen des Spiels in der Weise selbsterhaltend und stabil sein, daß nach der Einigung auf ein Abkommen für keine Regierung Anreize zum einseitigen Vertragsbruch bestehen. Eben diese Eigenschaften erfüllt ein Nash-Gleichgewicht: Keine Regierung kann davon profitieren, wenn sie sich durch einseitigeWahl einer anderen als der Gleichgewichtsstrategie von dem Gleichgewichtspunkt wegbewegt.

Die direkte Anwendung des klassischen nichtkooperativen Nash-Gleichgewichtsbegriffs, wie wir ihn auch zur Beschreibung des Gleichgewichts bei unkoordinierter Umweltnutzung verwendet haben, wirft aber in unserem jetzigen Modell Probleme auf. Es erweist sich, daß dieser in seiner ursprünglichen Form für unser Mehrperioden-Verhandlungsspiel zu grob und damit ungeeignet ist. Die Anzahl der Lösungen, die danach als Nash-Gleichgewicht einzustufen wären, ist zu groß.

Betrachten wir beispielhaft eine Situation, in der Regierung 1 die Verhandlungen beginnt und ein erstes für sie vorteilhaftes und paretooptimales Angebot $a^{\prime}=\left(e_{1}{ }^{*}, e_{2}{ }^{*}, z^{\prime}\right)$ abgibt. Sie kündigt gleichzeitig an, in allen zukünftigen Verhandlungsrunden Gegenangebote abzulehnen, die die Umweltregion 1 gegenüber dem Angebot a' schlechter stellen, und selber immer das gleiche Angebot a' abzugeben. Glaubt Regierung 2 an die darin enthaltene Drohung, ist es für sie rational, dem Angebot a' von Regierung 1 sofort in der ersten Runde zuzustimmen, denn bei dieser Einschätzung kann sie sich in den weiteren Verhandlungsrunden nicht mehr verbessern. In diesem Sinne bilden die von Regierung 1 angekündigte Strategie und die daran angepaßte Strategie von Regierung 2 ein nichtkooperatives Nash-Gleichgewicht. Würde Regierung 2 jede Ankündigung dieser Art als glaubwürdig ansehen, dann bilden alle paretooptimalen Angebote ein Nash-Gleichgewicht. 
Die Problematik des klassischen nichtkooperativen Gleichgewichtsbegriffs liegt hier darin, inwieweit solche Ankündigungen glaubhaft sind und tatsächlich eine substantielle Drohung beinhalten. Das Beispiel vernachlässigt die Auswirkungen der sequentiellen zeitintensiven Struktur von Verhandlungen, in diesem Modell zusätzlich verstärkt durch den laufenden Wechsel der Anbieterrolle. Danach beinhalten nur solche Angebote von Regierung 1 eine glaubhafte Drohung für Regierung 2, bei denen sie sich nicht durch eine Ablehnung und ein entsprechendes Gegenangebot in der nachfolgenden Verhandlungsrunde und allen weiteren Runden verbessern kann. Das gleiche gilt analog für Regierung 1 bei Angeboten von Regierung 2.

Diese Überlegungen im Zusammenhang mit adäquaten Gleichgewichtsbegriffen für Mehrperiodenspiele dieses Typs führen auf das von Selten (1965), (1975) vorgeschlagene Konzept des teilspielperfekten Nash-Gleichgewichts. Bezogen auf unseren Verhandlungsprozeß läßt sich ein derartiges Gleichgewicht wie folgt definieren:

\section{Definition:}

Ein Gleichgewichtspunkt in reinen Strategien $\left(F^{*}, G^{*}\right)$ des in Periode $t=0$ beginnenden Mehrperioden-Verhandlungsspiels in extensiver Form gilt als teilspielperfekt, wenn in jedem seiner in Periode $t=1,2, \ldots$ beginnenden Teilspiele, die aus $\left(F^{*}, G^{*}\right)$ abgeleiteten Strategien Gleichgewichtspunkt dieses Teilspiels sind.

Die Gleichgewichtsstrategien $\left(\mathrm{F}^{*}, \mathrm{G}^{*}\right)$ in einem teilspielperfekten NashGleichgewicht besitzen damit eine Eigenschaft, die von Kreps und Wilson (1982) in einem allgemeineren Zusammenhang als "sequentiell rationales" Verhalten bezeichnet wird: Das Angebots- bzw. Reaktionsverhalten einer Regierung maximiert in jeder möglichen Verhandlungsrunde, unter Berücksichtigung des zukünftigen individuell-rationalen Verhaltens der anderen Regierung, ihren Nutzen in Hinblick auf den weiteren möglichen Verlauf der Verhandlungen. ${ }^{2}$

2) Damit läßt sich das teilspielperfekte Nash-Gleichgewicht als das spieltheoretische Gegenstück des Optimalitätsprinzips der dynamischen Programmierung auffassen. 
Rubinstein (1982) und ebenso Binmore (1982) haben die Existenz teilspielperfekter Nash-Gleichgewichte für Verhandlungsspiele des von uns unterstellten Typs mit alternierenden Angeboten gezeigt. ${ }^{31}$ Es ist daher ausreichend, die Gleichgewichte durch die notwendigen Bedingungen der Optimierungsprobleme beider Regierungen bei ihrer Angebotswahl zu charakterisieren. Diese Optimierungsprobleme erhalten aber durch den vorgegebenen Verhandlungsablauf mit alternierender Angebotsabgabe und Reaktion eine spezielle Struktur.

Dazu betrachten wir beispielhaft eine Situation, in der Regierung 1 die Verhandlungen in der ersten Runde mit einem ersten Angebot $a_{0}$ begonnen hatte und nach einer Folge bisher abgelehnter Angebote $a_{0}, \ldots, a_{t-1}$ im Zeitpunkt $t, t \in(0,2,4, \ldots)$, eine neues, im Sinne ihrer Zielsetzung optimales Angebot $a_{t}$ abgeben soll.

Die Verhandlungsregeln gewähren Regierung 1 für diese Runde eine Stackelberg-Unabhängigkeitsposition, denn sie wird bei der Angebotswahl nur durch das mögliche Reaktionsverhalten von Regierung 2 beschränkt. Regierung 2 befindet sich für diese Runde in der schwächeren Abhängigkeitsposition und muß sich dem Angebotsverhalten von Regierung 1 anpassen. Das Reaktionsverhalten von Regierung 2 ist nur in der Weise an das eigene Angebotsverhalten in der Periode $t+1$ gekoppelt, da $B$ sie ihre Möglichkeiten in Periode $t$ mit denen in Periode $t+1$ vergleicht. Sie wird nur solche Angebote $a_{t}$ von Regierung 1 annehmen, wenn sie in Periode $t+1$ kein für sie besseres Angebot $a_{t+1}$ abgeben kann, dem wiederum Regierung 1 im Hinblick auf den weiteren Verhandlungsverlauf zustimmen würde. In der Periode $t$ muß Regierung 1 , um eine Zustimmung von Regierung 2 zu erhalten, dieser somit mindestens den Nutzen

3) Die Existenzaussage wird in den Modellen von Kreps und Wilson (1982) durch Zulassen von Unsicherheit und der Erweiterung auf Verhaltensstrategien verallgemeinert. Fudenberg und Levine (1983) zeigen die Existenz in reinen und gemischten Strategien für Spiele mit endlicher Anzahl von Zügen und unendlichem Zeithorizont, wobei die vorher übliche Stationarität der Zeitpräferenz nicht erforderlich ist und simultane Züge zugelassen werden. Harris (1985) bestätigt in einer alternativen Modellbeschreibung die Ergebnisse von Fudenberg und Levine. 
zugestehen, den diese in der folgenden Verhandlungsrunde mit ihrem eigenen Angebot erzielt hätte.

Das optimale Angebot $a_{t}$ von Regierung 1 in Periode $t$ löst somit das Maximierungsproblem:

$$
\begin{aligned}
& \operatorname{Max}_{a_{t} \in A^{1}} U^{1}\left(a_{t}, t\right) \\
& U^{2}\left(a_{t}, t\right) \geq U^{2}\left(a_{t+1}, t+1\right) \quad\left(a_{t+1} \in A^{2}, t=0,2,4, \ldots\right) .
\end{aligned}
$$

Dabei erwartet Regierung 1 in Periode $t+1$ von Regierung 2 ein Angebot $a_{t+1}$, welches das Maximierungsproblem löst:

$$
\begin{aligned}
& \operatorname{Max}_{a_{t+1} \in A^{2}} U^{2}\left(a_{*+1}, t+1\right) \\
& \left.\left.C^{1}\left(a_{t+1}\right), t+1\right) \geq L^{-1}\left(a_{*+2}\right), t+2\right) \quad\left(a_{*+2} \in A^{1}, t=0,2,4, \ldots\right) .
\end{aligned}
$$

Die Lösung des Optimierungsproblems (5.4) läßt sich durch die notwendigen Bedingungen zur Lagrangefunktion L,

$$
\begin{aligned}
& L=V^{1}\left(\Pi^{i}\left(a_{:}\right)\right) \delta_{1}^{\prime}\left(1-\delta_{1}\right)^{-1} \\
& +\Lambda\left[\left(V^{2}\left(\Pi^{2}\left(a_{\imath}\right)\right) \delta_{2}^{\prime}\left(1-\delta_{2}\right)^{-1}-V^{2}\left(\Pi^{2}\left(a_{i+1}\right)\right) \delta_{2}^{:+1}\left(1-\delta_{i}\right)^{-1}\right],\right.
\end{aligned}
$$

charakterisieren, wobei $\lambda$ eine nichtnegative Lagrangevariable ist. Die notwendigen Bedingungen lauten:

$$
\begin{aligned}
& L_{e_{1}}=V_{n}^{1} \Pi_{e_{1}}^{1} \delta_{1}^{t}\left(1-\delta_{1}\right)^{-1}+\lambda V_{n}^{2} \Pi_{e_{1}}^{2} \delta_{2}{ }^{\prime}\left(1-\delta_{2}\right)^{-1}=0, \\
& L_{e_{2}}=V_{n}^{1} \Pi_{e_{2}}^{1} \delta_{!}^{t}\left(1-\delta_{1}\right)^{-1}+\lambda V_{n}^{2} \Pi_{e_{2}}^{2} \delta_{2}^{t}\left(1-\delta_{2}\right)^{-1}=0 \text {, } \\
& L_{z}=V_{n}^{1} \Pi^{1}{ }_{z} \delta_{1}^{*}\left(1-\delta_{1}\right)^{-1}+\lambda V_{n}^{2} \Pi^{2} \delta_{2}{ }^{\prime}\left(1-\delta_{2}\right)^{-1}=0 .
\end{aligned}
$$

Zudem gilt als Bedingung für den komplementären Schlupf

$$
\Lambda\left[\left(\mathrm{V}^{2}\left(\Pi^{2}\left(\mathrm{a}_{\mathrm{t}}\right)\right) \delta_{2}^{\mathrm{i}}\left(1-\delta_{2}\right)^{-1}-\mathrm{V}^{2}\left(\Pi^{2}\left(\mathrm{a}_{t+1}\right)\right) \delta_{2}^{:+1}\left(1-\delta_{2}\right)^{-1}\right]=0 .\right.
$$


Aufgrund der Konkavität von $\mathrm{V}^{1}, \Pi^{1}$ und $\mathrm{V}^{2} \circ \Pi^{2}$ in $\mathrm{e}_{1}, \mathrm{e}_{2}$ und $\mathrm{z}$ sind die notwendigen Bedingungen auch gleichzeitig hinreichend. Berücksichtigt man gemäß der Definition in (4.1), daß $\Pi^{1}{ }_{z} \equiv 1$ und $\Pi^{2}{ }_{z} \equiv-1$, dann vereinfacht sich (5.8) zu

$$
V_{n}^{1} \delta_{1}^{\prime}\left(1-\delta_{1}\right)^{-1}=\lambda V_{n}^{2} \delta_{2}^{*}\left(1-\delta_{2}\right)^{-1}
$$

Diese Gleichung in (5.6) und (5.7) eingesetzt führt zu

$$
\begin{aligned}
& \Pi_{e_{1}}^{1}+\Pi_{e_{1}}^{2}=0, \\
& \Pi_{e_{2}}^{1}+\Pi_{e_{2}}^{2}=0 .
\end{aligned}
$$

Abstrahiert man einmal von der Periodisierung der Effizienzgewinne in diesem Modell, dann ist diese Bedingung identisch mit (4.5). Somit enthält ein optimales Angebot $a_{t}$ von Regierung 1 immer einen Vorschlag für eine gesamtkostenminimale Umweltallokation $\left(e_{1 t}, e_{2 t}\right)=\left(e_{1}^{*}, e_{2}^{*}\right)$. Daraus können wir schon an dieser Stelle die Schlußfolgerung ziehen, daß trotz des unterstellten nichtkooperativen, nur auf den eigenen Vorteil bedachten Verhaltens solch ein Gleichgewichtsangebot impliziert, daß die internationalen externen Effekte vollkommen internalisiert werden.

Aufgrund der strengen Monotonie der Zielfunktion ist die Nebenbedingung im Optimum bindend und dadurch $\lambda>0$, so daß mit (5.9) unter Berücksichtigung von $\left(e_{1 t}, e_{2 t}\right)=\left(e_{1}{ }^{*}, e_{2}{ }^{*}\right)$ gilt:

$$
V^{2}\left(\Pi^{2}\left(e_{1}^{*}, e_{2}^{*}, z_{t}\right)\right)=V^{2}\left(\Pi^{2}\left(a_{t+1}\right)\right) \delta_{2} \quad(t=0,2,4, \ldots) .
$$

Für gegebenes $a_{t+1}$ ist dadurch die Höhe der optimalen Ausgleichszahlung $\mathrm{z}_{\mathrm{t}}$ eindeutig bestimmt.

Die Lösung des Optimierungsproblems (5.5) ist formal bis auf die veränderten Parameter und Indizes vollkommen analog zur Lösung des Problems in (5.4), so daß sich eine ausführliche Beschreibung des Lösungswegs erübrigt. Aus den notwendigen Bedingungen ergibt sich wiederum 
analog zu (5.10), daß $\left(e_{1 t+1}, e_{2 t+1}\right)=\left(e_{1}{ }^{*}, e_{2}{ }^{*}\right)$ gilt. Ebenso wird die optimale Ausgleichzahlung $\mathrm{z}_{\mathrm{t}+1}$ wieder durch die im Optimum bindende Nebenbedingung bestimmt:

$$
V^{1}\left(\Pi^{1}\left(e_{1}^{*}, e_{2}^{*}, z_{t+1}\right)\right)=V^{1}\left(\Pi^{i}\left(a_{t+2}\right)\right) \delta_{1} \quad(t=0,2,4, \ldots) .
$$

Die Bedingungen (5.11) und (5.12) bilden ein System rekursiver Gleichungen, die das Gleichgewicht implizit im Sinne temporärer Reaktionsfunktionen für alle Perioden $t=0,1,2, \ldots$ beschreiben.

Bei einem endlichen Zeithorizont würde solch ein System auch rekursiv und in Verbindung mit einer Bedingung, die an die letzte Periode anknüpft, gelöst. Der unendliche Zeithorizont unseres Modells läßt dies aber nicht zu. Eine explizite Lösung und damit die Bestimmung eines teilspielperfekten Gleichgewichts wird in unserem Fall erst durch die Stationaritätseigenschaft der Zeitpräferenzordnung ermöglicht. Aufgrund dessen, daß die Indifferenz zwischen zwei Periodennutzen in verschiedenen Zeitpunkten nur von der Zeitdifferenz zwischen den Perioden und nicht von den absoluten Realisierungszeitpunkten abhängt, sind die Optimierungsprobleme beider Regierungen für alle Perioden, in denen sie jeweils ein Angebot abgeben sollen, identisch. Folglich sind die optimalen Ausgleichszahlungen $z_{t}$ in Angeboten der verhandlungsbeginnenden Regierung 1 alle gleich, so daß gilt

$$
z_{0}=z_{2}=z_{4}=\ldots=: z^{1}
$$

und entsprechend gilt für Regierung 2,

$$
z_{1}=z_{3}=z_{5}=\ldots=: z^{2}
$$

Damit und mit (5.10) - (5.13) wird ein teilspielperfektes Gleichgewicht somit periodenunabhängig vollständig durch

$$
V^{1}\left(\Pi^{1}\left(e_{1}^{*}, e_{2}^{*}, z^{2}\right)\right)=V^{1}\left(\Pi^{1}\left(e_{1}^{*}, e_{2}^{*}, z^{1}\right)\right) \delta_{1}
$$




$$
V^{2}\left(\Pi^{2}\left(e_{1}^{*}, e_{2}^{*}, z^{1}\right)\right)=V^{2}\left(\Pi^{2}\left(e_{1}^{*}, e_{2}^{*}, z^{2}\right)\right) \delta_{2}
$$

charakterisiert. Die Stetigkeit der Nutzenfunktionen in z sowie ihre Definition auf einem beschränkten und abgeschlossenen Intervall sichern, daß die aus den in (5.14), (5.15) implizit bestimmten Reaktionsfunktionen $\mathbf{R}^{1}\left(\mathrm{z}^{2}\right)$ und $\mathbf{R}^{2}\left(\mathrm{z}^{1}\right)$ zusammengesetzte Funktion $\mathbf{R}^{1} \circ \mathbf{R}^{2}: \mathbf{R} \rightarrow \mathbf{R}$ mindestens einen Fixpunkt besitzt und somit Ausgleichszahlungen $\mathrm{z}^{1 *}$ und $\mathrm{z}^{2 *}$ existieren, die diese Gleichungen erfüllen. Dadurch wird ein zeitlich invariantes Angebot $\mathbf{a}^{1 *}$,

$$
a^{1 *}:=\left(e_{1}^{*}, e_{2}^{*}, z^{1 *}\right)
$$

definiert, das die verhandlungsbeginnende Regierung 1 machen würde, solange der Verhandlungsproze $B$ andauert. Ebenso wird ein zeitlich invariantes Angebot a ${ }^{2 *}$,

$$
a^{2 *}:=\left(e_{1}^{*}, e_{2}^{*}, z^{2 *}\right)
$$

definiert, das die zunächst reagierende Regierung 2 abgeben würde, solange der Verhandlungsprozeß andauert.

Analog zu dem Problem, in dem die Regierung 1 die Verhandlungen beginnt, läßt sich das Problem für einen Beginn durch Regierung 2 beschreiben. Die Stationaritätseigenschaft der Zeitpräferenzordnung kommt auch hier wieder vereinfachend zum Tragen. Die Optimierungsprobleme der beiden Regierungen sind durch die Stationarität der Zeitpräferenzen in allen Perioden, in denen diese ein Angebot abgeben, identisch. Folglich wird Regierung 2, wenn sie die Verhandlungen beginnt, das Angebot a ${ }^{2 *}$ abgeben.

Die Gleichgewichtsstrategien $F^{*}=\left\{F^{t *}\right\}_{t}=0,1, \ldots$ von Regierung 1 und $G^{*}=\left\{G^{t *}\right\}_{t=0,1, \ldots}$ von Regierung 2, die das teilspielperfekte Gleichgewicht vollständig beschreiben, enthalten somit nur Angebote vom Typ $a^{1 *}$ bzw. $a^{2 *}$. Sie enthalten außerdem die Regel, alle Angebote, die einen geringeren Nutzen als die entsprechenden eigenen Gleichgewichtsangebote in 
der nächsten möglichen Verhandlungsrunde stiften, abzulehnen und nur die anzunehmen, die einen äquivalenten oder höheren Nutzen ergeben.

Da sich die auf ein Angebot $a^{1 *}$ bzw. $a^{2 *}$ reagierende Regierung im Falle einer Ablehnung in der nächsten Verhandlungsrunde und allen weiteren Verhandlungsrunden nicht verbessern kann, wird eine rational handelnde Regierung 2 in der Periode $t=0$ einem Angebot $a^{1 *}$ von Regierung 1 sofort zustimmen, wenn diese die Verhandlungen beginnt. Entsprechendes gilt bei einem Angebot $a^{2 *}$ von Regierung 2 in Periode $t=0$.

Die Gleichgewichtsangebote $a^{1 *}$ und $a^{2 *}$ implizieren gemäß (5.9) in Verbindung mit (4.5) - (4.7) eine gesamtkostenminimale Umweltallokation $\left(e_{1}^{*}, e_{2}{ }^{*}\right)$, wobei der maximale aggregierte Effizienzgewinn $n^{*}$ erreicht wird und damit im Gleichgewicht

$$
\begin{aligned}
\Pi^{*}\left(e_{1}^{*}, e_{2}^{*}\right) & =\Pi^{1}\left(a^{1 *}\right)+\Pi^{2}\left(a^{1 *}\right) \\
& =\Pi^{1}\left(a^{2 *}\right)+\Pi^{2}\left(a^{2 *}\right)
\end{aligned}
$$

gilt. Somit werden in einem teilspielperfekten Gleichgewicht die internationalen externen Effekte mit dem vereinbarten Abkommen vollkommen internalisiert. Die in diesem Modell vorgegebenen Rahmenbedingungen üben somit keinen negativen Einfluß aus, sondern begünstigen eine effiziente Allokation der Umweltressourcen. Ihre Auswirkungen werden nur in der Verteilung des Effizienzgewinns sichtbar.

Die Gleichgewichtsbedingungen (5.14) und (5.15) sind ähnlich wie die Bedingung (4.15) des statisch-axiomatischen Verhandlungsmodells als eine Teilungsregel zu verstehen, die die Aufteilung dieses maximalen Effizienzgewinns $\pi^{*}$ bestimmen. Dabei wird deutlich, daß bei gegebenen Zeitpräferenzen mit $0<\delta_{1}, \delta_{2}<1$ diejenige Regierung einen Vorteil besitzt, die in der Stackelberg-Unabhängigkeitsposition ist und anbieten darf. Es erhält dadurch diejenige Regierung, die die Verhandlungen initiiert und ein erstes Angebot abgibt, einen größeren Anteil an den zukünftigen Effizienzgewinnen $\pi^{*}$. Beispielsweise in (5.14) muß, für $0<\delta_{1}<1$, $\Pi^{1}\left(\mathrm{a}^{1 *}\right)>\Pi^{1}\left(\mathrm{a}^{2 *}\right)$ gelten, damit die Gleichgewichtsbedingung erfüllt ist. 
Die Differenz zwischen beiden Periodennutzen steigt, je niedriger der Diskontfaktor $\delta_{1}$ ist. Entsprechendes gilt für (5.15). ${ }^{41}$

Diese Zweiteilung der notwendigen Bedingungen des teilspielperfekten Gleichgewichts in eine Effizienzbedingung und eine Aufteilungsbedingung hat entsprechende Konsequenzen bei der Untersuchung von Auswirkungen exogener Einflußfaktoren. Die Ausgangsbedingungen in den beiden Umweltregionen in Form der Entsorgungskosten- und Schadensfunktionen sowie der spezifischen Eigenschaften des Umweltsystems bestimmen ausschließlich die Höhe des Effizienzgewinns $\Pi^{*}$. In diesem Zusammenhang wurde schon in Abschnitt 4.1. der Einfluß von veränderten Assimilations- und Verteilungskräften des Umweltsystems auf die Höhe dieses Gewinns herausgearbeitet. Dagegen haben die potentiellen individuellen Kosten der Nichteinigung, die durch den Ablauf des Verhandlungsprozesses und die individuellen Zeitpräferenzen der beiden Regierungen beeinflußt werden, keine Auswirkungen auf die Gewinnhöhe $\Pi^{*} .{ }^{51}$

Die Bestimmungsgrößen des Umweltsystems haben wiederum keine Bedeutung für die Verteilung des Effizienzgewinns, d.h. es ist für das in einem teilspielperfekten Gleichgewicht erreichbare relative Nutzenniveau jeder Regierung unbedeutend, in welcher Umweltregion die einzelnen Anteile des Effizienzgewinns ihren Ursprung haben. Nur die potentiellen individuellen Kosten der Nichteinigung sind dafür ausschlaggebend. Die im nächsten Abschnitt folgende komparativ-statische Analyse hat somit nur noch die Aufgabe, die exogenen Einflüsse auf diese Kosten aufzuzeigen.

4) In einem teilspielperfekten Gleichgewicht ohne Ausgleichszahlungen, d.h. mit der Nebenbedingung $z=0$, würden die Bedingungen (5.14) und (5.15) auch die Umweltallokation bestimmen. In diesem Fall fordern die notwendigen Bedingungen (5.5) und (5.6) des Optimierungsproblems nur, daß die L'mweltallokation paretooptimal sein muß. Die genaue Bestimmung der Allokation und damit die Aufteilung des Effizienzgewinns erfolgt durch die Bedingungen (5.14) und (5.15).

5) Es sei aber an dieser Stelle schon darauf hingewiesen, daß dieses nur unter "idealen" Rahmenbedingungen gilt, d.h. die beiden Regierungen unter vollkommener und vollständiger Information agieren. 
Abschließend sollen die Eigenschaften des teilspielperfekten Gleichgewichts an einem einfachen Beispiel näher illustriert und mit den Ergebnissen des Nash-Modells aus Kapitel 4 verglichen werden. Dabei gehen wir davon aus, daß die beiden Regierungen vollkommen risikoneutral sind und sich ihre Zeitpräferenzen einfach in Form des Gegenwartswerts aller zukünftigen Umweltkostenvorteile darstellen lassen. Ihre Zielfunktion aus (5.3) reduziert sich damit auf

$$
\mathrm{U}^{\mathrm{i}}\left(\mathrm{a}_{\mathrm{t}}, \mathrm{t}\right)=\Pi^{\mathrm{i}}\left(\mathrm{a}_{\mathrm{t}}\right) \frac{\delta_{\mathrm{i}}^{\mathrm{t}}}{1-\delta_{\mathrm{i}}}
$$

Mit dieser Annahme reduzieren sich die Gleichgewichtsbedingungen aus (5.14), (5.15) unter Berücksichtigung von (5.16), (5.17) auf

$$
\begin{aligned}
& \Pi^{1}\left(\mathrm{a}^{2 *}\right)=\Pi^{1}\left(\mathrm{a}^{1 *}\right) \delta_{1}, \\
& \Pi^{2}\left(\mathrm{a}^{\prime *}\right)=\Pi^{2}\left(\mathrm{a}^{2 *}\right) \delta_{2^{2}} .
\end{aligned}
$$

Bezieht man die Effizienzbedingung (5.18) in die beiden Gleichungen ein, dann werden dadurch die Umweltkostenvorteile pro Periode der beiden Regierungen als konstanter Anteil am maximalen Effizienzgewinn $\Pi^{*}$ bestimmt. Beginnt Regierung 1 die Verhandlungen, ergibt sich die Aufteilung

$$
\left(\Pi^{1}\left(\mathrm{a}^{1 *}\right), \Pi^{2}\left(\mathrm{a}^{1^{*}}\right)\right)=\left(\frac{1-\delta_{2}}{1-\delta_{1} \delta_{2}} \Pi^{*}, \frac{\left(1-\delta_{1}\right) \delta_{2}}{1-\delta_{1} \delta_{2}} \Pi^{*}\right) .
$$

Beginnt dagegen Regierung 2, führt das zur Aufteilung

$$
\left(\Pi^{1}\left(\mathrm{a}^{2}\right), \Pi^{2}\left(\mathrm{a}^{2 *}\right)\right)=\left(\frac{\left(1-\delta_{2}\right) \delta_{1}}{1-\delta_{1} \delta_{2}} \Pi^{*}, \frac{1-\delta_{1}}{1-\delta_{1} \delta_{2}} \Pi^{*}\right) .
$$




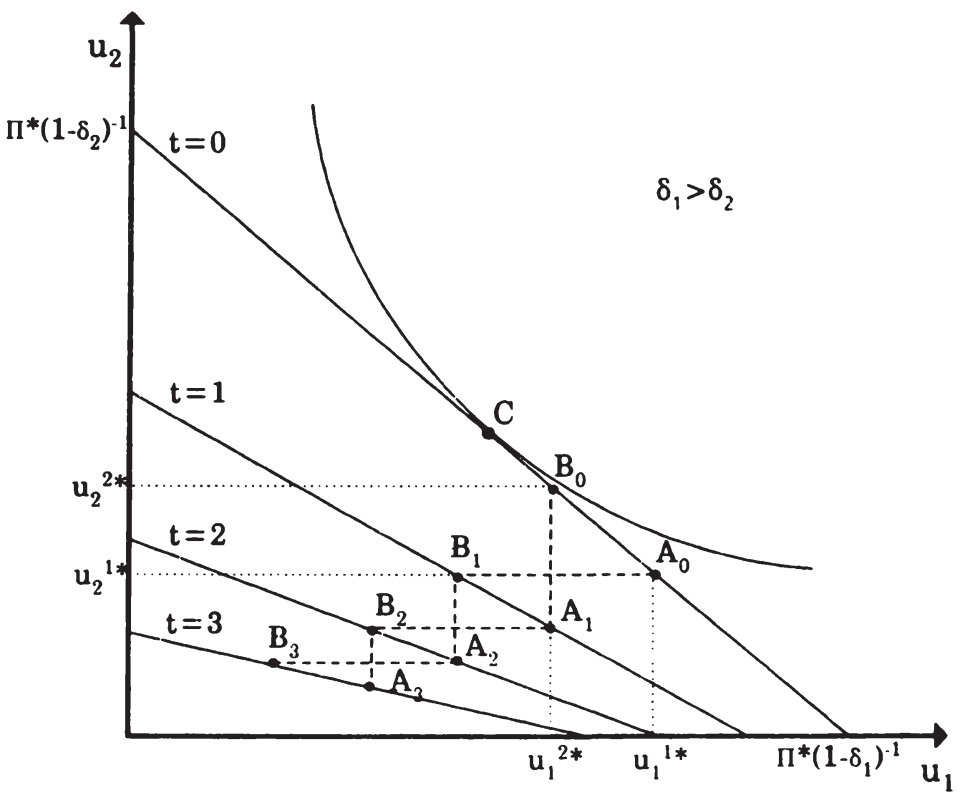

Abbildung 5.1

Abbildung 5.1 zeigt für dieses Beispiel graphisch die Nutzenverteilung in einem teilspielperfekten Gleichgewicht bei den Aufteilungen gemäß (5.20) und (5.21). Die geometrischen Orte der jeweils in einer Verhandlungsrunde $t$ noch erreichbaren Nutzenkombinationen gewinnt man, indem man die Nutzenfunktionen in (5.19) nach $\Pi^{1}$ und $\Pi^{2}$ auflöst und in die Effizienzbedingung (4.7) einsetzt:

$$
u_{2}=\frac{\delta_{2}^{t}}{1-\delta_{2}}\left[I *-\frac{\left(1-\delta_{1}\right) \delta_{2}^{t}}{\left(1-\delta_{2}\right) \delta_{1}^{t}} u_{1}\right.
$$


Beginnt Regierung 1, und man einigt sich sofort in der ersten Runde, ergibt sich mit Hilfe von (5.19) und (5.20) die Aufteilung

$$
\left(u_{1}^{1_{*}^{*}}, u_{2}^{1_{*}^{*}}\right)=\left(\frac{1-\delta_{2}}{\left(1-\delta_{1} \delta_{2}\right)\left(1-\delta_{1}\right)} \Pi^{*}, \frac{\left(1-\delta_{1}\right) \delta_{2}}{\left(1-\delta_{1} \delta_{2}\right)\left(1-\delta_{2}\right)} \Pi^{*}\right) .
$$

Diese entspricht Punkt $A_{0}$ in der Abbildung 5.1. Beginnt Regierung 2, ist es die Kombination

$$
\left(u_{1}^{2 *}, u_{2}^{2 *}\right)=\left(\frac{\left(1-\delta_{2}\right) \delta_{1}}{\left(1-\delta_{1} \delta_{2}\right)\left(1-\delta_{1}\right)} \Pi^{*}, \frac{1-\delta_{1}}{\left(1-\delta_{1} \delta_{2}\right)\left(1-\delta_{2}\right)} \Pi^{*}\right),
$$

dargestellt durch den Punkt $B_{0}$. Die von den Punkten $A_{0}$ und $B_{0}$ ausgehenden gestrichelten Linien deuten die Nutzenschranken der jeweils reagierenden Regierung zwischen Annahme und Ablehnung an, denen die anbietende Regierung bei ihrer Angebotswahl Rechnung tragen muß. Folgt man ihnen, dann beschreibt die Folge der Punkte $A_{0}, B_{1}, A_{2}, B_{3}, \ldots$ die teilspielperfekten Nutzenaufteilungen, wenn Regierung 1 in Periode $t=0$ beginnt und entsprechend die Folge der Punkte $B_{0}, A_{1}, B_{2}, A_{3}, \ldots$ den Fall, wenn Regierung 2 beginnt.

Mit dem Beispiel können wir einen direkten Vergleich zwischen der statisch-axiomatischen und der dynamisch-strategischen Verhandlungslösung ziehen, wenn wir davon ausgehen, daß die Menge der bei einer Einigung in Periode $\mathrm{t}=0$ erreichbaren Nutzenkombinationen identisch zu der Menge $W$ aus dem statischen Verhandlungsmodell ist. Dazu muß mit (5.19) gelten

$$
W^{i}(\Pi(a))=U^{i}(a, 0)=\frac{\Pi^{i}(a)}{1-\delta_{i}}
$$

Dieser Fall impliziert mit (4.8) auch $W^{i}(0)=\underline{W}_{i}=0$. Die Effizienzregel (4.16) impliziert $\Pi^{*} \equiv \Pi^{1}+\Pi^{2}$ und fordert zusammen mit der Aufteilungsregel (4.15) 


$$
\frac{Y}{1-Y}=\frac{\Pi^{1}(a)}{\Pi^{*}-\Pi^{1}(a)}
$$

Löst man die Gleichungen nach $\Pi^{1}$ und berücksichtigt daneben, daß $\Pi^{*} \equiv \Pi^{1}+\Pi^{2}$ gilt, dann ergibt sich die Aufteilung

$$
\left(\Pi^{1}(a), \Pi^{2}(a)\right)=\left(y \Pi^{*},(1-y) \Pi^{*}\right)
$$

Diese Aufteilung für das statisch-axiomatische Modell, verglichen mit den Aufteilungen (5.20) und (5.21) für das dynamisch-strategische Modell, läßt den Einfluß der zusätzlichen Information erkennen, die in der expliziten Beschreibung des Verhandlungsprozesses und den individuellen potentiellen Kosten der Nichteinigung des dynamischen Verhandlungsmodells enthalten sind. Wir können nun den Parameter y als Maß für die Verhandlungsmacht von Regierung 1 aus dem Modell heraus erklären. Beginnt Regierung 1 die Verhandlungen, ist

$$
Y^{1 *}=\frac{1-\delta_{2}}{1-\delta_{1} \delta_{2}}
$$

das Maß ihrer Verhandlungsmacht in dieser Situation. Beginnt dagegen Regierung 2, reduziert sich dieses Maß für Regierung 1 auf

$$
Y^{2 *}=\frac{\left(1-\delta_{2}\right) \delta_{1}}{1-\delta_{1} \delta_{2}}
$$

Im Fall der ursprünglichen Nash-Verhandlungslösung, d.h. endogener Bestimmung der Aufteilung durch das Symmetrie-Axiom und damit $Y=1 / 2$, teilen sich beide Regierungen gemä $B(5.25)$ den Effizienzgewinn $\Pi^{*}$ zu gleichen Teilen. Dazu sei angemerkt, daß diese Aufteilung unabhängig von der Höhe der Diskontfaktoren $\delta_{1}$ und $\delta_{2}$ ist. Diese spezielle Lösung ist in Abbildung 5.1 als Tangentialpunkt einer gleichseitigen Hyperbel an den Rand der Menge der Nutzenaufteilungen (Punkt C) dargestellt. 


\subsection{Die Auswirkungen von Veränderungen der Verhandlungs- bedingungen auf das Gleichgewicht}

Die Verteilung der Effizienzgewinne im Gleichgewicht und damit das von den Regierungen jeweils erreichbare Nutzenniveau hängen bei einem gegebenen maximalen Effizienzgewinn $\Pi^{*}$ von dem vorgegebenen Ablauf des Verhandlungsprozesses und den individuellen Zeitpräferenzen der beiden Regierungen ab. Betrachtet man die Ablaufstruktur des Verhandlungsprozesses mit alternierenden Angeboten zunächst als ein unveränderbares Merkmal des Modells, dann verbleiben die exogenen Parameter der individuellen Zeitpräferenzen und der zeitliche Abstand zwischen den einzelnen Verhandlungsrunden als exogene Größen, von denen es wichtig ist zu wissen, wie diese die endogenen Größen des Modells im Gleichgewicht beeinflussen.

Ausgangspunkt der komparativ-statischen Analyse sind die von diesen Parametern abhängigen Gleichgewichtsbedingungen in (5.14) und (5.15). Da eine Änderung dieser Größen d.h. der marginalen Diskontraten $r_{1}$ und $r_{2}$, der Periodenlänge $\tau$ und der individuellen Risikopräferenz, keinen Einfluß auf die Allokation und damit auf die Höhe des Effizienzgewinns $\Pi^{*}$ hat, sondern nur auf dessen Verteilung, vereinfacht sich die Analyse.

Gemäß (5.18) ergänzen sich die Effizienzgewinnanteile der beiden Regierungen im Gleichgewicht $z u n^{*}$. Somit ist es für diese Untersuchung ausreichend, die Veränderungen des Gewinnanteils in der StackelbergUnabhängigkeitsposition bzw. Abhängigkeitsposition für nur eine Regierung darzustellen. Wir betrachten zu diesem Zweck ausschließlich die Effizienzgewinnanteile von Regierung 1, d.h. die Umweltkostenvorteile $\Pi^{1}\left(a^{1 *}\right)$ und $\Pi^{1}\left(a^{2 *}\right)$. Zur Vereinfachung von deren Schreibweise sei im folgenden der Wert des Gewinnanteils in der Unabhängigkeitsposition kurz mit $\mathrm{n}^{11}$ bezeichnet, d.h.

$$
\Pi^{11}:=\Pi^{1}\left(\mathrm{a}^{1 *}\right)
$$


und entsprechend der in der Abhängigkeitsposition mit $\mathrm{n}^{12}$, d.h.

$$
\Pi^{12}:=\Pi^{1}\left(a^{2 *}\right)
$$

Gemäß (5.18) können wir dann die Werte der entsprechenden Anteile von Regierung 2 durch $n^{*}-n^{11}$ und $n^{*}-n^{12}$ beschreiben, $d . h$.

$$
\begin{aligned}
& \Pi^{*}-\Pi^{11}:=\Pi^{2}\left(a^{1 *}\right), \\
& n^{*}-\pi^{12}:=\Pi^{\prime \prime}\left(a^{2 *}\right),
\end{aligned}
$$

Danach lauten die Gleichgewichtsbedingungen (5.14) und (5.15)

$$
\begin{aligned}
& V^{i}\left(n^{12}\right)=V^{1}\left(n^{11}\right) e^{-r_{1} t}, \\
& V^{2}\left(n^{*}-n^{i 1}\right)=V^{2}\left(\pi^{*}-n^{12}\right) e^{-r_{2} t},
\end{aligned}
$$

wobei noch $\delta_{1}$ sowie $\delta_{2}$ gemäß $(5.2)$ durch $e^{-r_{1} \tau}$ bzw. $e^{-r_{2} \tau}$ ersetzt sind. Durch totale Differentiation bezüglich $n^{11}$ und $n^{12}$ sowie $r_{1}, r_{2}$ und $\tau$, sowie Zurücksubstituieren von $\delta_{1}$ und $\delta_{2}$, erhält man folgendes Gleichungssystem:

$$
\begin{aligned}
& {\left[\begin{array}{ll}
-V_{n}^{1}\left(\pi^{11}\right) \delta_{1} & V_{n}^{1}\left(\pi^{12}\right) \\
-V_{n}^{2}\left(\pi^{*}-\pi^{11}\right) & V_{n}^{2}\left(\pi^{*}-\pi^{12}\right) \delta_{2}
\end{array}\right]\left[\begin{array}{l}
d \pi^{11} \\
d \pi^{12}
\end{array}\right]} \\
& =\left[\begin{array}{lcc}
-\tau V^{1}\left(\pi^{11}\right) \delta_{1} & 0 & -r_{1} V^{1}\left(\pi^{11}\right) \delta_{1} \\
0 & -\tau V^{2}\left(\pi^{*}-\pi^{12}\right) \delta_{2} & -r_{2} V^{2}\left(\pi^{*}-\pi^{12}\right) \delta_{2}
\end{array}\right]\left[\begin{array}{l}
d r_{1} \\
d r_{2} \\
d r
\end{array}\right]
\end{aligned}
$$

Da mit (5.28) $\pi^{11}>\pi^{12}$ ist, impliziert die Konkavität von $V^{1}$ und $V^{2}$ 


$$
\begin{gathered}
V_{n}^{1}\left(\pi^{12}\right) \geq V_{n}^{1}\left(n^{11}\right), \\
V_{n}^{2}\left(n^{*}-n^{11}\right) \geq V_{n}^{2}\left(n^{*}-\pi^{12}\right) .
\end{gathered}
$$

Für die Systemdeterminante $\Delta$ gilt folglich

$$
\Delta=V_{\pi^{1}}^{1}\left(\pi^{12}\right) V_{n}^{2}\left(\pi^{*}-\pi^{11}\right)-V_{n}^{1}\left(\pi^{11}\right) V_{n}^{2}\left(\pi^{*}-\pi^{12}\right) \delta_{1} \delta_{2}>0 .
$$

\subsubsection{Einfluß der Zeitpräferenzen}

Eine erste wichtige Einflußgröße stellen die marginalen Diskontraten $\mathbf{r}_{1}$ und $r_{2}$ dar, die als individuelle Bestimmungsgröße der Diskontfaktoren in (5.2) eingeführt wurden. Positiv-ökonomisch interpretiert sind sie ein $\mathrm{Ma} \beta$ zur Bewertung der individuellen Kosten der Nichteinigung einer Regierung. Sie bilden damit die politische "Ungeduld" ab, bis ein Abkommen mit einem bestimmten Ergebnis zustande kommt. Normativ betrachtet und komplementär zu den Kosten der Nichteinigung bewerten sie aber auch den zukünftigen Nutzenstrom, wenn schließlich ein dauerhaftes Abkommen in einer Verhandlungsperiode geschlossen wird. Je höher die marginalen Diskontraten sind, umso größer sind die Kosten der Nichteinigung und damit die "politische Ungeduld", umso geringer wird aber auch der zukünftige Nutzenstrom vom Zeitpunkt des Verhandlungsbeginns aus bewertet.

Die Gleichgewichtsbedingungen (5.14) und (5.15) machen deutlich (und der Grund dafür liegt wieder in der Stationaritätseigenschaft der Zeitpräferenzordnung), daß nicht der Nutzen aller zukünftigen Umweltkostenvorteile einen Einfluß auf das Verhandlungsergebnis hat, sondern nur die Kosten der Nichteinigung jeder Regierung von einer Verhandlungsrunde zur nächsten. Wenn wir die marginalen Diskontraten variieren, messen wir somit vornehmlich den Einfluß von Veränderungen der "politischen Ungeduld". 
Auf welche Weise die Diskontraten $r_{1}$ und $r_{2}$ die Verteilung der Effizienzgewinne beeinflussen, läßt sich mit Hilfe der Cramerschen Regel aus dem Gleichungssystem bestimmen. Es gilt

$$
\frac{d \pi^{11}}{d r_{1}}=-\frac{\tau \delta_{1} \delta_{2} V^{1}\left(\pi^{11}\right) V_{n}^{2}\left(\pi^{*}-\pi^{12}\right)}{\Delta}<0,
$$

$$
\frac{d n^{12}}{d r_{1}}=-\frac{\delta_{1} V^{1}\left(n^{11}\right) V_{n}^{2}\left(n^{*}-n^{11}\right)}{\Delta}<0
$$

$$
\frac{d n^{11}}{d r_{2}}=\frac{w_{2} V_{n}^{1}\left(n^{12}\right) V^{2}\left(\pi^{*}-\pi^{12}\right)}{\Delta}>0
$$

$$
\frac{d \pi^{12}}{d r_{2}}=\frac{\tau \delta_{1} \delta_{2} V_{n^{1}}^{1}\left(\pi^{11}\right) V^{2}\left(\pi^{*}-\pi^{12}\right)}{\Delta}>0
$$

Gemäß diesen Multiplikatoren erhält Regierung 1 einen geringeren Anteil am Effizienzgewinn, wenn sich ihre eigene Zeitpräferenz mit der Diskontrate $r_{1}$ erhöht. Dagegen erhöht sich ihr Anteil, wenn die Diskontrate $r_{2}$ der ausländischen Regierung 2 steigt. Damit wird deutlich, daß sich mit steigenden inländischen Diskontraten und sinkenden ausländischen Diskontraten die Kosten der Nichteinigung der inländischen Regierung erhöhen, d.h. deren Gewinnanteil sinkt.

Desweiteren läßt sich aus einem Vergleich von (5.30) mit (5.31) sowie (5.32) mit (5.33) eine Aussage darüber machen, wie sich bei einer Änderung der Diskontraten der relative Vorteil, den die StackelbergUnabhängigkeitsposition gegenüber der Abhängigkeitsposition gewährt, verändert. Unter Berücksichtigung von (5.29) und $\pi^{11}>\pi^{12}$ gilt

$$
\frac{d n^{12}}{d r_{1}}<\frac{d \pi^{11}}{d r_{1}}<0,
$$




$$
\frac{d \pi^{11}}{d r_{2}}>\frac{d \pi^{12}}{d r_{2}}>0 .
$$

Steigt die Diskontrate $r_{1}$, dann ist der Rückgang des Umweltkostenvorteils geringer, wenn Regierung 1 selbst die Verhandlungen beginnt. Bei einem Anstieg von $r_{2}$ ist der Anstieg des Umweltkostenvorteils größer, wenn Regierung 1 selbst beginnt. Folglich steigt mit einer Erhöhung der Diskontraten die Attraktivität, die Verhandlungen zu beginnen.

Interessant im Hinblick auf einen Vergleich zwischen dem statischaxiomatischen Modell ohne Transaktionskosten und dem dynamischstrategischen Modell sind die Grenzfälle, wenn eine oder beide Regierungen eine nur sehr geringe Zeitpräferenz besitzen. Nimmt die Diskontrate eines Landes annähernd den Wert Null an, entstehen einer Regierung keine Kosten der Nichteinigung.

Dazu betrachten wir die Gleichgewichtsbedingungen in (5.28). Besitzt beispielsweise die Diskontrate $r_{1}$ von Regierung 1 annähernd den Wert Null, während für die Diskontrate von Regierung 2 gilt $r_{2}>0$, werden diese Bedingungen nur von $n^{11}=n^{12}=n^{*}$ erfüllt. Regierung 1 erhält somit den gesamten Effizienzgewinn $n^{*}$. Vollkommen anders sieht die Situation aus, wenn beide Regierungen keine Zeitpräferenz haben. Gilt im Grenzfall für beide Diskontraten $r_{1}=r_{2}=0$, dann bleibt die Lösung unbestimmt. ${ }^{6}$ Jede Gewinnaufteilung erfüllt die Gleichgewichtsbedingungen. Dieses letzte Resultat entspricht im wesentlichem dem von Kapitel 4, wonach auf einem Verhandlungsmarkt ohne Transaktionskosten jede effiziente Lösung möglich ist. Die dynamisch-strategische Lösung hat damit bestätigt, daß eine gewisse Unvollkommenheit auf Verhandlungsmärkten verbunden mit positiven potentiellen Transaktionskosten notwendig ist, damit sich ein bestimmtes Ergebnis ergibt.

6) Vergleiche zu diesem Ergebnis auch Binmore, Rubinstein und Wolinsky (1985). 


\subsubsection{Zeitlicher A bstand der Verhandlungsrunden}

Eine zweite für uns wichtige Größe ist der zeitliche Abstand $\tau$ zwischen den Verhandlungsrunden, der ebenfalls gemäß (5.2.) als Bestimmungsgröße der im Modell verwendeten Diskontfaktoren $\delta_{1}$ und $\delta_{2}$ dient. Während die Diskontraten ein relatives und individuelles $\mathrm{Ma}$ für den Einfluß von Zeit zwischen den Regierungen sind, ist die zeitliche Distanz $\tau$ im Vergleich dazu ein absolutes und für beide Regierungen gleiches Maß. Hier wird insbesondere die Frage im Vordergrund stehen, ob der Vorteil, die Verhandlungen zu beginnen sich verringert, wenn der Verhandlungsprozeß schneller ablaufen würde, d.h. wenn $\tau$ sinkt.

Verändert sich der zeitliche Abstand $\tau$ zwischen den einzelnen Verhandlungsrunden und damit die Länge der Perioden, lassen sich zunächst nur relative Aussagen treffen. Aus dem Gleichungssystem können wir wiederum bestimmen:

$$
\frac{d n^{11}}{d \tau}=\frac{\delta_{2}\left[r_{2} V^{2}\left(\pi^{*}-n^{12}\right) V_{n}^{1}\left(\pi^{12}\right)-r_{1} V^{1}\left(n^{12}\right) V_{n}^{2}\left(\pi^{*}-\pi^{12}\right) \mid\right.}{\Delta},
$$

$$
\frac{d n^{12}}{d \tau}=\frac{\delta_{1}\left[r_{2} V^{2}\left(\pi^{*}-\pi^{11}\right) V_{\pi^{1}}^{1}\left(\pi^{11}\right)-r_{1} V^{1}\left(\pi^{11}\right) V_{\pi^{2}}^{2}\left(\pi^{*}-\pi^{11}\right) \mid\right.}{\Delta} .
$$

Diese Änderungen haben kein eindeutiges Vorzeichen. Es hängt somit von der Größe der Diskontfaktoren und den Nutzenfunktionen ab, ob ein absoluter Vor- oder Nachteil für eine Regierung mit einer Veränderung des zeitlichen Abstands der Verhandlungsrunden einhergeht. Substituiert man aber gemäß (5.28) in (5.34) $\mathrm{V}^{2}\left(\mathrm{n}^{*}-\pi^{12}\right) \delta_{2}$ durch $\mathrm{V}^{2}\left(\mathrm{n}^{*}-\mathrm{n}^{11}\right)$ und in (5.35) $\delta_{1} V^{1}\left(n^{11}\right)$ durch $V^{1}\left(n^{12}\right)$ und bildet die Differenz aus beiden Größen, so zeigt sich bei Berücksichtigen von (5.29) 


$$
\begin{aligned}
& \frac{d \pi^{11}}{d \tau}-\frac{d n^{12}}{d \tau}=\frac{r_{2} V^{2}\left(n^{*}-n^{11}\right) V_{n}^{1}\left(n^{12}\right)-r_{1} \delta_{2} V^{1}\left(n^{12}\right) V_{n}^{2}\left(n^{*}-n^{12}\right)}{\Delta} \\
& -\frac{\delta_{1} r_{2} V^{2}\left(n^{*}-n^{11}\right) V_{n^{1}}^{1}\left(n^{11}\right)-r_{1} V^{1}\left(n^{12}\right) V_{n}^{2}\left(n^{*}-n^{11}\right)}{\Delta} \\
& =\frac{\left.r_{2} V^{2}\left(\pi^{*}-\pi^{11}\right)\left(V_{\pi^{1}}^{1}\left(\pi^{12}\right)-\delta_{1} V_{\pi^{1}}^{1}\left(\pi^{11}\right)\right)+r_{1} V^{1}\left(\pi^{12}\right)\left(V_{n}^{2}\left(\pi^{*}-\pi^{11}\right)-\delta_{2} V_{\pi^{2}}^{2} \pi^{*}-\pi^{12}\right)\right)}{\Delta}>0
\end{aligned}
$$

Eine Vergrößerung des zeitlichen Abstands $\tau$ zwischen den Verhandlungsrunden vergrößert den Unterschied zwischen dem Effizienzgewinnanteil, den Regierung 1 erhält, wenn diese die Unabhängigkeitsposition einnimmt, gegenüber dem in der Abhängigkeitsposition.

Je kürzer die einzelnen Verhandlungsrunden aufeinanderfolgen, umso geringer ist der Vorteil, den man aus der strategisch günstigeren Unabhängigkeitsposition ziehen kann. Anhand des oben dargestellten Beispiels mit einer risikoneutralen Zeitpräferenzordnung läßt sich zeigen, daß dieser Vorteil verschwindet, wenn die Periodenlänge sehr klein wird. Wir bestimmen dazu den Grenzwert der Effizienzgewinnanteile $\pi^{11}$ und $\pi^{12}$ für den Fall, daß $\tau$ gegen null strebt. Das Grenzwertproblem für $\pi^{11}$ wird unter Berücksichtigen von (5.20) und der Definition der Diskontfaktoren in (5.2) bestimmt durch

$$
\lim _{t \rightarrow 0} \pi^{11}=\lim _{\tau \rightarrow 0} \frac{1-e^{-\tau r_{2}}}{1-e^{-\tau\left(r_{1}+r_{2}\right)}} \pi^{*} .
$$

Das für $\mathrm{n}^{12}$ analoge Grenzwertproblem läßt sich daraus durch Einsetzen in die Gleichgewichtsbedingung in (5.19), $\pi^{12}=\pi^{11} e^{-r_{1} \tau}$, ableiten: 


$$
\lim _{\tau \rightarrow 0} \pi^{12}=\lim _{\tau \rightarrow 0} \frac{e^{-\tau r_{1}}-e^{-\tau\left(r_{1}+r_{2}\right)}}{1-e^{-\tau\left(r_{1}+r_{2}\right)}} \Pi^{*} .
$$

Beide Grenzwertprobleme können mit der Regel von de l'Hôspital gelöst werden. Entsprechende Differentation von Zähler und Nenner ergibt:

$$
\begin{aligned}
& \lim _{t \rightarrow 0} \pi^{11}=\lim _{t \rightarrow 0} \frac{r_{2} e^{-t r_{2}}}{\left(r_{1}+r_{2}\right) e^{-\tau\left(r_{1}+r_{2}\right)} n^{*}=\frac{r_{2}}{r_{1}+r_{2}} n^{*}} \\
& \lim _{\tau \rightarrow 0} \pi^{12}=\lim _{r \rightarrow 0} \frac{-r_{1} e^{-\tau r_{1}}+\left(r_{1}+r_{2}\right) e^{-\tau\left(r_{1}+r_{2}\right)}}{\left(r_{1}+r_{2}\right) e^{-\tau\left(r_{1}+r_{2}\right)}} \pi^{*}=\frac{r_{2}}{r_{1}+r_{2}} n^{*}
\end{aligned}
$$

Im Ergebnis zeigt sich, daß Regierung 1 bei einem sehr schnellen Verhandlungsablauf im teilspielperfekten Gleichgewicht, unabhängig davon ob sie die Verhandlungen beginnt oder nicht, immer den gleichen Anteil am Effizienzgewinn erhält. Dieser Grenzfall impliziert die Aufteilung

$$
\left(\Pi^{1}\left(a^{1 *}\right), \Pi^{2}\left(a^{1 *}\right)\right)=\left(\Pi^{1}\left(a^{2 *}\right), \Pi^{2}\left(a^{2 *}\right)\right)=\left(\frac{r_{2}}{r_{1}+r_{2}} \Pi^{*}, \frac{r_{1}}{r_{1}+r_{2}} \Pi^{*}\right)
$$

Diese Aufteilung ist wieder kompatibel mit der Aufteilung (5.25), der verallgemeinerten Nash-Verhandlungslösung für den Fall

$$
y^{1 *}=y^{2 *}=\frac{r_{2}}{r_{1}+r_{2}} \cdot 7
$$

Nur bei gleichen Zeitpräferenzen der beiden Regierungen in Form gleicher Diskontraten $r_{1}=r_{2}=r$ und einem unendlich schnellen Verhandlungsablauf ergibt sich eine gleiche Aufteilung des Effizienzgewinns, die unabhängig davon ist, wer die Unabhängigkeits- und wer die Abhängigkeitsposition einnimmt. Ausschließlich in diesem Fall ist das

7) Binmore (1980) hat dieses von uns analytisch abgeleitete Ergebnis graphisch gezeigt. 
Ergebnis des ursprünglichen Nash-Verhandlungsmodells kompatibel mit unserer nichtkooperativen Lösung.

Binmore, Rubinstein und Wolinsky (1985) haben über dieses Beispiel hinaus gezeigt, daß dieses Ergebnis auch im risikoaversen Fall gilt. Geht man somit von einem sehr schnellen Verhandlungsablauf aus und unterstellt den Regierungen gleiche Zeitpräferenzen, dann bestimmt der dynamisch-strategische Ansatz die ursprüngliche Nash-Lösung als einen Spezialfall.

\subsubsection{Einfluß von Risikoaversion}

Die dritte in der Zeitpräferenzordnung für das Verhandlungsergebnis bedeutungsvolle Einflußgröße ist das Maß der individuellen Risikopräferenz. Untersucht man diese im Gleichgewicht eines Verhandlungsspiels, in dem die Verhandelnden ohne Unsicherheit und mit vollständiger Information agieren, so betrachtet man zunächst nur das "strategische Risiko". Es bezieht sich darauf, da $\beta$ trotz der vorhandenen Sicherheit im Verlauf der Verhandlungen möglicherweise eine von der optimalen Strategie abweichende und unbeabsichtigte Fehlentscheidung getroffen wird. Dies wird von Selten (1975) als "trembling hand" bezeichnet. ${ }^{8)}$ Für uns erscheint in diesem Zusammenhang wichtiger, daß diese Untersuchung eine Vorhersage zuläßt, wie sich eine unterstellte Unsicherheit im Modell auswirkt. Damit greifen wir an dieser Stelle auf die nächsten Abschnitte vor, in denen Unsicherheit eingeführt wird.

8) Selten (1975) verfeinert von diesem Argument ausgehend nochmals das Konzept teilspielperfekter Nash-Gleichgewichte und formuliert ein "perfektes Gleichgewicht". Teilspielperfekte Gleichgewichte haben die Eigenschaft, daß sie die irgendwann im Spielverlauf gemachten Fehler berücksichtigen und bestimmen, wie man sich in der Zukunft trotz des Fehlers optimal verhalten soll. Eine perfekte Strategie im Sinne von Selten (1975) hat noch die zusätzliche Figenschaft, daß sie sogar die noch möglichen zukünftigen Fehler mit berücksichtigt. Myerson (1978) hat mit seinem Konzept des "proper equilibrium" dieses Konzept noch weiter pointiert. Seine prinzipielle Idee ist, daß schwerwiegende Fehler mit geringerer Wahrscheinlichkeit als andere gemacht werden und man diese Lngleichverteilung in der Gleichgewichtsstrategie berücksichtigen muß. Vergleiche dazu auch van Damme (1983, 113ff.). 
Um die Auswirkungen von Risikoaversion zu untersuchen, vergleichen wir die Umweltkostenvorteile, die Regierung 1 bzw. 2 bei vollkommen identischen Zeitpräferenzordnungen im Gleichgewicht erzielt hätten, mit einer Situation, in der Regierung 2 stärker risikoavers ist.Ausgangspunkt sei somit eine für beide Regierungen identische Nutzenfunktion $U^{i}$,

$$
\mathrm{U}^{1}\left(\mathrm{a}_{\mathbf{t}}, \mathrm{t}\right)=\mathrm{U}^{2}\left(\mathrm{a}_{\mathrm{t}}, \mathrm{t}\right):=\mathrm{V}(\mathrm{\Pi}(\mathrm{a})) \frac{\delta^{\mathrm{t}}}{1-\delta}
$$

In einem teilspielperfekten Gleichgewicht gilt damit gemäß (5.14) und (5.15)

$$
\begin{aligned}
& \mathbf{V}\left(\Pi^{\mathrm{L}}\left(\mathrm{a}^{2 *}\right)\right)=\delta \mathbf{V}\left(\Pi^{\mathrm{i}}\left(\mathbf{a}^{\mathrm{i} *}\right)\right) \\
& \mathrm{V}\left(\Pi^{2}\left(\mathrm{a}^{1 *}\right)\right)=\delta \mathrm{V}\left(\Pi^{2}\left(\mathrm{a}^{2 *}\right)\right)
\end{aligned}
$$

Nutzen wir die Effizienzbedingung (5.18) und die verkürzte Schreibweise für die Werte der Effizienzgewinnanteile, d.h. $\pi^{11}=\Pi^{1}\left(a^{1 *}\right), \pi^{22}=\Pi^{2}\left(a^{2 *}\right)$, $\Pi^{*}-\pi^{22}=\Pi^{1}\left(a^{2 *}\right)$ sowie $n^{*}-\pi^{11}=\Pi^{2}\left(a^{1 *}\right)$, dann lauten diese

$$
\begin{aligned}
& V\left(\pi^{*}-\pi^{22}\right)=\delta V\left(\pi^{11}\right), \\
& V\left(\pi^{*}-\pi^{11}\right)=\delta V\left(\pi^{22}\right) .
\end{aligned}
$$

In diesem symmetrischen Fall sind die Umweltkostenvorteile der beiden Regierungen, wenn sie jeweils die Verhandlungen beginnen würden, gleich, d.h. $\pi^{11}=\pi^{22}:=\pi^{+}$. Sie werden implizit durch die Gleichung

$$
V\left(n^{*}-n^{i}\right)=\delta V\left(n^{*}-V^{-1}\left(\delta V\left(n^{i}\right)\right)\right) \quad(i=1,2)
$$

bestimmt, die sich nach Auflösen von (5.38) nach $\mathrm{n}^{11}$ bzw. $\mathrm{n}^{22}$ und Einsetzen in (5.39) ergibt. Dabei bezeichnet $\mathrm{V}^{-1}$ die Inverse der Funktion $\mathrm{V}$.

Eine relativ stärkere Risikoaversion von Regierung 2 läßt sich nun mit einer Transformation der Funktion $\mathrm{V}$ durch eine monoton steigende, 
konkave Funktion $\mathrm{H}$ mit $\mathrm{H}(0)=0$ abbilden..$^{9}$ Dazu gelte gegenüber (5.37)

$$
\mathrm{C}^{-2}\left(\mathrm{a}_{\mathrm{t}}, \mathrm{t}\right):=\mathrm{H}[\mathrm{V}(\Pi(\mathrm{a}))] \frac{\delta^{\mathrm{t}}}{1-\delta} .
$$

Im Gleichgewicht gilt ensprechend zu (5.38) und (5.39)

$$
\begin{aligned}
& V\left(\pi^{*}-\pi^{22}\right)=\delta V\left(\pi^{11}\right), \\
& H\left[V\left(n^{*}-\pi^{11}\right)\right]=\delta H\left[V\left(\pi^{22}\right)\right] .
\end{aligned}
$$

Entsprechende Auflösung der beiden Gleichungen definiert analog zu (5.40) die Bestimmungsgleichungen für die Umweltkostenvorteile $\mathrm{n}^{11}$ und $\mathrm{n}^{22}:$

$$
\begin{aligned}
& V\left(\pi^{*}-\pi^{11}\right)=H^{-1}\left[\delta H\left[V\left(\pi^{*}-V^{-1}\left(\delta V\left(\pi^{11}\right)\right)\right)\right]\right], \\
& V\left(n^{*}-\pi^{22}\right)=\delta V\left(\pi^{*}-V^{-1}\left(H^{-1}\left[\delta H\left[V\left(\pi^{22}\right)\right]\right]\right) .\right.
\end{aligned}
$$

Die Konkavität von $\mathrm{H}$ impliziert bei $\mathrm{H}(0)=0$ zusammen mit $0<\delta<1$ auf der rechten Seite von (5.42) und (5.43), ${ }^{101} \mathrm{da} \beta$

$$
\begin{aligned}
\mathbf{H}^{-1}[\delta \mathrm{H}[\mathrm{V}(\cdot)]] & \leq \mathrm{H}^{-1}[\mathrm{H}[\delta \mathrm{V}(\cdot)]] \\
& =\delta \mathrm{V}(\cdot),
\end{aligned}
$$

wenn man den Diskontfaktor $\delta$ mit in die Funktion $\mathrm{H}$ einbezieht. Für die Gleichgewichtsbedingung (5.42) folgt damit

9) Gemäß dem Arrow-Pratt-Maß der Risikoaversion $R$ für eine Funktion $f, R=-f^{\prime \prime} / f$, verstårkt eine solche Transformation die Risikoaversion. In unserem Fall gilt

$$
\mathrm{R}=-\frac{\mathrm{H}^{\prime \prime} \mathrm{V}^{\prime}+\mathrm{H}^{\prime} \mathrm{V}^{\prime \prime}}{\mathrm{H}^{\prime} \mathrm{V}^{\prime}}=-\frac{\mathrm{H}^{\prime \prime}}{\mathrm{H}^{\prime}}-\frac{\mathrm{V}^{\prime \prime}}{\mathrm{V}^{\prime}} \geq 0 .
$$

Mit $H$ und V konkav, d.h. $H^{\prime \prime}, V^{\prime \prime}<0$ und monoton steigend, $H^{\prime}, V^{\prime}>0$, erhöht sich durch die Anwendung von $\mathrm{H}$ auf die Funktion $\mathrm{V}$ das Maß der Risikoaversion $\mathrm{R}$.

10) Für eine konkave Funktion $H$ und $0 \leq \lambda \leq 1$ gilt $H(\lambda a+(1-\lambda) b) \geq \lambda H(a)+(1-\lambda) H(b)$. Mit $b=0$ und $H(0)=0$ folgt damit $H(\lambda a) \geq \lambda H(a)$. 


$$
\begin{aligned}
V\left(\boldsymbol{n}^{*}-\boldsymbol{n}^{11}\right) & =\mathrm{H}^{-1}\left[\delta \mathrm{H}\left[\mathrm{V}\left(\boldsymbol{n}^{*}-\mathrm{V}^{-1}\left(\delta \mathrm{V}\left(\boldsymbol{\pi}^{11}\right)\right)\right)\right]\right] \\
& \leq \delta \mathrm{V}\left(\boldsymbol{n}^{*}-\mathrm{V}^{-1}\left(\delta \mathrm{V}\left(\boldsymbol{n}^{11}\right)\right)\right)
\end{aligned}
$$

Dieser Zusammenhang läßt sich direkt mit der Gleichgewichtsbedingung (5.40) für identische Präferenzen vergleichen. Da die Terme alle monoton fallend in $\pi^{11}$ sind, muß somit $\pi^{11}$ in (5.42) größer als $\pi^{11}=\pi^{+}$in (5.40) sein. Es gilt analog dazu für (5.43) unter Berücksichtigung von (5.44)

$$
\begin{aligned}
V\left(n^{*}-n^{22}\right) & =\delta V\left(n^{*}-V^{-1}\left(H^{-1}\left[\delta H\left[V\left(n^{22}\right)\right]\right]\right)\right) \\
& \geq \delta V\left(n^{*}-V^{-1}\left(\delta V\left(n^{22}\right)\right)\right)
\end{aligned}
$$

Durch das umgekehrte Ungleichheitszeichen ist somit $\pi^{22}$ in (5.43) kleiner als $\pi^{22}=\pi^{+}$in (5.40).

Damit ist gezeigt, daß sich eine relativ stärkere Risikoaversion von Regierung 2 im Vergleich zu Regierung 1 immer zugunsten von Regierung 1 auswirkt. Beginnt Regierung 1 die Verhandlungen, steigt ihr Umweltkostenvorteil gegenüber der Situation mit gleicher Risikopräferenz der beiden Regierungen; entsprechend sinkt natürlich der Umweltkostenvorteil von Regierung 2. Beginnt diese selbst die Verhandlungen, ist ihr Umweltkostenvorteil wiederum geringer gegenüber der Vergleichssituation, und der entsprechende Umweltkostenvorteil von Regierung 1 erhöht sich. ${ }^{101}$

\subsection{Die Auswirkungen von Verānderungen im Umweltsystem auf das Gleichgewicht}

In den vorausgegangenen Abschnitten dieses Kapitels haben wir vereinfachend unterstellt, daß sich das Umweltsystem unserer Modellökonomie

10) Vergleiche zu diesem Ergebnis auch Roth (1985). 
über einen unendlichen Zeitraum hinweg in einem stationären und stabilen Gleichgewichtszustand befindet. Ebenso bleiben Entsorgungskostenund Schadensfunktionen in diesem Zeitraum unverändert.

Die Vorteile solcher "stabilen" Ausgangsbedingungen vor einem unendlichen Zeithorizont sind, daß wir damit zum einen dem Verhandlungsprozeß keine zeitlichen Restriktionen auferlegen, die möglicherweise das Verhandlungsergebnis verzerren würden. Zum zweiten gibt man damit für jede mögliche Verhandlungsrunde die gleichen Startbedingungen vor und vereinfacht so die analytische Darstellung des gesamten Verhandlungsproblems unter dem nichtkooperativen Gleichgewichtskonzept.

Diese vereinfachte Vorgehensweise ist natürlich nicht frei von unerwünschten Nebeneffekten, die das Modell nur zur Beschreibung spezieller Situationen geeignet erscheinen lassen. Solche Effekte können aber weitgehend durch eine Erweiterung der Modellannahmen beseitigt werden.

Einer der kritischen Punkte des Modells ist, daß durch den unendlichen Zeithorizont des Modells die Zeitpräferenzen der Regierungen nur die Nutzenverluste widerspiegeln, die durch die zeitverzögerte Realisierung der entsprechenden Effizienzgewinnanteile entstehen. Im Modell läßt sich dieses Phänomen mit einer Veränderung des Bezugszeitpunkts für eine nutzenmäßige Bewertung der zukünftigen Effizienzgewinnanteile verdeutlichen. Würden wir als Bezugszeitpunkt anstatt des Verhandlungsbeginns den jeweiligen Einigungszeitpunkt wählen, wäre der Nutzen der Effizienzgewinnanteile im Zeitverlauf eine konstante Größe. Faktisch wird dadurch das Verhandlungsproblem auf einen Fall reduziert, in dem ein bestimmter Betrag oder ein Gut mit einem bestimmten Wert für jede Regierung zwischen den beiden aufgeteilt werden soll. ${ }^{121}$

12) Somit läßt sich das ursprüngliche Modell praktisch auf alle die Fälle übertragen, in denen in einem bestimmten, endlichen Zeitraum, dessen Anfangszeitpunkt beliebig wählbar ist, ein bestimmter Effizienzgewinn realisiert wird und zwischen den beteiligten Ländern aufgeteilt werden muß. Dazu gehören Projekte grenzüberschreitender Zusammenarbeit mit einer bestimmten erwartungsgemäßen Lebensdauer, wie beispielsweise gemeinsame Klärwerke, bei denen u.a. im Rahmen der Kosten-Nutzen-Analyse und den Projektverhandlungen der Schlüssel festzulegen ist, nach welchem die Projektkosten auf die beteiligten Länder umzulegen sind. 
Ein zweiter kritischer Punkt des Modells ist, daß die Ausgangsbedingungen über den vorgegebenen unendlichen Zeitraum stabil sind. Dies schränkt die Aussagekraft des Modells im Hinblick auf die Erklärung realer Phänomene ein, da sich in der Realität solche Ausgangsbedingungen im Zeitverlauf sicherlich verändern.

Solche Veränderungen hängen, wie schon in der Umweltliteratur mehrfach aufgezeigt, im wesentlichen von den aktuellen Emissionsbelastungen und somit von den bestehenden Emissionsbeschränkungen ab. Je schärfer solche gesetzlichen Beschränkungen sind, umso eher wird ein reales Umweltsystem in dem von uns für das Modell unterstellten stationären Gleichgewichtszustand zwischen Immissionserhöhungen durch Emissionszugang und Immissionsabnahme durch Assimilation sein.

Je weniger restriktiv die Umweltregulierung für ein intensiv genutztes Umweltsystems ist, umso größer ist die Gefahr, daß ein solches System aus dem Gleichgewicht gerät und "umkippt", d.h. sich dessen Assimilationskräfte durch eine Überbelastung drastisch verringern. Somit wird man in diesem Zusammenhang nur über bestimmte endliche Zeiträume hinweg oder nur innerhalb gewisser Bandbreiten von Emissionsbelastungen von "stabilen" Ausgangsbedingungen reden können.

Die Modellerweiterungen in den folgenden Unterabschnitten zielen darauf ab, diese Kritikpunkte aufzugreifen und zumindest teilweise durch entsprechende alternative Annahmen zu entkräften.

\subsubsection{Stabile Ausgangsbedingungen des Umweltsystems über endliche Zeiträume}

Treten im Zeitverlauf sprunghafte Veränderungen der Ausgangsbedingungen auf, die zeitlich und in ihrer Größe determiniert sind, kann man das Verhandlungsproblem über den unendlichen Zeitraum als eine unendliche Folge von Teilverhandlungsproblemen über jeweils endliche 
Zeiträume betrachten, in denen jeweils stabile Ausgangsbedingungen herrschen. Das teilspielperfekte Gleichgewichtskonzept erfordert zur Lösung eines solchen Systems eine rekursive Vorgehensweise, so daß bei der Auswertung der einzelnen Teilprobleme Bedingungen über die jeweilige Aufteilung des entsprechenden Effizienzgewinns in dem jeweiligen Folgeproblem angegeben werden müssen. ${ }^{131}$

Wir beschränken uns in der Darstellung auf den analytisch einfachsten Fall, in dem das internationale Umweltproblem nur noch eine bestimmte Zeit lang besteht, bzw. nur noch über einen bestimmten Zeitraum Effizienzgewinne durch eine internationale Koordination erzielt werden können. Im dynamischen Verhandlungsmodell wird dazu die Anzahl der Perioden, in denen Effizienzgewinne möglich sind, beschränkt und eine Bedingung an die nachfolgenden Perioden ohne Effizienzgewinne gestellt. $^{141}$

Wir modifizieren dazu unser dynamisches Verhandlungsmodell und nehmen an, daß nur bis einschließlich in Periode $t=T$ Effizienzgewinne realisiert werden können. Dadurch verändert sich die Nutzenfunktion $U^{i}$, die in (5.3) die Zeitpräferenzen für einen unendlichen Zeithorizont beschreibt. Sie wird nun definiert durch

$$
U^{i}\left(a_{t}, t, T\right):=V^{i}\left(\Pi^{i}\left(a_{t}\right)\right) \frac{1-\delta_{i}^{T+1}}{1-\delta_{i}}-V^{i}\left(\Pi^{i}\left(a_{t}\right)\right) \frac{1-\delta_{i}^{t}}{1-\delta_{i}} \quad(i=1,2)
$$

13) Dazu vergleichbare Problemstellung gibt es in der Investitionstheorie bei der Auswahl des vorteilhaftesten Investitionsprojekts aus einer Reihe von möglichen Projekten mit unterschiedlichen Laufzeiten. Dabei muß jeweils die Vorteilhaftigkeit von Anschlußprojekten mit berücksichtigt werden.

14) Beispiele dafür in der Realität sind internationale Umweltprobleme, ausgelöst durch Industrien, bei denen vorhersehbar ist, daß die Produktion innerhalb bestimmter Zeiträume eingestellt wird. Das können Grundstoffindustrien sein, deren Lagerstätten bei einem vorgegebenen Abbauprofil nach einer gewissen Zeit erschöpft sind, wie die Kaliminen im Elsaß um die Jahrhundertwende. Ein anderes Beispiel sind umweltintensive Produktionen, wie beispielsweise bestimmte Feuerungsformen bei Kohlekraftwerken, die im Zuge des technischen Fortschritts nach einer gewissen Zeit nicht mehr in der Form existieren, weil die Altanlagen durch neue ersetzt wurden. 
Gemäß der Summenformel für eine endliche geometrische Reihe stellt der erste Summand den Nutzen bei sofortiger Einigung über einem Angebot $a_{t}$ bis einschließlich zur Periode T dar. Der zweite, negative Summand mißt den Nutzenverlust bei Nichteinigung bis zur Periode t-1 einschließlich. Der Ausdruck läßt sich zusammenfassen zu

$$
\mathrm{U}^{\mathrm{i}}\left(\mathbf{a}_{\mathrm{t}}, \mathrm{t}, \mathrm{T}\right):=\mathrm{V}^{\mathrm{i}}\left(\Pi^{\mathrm{i}}\left(\mathbf{a}_{\mathrm{t}}\right)\right) \frac{\delta_{\mathrm{i}}^{\mathrm{t}}-\delta_{\mathrm{i}}^{\mathrm{T}+1}}{1-\delta_{\mathrm{i}}}
$$

Diese modifizierte Nutzenfunktion besitzt im Gegensatz zu der des ursprünglichen Modells im Aggregat nicht mehr die Stationaritätseigenschaft. Das impliziert, daß sich die Gleichgewichtsbedingungen in einem teilspielperfekten Nash-Gleichgewicht dieses dynamischen Verhandlungsmodells im Periodenverlauf verändern und somit zur Lösung des Gleichgewichtsproblems die Bedingungen für alle möglichen Verhandlungsrunden herangezogen werden müssen.

Die auf der Basis dieser Nutzenfunktionen analog zu (5.4) und (5.5) zu lösenden Optimierungprobleme beider Regierungen für alle Perioden $t=0, \ldots, T$ führen zu den (5.11) und (5.12) entsprechenden Gleichgewichtsbedingungen:

$$
\begin{aligned}
& V^{1}\left(\Pi^{1}\left(a_{t-1}^{2}+\right)\right) \frac{\delta_{1}^{t-1}-\delta_{1}^{T+1}}{1-\delta_{1}}=V^{1}\left(\Pi^{1}\left(a_{t}^{1 *}\right)\right) \frac{\delta_{1}^{t}-\delta_{1}^{T+1}}{1-\delta_{1}} \quad(t=0, \ldots, T) \\
& V^{2}\left(\Pi^{2}\left(a_{t}^{1 *}\right)\right) \frac{\delta_{2}^{t}-\delta_{2}^{T+1}}{1-\delta_{2}}=V^{2}\left(\Pi^{2}\left(a_{t+1}^{2} *\right)\right) \frac{\delta_{2}^{t+1}-\delta_{2}^{T+1}}{1-\delta_{2}} \quad(t=0, \ldots, T)
\end{aligned}
$$

wobei $\mathrm{a}_{\mathrm{t}}{ }^{*}, \mathrm{a}_{\mathrm{t}-1}{ }^{*}$ und $\mathrm{a}^{2}{ }_{\mathrm{t}+1}{ }^{*}$ die analog $\mathrm{zu}(5.16)$ und (5.17) bestimmten, aber nun zeitabhängigen Gleichgewichtsangebote der Regierung 1 bzw. 2 darstellen. Die Gleichgewichtsbedingungen lassen sich umformen zu 


$$
\begin{array}{ll}
V^{1}\left(\Pi^{1}\left(a_{t-1}^{2}\right)\right)=V^{1}\left(\Pi^{1}\left(a_{t}^{1}\right)\right) \frac{\delta_{1}^{t}-\delta_{1}^{T+1}}{\delta_{1}^{t-1}-\delta_{1}^{T+1}} & (t=0, \ldots, T) \\
V^{2}\left(\Pi^{2}\left(a_{t}^{1^{*}}\right)\right)=V^{2}\left(\Pi^{2}\left(a_{t+1}^{2}\right)\right) \frac{\delta_{2}^{t+1}-\delta_{2}^{T+1}}{\delta_{2}^{t}-\delta_{2}^{T+1}} & (t=0, \ldots, T)
\end{array}
$$

Durch die Gleichgewichtsbedingungen sind wiederum die teilspielperfekten Gleichgewichtsstrategien $F^{*}=\left\{F^{t *}\right\}_{t}=0, \ldots, T$ für Regierung 1 und $\mathrm{G}^{*}=\left\{\mathrm{G}^{\mathrm{t}}\right\}_{\mathrm{t}=0, \ldots, \mathrm{T}}$ für Regierung 2 bestimmt. Danach kommt es wieder zu einer sofortigen Einigung, da es für die in der ersten Verhandlungsrunde reagierende Regierung individuell-rational ist, dem teilspielperfekten Eröffnungsangebot $\mathrm{a}^{1}{ }_{0}{ }^{*}$ von Regierung $1 \mathrm{bzw} . \mathrm{a}^{2}{ }_{0}{ }^{*}$ von Regierung 2, die Teil der jeweiligen Gleichgewichtsstrategie sind, zuzustimmen. Diese optimalen Eröffnungsangebote und ebenso die möglichen teilspielperfekten Folgeangebote implizieren erneut eine gesamtkostenminimale Umweltallokation $\left(\mathrm{e}_{1}{ }^{*}, \mathrm{e}_{2}{ }^{*}\right)$, da die Umweltbedingungen gegenüber dem urspünglichen dynamischen Modell unverändert blieben.

Bei der Bestimmung der optimalen Ausgleichszahlungen und damit der Aufteilung der Effizienzgewinne im Gleichgewicht bestehen aber Unterschiede zum ursprünglichen Modell, da die Gleichgewichtsbedingungen (5.46) und (5.47) nicht mehr voneinander unabhängig sind wie die Bedingungen (5.11) und (5.12).

Die Bedingungen bilden nun ein untereinander verknüpftes System von homogenen Differenzengleichungen erster Ordnung mit zeitlich abhängigen Koeffizienten. Dieses System muß rekursiv gelöst werden, d.h. von dem letzten Angebot wird schrittweise rückwärtsgehend die Folge teilspielperfekter Angebote für alle Perioden $t=0, \ldots, T$ und damit das optimale Eröffnungsangebot $\mathrm{a}^{1}{ }_{0}{ }^{*} \mathrm{bzw} . \mathrm{a}^{2}{ }_{0}{ }^{*}$ bestimmt. Die Aufteilung der Effizienzgewinne hängt hier nicht nur davon $a b$, welche der beiden 
Regierungen die Verhandlungen beginnt, sondern auch davon, welche von beiden in der Periode $\mathrm{t}=\mathrm{T}$ ein letztes Angebot abgeben würde.

Individuell-rationales Verhalten der in dieser Situation anbietenden Regierung impliziert die Wahl des Angebots $a_{T}$ so, daß der in dieser Periode nur noch einmal realisierbare Effizienzgewinn $\Pi^{*}$ vollständig ihr zufällt. Die reagierende Regierung, für die in Periode $t=T+1$ keine Angebotschance mehr besteht und die sich damit nicht mehr durch ein eigenes Angebot verbessern kann, handelt rational, wenn sie diesem extremen Angebot zustimmt. ${ }^{151}$ Welche der beiden Regierungen den Vorteil des letzten Angebots hat, wird durch die Größe von $\mathrm{T}$ festgelegt. Ist $\mathrm{T}$ gerade, dann gibt die beginnende Regierung das erste und das letzte Angebot ab. Ist $\mathrm{T}$ ungerade übernimmt jede von beiden eine dieser beiden Rollen.

Die Aufteilung der Effizienzgewinne bei einer sofortigen Einigung ist in diesem Modell von der Größe des Zeithorizonts T abhängig. Dieser Einfluß wird in den Gleichgewichtsbedingungen (5.46) und (5.47) in den Faktoren

$$
\frac{\delta_{i}^{t}-\delta_{i}^{T+1}}{\delta_{i}^{t-1}-\delta_{i}^{T+1}}
$$

deutlich. Diese sind analog zum ursprünglichen dynamischen Modell mit ein $\mathrm{Ma} ß$ für die jeweiligen Kosten der Nichteinigung jeder Regierung. Je größer dieser Faktor ist, umso geringer sind die Kosten der Nichteinigung. Bestimmt man

$$
\begin{aligned}
\frac{\partial}{\partial T} \frac{\delta_{i}^{t}-\delta_{i}^{T+1}}{\delta_{i}^{t-1}-\delta_{i}^{T+1}} & =\frac{\delta_{i}^{T+1} r_{i} \tau\left(\delta_{i}^{t-1}-\delta_{i}^{T+1}\right)-\delta_{i}^{T+1} r_{i} \tau\left(\delta_{i}^{t}-\delta_{i}^{T+1}\right)}{\left(\delta_{i}^{t-1}-\delta_{i}^{T+1}\right)^{2}} \\
& =\frac{\delta_{i}^{T+1} r_{i} \tau\left(\delta_{i}^{t-1}-\delta_{i}^{t}\right)}{\left(\delta_{i}^{t-1}-\delta_{i}^{T+1}\right)^{2}}>0,
\end{aligned}
$$

14) Würden sich nach diesem Zeitpunkt die Ausgangsbedingungen in der Weise ändern, daß immer noch Effizienzgewinne erzielt werden könnten, aber auf anderem Niveau und bei anderen Emissionsbeschränkungen, dann muß an dieser Stelle die neue, für den danach folgenden Zeitraum gültige Aufteilung des neuen Effizienzgewinns angegeben werden. 
so zeigt sich, daß mit steigendem $\mathrm{T}$ der Faktor größer wird und somit die Kosten der Nichteinigung für beide Regierungen sinken. Bestimmen wir den Grenzwert des Faktors für $\mathrm{T}$ gegen unendlich, so gilt aufgrund $0<\delta_{\mathrm{i}}<1$,

$$
\lim _{T \rightarrow \infty} \frac{\delta_{i}^{t}-\delta_{i}^{T+1}}{\delta_{i}^{t-1}-\delta_{i}^{T+1}}=\delta_{i},
$$

Dabei ergibt sich, daß in diesem Grenzfall die Gleichgewichtsbedingungen (5.46) und (5.47) identisch zu denen in (5.11) und (5.12) sind. Somit läßt sich das ursprüngliche Modell mit unendlichem Zeithorizont als ein Spezialfall des Modells mit endlichem Zeithorizont darstellen. ${ }^{16}$

Je kleiner $\mathrm{T}$, umso größer sind die Kosten der Nichteinigung jeder Regierung. Damit macht diese Betrachtung deutlich, daß mit einer Verkürzung des Zeitraums, in dem Effizienzgewinne realisiert werden können, die Vorteilhaftigkeit der Stackelberg-Unabhängigkeitsposition steigt. Damit vergrößert sich der strategische Vorteil für denjenigen, der die Verhandlungen initiiert und ein erstes Angebot abgibt. Eine anfängliche Nichteinigung würde diese Situation noch verschärfen, da für steigendes $\mathrm{t}$ gilt

$$
\begin{aligned}
\frac{\partial}{\partial t} \frac{\delta_{i}^{t}-\delta_{i}^{T+1}}{\delta_{i}^{t-1}-\delta_{i}^{T+1}} & =\frac{-\delta_{i}^{t} r_{i} t\left(\delta_{i}^{t-1}-\delta_{i}^{T+1}\right)+\delta_{i}^{t-1} r_{i} t\left(\delta_{i}^{t}-\delta_{i}^{T+1}\right)}{\left(\delta_{i}^{t-1}-\delta_{i}^{T+1}\right)^{2}} \\
& =\frac{\delta_{i}^{T+1} r_{i} t\left(\delta_{i}^{t}-\delta_{i}^{t-1}\right)}{\left(\delta_{i}^{t-1}-\delta_{i}^{T+1}\right)^{2}}<0 .
\end{aligned}
$$

Bei anfänglicher Nichteinigung verkleinert sich mit steigendem $\mathrm{t}$ der Faktor (5.48), folglich würden, im Gegensatz zum Modell mit unendlichem Zeithorizont, die Kosten der Nichteinigung jeder Regierung von einer Verhandlungsrunde zur nächsten ansteigen.

16) Einen ähnlichen Zusammenhang konnten mit einem anderen, allgemeineren Modell Fudenberg und Levine (1983) zeigen. 


\subsubsection{Stochastische Veränderungen der Ausgangsbedingungen des Umweltsystems}

In der Realität sind Behörden bei internationalen Verhandlungen über die tatsächlichen Ausgangsbedingungen der betreffenden Umweltsysteme, über die Entwicklung von Konsum und Produktion in deren Einzugsbereichen und damit über die tatsächliche Höhe der relevanten Entsorgungskosten und Schäden bestenfalls unvollkommen informiert. Zudem werden diese Größen im Zeitverlauf vielfältigen und unvorhersehbaren Änderungen unterworfen sein.

Stellvertretend für die vielfältigen Formen allgemeiner Unsicherheit analysieren wir in diesem Abschnitt ein möglichst einfaches, im Umweltzusammenhang aber typisches Beispiel. In diesem wird ein exogenes, mit zunehmender Verhandlungsdauer ansteigendes Risiko beschrieben, wonach das Umweltsystem aus seinem stationären Gleichgewichtszustand gerät und sich die Umweltbedingungen in beiden Umweltregionen drastisch verschlechtern. Eine Einigung über Emissionsbeschränkungen auf einem gegenüber dem Status quo niedrigeren Emissionsniveau kann dieses Ungleichgewicht verhindern. ${ }^{17)}$

Dazu legen wir in diesem Modell zur Beschreibung des Verhandlungsablaufs den gleichen alternierenden Angebots- und Reaktionsproze $B$ wie in den vorausgegangenen Abschnitten zugrunde. Bei der Bewertung von Verhandlungsangeboten weichen wir aber von dem bisherigen dynamischen Modell ab.

Erstens abstrahieren wir von einer Zeitpräferenz der beiden Regierungen, um die Auswirkungen der Unsicherheit klarer herausstellen zu können. Dazu ist es notwendig, wieder auf die in (4.8) definierten vonNeumann-Morgenstern-Nutzenfunktionen $\mathrm{W}^{1}$ und $\mathrm{W}^{2}$ zurückzugreifen.

17) Die Aktualität und Realitätsnähe dieses Beispiels zeigt sich im "Umkippen" von Grenzgewässern im Saarland und auch bei langfristig irreversiblen Schäden in Gebieten, die durch "Waldsterben" bedroht sind. 
Zweitens, solange sich die beiden Regierungen während des Verhandlungsprozesses nicht auf Emissionsbeschränkungen einigen, die paretosuperior gegenüber dem Status quo sind, steigt die Wahrscheinlichkeit, daß die Assimilationskräfte des Umweltsystems nachlassen und das System seinen stationären Gleichgewichtszustand verläßt. Tritt dieser Fall ein, verursacht das einen sprunghaften Anstieg der Umweltkosten $\mathbf{K}^{1}$ und $\mathrm{K}^{2}$ durch vermehrte, nicht wiedergutzumachende Schäden. Über einen langen Zeitraum hinweg, der zur Vereinfachung des Modells als unendlich angenommen wird, zeigen umweltregulierende Maßnahmen keine Wirkung mehr: Die Anreize zum Weiterverhandeln fehlen, weil keine Effizienzgewinne mehr erzielt werden können.

Zur formalen Darstellung des Risikos nachlassender Assimilationskräfte ordnet jede der Regierungen 1 und 2 dem Eintritt der Ungleichgewichtssituation im Umweltsystem eine subjektive Wahrscheinlichkeit $\mathrm{P}^{1}\left(\Psi_{1}, \tau t\right)$ bzw. $P^{2}\left(\Psi_{2}, t t\right)$ zu, die mit zunehmender Verhandlungsdauer tt gleichfalls steigt. Wir nehmen an, daß diese Wahrscheinlichkeiten im Zeitverlauf mit einer konstanten Ereignisrate $\Psi_{1}$ für Regierung 1 und $\Psi_{2}$ für Regierung 2 wachsen. Das bedeutet, wenn es bis zu einem beliebigen Zeitpunkt tt nicht zu einer Einigung gekommen ist, dann tritt innerhalb eines marginalen Zeitraums $\varepsilon$, d.h. vom Zeitpunkt $\tau$ t bis zum Zeitpunkt $\tau t+\varepsilon$, das Ungleichgewicht mit der Wahrscheinlichkeit $\Psi_{1}$ bzw. $\Psi_{2}$ ein. Damit unterstellen wir, daß dieses Ereignis im Zeitverlauf eine exponential verteilte Zufallsgröß ${ }^{18)}$ ist und sich die Wahrscheinlichkeiten $\mathrm{P}^{1}(\tau t)$ und $\mathrm{P}^{2}(\tau t)$ somit durch die Verteilungsfunktion dieses Verteilungsmodells definieren lassen, d.h.

$$
P^{i}\left(\Psi_{i}, t t\right):=1-e^{-\Psi_{i} t t}
$$

Komplementär dazu beschreibt

$$
1-P^{i}\left(\Psi_{i}, t t\right):=e^{-\Psi_{i} t t}
$$

18) Vergleiche dazu beispielsweise Mood, Graybill und Boes (1974, 111ff.). Die Annahme einer solchen Verteilung erscheint plausibel; man verwendet dieses Modell sehr oft zur Beschreibung der Haltbarkeit und Lebensdauer von technischen Systemen. 
die Wahrscheinlichkeit für den Fall, daß sich das Umweltsystem, bis es zu einer vertraglichen Einigung zwischen den Regierungen kommt, noch in seinem stationären Gleichgewichtszustand befindet.

Wir abstrahieren in diesem Modell von einer Zeitpräferenz der beiden Regierungen zur Bewertung von Angeboten im Verlaufe des schon hinreichend bekannten alternierenden Angebots- und Reaktionsprozesses. Einigt man sich in Periode $t$ bei einem Angebot $a_{t}$, dann würde eine Einigung in Periode $t+1$ über dem Angebot $a_{t+1}=a_{t}$ den gleichen Nutzen stiften, falls bis dahin die Ungleichgewichtssituation des Umweltsystems noch nicht eingetreten ist. Die von uns in (4.3) definierten von-NeumannMorgenstern-Nutzenfunktionen $\mathrm{W}^{1}$ und $\mathrm{W}^{2}$ repräsentieren eine solche Präferenzordnung. Danach erzielen Regierung 1 und 2 in Periode $t$ bei einem Angebot $a_{t}$ einen zukünftigen Nutzen

$$
w_{i}=W^{\prime}\left(\Pi^{\prime}\left(a_{a}\right)\right)
$$$$
(i=1,2) \text {, }
$$

wenn sich das Umweltsystem noch im stationären Gleichgewichtszustand befindet.

Tritt dagegen die Ungleichgewichtssituation ein, bevor eine Einigung zustande kommt, erzielen beiden Regierungen gegenüber dem Status quo einen Verlust $\pi_{i}{ }^{\circ} \leq 0(i=1,2)$. Der in diesem Fall erreichbare Nutzen beträgt nur noch

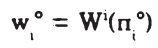$$
(i=1,2) \text {, }
$$

wobei $\mathbf{w}_{i}^{\circ} \leq \underline{w}_{i}=W^{i}(0)$ ist. $^{191}$

Unter diesen Bedingungen ist in jeder Verhandlungsrunde für die anbietende Regierung die Wahl eines bestimmten Angebots gleichzusetzen mit der Wahl einer bestimmten Lotterie. Das trifft analog auch auf die reagierende Regierung zu. Gemäß den von-Neumann-Morgenstern Annahmen,

19) Ein Fall $\pi_{1}^{\circ}=0$ kann möglicherweise in Oberlieger-Unterlieger-Situationen eintreten, in denen einzig und allein der Unterlieger von der Lngleichgewichtssituation betroffen wird, während der Oberlieger im Status quo verbleibt. 
welche die Gültigkeit der Erwartungsnutzen-Hypothese implizieren, lassen sich unter Einbezug von (5.51) und (5.52) die von uns unterstellten Präferenzordnungen der Regierungen über diese Lotterien durch Funktionen $U\left(a_{t}, t, \Psi_{i}\right)$,

$$
U^{i}\left(a_{t}, t, \Psi_{1}\right):=\left(1-P^{\prime}\left(\Psi_{i}, t t\right)\right) W^{i}\left(\Pi^{i}\left(a_{t}\right)\right)+P^{\prime}\left(\Psi_{i}, t t\right) W^{\prime}\left(n_{!}^{\circ}\right) \quad(i=1,2)
$$

repräsentieren. Sie dienen uns analog zu den Nutzenfunktionen (5.3) wieder als Zielfunktion zur Bewertung von Verhandlungsangeboten im Verlaufe des Verhandlungsprozesses.

Zur Bestimmung eines teilspielperfekten Nash-Gleichgewichts für dieses Modell betrachten wir das zu (5.4) analoge Optimierungsproblem, wenn Regierung 1 die Verhandlungen beginnt und in Periode $t$ ein Angebot $a_{t}$ abgeben soll:

$$
\begin{aligned}
& \operatorname{Max}_{a_{i} \in A^{:}} U^{1}\left(a_{t}, t, \Psi_{1}\right), \\
& U^{2}\left(a_{i}, t, \Psi_{2}\right) \geq \mathrm{C}^{2}\left(a_{t+1}, t+1, \Psi_{2}\right) \quad\left(a_{t+1} \in A^{*}, t=0,2,4, \ldots\right)
\end{aligned}
$$

Die Lösung des Optimierungsproblems läßt sich wieder durch die notwendigen Bedingungen zur Lagrangefunktion L,

$$
\begin{aligned}
L= & \left(1-P^{1}\left(\Psi_{1}, t t\right)\right) W^{1}\left(\Pi^{1}\left(a_{t}\right)\right)+P^{1}\left(\Psi_{1}, t t\right) W^{1}\left(\Pi_{1}{ }^{\circ}\right)+\lambda\left[\left(1-P^{2}\left(\Psi_{2}, t t\right)\right) W^{2}\left(\Pi^{2}\left(a_{t}\right)\right)\right. \\
& \left.+P^{2}\left(\Psi_{2}, t t\right) W^{2}\left(\Pi_{2}^{\circ}\right)-\left(1-P^{2}\left(\Psi_{2}, t(t+1)\right)\right) W^{2}\left(\Pi^{2}\left(a_{t+1}\right)\right)-P^{2}\left(\Psi_{2}, t(t+1)\right) W^{2}\left(\Pi_{2}{ }^{\circ}\right)\right]
\end{aligned}
$$

charakterisieren, wobei $\mathrm{A}$ eine nichtnegative Lagrangevariable ist. Die notwendigen Bedingungen lauten:

$$
\begin{aligned}
& \mathrm{L}_{\mathrm{e}_{1}}=\left(1-\mathrm{P}^{1}\right) \mathrm{W}^{1}{ }_{\Pi} \Pi_{\mathrm{e}_{1}}^{1}+\lambda\left(1-\mathrm{P}^{2}\right) \mathrm{W}^{2}{ }_{\pi} \Pi^{2}{ }_{\mathrm{e}_{1}}=0, \\
& \mathrm{~L}_{\mathrm{e}_{2}}=\left(1-\mathrm{P}^{1}\right) \mathrm{W}^{1}{ }_{n} \Pi^{1}{ }_{\mathrm{e}_{2}}+\lambda\left(1-\mathrm{P}^{2}\right) \mathrm{W}^{2}{ }_{\Pi} \Pi^{2}{ }_{\mathrm{e}_{2}}=0, \\
& \mathrm{~L}_{\mathrm{z}}=\left(1-\mathrm{P}^{1}\right) \mathrm{W}^{1}{ }_{\pi} \Pi^{1}{ }_{\mathrm{z}}+\lambda\left(1-\mathrm{P}^{2}\right) \mathrm{W}^{2}{ }_{\Pi} \Pi^{2}{ }_{\mathrm{z}}=0 .
\end{aligned}
$$


Als Bedingung für den komplementären Schlupf gilt

$$
\begin{aligned}
& \Lambda\left[\left(1-P^{2}\left(\Psi_{2}, t t\right)\right) W^{2}\left(\Pi^{2}\left(a_{t}\right)\right)+P^{2}\left(\Psi_{2}, t t\right) W^{2}\left(\pi_{2}{ }^{\circ}\right)\right. \\
& \left.\quad-\left(1-P^{2}\left(\Psi_{2}, \tau(t+1)\right)\right) W^{2}\left(\Pi^{2}\left(a_{t+1}\right)\right)-P^{2}\left(\Psi_{2}, t(t+1)\right) W^{2}\left(\pi_{2}{ }^{\circ}\right)\right]=0 .
\end{aligned}
$$

Unter Berücksichtigen von $\Pi^{1}{ }_{z} \equiv 1$ und $\Pi^{2}{ }_{z} \equiv-1$ vereinfacht $\operatorname{sich}(5.57) \mathrm{zu}$

$$
\left(1-P^{1}\left(\Psi_{1}, t t\right)\right) W_{n}^{1}-\lambda\left(1-P^{2}\left(\Psi_{2}, t t\right)\right) W_{n}^{2}=0 .
$$

Diese Gleichung, in (5.55) und (5.56), eingesetzt impliziert wiederum die Gültigkeit von (5.9) bzw. (4.5). Demgemäß schließt das optimale Angebot $a_{\mathfrak{t}}$ auch bei Unsicherheit gesamtkostenminimale Emissionsbeschränkungen $\left(e_{1}^{*}, e_{2}{ }^{*}\right)$ ein.

Die Nebenbedingung des Problems ist im Optimum analog zu (5.11) wieder bindend, so da $\beta$ mit Berücksichtigung der gesamtkostenminimalen Emissionsbeschränkungen $\left(e_{1}{ }^{*}, e_{2}{ }^{*}\right)$ gilt

$$
\begin{aligned}
& \left(1-P^{2}\left(\Psi_{2}, t t\right)\right) W^{2}\left(\Pi^{*}\left(e_{1}{ }^{*}, e_{2}{ }^{*}, z_{t}\right)\right)+P^{2}\left(\Psi_{2}, t t\right) W^{2}\left(\pi_{2}{ }^{\circ}\right) \\
& =\left(1-P^{2}\left(\Psi_{2}, t(t+1)\right)\right) W^{2}\left(\Pi^{2}\left(a_{t+1}\right)\right)+P^{2}\left(\Psi_{2}, t(t+1)\right) W^{2}\left(\pi_{2}{ }^{\circ}\right),
\end{aligned}
$$

womit für gegebenes $a_{t+1}$ die Ausgleichszahlungen $z_{t}$ eindeutig bestimmt sind. Ersetzt man die Wahrscheinlichkeiten gemäß (5.50),

$$
\begin{aligned}
& e^{-\Psi_{2}{ }^{\text {tt }}} w^{2}\left(\Pi^{2}\left(e_{1}^{*}, e_{2}^{*}, z_{t}\right)\right)+\left(1-e^{-\Psi_{2} \text { tt }}\right) w^{2}\left(\pi_{2}{ }^{o}\right) \\
& =\mathrm{e}^{-\Psi_{2} \tau(\mathrm{t}+1)} \mathrm{W}^{2}\left(\Pi^{2}\left(\mathrm{a}_{\mathrm{t}+1}\right)\right)+\left(1-\mathrm{e}^{-\Psi_{2} \mathrm{\tau (t+1)}}\right) \mathrm{W}^{2}\left(\Pi_{2} \mathrm{~g}\right.
\end{aligned}
$$

und vereinfacht die Bedingung durch Umformen, dann erhalten wir 


$$
W^{2}\left(\Pi^{2}\left(e_{1}^{*}, e_{2}^{*}, z_{t}\right)\right)=e^{-\Psi_{2} t} w^{2}\left(\Pi^{2}\left(a_{t+1}\right)\right)+\left(1-e^{-\Psi_{2} t}\right) W^{2}\left(\Pi_{2}{ }^{9}\right) \quad(t=0,2,4 \ldots) .
$$

Vollkommen analog dazu kann man das Optimierungsproblem von Regierung 2 in der Folgeperiode lösen. Dessen Lösung impliziert ebenfalls gesamtkostenminimale Emissionsbeschränkungen $\left(e_{1}{ }^{*}, e_{2}{ }^{*}\right)$ und eine Ausgleichszahlung $\mathrm{z}_{\mathrm{t}+1}$, die durch

$$
W^{1}\left(\Pi^{1}\left(e_{1}^{*}, e_{2}^{*}, z_{t+1}\right)\right)=e^{-\Psi_{1} t} W^{1}\left(\Pi^{1}\left(a_{t+2}\right)\right)+\left(1-e^{-\Psi_{1} t}\right) W^{1}\left(\Pi_{1}{ }^{\circ}\right) \quad(t=0,2,4 \ldots)
$$

bestimmt ist.

Die Bedingungen (5.60) und (5.61) bilden ebenso wie (5.11) und (5.12) ein System temporärer Reaktionsfunktionen, die analog zu der Argumentation in Abschnitt 5.2. untereinander als gleichwertig anzusehen sind: Das Optimierungsproblem einer Regierung, wenn diese in irgendeiner möglichen Verhandlungsrunde zur Abgabe eines Angebots aufgefordert wird, ist identisch gleich zu dem Problem, das diese in der zwei Perioden zurückliegenden Verhandlungsrunde gehabt hat. ${ }^{201}$

Die Aufteilung des Effizienzgewinns im teilspielperfekten Nash-Gleichgewicht dieses Modells wird somit mit Hilfe von

$$
\mathrm{W}^{1}\left(\Pi^{1}\left(\mathrm{a}^{2+}\right)\right)=\mathrm{e}^{-\Psi_{1} \mathrm{\tau}} \mathrm{W}^{1}\left(\Pi^{1}\left(\mathrm{a}^{1+}\right)\right)+\left(1-\mathrm{e}^{-\Psi_{1} \mathrm{t}}\right) \mathrm{W}^{1}\left(\Pi_{1}\right),
$$

$$
\mathrm{W}^{2}\left(\Pi^{2}\left(\mathrm{a}^{1+}\right)\right)=\mathrm{e}^{-\Psi_{2} \mathrm{\tau}} \mathrm{W}^{2}\left(\Pi^{2}\left(\mathrm{a}^{2+}\right)\right)+\left(1-\mathrm{e}^{-\Psi_{2} \mathrm{t}}\right) \mathrm{w}^{2}\left(\Pi_{2}\right)
$$

festgelegt. Dabei sind $\mathrm{a}^{1+}$ und $\mathrm{a}^{2+}$ die entsprechend $\mathrm{zu}$ (5.16) und (5.17) definierten Gleichgewichtsangebote, die Regierung 1 bzw. 2, wenn sie in der Stackelberg-Unabhängigkeitsposition sind, abgeben werden. Die auf

20) Der Grund dafür ist, daß die in (5.53) definierte Nutzenfunktion die Eigenschaften 1-6 unserer Zeitpräferenzordnung erfüllt und damit ebenfalls die Stationaritätseigenschaft. Vergleiche dazu auch Binmore, Rubinstein und Wolinsky (1985). 
ein solches Angebot reagierende Regierung wird diesem zustimmen, da sie sich bei einer Ablehnung in der folgenden Verhandlungsrunde nicht verbessern kann. Somit kommt es im Gleichgewicht wieder zu einer sofortigen Einigung.

In den Gleichgewichtsbedingungen (5.62) und (5.63) stellen $\mathrm{e}^{-\Psi_{1} \tau}$ und $\mathrm{e}^{-\Psi_{2} \tau}$ die Übergangswahrscheinlichkeiten für den Fall dar, daß das Umweltsystem von einer Verhandlungsrunde zur nächsten noch in seinem stationären Gleichgewichtszustand verbleibt. Es liegt dabei in der Eigenart der unterstellten Exponentialverteilung, daß diese Übergangswahrscheinlichkeiten konstant sind.

Vergleicht man nun die Bedingungen (5.62) und (5.63) mit den Gleichgewichtsbedingungen (5.14) und (5.15) des dynamischen Modells mit unendlichem Zeithorizont bei Sicherheit, dann wird deutlich, daß von den Übergangswahrscheinlichkeiten $\mathrm{e}^{-\Psi_{1} \tau}$ und $\mathrm{e}^{-\Psi_{2} \tau}$ eine sehr ähnliche Wirkung wie von den Diskontfaktoren $\delta_{1}=e^{-r_{1} t}$ und $\delta_{2}=e^{-r_{2} t}$ ausgeht.

Analog zu den marginalen Diskontraten $r_{1}$ und $r_{2}$ kann man die marginalen Ereignisraten $\Psi_{1}$ und $\Psi_{2}$ als ein individuelles Maß für den "Pessimismus" einer Regierung bezeichnen, das diese der Schnelligkeit zuordnet, mit der die Ungleichgewichtssituation des Umweltsystems eintritt. Je größer $\Psi_{1}$ und $\Psi_{2}$, umso "pessimistischer" ist eine Regierung, d.h. umso geringer sind ihre Erwartungen, vor Eintritt der Ungleichgewichtssituation im Umweltsystem eine Einigung in den Verhandlungen zu erzielen und diese gemäß dem Modell dadurch zu verhindern.

Eine "pessimistische" Einschätzung schwächt ihre Verhandlungsposition. Ihr entstehen dadurch höhere Kosten der Nichteinigung, die zu einer Verringerung ihres Effizienzgewinnanteils führen. Dieser Zusammenhang läßt sich analog zu der Diskussion in den Abschnitten 5.2. und 5.3. anhand der Gleichgewichtsbedingungen nachweisen. Da in der Ungleichgewichtssituation ein Verlust an Effizienzgewinnen auftritt und damit 


$$
\Pi_{1}^{0} \leq 0<\Pi^{\prime}\left(a^{1+}\right)
$$

gilt, verringert sich folglich mit einem Anstieg von $\Psi_{i}$ der Wert des Terms auf der rechten Seite von (5.62) bzw. (5.63). Folglich kann ceteris paribus auf der linken Seite mit steigendem $\Psi_{i}$ der entsprechende Effizienzgewinnanteil, den die andere Regierung der Regierung $\mathrm{i}$ anbietet, geringer sein.

Dieser Effekt kann darüber hinaus durch den möglichen Effizienzverlust $n_{i}^{\circ}(i=1,2)$ in der Ungleichgewichtssituation noch verstärkt werden. Je niedriger der Nutzen $W^{i}\left(n_{i}{ }^{\circ}\right)$ in diesem Fall ist, umso niedriger ist bei gegebenen Übergangswahrscheinlichkeiten wiederum der Term auf der rechten Seite der Gleichgewichtsbedingungen. Entsprechend argumentiert, je kleiner $n_{i}{ }^{\circ}$ ist, umso niedriger ist der Effizienzgewinnanteil, der Regierung i von der anderen Regierung im Gleichgewicht angeboten wird.

In diesem Modell treten analog zu der Analyse in Abschnitt 5.3. wieder die gleichen relativen Effekte zwischen der Unabhängigkeitsposition und der Abhängigkeitsposition auf. Je "pessimistischer" die beiden Regierungen sind, d.h. je größer die Raten $\Psi_{1}$ und $\Psi_{2}$ oder je größer die möglichen Effizienzverluste sind, d.h. je niedriger $n_{1}^{\circ}$ und $\pi_{2}^{\circ}$, umso größer ist der Vorteil der Unabhängigkeitspostion gegenüber der Abhängigkeitspostion. Das trifft auch bei einer Verkürzung des zeitlichen Abstands $\tau$ zwischen den Verhandlungsrunden zu.

\subsection{Zusammenfassung}

In diesem Kapitel haben wir Verhandeln als einen sequentiell ablaufenden und zeitintensiven Prozeß dargestellt, in dessen Verlauf beiden Regierungen Kosten entstehen. Diese resultieren aus dem Ablauf des Verhandlungsprozesses in einzelnen Verhandlungsrunden, die in endlichen Zeitabständen aufeinanderfolgen. Durch die Nichteinigung in einer 
Verhandlungsrunde entgeht jeder Regierung ihr Anteil an dem möglichen Effizienzgewinn in der Periode.

Im Rahmen eines dynamisch-strategischen Verhandlungsspiels wird diese Situation unter genau vorgegebenen Bedingungen für den Ablauf des Verhandlungsprozesses, d.h. mit alternierenden Verhandlungsangeboten und Reaktionen, modelliert. Den Verhandlungsparteien wird in diesem Proze $\beta$ ein individuell-rationales nutzenmaximierendes Verhalten unterstellt, wonach jeder das zukünftige Verhalten des anderen antizipiert und in seine eigenen Entscheidungen einkalkuliert.

Der einzig sinnvolle Gleichgewichtsbegriff, um ein solches Verhalten zu analysieren, ist, wie schon im Kapitel 3 skizziert. das nichtkooperative Nash-Gleichgewichtskonzept. Das im Modell angewandte teilspielperfekte Gleichgewicht stellt eine spezielle Variante dieses Konzepts dar, die der dynamischen Struktur des Modells Rechnung trägt.

Unter diesen Bedingungen zeigt sich, daß die Rahmenbedingungen der Verhandlungen für eine internationale Koordination der Umweltregulierung in unserer Modellökonomie "ideal" in der Hinsicht sind, daß diese eine effiziente Allokation begünstigen. In dem dynamischen Verhandlungsmodell unter Sicherheit, d.h. dem Modell mit unendlichem wie auch dem mit endlichem Zeithorizont, und in dem Modell mit allgemeiner Unsicherheit wird im Gleichgewicht von einer beliebigen Ausgangssituation unverzüglich (in der ersten Verhandlungsrunde) eine vertragliche Einigung auf paretooptimale Emissionsbeschränkungen erreicht. Die Modellierung des Verhandlungsproblems mit Ausgleichszahlungen sorgt zudem dafür, daß diese die zusätzliche Eigenschaft besitzen, gesamtkostenminimal zu sein.

Die Ursache für dieses effiziente Ergebnis verbirgt sich in der Annahme vollständiger Information und in den potentiellen, aber vermeidbaren Transaktionskosten. Darin sind ökonomische Anreize enthalten, sich schnell zu einigen. Somit impliziert ein individuell-rationales nutzenmaximierendes Verhalten, daß gleichzeitig die Transaktionskosten 
minimiert werden. ${ }^{21}$ Im Zusammenhang mit der Diskussion um das "Coase-Theorem" bedeuten die gewonnenen Ergebnisse: Ein kostenintensives Verhandeln individuell-rationaler nutzenmaximierender Verhandlungsparteien über die Internalisierung externer Effekte führt unter vollständiger Information zu effizienten Lösungen, die unabhängig von der initialen Verteilung der Verfügungsrechte sind, wenn sich diese potentiellen Transaktionskosten vermeiden lassen.

Bei der Aufteilung der Effizienzgewinne hat dann naturgemäß diejenige Regierung einen Vorteil, die die geringeren Opportunitätskosten der Nichteinigung aufweist und darüberhinaus die, die sich in eine strategisch bessere Position bringen kann.

Somit üben die individuellen Kosten der Nichteinigung nicht nur Anreize hinsichtlich einer schnellen Einigung aus, sondern übernehmen zugleich eine Verteilungsfunktion. Wie das Modell gezeigt hat, sind die Effizienzgewinnanteile einer Regierung umso größer, je schwächer die inländischen Zeit- und Risikopräferenzen sind, je kleiner exogene Risiken eingeschätzt werden und je weniger "pessimistisch" eine Regierung bei der Einschätzung des Ereigniszeitpunktes ist. Entstehen den Regierungen jedoch im Grenzfall keinerlei Kosten der Nichteinigung, dann ist wiederum wie im kooperativen Modell jede Aufteilung möglich. Somit sind gewisses Kosten notwendig, um eine Aufteilung erklären zu können.

Eine gewisse Unvollkommenheit des Verhandlungsmarktes besteht darin, da $\beta$ eine Verhandlungspartei zeitweise eine bessere strategische Position einnehmen kann als die andere. Im Modell ist die strategisch bessere Position die Möglichkeit, aktiv ein Angebot abzugeben, anstatt nur passiv auf ein solches zu reagieren. Dieses zeigt sich darin, daß die anbietende Regierung im Gleichgewicht einen höheren Anteil am Effizienzgewinn erhält. Der Vorteil einer solchen Position und damit das Maß der Unvollkommenheit auf dem Verhandlungsmarkt verringert sich

21) Diese Ergebnisse gelten nicht nur für den Fall vermeidbarer Verluste an zukünftigen Effizienzgewinnen, sondern auch, wie Rubinstein (1982) gezeigt hat, für den Fall mit fixen Verhandlungskosten, die jeder Verhandlungspartei pro Verhandlungsrunde entstehen. 
aber, je schneller die einzelnen Verhandlungsrunden aufeinanderfolgen. Im Grenzfall, wenn keinerlei Zeitverzögerungen entstehen, verschwindet dieser Vorteil ganz.

Das Modell mit endlichem Zeithorizont hat im Gegensatz dazu deutlich gemacht, daß mit einer Verkürzung des Zeitraums, in dem sich die Möglichkeit bietet, Effizienzgewinne zu erzielen, die Bedeutung solcher strategisch günstigen Positionen noch steigt. Das trifft auch auf das Modell mit allgemeiner Unsicherheit zu. Letzteres hat darüberhinaus gezeigt, daß je pessimistischer eine Regierung ist und je größer der Effizienzverlust ist, der ihr bei Eintritt des Ungleichgewichts im Umweltsystem entsteht, umso geringer sind die entsprechenden Effizienzgewinnanteile bei einer vertraglichen Einigung.

Die Gründe für eine schnelle Einigung sind somit bei der vollständigen Information jeder Regierung über das Verhalten der anderen zu suchen. Vollständige Information erzeugt eine endogene Sicherheit in den beiden Länderökonomien, wonach jede Regierung über die ausländischen Umweltbedingungen und Zeitpräferenzen genauso gut wie über die inländischen Gegegebenheiten informiert ist. Demgemäß hat jede Regierung eine sichere Voraussicht auf das potentielle optimale Angebots- und Reaktionsverhalten der ausländischen Regierung. Diesem kann das eigene strategische Verhalten im Verhandlungsproze $B$ entsprechend angepaßt und damit die Kosten einer Nichteinigung vermieden werden.

Unvollständige Information wäre eine realistischere und damit weniger einschränkende Annahme für die im Modell beschriebene Verhandlungssituation. Diesem zusätzlichen, in jüngster Zeit sehr intensiv in der Theorie der Verhandlungsspiele diskutierten Problem, wenden wir uns im folgenden Kapitel zu. 



\section{Die dynamisch-strategische Verhandlungslösung bei unvollstāndiger Information}

Im vorangegangenen Kapitel wurde mit Hilfe eines dynamischstrategischen Verhandlungsmodells gezeigt, daß sich bei vollständiger Information paretorelevante externe Effekte auf Verhandlungsmärkten effizient internalisieren lassen. Ein individuell-rationales nutzenmaximierendes Verhalten der Regierungen impliziert, daß die bei Hinauszögern eines Abkommens entstehenden Verhandlungskosten minimiert werden, indem man sich auf dem schnellsten Weg einigt. Welche Folgen ergeben sich aber für das Verhandlungsergebnis, wenn die Regierungen nur unvollständig informiert sind und die Beschaffung fehlender Information Zeit erfordert und zusätzliche Kosten verursacht?" 'Um dieses Problem zu untersuchen, unterstellen wir in dem dynamisch-strategischen Verhandlungsmodell eine endogene Unsicherheit in der Form asymmetrischer Information zwischen beiden Regierungen, die aber durch den Einsatz zusätzlicher Ressourcen überwunden werden kann.

Die erweiternde Annahme in diesem Kapitel besteht darin, daß die inländische Regierung zum einen über die physischen Umweltbedingungen in der ausländischen Umweltregion und zum zweiten über die Präferenzen der ausländischen Regierung schlechter als über ihre eigenen Gegebenheiten informiert ist. ${ }^{2}$ Ein individuell-rationales Verhalten impliziert für die besser informierte Regierung, den Informationsvorteil so zu nutzen, daß die eigene strategische Verhandlungsposition dabei möglichst verbessert wird. Je nach Ausgangslage ist in den Verhandlungen ein mögliches Über- oder Untertreiben der Umweltkosten sowie der Zeit- und Risikopräferenzen eine dominante Strategie, so daß für die schlechter informierte Regierung ein Problem adverser Selektion entsteht.

1) Im Zusammenhang mit Umweltproblemen ist eine verläßliche Infomationsgewinnung sehr kosten- und zeitintensiv, da insbesondere Daten über Immissionswirkungen und ihre Veränderungen nur in langfristigen, systematischen Beobachtungen gewonnen werden können. Doppelmessungen verbieten sich damit aus Effizienzgründen von selbst. Zudem haben inländische Behörden auch keine Rechte solche Messungen im Ausland selbst vorzunehmen.

2) Die Unsicherheit über die strategischen Verhaltensmöglichkeiten ist nach Harsanyi $(1967,1968)$ die dritte mögliche L'rsache von Informationsproblemen. 
In dieser Situation kann es unter bestimmten Bedingungen zu einem Informationsausgleich kommen, wenn die individuellen Anreize der besser informierten Regierung, Fehlinformation zu verbreiten, von der schlechter informierten Regierung mit Hilfe von Anreizprämien unterdrückt werden. Diese Kosten der Informationsbeschaffung stellen aber zusätzliche, potentielle Transaktionskosten dar, die Verhandlungsergebnisse beeinflussen können.

Im Rahmen des dynamisch-strategischen Verhandlungsmodells mit unendlichem Zeithorizont sollen diese Zusammenhänge näher untersucht werden. ${ }^{3)}$ Dazu beschreiben wir in Abschnitt 6.1. die zur Darstellung des Informationsproblems notwendigen Erweiterungen. Wir gehen dabei vereinfachend davon aus, daß nur eine der beiden Regierungen unvollständig über die Ausgangsbedingungen der anderen Regierung informiert ist. Bei dieser Form einseitiger Unsicherheit werden die prinzipiellen Probleme, die asymmetrische Information im Kontext von Verhandlungen aufwirft, ausreichend deutlich. Der damit verbundene formale Mehraufwand bleibt aber so gering wie möglich. Abschnitt 6.2. untersucht wieder mit Hilfe des teilspielperfekten Nash-Gleichgewichtskonzepts, welches um den Unsicherheitsaspekt erweitert werden muß, mögliche Lösungen des Modells. Diese werden in Abschnitt 6.3. zusammenfassend diskutiert.

3) Asymmetrische Information ist in der Literatur, dic Verhandlungssituationen untersucht, ein vieldiskutiertes Problem. Harris und Townsend (1981) untersuchen auf der Basis sehr allgemeiner, Riordan (1984) und Rochet (1985) mit Hilfe spezieller statischer Modelle Formen anreizkompatibler Marktmechanismen bzw. Verträge. Harsanyi und Selten (1972), Myerson und Satterthwaite (1983) sowie Myerson (1984) untersuchen das Problem mit Hilfe von axiomatischen Ansätzen. Samuelson (1980), Chatterjee und Samuelson (1981), Crawford (1982) und Riley und Zeckhauser (1983) verwenden strategische Ansätze, ohne aber dabei den typischen sequentiellen Verlauf von Verhandlungen oder eine Informationsgewinnung im Verlaufe des Verhandlungsprozesses abzubilden. Ansätze in dieser Richtung sind erst in Fudenberg und Tirole (1983), Sobel und Takahashi (1983), Cramton (1984), (1985) sowie Rubinstein (1985) zu finden. In diesen Modellen kann eine anfängliche Únsicherheit durch Weiterverhandeln vermindert werden. Sie analysieren komparativstatisch die Auswirkungen auf das Verhandlungsergebnis, wenn Verhandlungskosten, das Ausmaß der anfänglichen Unsicherheit und die Anzahl der möglichen Verhandlungsrunden variieren. In der Literatur zu grenzüberschreitenden Umweltproblemen hat einzig Smets (1974) dieses Problem aufgegriffen, geht aber von einer Situation aus, in der eine überstaatliche Autorität existiert. 


\subsection{Das dynamische Verhandlungsmodell bei unvollständiger Information}

Das Verhandlungsmodell mit unvollständiger Information baut auf dem im vorangegangenen Kapitel definierten dynamisch-strategischen Verhandlungsmodell mit unendlichem Zeithorizont auf. Die Annahmen über den Ablauf des alternierenden Angebots- und Reaktionsprozesses, die Annahmen über das Umweltsystem, sowie die Annahmen über die Zeit- und Risikopräferenzen der beiden Regierungen sind weiterhin gültig. Wir beschränken uns daher in den folgenden Unterabschnitten darauf, die notwendigen Änderungen und Erweiterungen des Modelis zu definieren und anhand von Beispielen zu erläutern. Im wesentlichen ist zu beschreiben, in welcher Form Unsicherheit für die schlechter informierte Regierung besteht, welche Anreize diese Situation bei der besser informierten Regierung auslöst und mit Hilfe welcher Methoden die schlechter informierte Regierung diese Unsicherheit beseitigen kann, d.h. auf welche Weise ein Informationstransfer im Modell möglich ist.

\subsubsection{Unvollständige Information im Verhandlungsmodell}

Grundlage der Angebotsentscheidungen der beiden Regierungen sind wiederum die in (5.3) definierten Nutzenfunktionen $U^{1}\left(a_{t}, t\right)$ und $U^{2}\left(a_{t}, t\right)$. Beide Regierungen kennen zu Beginn der Verhandlungen die tatsächlichen physischen Ausgangsbedingungen in ihren eigenen Umweltregionen sowie ihre wahren Zeit- und Risikopräferenzen. Damit sind die Regierungen wiederum über die Ausprägung ihrer eigenen Nutzenfunktion vollkommen informiert. Gegenüber dem Modell mit vollständiger Information unterstellen wir aber als zentralen Punkt dieses Modells, daß Regierung $1 \mathrm{zu}$ keinem Zeitpunkt die tatsächlichen Gegebenheiten in der Umweltregion 2 bzw. von Regierung 2 direkt beobachten kann. Für Regierung 2 bestehen dagegen keinerlei Informationseinschränkungen. 
Unter diesen Informationsbedingungen hat Regierung 2 im Vergleich zum dynamischen Verhandlungsmodell mit vollkommener Information weiterhin eine sichere Voraussicht. Für Regierung 1 besteht dagegen Unsicherheit über die wahren Verhandlungsbedingungen von Regierung 2 und damit ein Problem adverser Selektion.

Um das Informationsproblem von Regierung 1 so einfach wie möglich zu beschreiben, nehmen wir an, daß die Nutzenfunktion $U^{2}$ von Regierung 2 nur zwei verschiedene Ausprägungen aufweisen kann. Dazu unterscheiden wir zwischen $U^{21}$ und $U^{22}$ sowie entsprechend, unter Einbezug der Definitionen in (3.4), (4.1), (4.3), (5.2) sowie (5.3) zwischen $\mathrm{K}^{21}$ und $\mathrm{K}^{22}, \Pi^{21}$ und $\Pi^{22}, A^{21}$ und $A^{22}, \delta_{21}$ und $\delta_{22}$, sowie $V^{21}$ und $V^{22}$. Die verbale Darstellung wird vereinfacht, indem wir im folgenden, wenn die Ausgangsbedingungen und Präferenzen von Regierung 2 der Nutzenfunktion $U^{21}$ genügen, vom Typ 1 und wenn diese der Nutzenfunktion $U^{22}$ genügen, vom Typ 2 sprechen.

Je nachdem, welchen Typ Regierung 2 tatsächlich repräsentiert, muß Regierung 1 während des Verhandlungsprozesses möglicherweise mit unterschiedlichen Verhaltensweisen von dieser rechnen. Das erfordert analog zu der obigen Unterscheidung die entsprechenden Verhandlungsstrategien differenziert darzustellen. Demgemäß seien $G^{1}$ und $G^{2}$ die Mengen aller Strategien von Typ 1 bzw. 2. Sie enthalten entsprechend zum Grundmodell definierte Folgen von Funktionen $G^{1}=\left\{G^{1 t}\right\}_{t}=0, \ldots, \infty$ bzw. $G^{2}=\left\{G^{2 t}\right\}_{t}=0, \ldots, \infty$ für alle möglichen Perioden $t$.

Ohne die Allgemeinheit zu beschränken, unterstellen wir zur weiteren Differenzierung der beiden möglichen Ausprägungen der Nutzenfunktion von Regierung 2 die folgenden Bedingungen. Diese erlauben, die hier möglichen verschiedenen Ursachen von Unsicherheit -zum einen asymmetrische Information über die Zeit- und Risikopräferenzen von Regierung 2 und zum zweiten über die physischen Umweltbedingungen in der Umweltregion 2 - zusammen in einem Modell zu simulieren: 


\section{Annahme 1:}

(1) Für $t=0,1,2, \ldots$ sowie $a_{t}, a_{t+1} \in A^{21} \cup A^{22}$ mit der Eigenschaft $U^{21}\left(a_{t}, t\right)=U^{21}\left(a_{t+1}, t+1\right)$ sei $U^{22}\left(a_{t}, t\right) \geq U^{22}\left(a_{t+1}, t+1\right)$.

(2) Weiterhin gelte

$$
\Pi^{21}(a) \leq \Pi^{22}(a) \quad\left(a \in A^{21} \cup A^{22}\right)
$$

Gemäß dem ersten Teil der Annahme hat Regierung 2, falls sie vom Typ 2 ist, im Vergleich zu Typ 1 größere Nachteile, wenn sich eine Einigung in Verhandlungen verzögert. Typ 2 benötigt im Vergleich zwischen zwei beliebigen Verhandlungsrunden gegenüber Typ 1 ein höheres Maß an Kompensation. Dies ist gleichzusetzen mit günstigeren Emissionsbeschränkungen oder niedrigeren Ausgleichszahlungen von Land 2 an Land 1. Folglich entstehen Typ 2 vergleichsweise größere Kosten der Nichteinigung

Mit diesem Teil der Annahme ist eine höhere Zeit- und Risikopräferenz von Typ 2 im Vergleich zu Typ 1 vereinbar. Beispielsweise im Fall gleicher Risikopräferenzen und gleicher Umweltbedingungen, d.h. $\mathrm{V}^{21}=\mathrm{V}^{22}$ und $\Pi^{21}=\Pi^{22}$ läßt Annahme 1 nur noch die Möglichkeit unterschiedlicher Diskontfaktoren zu, d.h. $\delta_{21} \geq \delta_{22}$.

Bei gleichen Umweltbedingungen und gleichen Zeitpräferenzraten, d.h. $\Pi^{21}=\Pi^{22}:=\Pi^{2}$ und $\delta_{21}=\delta_{22}:=\delta_{2}$ ist Annahme $1 \mathrm{mit} \mathrm{V}^{22}=\mathrm{H}\left[\mathrm{V}^{21}\right]$ vereinbar, wobei $\mathrm{H}$ eine monoton steigende und konkave Funktion mit $\mathrm{H}(0)=0$ ist. Dies läßt sich zeigen, wenn man $\mathrm{V}^{21}=\mathrm{H}^{-1}\left[\mathrm{~V}^{22}\right]$ in (1) von Annahme 1 unter Berücksichtigung der Definition von $\mathrm{U}^{21}$ in (5.3) ersetzt:

$$
\mathrm{H}^{-1}\left[\mathrm{~V}^{22}\left(\Pi^{2}\left(\mathrm{a}_{\mathrm{t}}\right)\right)\right] \frac{\delta_{2}^{\mathrm{t}}}{1-\delta_{2}}=\mathrm{H}^{-1}\left[\mathrm{~V}^{22}\left(\Pi^{2}\left(\mathrm{a}_{\mathrm{t}+1}\right)\right)\right] \frac{\delta_{2}^{\mathrm{t}+1}}{1-\delta_{2}}
$$

bzw.

$$
v^{22}\left(\Pi^{2}\left(a_{t}\right)\right)=H\left[\delta_{2} H^{-1}\left[V^{22}\left(\Pi^{2}\left(a_{t+1}\right)\right)\right]\right]
$$


Das wiederum impliziert analog zu (5.44)

$$
\mathrm{v}^{22}\left(\Pi^{2}\left(\mathrm{a}_{\mathrm{t}}\right)\right) \geq \mathrm{V}^{22}\left(\Pi^{2}\left(\mathrm{a}_{\mathrm{t}+1}\right)\right) \delta_{2}
$$

womit die in der Annahme geforderte Eigenschaft nachgewiesen ist. Wie wir aus Abschnitt 5.3. wissen, beschreibt eine Transformation von V durch eine Funktion $\mathrm{H}$ mit diesen Eigenschaften bei gegebener Zeitpräferenzrate eine stärkeres $\mathrm{Ma} B$ an Risikoaversion. Somit ist Typ 2 in diesem Fall stärker risikoavers als Typ 1.

Welche Anreizeffekte löst eine solche Differenzierung unter den gegebenen Informationsbedingungen bei der besser informierten Regierung 2 aus? Die komparativ-statische Analyse für das Modell mit vollkommener Information hat beispielsweise gezeigt, daß die Regierung mit der geringeren Zeit- und Risikopräferenz einen Vorteil bei der Verteilung der Effizienzgewinne hat. Gelingt es dem Typ 2 von Regierung 2, in der Verhandlungssituation seine Zeit- und Risikopräferenzen gegenüber Regierung 1 glaubhaft zu untertreiben, indem er das gleiche Verhalten wie Typ 1 in den Verhandlungen zeigt, würde Typ 2 eine bessere Verhandlungsposition und damit einen größeren Anteil am Effizienzgewinn erzielen.

Der zweite Teil der Annahme stellt bestimmte Anforderungen an die Umweltkostenvorteile der beiden Typen und damit an die physischen Bedingungen innerhalb der Umweltregion 2 unter einer gegebenen rechtlichen Ausgangssituation. Danach hat Typ 1 gegenüber Typ 2 bei jedem Verhandlungsangebot $a=\left(e_{1}, e_{2}, z\right)$ einen kleineren Umweltkostenvorteil. Das ermöglicht Typ 2 von Regierung 2, wenn er sich in den Verhandlungen für Typ 1 ausgibt, einen Teil der realisierbaren Effizienzgewinne zu verschleiern und für sich zu behalten. Die Höhe dieses zusätzlichen Gewinns für Typ 2 bei einer Fehlinformation über die wahren Umweltbedingungen entspricht bei einem Angebot $a=\left(e_{1}, e_{2}, z\right)$ genau der Differenz

$$
\Pi^{22}(a)-\Pi^{21}(a)
$$


Stellt Typ 2 in Verhandlungen die Umweltkosten der eigenen Umweltregion systematisch falsch dar, kommt es gemä $\beta$ dem Verhandlungsmodell zu gesamtkostenminimalen Emissionsbeschränkungen auf der Basis der unwahren Umweltkosten. Das Verhandlungsergebnis ist in Wirklichkeit nicht paretooptimal und damit der VerhandlungsprozeB, d.h. die Folge der Verhandlungsangebote anreizunverträglich. Einigt man sich bei einem solchen Angebot, werden die externen Effekte nicht vollständig internalisiert. Die damit verbundene vertragliche Vereinbarung schreibt eine ineffiziente Umweltallokation fest.

Unter welchen Bedingungen der Anreiz besteht, die Umweltkosten falsch darzustellen und auf welche verschiedenen Arten dies sich äußern kann, wollen wir anhand eines einfachen Oberlieger-Unterlieger-Beispiels illustrieren. Es sei dazu der Fall betrachtet, in dem die Umweltregion 1 Oberlieger ist und die dort eingeleiteten Schadstoffe keinerlei Schäden innerhalb der Region verursachen. Somit reduziert sich die Umweltkostenfunktion für diese Region auf die Entsorgungskostenfunktion, d.h. es gilt $\mathrm{K}^{1}=\mathrm{C}^{1}$. Die Umweltregion 2 sei Unterlieger, ohne selbst Schadstoffe zu emittieren, so daß die Umweltkostenfunktion dort auf die Schadensfunktion reduziert ist, d.h. es gilt $\mathrm{K}^{2}=\mathrm{D}^{2}$.

Gehen wir zunächst von der dem Geschädigtenprinzip entsprechenden rechtlichen laissez-faire Situation aus, in der der Geschädigte die verursachten Schäden und die bei einer Emissionsreduzierung zusätzlichen Entsorgungskosten selbst trägt." In diesem Fall hat Regierung 2 als Unterlieger möglicherweise einen Vorteil, wenn sie die in der inländischen Umweltregion ausgelösten Schäden niedriger als in Wahrheit angeben kann. Werden die Verhandlungen dann auf der Basis der zu niedrig angegebenen Umweltschäden geführt, können auch nur die unter diesen Angaben in einem Gesamtkostenminimum erzielbaren Effizienzgewinne verteilt werden. Die Differenz zwischen wahren und angegebenen Schäden bei einer Emissionsreduktion des Oberliegers auf den Gleichgewichtswert, fällt als zusätzlicher Gewinn voll dem Unterlieger, d.h. Regierung 2, zu.

4) Siehe zur Begriffserklărung auch Fußnote 5 in Kapitel 2 


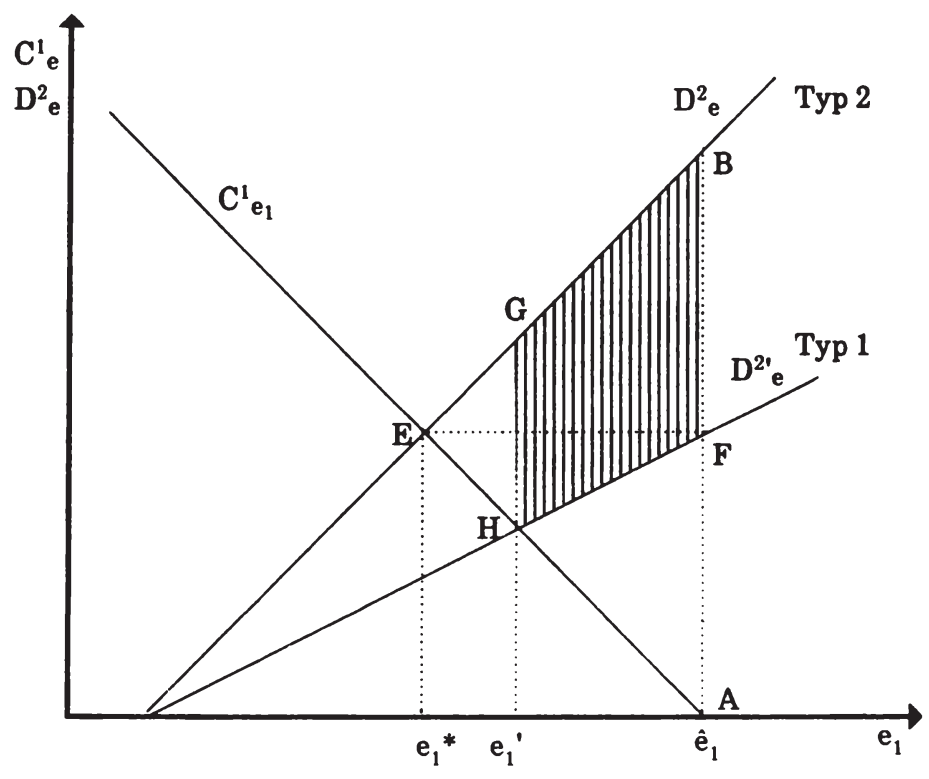

Abbildung 6.1.

Abbildung 6.1. zeigt diesen Fall graphisch. Ist Regierung 2 vom Typ 2, dann würde man sich bei vollkommener Information im Gesamtkostenminimum $e_{1}{ }^{*}$ einigen, in dem die Grenzkosten der Entsorgung $\mathrm{C}^{1}{ }_{\mathrm{e}}$ des Oberliegers gleich den Grenzschäden $\mathrm{D}^{2}{ }_{\mathrm{e}}$ des Unterliegers vom Typ 2 sind. Der durch die Emissionsreduktion vom Emissionsniveau $\hat{e}_{1}$ des Status quo auf das Emissionsniveau des Gesamtkostenminimums $e_{1}{ }^{*}$ mögliche Effizienzgewinn (die Fläche $\mathrm{ABE}$ ) wird zwischen den Ländern verteilt. Würden hingegen die Verhandlungen auf der Basis der untertriebenen Schäden $D^{2}$ geführt, die denen des Typs 1 entsprechen, einigt man sich auf $e_{1}$ '. Zur Gewinnverteilung kommt aber nur der der Fläche AFH 
entsprechende Betrag. Der realisierbare, aber nicht in die Verhandlungen einbezogene Effizienzgewinn BGHF fällt vollständig dem Unterlieger zu.

In diesem Fall kommt es zu allokativer Ineffizienz; der externe Effekt wird nicht mehr vollkommen internalisiert. Es entsteht ein Verlust an möglichen Effizienzgewinnen entsprechend der Fläche EGH. Aus der Sicht der Gesamtökonomie muß dieser als Wohlfahrtsverlust gewertet werden.

Inwieweit der Anreiz zum Untertreiben der Schäden und damit der Gefahr allokativer Ineffizienz in diesem Fall besteht, hängt aber von der Verhandlungssituation und den Zeit-und Risikopräferenzen der beiden Regierungen $a b$. Gehen wir beispielsweise von risikoneutralen, gleichen Zeitpräferenzen der beiden Regierungen mit $\delta_{1}=\delta_{21}=\delta_{22}$ und dem Grenzfall eines unendlich schnellen Verhandlungsablaufs aus, würden sich die Regierungen gemäß der dynamischen Verhandlungslösung den möglichen Effizienzgewinn genau gleich aufteilen. Ohne Untertreiben erzielt der Unterlieger dann einen Effizienzgewinnanteil entsprechend der Fläche $\mathrm{BEF}$, der sich bei Untertreiben auf einen Betrag vergrößern läßt, der der Fläche BGHF zuzüglich der Hälfte der Fläche AFH entspricht.

Dieser Anreiz verringert sich und verschwindet wieder, wenn sein Anteil am Effizienzgewinn mit sinkenden individuellen Kosten der Nichteinigung zunimmt. Entstehen Regierung 2 als Typ 2 im Grenzfall keinerlei Kosten der Nichteinigung, weil sie risikoneutral ist und keine Zeitpräferenz besitzt $\left(\delta_{22}=1\right)$, während im Gegensatz dazu Regierung 1 strikt positive Kosten entstehen, dann erhält gemäß dem Verhandlungsmodell Regierung 2 den gesamten Effizienzgewinn. Damit existiert aber auch keinerlei Anreiz zum Untertreiben mehr. Mit der wahrheitsgemäßen Schadensangabe kann Typ 2 gegenüber den Möglichkeiten bei Untertreiben den Wohlfahrtsverlust, d.h. die Fläche EGH, als zusätzlichen privaten Gewinn für sich realisieren.

Bei dem zum Geschädigtenprinzip alternativen Zivilhaftungsprinzip, demgemäß der Oberlieger für die von ihm verursachten Schäden voll aufkommen muß - solange dieses Recht nicht vertraglich eingeschränkt 


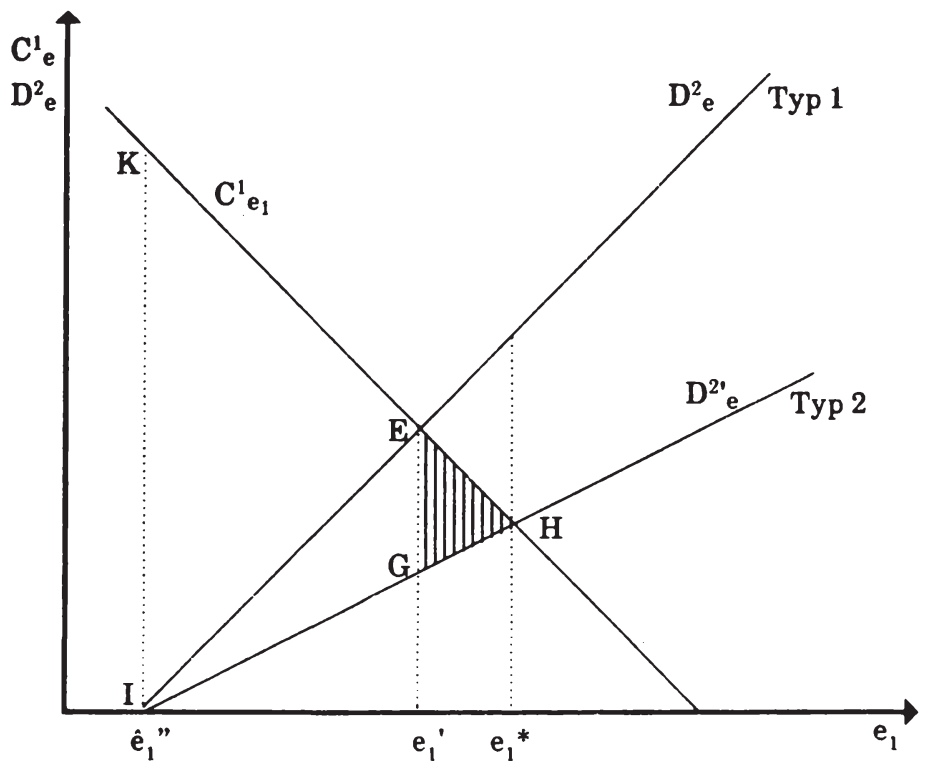

Abbildung 6.2.

wird - kehrt sich der mögliche Anreiz zum Untertreiben in einen Anreiz zum Übertreiben um. Abbildung 6.2. skizziert diese Situation, in der im Nichteinigungsfall der Unterlieger nicht geschädigt werden darf; die adäquaten Referenzkosten sind somit $\mathrm{K}^{2}\left(\hat{\mathrm{e}}_{1}{ }^{\prime}\right)=0$. Übertreibt der Unterlieger die Schäden, einigt man sich in Verhandlungen auf das Emissionsniveau $e_{1}{ }^{\prime}$. Zur Gewinnverteilung kommt nur der der Fläche IEK entsprechende Betrag. Den Effizienzgewinnanteil EGI realisiert der Unterlieger für sich alleine. ${ }^{5 \prime}$

5) Diese Phănomene lassen sich bei reinen innerstaatlichen Lmweltproblemen auch am Beispiel unterschiedlicher staatlicher Politiken beobachten. Bei einer UmweltsteuerLösung besteht ein Anreiz zur Lntertreibung der individuellen Entsorgungskosten, bei einer Standard-Lösung ein Anreiz zu übertreiben. Vergleiche dazu Weitzman (1974) und Roberts und Spence (1976). 
Der gleiche Anreiz zum Übertreiben besteht, wenn Verhandlungen auf der Basis des Verursacherprinzips - der Verursacher trägt die wohlfahrtsoptimalen Entsorgungskosten und der Geschädigte die restlichen Schäden - stattfinden. Im Oberlieger-Unterlieger-Fall entspricht diese Verhandlungssituation "moral suasion" ohne Ausgleichszahlungen. Durch Übertreiben würde ein niedrigeres als das tatsächlich gesamtkostenminimale Emissionsniveau erreicht, wodurch der Unterlieger seine Schäden gegenüber dem optimalen Niveau zusätzlich verringern könnte.

Bei einem Tausch der Oberlieger- und der Unterliegerrolle zwischen den beiden Regierungen läßt sich auf ebenso einfache Weise mit den gleichen Argumenten zeigen, daß in den Fällen mit Ausgleichszahlungen für den Oberlieger die genau entgegengesetzten Anreize zu den oben geschilderten auftreten. Beispielsweise besteht in der laissez-faire Situation dann ein Anreiz zum Übertreiben der Entsorgungskosten. In den Fällen ohne Ausgleichszahlungen besteht dagegen weiterhin ein allgemeiner Anreiz zum Übertreiben dieser Kosten.

Damit wird deutlich, daß asymmetrische Information in Verhandlungssituationen ein strategisches Problem für die besser wie auch die schlechter informierte Regierung darstellt. Die besser informierte Regierung muß darüber entscheiden, ob die Wahrheit oder ein Über- bzw. Untertreiben für sie vorteilhafter ist. Die schlechter informierte Regierung hat das Problem herauszufinden, welchen Anreiz die besser informierte Regierung in der jeweiligen Situation nun eigentlich hat. D.h. für sie besteht möglicherweise der Anreiz, die fehlende Information zu erhalten. Generell gilt aber, daß jeglicher Anreiz zur Falschdarstellung aus der Sicht der Gesamtökonomie die Gefahr einer ineffizienten Umweltallokation im Verhandlungsergebnis in sich birgt. 


\subsubsection{Informationstransfer im Verhandlungsmodell}

Die Kommunikation zwischen den Regierungen vor und während des Verhandlungsprozesses ist auf Angebote und die entsprechenden Reaktionen beschränkt, so daß die Möglichkeiten für einen die Unsicherheit vermindernden Informationstransfer nur innerhalb dieses Rahmens liegen. Regierung 1 ist somit gezwungen, zu Beginn der Verhandlungen willkürlich oder auf der Basis exogener Information Vermutungen über den wahren Typ von Regierung 2 anzustellen, die sie während des Verhandlungsprozesses laufend aktualisiert. ${ }^{61}$

Vermutungen werden mit Hilfe subjektiver Wahrscheinlichkeiten beschrieben. Es sei die Wahrscheinlichkeit q $(0 \leq q \leq 1)$ das Maß, mit dem Regierung $1 \mathrm{zu}$ Verhandlungsbeginn vermutet, daß Regierung 2 vom Typ 1 ist. Entsprechend beschreibt $1-q$ die Vermutung, gegen Typ $2 \mathrm{zu}$ verhandeln. Bevor Regierung 2 eine erste Angebots- oder Reaktionsentscheidung getroffen hat, bilden diese Wahrscheinlichkeiten die Grundlage der Angebotsentscheidung von Regierung 1.

Während des Verhandlungsprozesses können sich aufgrund von dessen gegebener Struktur die Vermutungen nur nach Angeboten bzw. nach Reaktionen von Regierung 2 ändern. Solange Regierung 1 selbst am Zuge ist, erhält sie keine neuen Informationen über Regierung 2. Folglich bleiben während dieser Zeit ihre Vermutungen unverändert. Aufgrund des vollständigen Erinnerungsvermögens der Regierungen sind die aktualisierten Vermutungen in jeder Verhandlungsperiode $t$, beschrieben durch die Wahrscheinlichkeiten $\left(q_{t}, 1-q_{t}\right)$, von allen bisherigen abgelehnten Angeboten bis zu dem letzten Angebot von Regierung 2 abhängig."

6) Daß Vermutungen nach Infomationsgewinnen laufend aktualisiert werden müssen, ist eine Forderung, die aus Savage's (1954) Axiomen für Entscheidungen unter Unsicherheit abgeleitet ist.

7) Bedingt durch den wechselseitigen Angebotsprozeß können sich natürlich Vermutungen auch schon direkt nach einer bestimmten Angebotsreaktion von Regierung 2 veråndern. Sie sind aber zu diesem Zeitpunkt noch nicht entscheidend. Anders dagegen im Modell von Sobel und Takahashi (1983), das ebenfalls auf einseitiger Unsicherheit aufbaut, aber nur eine einseitige Angebotsabgabe zuläßt. Dort findet eine Aktualisierung nur nach den Angebotsreaktionen des Nachfragers, d.h. zwischen den Verhandlungsrunden statt. 
Die Werte dieser Wahrscheinlichkeiten $\left(q_{t}, 1-q_{t}\right)$ werden somit durch Funktionen $Q^{t}$ für alle möglichen Verhandlungsperioden $t$ bestimmt, die bei Beginn der Verhandlungen durch Regierung 1 für gerade $t$ durch

$$
Q^{t}:\left(a_{0}, \ldots, a_{t-1}\right) \rightarrow[0,1]
$$

und für ungerade $t$ durch

$$
Q^{t}:\left(a_{0}, \ldots, a_{t}\right) \rightarrow[0,1]
$$

definiert sind. Für gerade $t$ gilt dabei $Q^{\mathfrak{t}}=Q^{t-1}$, und es gilt zudem $q=q_{0}=Q^{0}$. Beginnt dagegen Regierung 2, werden die Funktionen $Q^{t}$ für gerade $t$ durch

$$
Q^{t}:\left(a_{0}, \ldots, a_{t}\right) \rightarrow[0,1]
$$

und für ungerade $t$ durch

$$
\mathbf{Q}^{\mathrm{t}}:\left(\mathrm{a}_{\mathbf{0}}, \ldots, \mathrm{a}_{\mathrm{t}-1}\right) \rightarrow[0,1]
$$

definiert, wobei für ungerade $t Q^{t}=Q^{t-1}$ gilt.

Das vollständige System von Vermutungen wird parallel zu den Folgen der Angebots- und Reaktionsentscheidungen von Regierung 1 durch entsprechende Folgen dieser subjektiven Wahrscheinlichkeiten beschrieben. Dazu enthalte die Menge $\Omega$ alle möglichen Folgen von Wahrscheinlichkeiten, die Regierung 1 dem Typ 1 und dazu komplementär dem Typ 2 von Regierung $2 \mathrm{zu}$ Beginn und während aller möglichen Verhandlungsprozesse zuordnen kann. Ein Element aus $\Omega$ sind zwei Folgen $\Omega_{1}$ und $\Omega_{2}$, die jeweils aus einer initialen Wahrscheinlichkeit $q$ bzw. 1 -q und Funktionen $Q^{t}$ bzw. $1-Q^{t}$ für alle möglichen Perioden $t=0,1,2, \ldots$ bestehen:

$$
\begin{aligned}
& \Omega_{1}=\left\{\mathbf{q}, \mathbf{Q}^{0}, \mathbf{Q}^{1}, \ldots\right\} \\
& \Omega_{2}=\left\{1-\mathbf{q}, 1-\mathbf{Q}^{0}, 1-\mathbf{Q}^{1}, \ldots\right\} .
\end{aligned}
$$


Die Folgen $\Omega_{1}$ und $\Omega_{2}$ dieser Wahrscheinlichkeiten und damit die Funktionen $Q^{\mathfrak{t}}$ müssen näher spezifiziert werden, d.h. es muß bestimmt werden, unter welchen Bedingungen ein Informationstransfer von Regierung 2 zu Regierung 1 zustande kommt. Unter den gegebenen Kommunikationsmöglichkeiten bieten sich dazu zwei Ansatzpunkte. Beide bauen auf der Überlegung auf, daß eine über die initialen Vermutungen hinausgehende Diskriminierung der zwei Typen unmöglich erscheint, solange sich die beiden unter der gegebenen Anreizstruktur in ihrem typspezifischen Angebots- und Reaktionsverhalten nicht direkt unterscheiden. Verhält sich Regierung 2, falls sie vom Typ 2 ist, konsistent wie der möglicherweise für sie vorteilhaftere Typ 1, dann kann Regierung 1, die den wahren Typ nicht kennt, niemals mit Bestimmtheit feststellen, gegen welchen Typ sie tatsächlich verhandelt.

Der erste Ansatzpunkt besteht darin, daß das Angebots- und Reaktionsverhalten der Regierungen unter bestimmten Bedingungen die Funktion eines Signals oder eines Selbstselektionsmechanismus bekommen kann. ${ }^{81}$ Ein Angebot von Regierung 2 hat die Funktion eines Signals, mit dem diese ihren Typ glaubhaft versichert, wenn ihr Angebot nur für einen der beiden Typen individuell-rational ist, für den anderen dagegen nicht. Regierung 1, die dieses Verhalten beobachtet, kann daraus einen Rückschluß auf den wahren Typ ziehen. Ein Angebot von Regierung $1 \mathrm{mit}$ Selbstselektionsfunktion baut auf dem gleichen Prinzip auf. Es trennt Typ 1 von Typ 2, wenn es bei einem solchen Angebot nur für einen Typ individuell-rational ist, diesem Angebot zuzustimmen.

8) Selbstselektionsmechanismen und Signale sind zwei Varianten des von Hurwicz (1972) beschriebenen Konzepts anreizkompatiblen Verhaltens, demgemäß sich Wirtschaftssubjekte mit unterschiedlichen Eigenschaften voneinarder trennen lassen. Dieses Konzept hat weite Verbreitung in der Literatur gefunden. Vergleiche beispielsweise zu Informationsproblemen auf Arbeitsmärkten Spence (1974), Hart (1983), auf Versicherungsmärkten Rothschild und Stiglitz (1976), auf Finanzmärkten Jaffee und Russell (1976), Stiglitz und Weiss (1981). Sehr ausgeprägt ist dieses Konzept auch in der Social-Choice Literatur zu finden. Vergleiche dazu den C̈berblicksartikel von Dasgupta, Hammond und Maskin (1979) und den Sarnmelband von Green und Laffont (1979). Myerson (1984), Riordan (1984) und Rochet (1985) benutzen es, um anreizkompatible Verträge zu beschreiben. Rubinstein (1985) beschreibt damit erstınals Anreizkompatibilität von Verhandlungsprozessen in einem dynamisch-strategischen Modell, das ein einfaches Teilungsproblem behandelt. 
In diesem Sinne treffen wir eine Annahme, die den Zusammenhang zwischen Beobachtungen von Regierung 1 und ihren Vermutungen näher beschreibt, d.h. die Wahrscheinlichkeiten $q_{t}$ näher spezifiziert. Dabei gehen wir davon aus, daß die.Vermutungen über den wahren Typ nach einer Einigung und somit Beendung des Verhandlungsprozesses unbedeutend sind und folglich nicht näher beschrieben werden müssen:91

\section{Annahme 2:}

Falls für $q_{0}=q$ oder für ein $q_{t}$ mit $0<q_{t}<1, t \in(1,2,3, \ldots)$ die Angebote $a_{t}$ und $a_{t+1}$ die folgenden Eigenschaften besitzen:

$$
\begin{aligned}
& U^{21}\left(a_{t}, t\right)<U^{21}\left(a_{t-1}, t+1\right), \\
& U^{22}\left(a_{t}, t\right) \geq U^{22}\left(a_{:-1}, t+1\right),
\end{aligned}
$$

so gelte

$$
q_{t+1}=q_{t+2}=\ldots=1 .
$$

Bei den Eigenschaften

$$
U^{2 h}(a, t) \leq U^{2 h}\left(a_{t-1}, t+1\right)
$$$$
(h=1,2)
$$

gelte

$$
q_{t+1}=q_{t}
$$

Diese Annahme baut auf den im vorangegangenen Abschnitt definierten Unterschieden zwischen Typ 1 und Typ 2 auf, wonach Regierung 2, wenn sie vom Typ 2 ist, möglicherweise einen Vorteil daraus ziehen kann, wenn sie ihr spezifisches Angebots- und Reaktionsverhalten dem von Typ 1 anpaßt.

Ein von Regierung 1 in Periode $t$ abgegebenes Angebot $a_{t}$ hat in dieser Situation Selbstselektionseigenschaften, wenn die vom Typ 1 erwartete Ablehnung des Angebots und das von ihm in der Periode $t+1$ erwartete Angebot $a_{t+1}$ den imitierenden Typ 2 schlechterstellt. Das gilt für alle Angebote, die in Annahme 2 den Eigenschaften (6.1) genügen. Auf der Basis des von Typ 1 erwarteten Verhaltens "trennt" somit die Reaktion

9) Das trifft zu, wenn Regierung 1 in Periode $t$ ein Angebot a abgibt, dem bei individuell-rationalen Verhalten beide Typen zustimmen, $\mathbf{d} . \mathrm{h}$. mit den Eigenschaften

$$
U^{2 h}\left(a_{i}, t\right) \geq \mathrm{C}^{32 h}\left(a_{i+1}, t+1\right) \quad(h=1,2) .
$$


von Regierung 2 die beiden Typen. Für Typ 2 ist es vorteilhaft, das Angebot $a_{t}$ anzunehmen. Für Typ 1 dagegen ist es vorteilhaft, abzulehnen und weiter zu verhandeln. Es sei an dieser Stelle angemerkt, daß der umgekehrte Fall, Wahl des Angebots $a_{t}$ so, daß Typ 1 annimmt und Typ 2 ablehnt und weiter verhandelt, im Widerspruch zu Annahme 1 steht.

Regierung 1 sieht ihre Erwartungen aber erst dann sicher bestätigt, wenn Regierung 2 das von Typ 1 erwartete Angebot $a_{t+1}$ auch tatsächlich abgibt. Sie setzt damit ein Signal, mit dem sie ihren Typ glaubhaft versichert. Erst danach vermutet Regierung 1 mit Sicherheit gegen Typ 1 zu verhandeln. Wir unterstellen, daß nur solche Vermutungen, die mit Sicherheit auf einen bestimmten Typ hinweisen, im weiteren Verlauf des Verhandlungsprozesses stationär sein können.

Genügt dagegen das Angebot $a_{t}$ nur den Ungleichungen (6.2) würde Regierung 2 unabhängig von ihrem Typ erwartungsgemäß ablehnen. Das Verhalten der beiden Typen unterscheidet sich nicht, folglich können sich die Vermutungen auch nicht ändern.

Diese in Annahme 2 formulierte Informationsregel erfüllt das Bayessche Gesetz. Ist Regierung 2 vom Typ 1 und lehnt ein Angebot ab, welches (6.1) genügt und gibt in Periode $t+1$ ein Angebot $a_{t+1} a b$, dann ist die Wahrscheinlichkeit dieses Ereignisses gleich eins und damit gleich der neuen a posteriori Wahrscheinlichkeit.

Ein zweiter Ansatzpunkt für einen Informationstransfer bietet sich, wenn Regierung 1 im Verlaufe des Verhandlungsprozesses bei Regierung 2 ein inkonsistentes Verhalten beobachtet, das sie in diesem Moment nicht erwartet. Damit wird eine Bedingung an das Verhalten von Regierung 2 im Gleichgewicht geknüpft. Es gelte folgende weitere Annahme:

\section{Annahme 3:}

Es sei $0<q, q_{t}<1$ für ein $t \in(0,1,2, \ldots)$. Regierung 1 erwartet von Typ 1 der Regierung 2 ein Angebot $a_{t}$ in Periode t. Gibt Regierung 2 ein Angebot $a_{t}^{\prime} \neq a_{t} a b$, dann gilt $q_{t}=q_{t+1}=\ldots=0$. 
Diese Annahme geht ebenso davon aus, da $B$ unter der gegebenen Anreizstruktur Regierung 1 von Regierung 2, unabhängig von deren tatsächlichem Typ, ein Verhalten erwartet, welches spezifisch für Typ 1 ist. Beobachtet sie nun dagegen ein Verhalten, das von diesem typspezifischen Verhalten abweicht, dann vermutet sie mit Sicherheit in den folgenden Verhandlungsrunden gegen Typ 2 zu verhandeln.

Die Informationsregel in Annahme 3 enthält eine Drohung an Regierung 2, bei einem beobachteten inkonsistenten Verhalten im weiteren Verhandlungsverlauf von der für sie schlechtesten Situation auszugehen. Zum einen "diszipliniert" Regierung 1 damit Typ 1 zu einem typspezifischen Verhalten, womit auch ein durch Unachtsamkeit verursachter Verlust an möglichen Effizienzgewinnen verhindert werden soll. Zum anderen zwingt diese Annahme Typ 2 in Verhandlungssituationen, in denen Über- oder Untertreiben für ihn suboptimal sein sollte, dies entweder konsistent weiter zu tun oder von Anfang an die Wahrheit zu sagen.

Die Informationsregeln in den Annahmen 2 und 3 stehen hier stellvertretend für alle die Moglichkeiten, aus denen sich bei asymmetrischer Information Rückschlüsse auf fehlende Informationen ziehen lassen. In unserem Fall läßt sich damit das Risiko von Regierung 1 , gegen einen anderen als den von ihr vermuteten Typ zu verhandeln, nicht ganz ausschalten. Diese Regeln erfüllen aber den Zweck, dieses Risiko zu minimieren.

\subsection{Das teilspielperfekte Bayessche Nash-Gleichgewicht}

Die Mengen $F, G^{1}$ und $G^{2}$ der möglichen Verhandlungsstrategien, die Nutzenfunktionen $\mathrm{U}^{1}, \mathrm{U}^{21}$ und $\mathrm{U}^{22}$ sowie die Menge $\Omega$ der möglichen Vermutungen von Regierung 1 bilden analog zu Kapitel 5 die Ausgangsbedingungen eines nichtkooperativen Mehrperioden-Verhandlungsspiels in extensiver Form. Zur Bestimmung von Lösungen dieses Spiels verwenden wir wiederum das Konzept des teilspielperfekten Nash-Gleichgewichts. 
Der Gleichgewichtsbegriff kann aber in der bisher definierten Form nicht direkt auf das jetzige Modell übertragen werden. Die durch die Informationsasymmetrie begründete einseitige Unsicherheit von Regierung 1 stellt darin ein neues Element dar, ganz im Gegensatz zu dem im Abschnitt 5.4. unterstellten exogenen Risiko. Dort bestand gleichermaßen für beide Regierungen ein Anreiz, das mit der Zeit wachsende Risiko zu vermeiden. In diesem Modell dagegen, verhalten sich insbesondere dann, wenn die Ausgangsbedingungen von Regierung 2 denen des Typs 2 entsprechen, die Interessen beider Regierungen entgegengesetzt zueinander. Dieses führt zu einer wesentlichen qualitativen Veränderung des individuell-strategischen Verhaltens beider Regierungen. Vermutungen von Regierung 1 und ihre gezielten Veränderungen mit Hilfe bestimmter Angebots- und Reaktionsentscheidungen stellen dabei sich ergänzende Komponenten dar.

Wir gehen davon aus, daß die durch Annahme 2 und 3 spezifizierten Vermutungen $\Omega_{1}$ und $\Omega_{2}$ sowie die Strategien $F, G^{1}$ und $G^{2}$ konsistent sind. Damit läßt sich nun die Gleichgewichtsdefinition wie folgt den neuen Ausgangsbedingungen anpassen:

\section{Definition:}

Die Kombination $\left(F^{*}, G^{t *}, G^{2 *}, \Omega_{1}^{*}, \Omega_{2}^{*}\right)$ aus reinen Strategien und Vermutungen des in Periode $t=0$ beginnenden Mehrperioden-Verhandlungsspiels in extensiver Form gilt als teilspielperfekter Bayesscher NashGleichgewichtspunkt, wenn in jedem seiner in $t=1,2, \ldots$ beginnenden möglichen Teilspiele, die aus $\left(F^{*}, G^{1 *}, G^{2 *}, \Omega_{1}{ }^{*}, \Omega_{2}{ }^{*}\right)$ abgeleiteten Strategien und Vermutungen, Gleichgewichtspunkt dieses Teilspiels sind. ${ }^{101}$

10) Das auf diese Art definierte teilspielperfekte Gleichgewicht stellt einen Speziaifall eines "sequentiellen Gleichgewichts" nach der Definition von Kreps und Wilson (1982) dar. Indem sie Verhaltensstrategien unterstellen, verwenden Kreps und Wilson einen allgemeineren Strategiebegriff, benutzen aber eine schärfere Konsistenzbedingung zwischen Vermutungen und Strategien als die von uns unterstellte Konsistenz im Sinne der Bayesschen Regel. Da sich unser Modell auf reine Strategien beschränkt, wird diese Einschränkung wieder hinfällig. 
In einem teilspielperfekten Nash-Gleichgewicht dieses verallgemeinerten Typs muß folglich für Regierung 1 gelten: Ihre Vermutungen und ihr Angebots- bzw. Reaktionsverhalten maximieren unter Berücksichtigung des darunter im weiteren Verlauf der Verhandlungen erwarteten individuell-rationalen Verhaltens von Regierung 2 in jeder möglichen Verhandlungsrunde ihren erwarteten Gesamtnutzen.

Für die unter Sicherheit agierende Regierung 2 gilt entsprechend dem Modell mit vollkommener Information: Ihr Angebots- bzw. Reaktionsverhalten maximiert typspezifisch in jeder Verhandlungsrunde unter Berücksichtigung des im weiteren Verhandlungsverlauf individuellrationalen Verhaltens von Regierung 1 ihren Gesamtnutzen.

Kreps und Wilson (1982) haben gezeigt, daß für ein Modell dieser Art ein teilspielperfektes Bayessches Nash-Gleichgewicht $\left(F^{*}, G^{1 *}, G^{2 *}, \Omega_{1}{ }^{*}, \Omega_{2}{ }^{*}\right)$ existiert. Mögliche teilspielperfekte Verhandlungsgleichgewichte können demnach, analog zu unserem Vorgehen in Kapitel 5, durch die Lösungen der um die Informationsbedingungen erweiterten Entscheidungsprobleme der beiden Regierungen beschrieben werden. Diese Probleme lassen sich dabei so analysieren, als ob sie den entsprechenden Problemen zur Bestimmung eines teilspielperfekten Gleichgewichts bei vollkommener Information überlagert sind.

Die möglichen Lösungen dieser um den Informationsaspekt erweiterten Entscheidungsprobleme werden dabei zum einen mitbestimmt, ob Typ 2 von Regierung 2 unter den gegebenen Ausgangsparametern einen Anreiz zur Fehlinformation hat und sich für Typ 1 ausgibt. Ist dies der Fall, bestimmen diese Parameter ebenso, ob Regierung 1 einen Vorteil gewinnen kann, wenn sie über den wahren Typ von Regierung 2 mehr erfährt, als es ihre anfänglichen Vermutungen (q, $1-q)$ zulassen.

Hat Typ 2 von Regierung 2 keinen Anreiz zum Über- oder Untertreiben, würden die Verhandlungen, da diese Tatsache Regierung 1 ebenso bekannt ist, wie in der Situation bei vollkommener Information ablaufen. Diesen Fall brauchen wir somit nicht weiter zu untersuchen. Den dazu 
alternativen Fall, in dem ein solcher Anreiz besteht, wollen wir genauer betrachten. Er ist im Zusammenhang mit der Diskussion über die Einflüsse von Transaktionskosten auf Verhandlungsergebnisse der entscheidendere.

Hat Regierung 1 den Anreiz mehr über Regierung $2 \mathrm{zu}$ erfahren, bietet sich ihr mit Annahme 2 nur eine Möglichkeit: Ein Trennangebot setzt dem imitierenden Typ 2 einen Anreiz sich vom Typ 1 durch eine schnelle Zustimmung $z u$ unterscheiden, während es für Typ 1 rational ist, abzulehnen und weiter zu verhandeln. Eine Alternative dazu bieten nichttrennende Angebote, denen beide Typen sofort zustimmen würden. Damit könnte Regierung 1 das Unsicherheitsproblem umgehen und eine schnelle Einigung erreichen. In diesen beiden Alternativen ist ein Tradeoff-Problem für beide Regierungen verborgen. Die damit verbundenen zentralen Fragen lauten: Ist es für Regierung 1 vorteilhaft, wenn ein Informationstransfer stattfindet und die beiden Typen von Regierung 2 voneinander getrennt werden? Wenn ja, unter welchen Ausgangsbedingungen ist es für Regierung 2 vorteilhaft und auch möglich, diese Trennung zu vermeiden?

Falls Regierung 2 vom Typ 1 ist und ein solches Angebot ablehnt, entstehen beiden Regierungen im Vergleich zu einem nichttrennenden Angebot Kosten der Nichteinigung. Regierung 1 entstehen zudem weitere Kosten, weil sie durch den Wechsel der Anbieterrolle die günstigere Unabhängigkeitsposition aufgeben muß. Ist dagegen Regierung 2 vom Typ 2, erreicht Regierung 1 mit Hilfe dieses Trennangebots einen höheren Anteil am Effizienzgewinn als mit einem nichttrennenden Angebot.

Dieser Trade-off $z$ wischen einem Trennangebot und einem nichttrennenden Angebot stellt sich in diesem Modell für beide Regierungen als ein diskretes Entscheidungsproblem dar. Dieses besteht aufgrund der Kosten der Nichteinigung sofort in der ersten Verhandlungsrunde am Anfang der Periode $t=0$. 
Wir untersuchen zunächst das Entscheidungsproblem von Regierung 1. Dazu sei mit $\mathrm{a}^{1 \mathrm{n}}$ das optimale nichttrennende Angebot bezeichnet, mit $\mathbf{a}^{\text {1s }}$ das optimale Trennangebot und mit $\mathrm{a}^{21}$ das optimale Angebot, mit dem Typ 1 (und aufgrund des Imitierungsvorteils ebenso Typ 2) von Regierung 2 auf solche Angebote antworten würde. Der erwartete Nutzen von Regierung 1 bei einem Trennangebot $\mathrm{a}^{1 \mathrm{~s}}$ ist

$$
\mathrm{qU}^{1}\left(\mathrm{a}^{21}, \mathrm{t}+1\right)+(1-q) \mathrm{C}^{-1}\left(\mathrm{a}^{15}, \mathrm{t}\right) \quad(\mathrm{t}=0,1)
$$

und bei einem nichttrennenden Angebot $\mathrm{a}^{1 \mathrm{n}}$

$$
q U^{1}\left(a^{1 n}, t\right)+(1-q) U^{1}\left(a^{2 n}, t\right)=U^{11}\left(a^{1 n}, t\right)
$$$$
(t=0,1) .
$$

Wir betrachten zunächst den einfacheren Fall, in dem das Angebot $a^{1 \text { n }}$ vorteilhafter ist. D.h. mit (6.3) und (6.4) unter Berücksichtigung der Definition von $U^{1}$ gilt

$$
V^{1}\left(\Pi^{1}\left(a^{1 n}\right)\right)>q V^{1}\left(\Pi^{i}\left(a^{21}\right)\right) \delta_{1}+(1-q) V^{1}\left(\Pi^{1}\left(a^{15}\right)\right)
$$

Die Lösung dieses Falles, d.h. die Bestimmung von $\mathrm{a}^{1 \mathrm{n}}$ und $\mathrm{a}^{21}$, ist identisch $z u$ der eines Verhandlungsmodells bei vollkommener Information, worin Regierung 1 gegen Typ 1 von Regierung 2 verhandelt.

Beginnt Regierung 1 die Verhandlungen und gibt aus der Unabhängigkeitsposition heraus am Anfang der ersten Periode $t=0$ ein nichttrennendes Angebot $\mathrm{a}^{1 \mathrm{n}}$ ab, dann löst dieses das Maximierungsproblem:

$$
\begin{aligned}
& \operatorname{Max}_{a_{0} \in A^{1}} \quad U^{1}\left(a_{0}, 0\right) \\
& U^{21}\left(a_{0}, 0\right) \geq U^{21}\left(a_{1}, 1\right), \\
& U^{22}\left(a_{0}, 0\right) \geq U^{22}\left(a_{1}, 1\right)
\end{aligned}
$$$$
\left(a_{1} \in A^{21}\right) \text {. }
$$

Dabei erwartet Regierung 1 von Regierung 2 in Periode $t=1$ ein Angebot $a_{1}=a^{21}$, welches das Problem löst: 


$$
\begin{aligned}
& \operatorname{Max}_{a_{1} \in A^{21}} \quad U^{21}\left(a_{1}, 1\right) \\
& \mathrm{U}^{1}\left(\mathrm{a}_{1}, 1\right) \geq \mathrm{U}^{1}\left(\mathrm{a}_{2}, 2\right) \quad\left(\mathrm{a}_{2} \in \mathrm{A}^{1}\right) .
\end{aligned}
$$

Aufgrund der in Annahme 1 vereinbarten unterschiedlichen Eigenschaften der Nutzenfunktionen $U^{21}$ und $U^{22}$ muß bei gegebenem $a_{1}$ in einem Maximum des Problems (6.6) die erste der beiden Nebenbedingungen bindend sein. Der umgekehrte Fall oder der Fall, daß beide Nebenbedingungen bindend sind, steht im Widerspruch zur Annahıne 1. Damit ist gezeigt, daß die Lösung dieses Problems und auch des in (6.7) vollkommen analog zu den Maximierungsproblemen (5.4) und (5.5) auszuwerten sind. Wie in Abschnitt 5.3. hinlänglich erörtert wurde, werden beide Probleme von einem nichttrennenden Angebot $a_{0}=a^{1 n}$,

$$
a^{\ln }:=\left(e_{1}{ }^{i *}, e_{2}{ }^{1 *}, z^{1 n}\right),
$$

und von einem entsprechenden Gegenangebot $a_{1}=a^{21}$,

$$
\mathbf{a}^{21}:=\left(\mathbf{e}_{1}^{i *}, \mathbf{e}^{i *}, \mathbf{z}^{21}\right),
$$

gelöst. Die Emissionsbeschränkungen $e_{1}{ }^{1 *}$ und $e_{2}{ }^{1 *}$ besitzen dabei die Eigenschaft, den aggregierten Effizienzgewinn, der sich mit Typ 1 realisieren läßt, zu maximieren, d.h.

$$
\left(\mathbf{e}_{1}^{1 *}, \mathbf{e}_{2}{ }^{1 *}\right)=\underset{\mathbf{e}_{1}, \mathrm{e}_{2}}{\operatorname{Arg} \operatorname{Max}}\left(\Pi^{\mathrm{i}}+\Pi^{2:}\right),
$$

Das ist identisch zu einer Minimierung der aggregierten Umweltkosten.

Die Ausgleichszahlungen $z^{1 \mathrm{n}}$ und $\mathrm{z}^{21}$ werden entsprechend $\mathrm{zu}(5.14)$ und (5.15) implizit durch die Gleichgewichtsbedingungen

$$
\begin{aligned}
& V^{1}\left(\Pi^{1}\left(e_{1}{ }^{1 *}, e_{2}{ }^{1 *}, z^{21}\right)\right)=V^{1}\left(\Pi^{1}\left(e_{1}^{1 *}, e_{2}{ }^{1 *}, z^{1 n}\right)\right) \delta_{1}, \\
& V^{21}\left(\Pi^{21}\left(e_{1}{ }^{1 *}, e_{2}{ }^{1 *}, z^{10}\right)\right)=V^{21}\left(\Pi^{21}\left(e_{1}{ }^{1 *}, e_{2}{ }^{1 *}, z^{22}\right)\right) \delta_{21}
\end{aligned}
$$

bestimmt. 
Beginnt dagegen Regierung 2 die Verhandlungen und erwartet von Regierung 1 ein nichttrennendes Angebot $\mathrm{a}^{\text {1n }}$, dann wird sie analog zur Betrachtung in Abschnitt 5.2. im Gleichgewicht die Verhandlungen am Anfang der Periode $t=0$ mit einem Angebot $a_{0}=a^{21}$ eröffnen.

Wenn sich die Regierungen bei einem gemäß (6.8) oder (6.9) bestimmten Gleichgewichtsangebot $\mathrm{a}^{\text {ln }}$ bzw. $\mathrm{a}^{21}$ einigen, verhält sich Regierung 1 gegenüber Typ 1 wie in Verhandlungen bei vollkommener Information und ignoriert das Anreizproblem. Unterscheiden sich in dieser Situation die beiden Typen von Regierung 2 in den Ausgangsbedingungen für das Umweltsystem, d.h. es gilt $\Pi^{22}>\Pi^{21}$, dann führt dieses anreizunverträgliche Verhalten, falls Regierung 2 vom Typ 2 ist, zu Effizienzverlusten in der Gesamtökonomie. Die Emissionsheschränkungen $e_{1}{ }^{1 *}$ und $e_{2}{ }^{1 *}$ als Bestandteile der Angebote $\mathrm{a}^{1 \mathrm{n}}$ und $\mathrm{a}^{21}$ sind dann nicht mehr paretooptimal. Diese werden von Typ 2 wie auch von Regierung 1 um ihres persönlichen Vorteils willen gegenüber Emissionsbeschränkungen $e_{1}{ }^{2 *}$ und $\mathrm{e}_{2}{ }^{2 *}$ mit der Eigenschaft

$$
\left(e_{i}^{* *}, e^{* * *}\right)=\underset{e_{i}, e_{2}}{\operatorname{Arg} M a x}\left(\Pi^{1}+\Pi^{* 2}\right),
$$

vorgeschlagen bzw. akzeptiert. Die Emissionsbeschränkungen $e_{1}{ }^{2 *}$ maximieren somit den aggregierten Effizienzgewinn, der sich mit Typ 2 realisieren läßt. Damit ist gezeigt, daß ein individuell-rationales Verhalten in Verhandlungssituationen bei asymmetrischer Information zu ineffizienten Verhandlungsergebnissen führen kann.

Der Fall, in dem das Trennangebot $\mathrm{a}^{1 \mathrm{~s}}$ im Vergleich zu einem Angebot $\mathrm{a}^{1 \mathrm{n}}$ für Regierung 1 vorteilhafter ist und entgegen (6.5) gilt

$$
\mathrm{V}^{1}\left(\Pi^{1}\left(a^{1 \mathrm{n}}\right)\right)<q \mathrm{~V}^{1}\left(\Pi^{1}\left(\mathrm{a}^{21}\right)\right) \delta_{1}+(1-q) \mathrm{V}^{1}\left(\Pi^{1}\left(\mathrm{a}^{15}\right)\right)
$$

ist komplexer in der Darstellung. Es besteht im Gleichgewicht ein wesentlicher Unterschied darin, ob Regierung 1 ein solches Angebot $a^{1 s}$ 
vor oder nach einem ersten Angebot von Regierung 2 abgibt und damit, ob Regierung 1 oder Regierung 2 die Verhandlungen beginnt.

Betrachten wir zunächst wieder den Fall, daß Regierung 1 zuerst die Unabhängigkeitsposition einnimmt und am Anfang von Periode $t=0$ ein erstes Angebot $a_{0}$ abgibt, welches Trenneigenschaften haben soll. Die Wahl dieses Angebots ist im Gegensatz zur Wahl des Angebots $a^{1 n}$, aufgrund der Unsicherheit über die Reaktion von Regierung 2, gleichbedeutend mit der Wahl einer bestimmten Lotterie. Eines der möglichen Ergebnisse dieser Lotterie wird durch das strategische Verhalten von Typ 1 bestimmt, das andere Ergebnis gilt es unter Berücksichtigung der "Trennbedingung" (6.1) zu maximieren.

Danach löst das optimale Trennangebot $\mathrm{a}^{1 \mathrm{~s}}$ unter den gemäß Annahme 2 in (6.1) genannten Voraussetzungen das Maximierungsproblem:

$$
\begin{aligned}
& \operatorname{Max}_{a_{11} \in A} \quad q U^{-1}\left(a_{1}, 1\right)+(1-q) U^{1}\left(a_{10}, 0\right) \\
& U^{-2 i}\left(a_{0}, 0\right)<U^{21}\left(a_{1}, 1\right) \\
& U^{-22}\left(a_{0}, 0\right) \geq U^{22}\left(a_{1}, 1\right)
\end{aligned}
$$$$
\left(a_{1} \in A^{2 \mathrm{i}}\right)
$$

Regierung 1, die nach einer Ablehnung ihres Angebots mit Sicherheit Typ 1 vermutet, erwartet von diesem in Periode $t=1$ zur Bestätigung ein Angebot, welches das Maximierungsproblem löst:

$$
\begin{aligned}
& \operatorname{Max}_{a_{1} \in A^{21}} \quad U^{21}\left(a_{1}, 1\right), \\
& U^{1}\left(a_{1}, 1\right) \geq U^{1}\left(a^{11}, 2\right)
\end{aligned}
$$

Dabei ist das Angebot $\mathrm{a}^{11}$ dasjenige, welches Regierung 1 in einem teilspielperfekten Gleichgewicht abgeben würde, wenn sie in Regierung 2 mit Sicherheit Typ 1 vermutet. Das Maximierungsproblem (6.16) ist somit ein Spezialfall von (6.7). Ein Angebot $a_{1}$ welches (6.16) maximiert, wird folglich durch (6.10) und (6.11) bestimmt und ist identisch zu dem Angebot $a^{21}$ in (6.9), d.h. es gilt $a_{1}=a^{21}$.

Unter diesen Bedingungen reduziert sich das Problem (6.15) und wird unter Berücksichtigen der Definitionen von $U^{1}, U^{21}$ sowie $U^{22}$ durch die 
notwendigen Bedingungen $\mathrm{zu}$ der folgenden Lagrangefunktion $\mathrm{L}$ bestimmt:

$$
\begin{aligned}
L= & q V^{1}\left(\Pi^{1}\left(a^{21}\right)\right) \frac{\delta_{1}}{1-\delta_{1}}+(1-q) V^{1}\left(\Pi^{1}\left(a_{0}\right)\right) \frac{1}{1-\delta_{1}} \\
& +\lambda_{1}\left[V^{21}\left(\Pi^{21}\left(a^{21}\right)\right) \frac{\delta_{21}}{1-\delta_{21}}-V^{21}\left(\Pi^{21}\left(a_{0}\right)\right) \frac{1}{1-\delta_{21}}\right] \\
& +\lambda_{2}\left[V^{22}\left(\Pi^{22}\left(a_{0}\right)\right) \frac{1}{1-\delta_{22}}-V^{22}\left(\Pi^{22}\left(a^{21}\right)\right) \frac{\delta_{22}}{1-\delta_{22}}\right] .
\end{aligned}
$$

Dabei sind $\lambda_{1}$ und $\lambda_{2}$ nichtnegative Lagrangevariable. Die notwendigen Bedingungen lauten:

$$
L_{e_{1}}=(1-q) V_{n}^{1} \Pi_{e_{1}}^{1} \frac{1}{1-\delta_{1}}-\lambda_{1} v_{n}^{21} \Pi_{e_{1}}^{21} \frac{1}{1-\delta_{21}}+\lambda_{2} v_{n}^{22} \Pi_{e_{1}}^{22} \frac{1}{1-\delta_{22}}=0,
$$

$$
L_{e_{2}}=(1-q) V_{\pi}^{1} \Pi_{e_{2}}^{1} \frac{1}{1-\delta_{1}}-\Lambda_{1} V_{\pi}^{21} \Pi_{e_{2}}^{21} \frac{1}{1-\delta_{21}}+\Lambda_{2} V_{n}^{22} \Pi_{e_{2}}^{22} \frac{1}{1-\delta_{22}}=0,
$$

$$
L_{z}=(1-q) v_{n}^{1} \Pi_{z}^{1} \frac{1}{1-\delta_{1}}-\Lambda_{1} v_{n}^{21} \Pi_{z}^{21} \frac{1}{1-\delta_{21}}+\lambda_{2} v_{n}^{22} \Pi_{2}^{22} \frac{1}{1-\delta_{22}}=0
$$

Als Bedingung für den komplementären Schlupf gilt

$$
\lambda_{1}\left|\mathrm{v}^{21}\left(\Pi^{21}\left(\mathrm{a}^{21}\right)\right) \frac{\delta_{21}}{1-\delta_{21}}-\mathrm{v}^{21}\left(\Pi^{21}\left(\mathrm{a}_{0}\right)\right) \frac{1}{1-\delta_{21}}\right|=0,
$$

$$
\Lambda_{2}\left|\mathrm{~V}^{22}\left(\Pi^{22}\left(\mathrm{a}_{0}\right)\right) \frac{1}{1-\delta_{22}}-\mathrm{V}^{22}\left(\Pi^{22}\left(\mathrm{a}^{21}\right)\right) \frac{\delta_{22}}{1-\delta_{22}}\right|=0
$$


Aufgrund $\Pi^{1} \equiv 1$ und $\Pi^{21}{ }_{z} \equiv \Pi_{z}^{22} \equiv-1$ vereinfacht sich (6.19) zu

$$
L_{z}=(1-q) V_{n}^{1} \frac{1}{1-\delta_{1}}+\lambda_{1} V_{n}^{21} \frac{1}{1-\delta_{21}}-\lambda_{2} V_{n}^{22} \frac{1}{1-\delta_{22}}=0
$$

Aus dieser Bedingung wird deutlich, daß eine Lösung nur mit $\lambda_{1}=0$ und $\Lambda_{2}>0$ vereinbar ist, da gemäß Annahme 1 nur eine Nebenbedingung bindend sein kann. Berücksichtigt man dieses und setzt (6.21) in (6.17) sowie (6.18) ein, führt das zu

$$
\begin{aligned}
& \mathrm{II}^{1}{ }_{e_{1}}+\Pi^{22}{ }_{e_{1}}=0, \\
& \Pi_{e_{2}}^{1}+I^{22}{ }_{e_{2}}=0 .
\end{aligned}
$$

Damit sind die in (6.13) definierten Emissionsbeschränkungen $e_{1}{ }^{2 *}$ und $\mathrm{e}_{2}{ }^{2 *}$ diejenigen, die das Maximierungsproblem (6.15) lösen, d.h. Emissionsbeschränkungen, die den aggregierten Effizienzgewinn auf der Basis der Umweltkosten von Typ 2 maximieren.

Die Höhe der Ausgleichszahlungen $z^{1 s}$ des optimalen Angebots a ${ }^{1 s}$ werden aufgrund $\Lambda_{2}>0$ durch die im Optimum bindende zweite Nebenbedingung bestimmt, d.h. mit (6.20) gilt

$$
\mathrm{V}^{22}\left(\Pi^{22}\left(\mathrm{e}_{1}^{2 *}, \mathrm{e}_{2}^{2 *}, \mathrm{z}^{15}\right)\right)=\mathrm{V}^{23}\left(\Pi^{22}\left(\mathrm{a}^{21}\right)\right) \delta_{22} \text {. }
$$

Damit ist das optimale Trennangebot $\mathbf{a}^{1 \mathrm{~s}}$,

$$
a^{1 s}:=\left(e_{1}{ }^{2 *}, e_{2}^{2 *}, z^{1 s}\right)
$$

vollständig charakterisiert.

Gibt in einem Gleichgewicht Regierung 1 ein solches Angebot $\mathrm{a}^{1 \mathrm{~s}} \mathrm{ab}$, welches von Typ 2 erwartungsgemä $B$ angenommen, dagegen von Typ 1 abgelehnt wird, dann kommt es auf jeden Fall zu einer paretooptimalen Umweltregulierung, bei der die internationalen externen Effekte voll- 
kommen internalisiert werden. Die Einigung dazu erfolgt, falls Regierung 2 vom Typ 1 ist, spätestens in der zweiten Verhandlungsrunde.

Bei der Verteilung der Effizienzgewinne muß Regierung 1 gemäß (6.21) Typ 2 mindestens den Nutzen zugestehen, den dieser mit seinem den Typ 1 imitierenden Verhalten im weiteren Verlauf der Verhandlungen hätte erreichen können. Die Ausgleichszahlungen von Land 2 an Land 1 verringern sich somit im Fall asymmetrischer Information gegenüber dem bei vollkommener Information um eine Anreizprämie, die notwendig ist, um den Anreiz zum Über- bzw. Untertreiben zu unterdrücken.

Wir betrachten nun den Fall, daß Regierung 2 zuerst die Unabhängigkeitsposition einnimmt und am Anfang von Periode $t=0$ ein erstes Angebot $a_{0}$ abgibt. In dieser Situation stellt sich die Frage, ob ein Angebot $\mathrm{a}_{0}=\mathrm{a}^{21}$ für Regierung 2 in jedem Fall das optimale erste Angebot ist?

Ein solches Eröffnungsangebot impliziert in Verbindung mit (6.11) und (6.14)

$$
\mathrm{V}^{1}\left(\Pi^{1}\left(\mathrm{a}^{21}\right)\right)=\mathrm{V}^{1}\left(\Pi^{1}\left(\mathrm{a}^{1 \mathrm{r}}\right)\right) \delta_{1}<\mathrm{qV}^{1}\left(\Pi^{1}\left(\mathrm{a}^{21}\right)\right) \delta_{1}{ }^{2}+(1-q) \mathrm{V}^{\mathrm{i}}\left(\Pi^{\mathrm{i}}\left(\mathrm{a}^{15}\right)\right) \delta_{1} .
$$

Folglich würde es von Regierung 1 abgelehnt und mit einem Trennangebot $\mathrm{a}^{1 \mathrm{~s}}$ in Periode $\mathrm{t}=1$ beantwortet. Dabei entstehen Kosten der Nichteinigung, die für Regierung 2 möglicherweise größer als der Verlust sind, den sie in Kauf nehmen muß, wenn sie ein Angebot $a_{0}$ wählt, welches das Maximierungsproblem

$$
\begin{aligned}
& \operatorname{Max}_{a_{n} \in A^{21}} \quad U^{21}\left(a_{0}, 0\right) \\
& U^{1}\left(a_{n}, 0\right) \geq q U^{1}\left(a^{21}, 2\right)+(1-q) U^{1}\left(a^{15}, 1\right)
\end{aligned}
$$

löst. Dieses sei mit $a^{2 n}$ bezeichnet. Es besitzt die Eigenschaft, daß dabei Regierung 1 gerade den erwarteten zukünftigen Nutzen erreicht. Damit setzt Regierung 2 Regierung 1 einen Anreiz, sofort zu einer Einigung zu kommen, und verhindert dadurch den Informationsgewinnungsproze $B$. Löst man das Maximierungsproblem (6.24) analog zu dem Problem (6.7), 
dann impliziert ein solches Angebot $a^{2 n}$ gemä $B$ (6.10) Emissionsbeschränkungen $e_{1}{ }^{1 *}$ und $e_{2}{ }^{1 *}$, die den Effizienzgewinn auf der Basis von Typ 1 maximieren. Die optimalen Ausgleichszahlungen $\mathrm{z}^{2 \mathrm{n}}$ sind in diesem Fall durch die Bedingung

$$
V^{1}\left(\Pi^{1}\left(e_{1}^{1 *}, e_{2}^{1 *}, z^{2 a}\right)\right)=q V^{1}\left(\Pi^{1}\left(a^{21}\right)\right) \delta_{1}{ }^{2}+(1-q) V^{1}\left(\Pi^{1}\left(a^{1 s}\right)\right) \delta_{i} \cdot
$$

festgelegt. Gemäß (6.10) und (6.25) wird das optimale Angebot $\mathrm{a}^{2 \mathrm{n}}$ definiert durch

$$
\mathbf{a}^{2 n}:=\left(e_{1}{ }^{1 *}, e_{2}{ }^{1 *}, z^{2 n}\right)
$$

Das Angebot $a^{21}$ wie auch das Angebot $a^{2 n}$ können bei Beginn der Verhandlungen durch Regierung 2, je nach den Ausgangsbedingungen im Umweltsystem sowie den Zeit- und Risikopräferenzen der beiden Regierungen, das teilspielperfekte erste Angebot von Regierung 2 sein. Ähnlich wie die diskreten Entscheidungsmöglichkeiten bei Regierung 1, ob diese die beiden Typen von Regierung 2 trennen soll, stellt sich bei Beginn der Verhandlungen durch Regierung 2 und einem Trennvorteil für Regierung 1 für Regierung 2 das diskrete Entscheidungsproblem, ob sie sich trennen lassen soll oder nicht. Die Entscheidung darüber hängt wiederum von der dabei erreichbaren Nutzenposition ab.

Regierung 2 wird ein Angebot $\mathrm{a}^{21}$ abgeben, wenn Typ 1 einen "Signalisierungsvorteil" hat, d.h. wenn es für ihn vorteilhaft ist, daß Regierung 1 ihn in Regierung 2 auch mit Sicherheit vermutet. In einem solchen Fall muß

$$
\mathrm{V}^{21}\left(\Pi^{21}\left(\mathrm{a}^{2 \mathrm{n}}\right)\right)<\mathrm{V}^{21}\left(\Pi^{21}\left(\mathrm{a}^{21}\right)\right) \delta_{21}{ }^{2}
$$

gelten. Dies ist bemerkenswert, weil sich für Regierung 2 der Vorteil, zu Beginn der Verhandlungen die Stackelberg-Unabhängigkeitsposition einzunehmen, mit einem solchen Signalisierungsvorteil für Typ 1 in einen Nachteil umkehrt. Stünde Regierung 2 entgegen den Regeln des Verhandlungsspiels die Möglichkeit offen, das erste Angebot an Regierung 1 
abzutreten, dann würden sich beide Typen im Gleichgewicht bei den gleichen Angeboten, d.h. $\mathrm{a}^{21}$ im Falle von Typ 1 und $\mathrm{a}^{1 \mathrm{~s}}$ im Falle von Typ 2, aber eine Verhandlungsrunde früher einigen. Entsprechend ihren Zeitpräferenzen würden sich dabei beide Regierungen besserstellen.

Ist dagegen für Typ 1 eine schnelle Einigung, ohne von Regierung 1 diskriminiert zu werden, vorteilhafter, weil entgegen (6.27) gilt

$$
V^{21}\left(\Pi^{21}\left(a^{21}\right)\right)>V^{21}\left(\Pi^{21}\left(a^{21}\right)\right) \delta_{21}{ }^{2},
$$

dann besteht wiederum die Gefahr eines ineffizienten nicht paretooptimalen Verhandlungsergebnisses. Das Angebot $\mathrm{a}^{2 \mathrm{n}}$ schließt Emissionsbeschränkungen $\mathrm{e}_{1}{ }^{1 *}$ und $\mathrm{e}_{2}{ }^{1 *}$ ein, die, falls Regierung 2 vom Typ 2 ist, suboptimal sind. Auch hier ist das eigennützige Verhalten der beiden Regierungen das ausschlaggebende Motiv dieser Ineffizienzen.

In der Frage, ob Regierung 1 Vorteile hat, wenn die beiden Typen von Regierung 2 voneinander getrennt werden und ebenso bei dem entsprechenden Problem von Regierung 2, ob sie dies verhindern möchte, spielen die initialen Vermutungen von Regierung 1 eine zentrale Rolle. Wenn wir von der Voraussetzung (6.14) für ein Trennangebot $\mathrm{a}^{\text {1s }}$ ausgehen und beziehen darin die Gleichgewichtsbedingung (6.11) unter Berücksichtigen von (6.8) und (6.9) ein, so gilt

$$
V^{1}\left(\Pi^{1}\left(a^{1 n}\right)\right)<q V^{1}\left(\Pi^{1}\left(a^{\ln }\right)\right) \delta_{1}^{2}+(1-q) V^{1}\left(I I^{1}\left(a^{1 s}\right)\right) .
$$

Auflösen nach q ergibt

$$
q<\frac{v^{1}\left(\Pi^{1}\left(a^{1 s}\right)\right)-V^{1}\left(\Pi^{1}\left(a^{\ln }\right)\right)}{V^{1}\left(\Pi^{1}\left(a^{1 s}\right)\right)-V^{1}\left(\Pi^{1}\left(a^{1 n}\right)\right) \delta_{1}^{2}} .
$$

Der Ausdruck auf der rechten Seite der Ungleichung beschreibt eine obere Schranke für die Vermutungen in Form der Wahrscheinlichkeit q, bis zu der ein Trennangebot $a^{1 s}$ für Regierung 1 vorteilhaft erscheint. Je weniger sie in Regierung 2 Typ 1 vermutet, d.h. je kleiner q, umso eher wird sie 
bereit sein, die Typen von Regierung 2 durch ein Angebot $a^{1 s}$ voneinander zu trennen. Dieser Anreiz steigt natürlich ebenso, je größer der dabei mögliche Zuwachs an Effizienzgewinnanteilen

$$
\Pi^{1}\left(a^{15}\right)-\Pi^{1}\left(a^{2 n}\right)
$$

ist. Sind die Vermutungen von Regierung 1 rational in der Hinsicht, da $\beta$ sie die tatsächlichen Bedingungen widerspiegeln, dann besteht mit steigenden Erwartungen, gegen Typ 2 zu verhandeln, auch ein Anreiz, Regierung 2 durch ein Trennangebot $\mathrm{a}^{\mathrm{i}_{\mathrm{s}}} \mathrm{zu}$ diskriminieren.

Eine ähnliche Aussage läßt sich für Typ 1 von Regierung 2 in der Frage treffen, ob ein "Signalisierungsvorteil" besteht, d.h. eine Trennung gemä $\beta$ (6.27) vorteilhaft erscheint. Je kleiner die initiale Wahrscheinlichkeit q, mit der Typ 1 von Regierung 1 vermutet wird, umso größer ist der erwartete Nutzen von Regierung 1. Damit steigt auch der Nutzen, den Regierung 2, will sie eine Diskriminierung verhindern, Regierung $1 \mathrm{im}$ Gleichgewicht gemäß (6.25) zugestehen muß. Das wird erreicht durch eine höhere Ausgleichszahlung von Land 2 an Land 1. Folglich verringert sich der Nutzen $V^{21}\left(\Pi^{21}\left(a^{2 n}\right)\right)$ von Regierung 2 in dieser Situation, und es kommt gemäß (6.27) eher zu einem Angebot $\mathbf{a}^{21}$, welches eine Trennung von Regierung 2 durch Regierung 1 auslöst.

Die möglichen Ergebnisse von Verhandlungen bei unvollständiger Information unter unterschiedlichen Ausgangsbedingungen wollen wir wiederum anhand des schon im Modell mit vollkommener Information verwendeten Beispiels illustrieren. Dabei ergibt sich die Gelegenheit, die Entscheidungsprobleme der beiden Regierungen, d.h. ob ein Trennen der beiden Typen vorteilhaft ist oder nicht, und damit die Frage, ob allokative Ineffizienzen auftreten können, besser im Zusammenhang betrachten zu können. Den Regierungen wird dazu Risikoneutralität unterstellt, so daß sich ihre Präferenzen durch die Nutzenfunktionen (5.19) repräsentieren lassen. 
Unter diesen Bedingungen vereinfacht sich die Ungleichung (6.28) zu

$$
q<\frac{\Pi^{1}\left(a^{18}\right)-\Pi^{1}\left(a^{1 n}\right)}{\Pi^{1}\left(a^{1 s}\right)-\Pi^{1}\left(a^{1 n}\right) \delta_{1}^{2}}
$$

Für Typ 1 von Regierung 2 ist eine Diskriminierung vorteilhaft, wenn mit (6.27) gilt

$$
\Pi^{21}\left(\mathrm{a}^{2 n}\right)<\Pi^{21}\left(\mathrm{a}^{21}\right) \delta_{2:}{ }^{2} .
$$

Berücksichtigt man, daß

$$
\Pi^{1}\left(\mathbf{a}^{2 n}\right)+\Pi^{21}\left(a^{2 n}\right) \equiv I^{1}\left(a^{21}\right)+\Pi^{21}\left(a^{21}\right),
$$

folgt nach Einsetzen in die Ungleichung

$$
\Pi^{1}\left(\mathrm{a}^{2 n}\right)>\left(1-\delta_{21}^{2}\right) \Pi^{21}\left(\mathrm{a}^{21}\right)+\Pi \Pi^{2}\left(\mathrm{a}^{21}\right) .
$$

Mit (6.25) gilt im Gleichgewicht

$$
\Pi^{\prime}\left(a^{: n}\right)=q \Pi^{1}\left(a^{21}\right) \delta_{1}{ }^{2}+(1-q) \Pi^{1}\left(a^{: i}\right) \delta_{i:}
$$

Setzt man die Gleichgewichtsbedingung in (6.30) ein und löst nach q auf, so folgt

$$
\mathrm{q}<\frac{\Pi^{1}\left(\mathrm{a}^{1 \mathrm{~s}}\right) \delta_{1}-\Pi^{\mathrm{l}}\left(\mathrm{a}^{21}\right)-\left(1-\delta_{21}^{2}\right) \Pi^{21}\left(\mathrm{a}^{21}\right)}{\Pi^{1}\left(\mathrm{a}^{1 \mathrm{~s}}\right) \delta_{1}-\Pi^{1}\left(\mathrm{a}^{21}\right) \delta_{1}^{2}} .
$$

Mit (6.11) ergibt sich daraus

(6.31)

$$
q<\frac{\Pi^{1}\left(a^{i s}\right)-\Pi^{1}\left(a^{\ln }\right)-\frac{\left(1-\delta_{21}^{2}\right)}{\delta_{1}} \Pi^{21}\left(a^{21}\right)}{\Pi^{1}\left(a^{15}\right)-\Pi^{1}\left(a^{1 n} \delta_{1}^{2}\right.} .
$$


Im direkten Vergleich zwischen (6.29) und (6.31) zeigt sich, daß die obere Schranke für die Vermutungen von Regierung 1 , bis zu der sich eine Trennung lohnt, strikt größer ist, als die entsprechende Schranke für Typ 1 von Regierung 2. Es lassen sich somit drei Bereiche für die initialen Vermutungen von Regierung 1 abgrenzen.

Für die initiale Wahrscheinlichkeit q, die beide Ungleichungen erfüllt, erscheint für beide Regierungen eine Trennung vorteilhaft. Beginnt Regierung 1, wird sie daher ein Trennangebot $\mathrm{a}^{1 \mathrm{~s}}$ abgeben; beginnt Regierung 2, wird diese ein Angebot $\mathrm{a}^{21}$ abgeben, welches zunächst von Regierung 1 abgelehnt und in der folgenden Verhandlungsrunde mit einem Trennangebot $\mathrm{a}^{1 \mathrm{~s}}$ beantwortet wird.

Bei einer Wahrscheinlichkeit q, die nur die Ungleichung (6.29) erfüllt, wird Regierung 1, wenn sie beginnt, wiederum ein Angebot $\mathrm{a}^{1 \mathrm{~s}}$ abgeben. Dagegen wird Regierung 2, wenn sie beginnt, eine Trennung vermeiden wollen und ein Angebot $\mathrm{a}^{2 \mathrm{n}}$ abgeben.

Ist q größer als die Werte beider Schranken, gibt Regierung 1 als erstes das Angebot $\mathrm{a}^{\mathrm{ln}} \mathrm{ab}$, womit sie eine Trennung umgeht. Beginnt Regierung 2, würde sie diese Situation ausnutzen und das für ihre Nutzenposition ingesamt vorteilhafteste Angebot $\mathrm{a}^{21}$ abgeben.

Die Aufteilungen der Effizienzgewinne bei den jeweilig möglichen Angeboten sind durch die entsprechenden Gleichgewichtsbedingungen bestimmt und lassen sich explizit angeben. Hat Regierung 1 , wenn $q$ beide Ungleichungen (6.29) und (6.31) nicht erfüllt, den Anreiz zu einer sicheren schnellen Einigung, dann ergibt sich entsprechend zu Abschnitt 5.2. aus (6.11) und (6.12), falls Regierung 2 vom Typ 1 ist, die Aufteilung

$$
\left(\Pi^{1}\left(a^{1 n}\right), \Pi^{21}\left(a^{\ln }\right)\right)=\left(\frac{1-\delta_{21}}{1-\delta_{1} \delta_{21}} \pi^{1 *}, \frac{\left(1-\delta_{1} \delta_{21}\right.}{1-\delta_{1} \delta_{21}} \pi^{1 *}\right),
$$


wobei $\pi^{1 *}$ der maximale Effizienzgewinn in der Ökonomie bei einem tatsächlichen Typ 1 ist, d.h.

$$
\Pi^{1 *}=\Pi^{1}\left(e_{1}^{1 *}, e_{2}^{1 *}, z\right)+\Pi^{21}\left(e_{1}^{1 *}, e_{2}^{1 *}, z\right)
$$

Ist in dieser Situation Regierung 2 vom Typ 2, erzielt sie einen zusätzlichen Gewinn durch ihr Über-bzw. Untertreiben in der Höhe von

$$
\Delta \Pi:=\Pi^{22}\left(e_{1}^{i *}, e_{2}^{1 *}, z^{1 n}\right)-\Pi^{21}\left(e_{1}^{1 *}, e_{2}^{1 *}, z^{1 n}\right)
$$

Beginnt dagegen Regierung 2, gibt diese das für sie optimale erste Angebot $\mathrm{a}^{21} \mathrm{ab}$, und es kommt bei Typ 1 zur Aufteilung

$$
\left(\Pi^{1}\left(\mathrm{a}^{21}\right), \Pi^{21}\left(\mathrm{a}^{21}\right)\right)=\left(\frac{\left(1-\delta_{21}\right)_{1}}{1-\delta_{1} \delta_{21}} \mathrm{u}^{1 *}, \frac{1-\delta_{1}}{1-\delta_{1} \delta_{21}} \pi^{1 *}\right) .
$$

Ist Regierung 2 dagegen vom Typ 2, wäre für sie der zusätzliche Gewinn $\Delta \mathrm{n}$ der gleiche wie in (6.33).

Beginnt Regierung 1 die Verhandlungen und gibt ein Trennangebot $a^{15}$ $a b$, wenn q die Ungleichung (6.29) erfüllt, wird dieses entsprechend zu (6.22) durch

$$
\Pi^{22}\left(\mathbf{a}^{15}\right)=\Pi^{22}\left(\mathbf{a}^{21}\right) \delta_{: 22}
$$

bestimmt. Bezieht man (6.33) mit ein, läßt sich diese Bedingung umformen $\mathrm{zu}$

$$
\Pi^{22}\left(\mathbf{a}^{15}\right)=\left(\Pi^{21}\left(\mathbf{a}^{31}\right)+\Delta \Pi\right) \delta_{22}
$$

Falls Regierung 2 vom Typ 2 ist, lautet die entsprechende Gewinnaufteilung im Gleichgewicht bei einem Angebot $a^{1 s}$ 


$$
\left(\Pi^{1}\left(\mathrm{a}^{1 \mathrm{~s}}\right), \Pi^{2}\left(\mathrm{a}^{1 \mathrm{~s}}\right)\right)=\left(\mathrm{n}^{2 *}-\left(\frac{1-\delta_{1}}{1-\delta_{1} \delta_{21}} \pi^{1 *}+\Delta \pi\right) \delta_{22^{\prime}}\left(\frac{1-\delta_{1}}{1-\delta_{1} \delta_{21}} n^{1 *}+\Delta \pi\right) \delta_{22}\right),
$$

wobei $\mathrm{n}^{2 *}$ der maximale Effizienzgewinn in der Ökonomie sei, der bei Typ 2 realisierbar ist, $d . h$.

$$
\Pi^{2 *}=\Pi^{1}\left(e_{1}^{2 *}, e_{2}^{2 *}, z\right)+\Pi^{21}\left(e_{1}^{2 *}, e_{2}^{2 *}, z\right)
$$

Wenn dagegen Regierung 2 vom Typ 1 ist, kommt es nach entsprechender zeitlicher Verzögerung zur Aufteilung (6.34).Beginnt Regierung 2 die Verhandlungen und will eine Trennung verhindern, ist mit (6.25), (6.34) und (6.36) die entsprechende Aufteilung $\left.\left(\Pi^{1}\left(a^{2 n}\right)\right), \Pi^{21}\left(a^{2 n}\right)\right)$ festgelegt.

Durch die Aufteilungen der Effizienzgewinne (6.32), (6.34) und (6.36) sind gleichzeitig die Schranken (6.29) und (6.31) genau bestimmt.

Vereinfacht man das Beispiel und unterstellt keinerlei Differenzen in den physischen Umweltbedingungen zwischen den beiden Typen, d.h. es gilt $\Pi^{21}=\Pi^{22}=: \Pi^{2}$ und damit $\mathrm{II}^{1 *}=\Pi^{2 *}=\Pi^{*}$ sowie $\Delta_{\Pi}=0$, dann lassen sich auf sehr einfache Weise komparativ-statisch Veränderungen der Schranken (6.29) und (6.31) betrachten. Einsetzen der entsprechenden Effizienzgewinnanteile in (6.29) führt zu

$$
\mathrm{q}<\frac{1-\delta_{1}+\left(1-\delta_{1}\right)\left(\delta_{21}-\delta_{22}\right)-1+\delta_{21}}{1-\delta_{1}+\left(1-\delta_{1}\right)\left(\delta_{21}-\delta_{22}\right)-\delta_{1}^{2}\left(1-\delta_{21}\right)}
$$

Dieser Ausdruck läßt sich vereinfachen zu

$$
\mathrm{q}<\frac{\delta_{21}-\delta_{22}}{1-\delta_{22}+\delta_{1}\left(1-\delta_{21}\right)}
$$


Je größer der Unterschied zwischen den beiden möglichen Diskontfaktoren $\delta_{21}$ und $\delta_{22}$ von Regierung 2 ist, umso höher liegt der Wert dieser Schranke und damit der Anreiz der schlechter informierten Regierung 1, die beiden Typen von Regierung 2 voneinander zu trennen. Es ist aber auffallend, daß mit einer Verringerung der Zeitpräferenz von Regierung 1 $\left(\delta_{1}\right.$ steigt) der Wert der Schranke sinkt. Obwohl Regierung 1 "geduldiger" wird, verringert sich ihr Anreiz zur Trennung. Der Grund für dieses zunächst unerwartete Ergebnis liegt darin, da $\beta$ mit einer Verringerung der Zeitpräferenz ihre individuellen Kosten der Nichteinigung sinken. Damit erhält Regierung 1 einen höheren Anteil am Effizienzgewinn, gleichzeitig verringert sich aber dadurch auch der relative zusätzliche Gewinn, den sie erzielen könnte, wenn Regierung 2 vom Typ 2 ist.

Setzt man die entsprechenden Effizienzgewinnanteile in (6.31), so ergibt sich nach Vereinfachen des gewonnenen Ausdrucks

$$
q<\frac{\delta_{21}-\delta_{22}-\frac{1-\delta_{21}^{2}}{\delta_{1}}}{1-\delta_{22}+\delta_{1}\left(1-\delta_{21}\right)},
$$

Hierbei gilt ebenso, je größer der Unterschied zwischen den Diskontfaktoren $\delta_{21}$ und $\delta_{22}$ von Regierung 2 ist, umso eher besteht für Typ 1 ein Anreiz sich diskriminieren zu lassen. Verändert man komparativ-statisch den Diskontfaktor $\delta_{1}$ von Regierung 1 , sind keine eindeutigen Auswirkungen festzustellen.

\subsection{Zusammenfassung}

Das dynamisch-strategische Verhandlungsmodell mit unendlichem Zeithorizont wird in diesem Kapitel durch die Annahme einseitiger asymmetrischer Information zwischen den Regierungen erweitert. Die dadurch bei der schlechter informierten Regierung erzeugte Unsicherheit beeinflußt das beiderseitige strategische Verhalten im Verhandlungsprozeß. Bei der 
besser informierten Regierung löst diese Informationssituation Anreize aus, ihre Verhandlungsbedingungen in möglichst vorteilhafter Weise darzustellen, um Nutzenvorteile zu gewinnen. Die schlechter informierte Regierung besitzt indes den Anreiz, die wahren Bedingungen zu erfahren.

Im Modell wird zwischen zwei Ursachen von Informationsproblemen unterschieden, die unterschiedliche Motive zur Fehlinfomation enthalten. Zum einen kann durch Untertreiben der wahren Zeit- und Risikopräferenzen die relative Verhandlungsposition verbessert werden. Zum zweiten ist es möglicherweise vorteilhaft, durch Über- bzw. Untertreiben der tatsächlichen physischen Bedingungen in der eigenen Umweltregion, realisierbare Effizienzgewinnanteile vor der anderen Seite zu verbergen. Diese beiden Formen sind aufgrund der damit verbundenen Folgen unter theoretischen Gesichtspunkten unterschiedlich zu bewerten. Asymmetrische Information über die Präferenzen ist ausschließlich mit Verteilungswirkungen im Verhandlungsergebnis verbunden. ${ }^{11}$ Dagegen kann asymmetrische Information über die physischen Bedingungen zu Ineffizienzen in der ausgehandelten Umweltallokation führen.

Im Modell kann ein Informationstransfer ausschließlich endogen und indirekt über die Angebote während des Verhandlungsprozesses stattfinden. Möglichkeiten dazu bieten Angebote mit Selbstselektions- bzw. Signaleigenschaften, über welche die besser informierte Regierung ihre wahren Verhandlungsbedingungen indirekt offenbart. Eine solche Form der Informationsbeschaffung bzw. -verbreitung ist mit zusätzlichen individuellen Kosten verbunden, die durch zeitlich bedingte Verluste an Effizienzgewinnen und die Aufwendung zusätzlicher Ressourcen entstehen.

Die schlechter informierte Regierung muß der besser informierten Regierung eine Anreizprämie zahlen, die mindestens so hoch wie der

11) In dem strategischen Verhandlungsmodell von Fudenberg und Tirole (1983) mit ausschließlich asymmetrischer Information über Präferenzen entsteht allokative Ineffizienz, indem die Verhandlungsparteien uneinig auseinandergehen. Dieses Ergebnis ist aber nur durch eine exogene Beschränkung der maximalen Anzahl der Verhandlungsrunden möglich. 
Vorteil ist, den diese durch die unwahre Angabe der tatsächlichen Bedingungen im weiteren Verlauf der Verhandlungen erzielt hätte. Zeitliche Verzögerungen und damit Verluste an Effizienzgewinnen für beide Regierungen können auftreten, da der Informationstransfer im Sinne einer schrittweisen negativen Auslese von Verhandlungsrunde zu Verhandlungsrunde stattfindet und somit Zeit erfordert.

Dadurch entsteht ein Trade-off-Problem zwischen zwei verschiedenen Formen potentieller Transaktionskosten: Kosten der Nichteinigung und Kosten, um eine strategisch günstigere Position zu erreichen. Das Modell vereinfacht dieses Problem, indem es nur von zwei möglichen Ausprägungen der Verhandlungsbedingungen der besser informierten Regierung ausgeht.

Dieses Modell wird im Rahmen einer Gleichgewichtsanalyse untersucht, die wiederum auf dem nichtkooperativen Nash-Gleichgewichtskonzept aufbaut. Die spezielle Variante des teilspielperfekten Bayesschen NashGleichgewichts berücksichtigt neben der dynamischen Struktur des Verhandlungsprozesses zudem die Erwartungen, die sich die schlechter informierte Regierung über die tatsächlichen Verhandlungsbedingungen der anderen Regierung bildet.

Die gegenüber dem Modell in Kapitel 5 durch asymmetrische Information modifizierten Randbedingungen bewirken im Vergleich zu den dortigen Ergebnissen, daß im Gleichgewicht Ineffizienzen auftreten können. Es ist einerseits möglich, daß, anstatt sich sofort auf gesamtkostenminimale und damit paretooptimale Emissionsbeschränkungen zu einigen, eine solche Allokation erst nach einer zeitlichen Verzögerung zustande kommt. Fudenberg und Tirole (1983) bezeichnen dies als schwache Ineffizienz. Andererseits kann ebenso ohne Zeitverlust eine Einigung auf nicht paretooptimale Emissionsbeschränkungen erfolgen, welches im Sinne von Fudenberg und Tirole starker Ineffizienz entspricht. Im ersten Fall geht der Einigung immer ein Informationstransfer voraus, im zweiten dagegen nicht. 
Die Ursachen starker Ineffizienzen sind im einen Fall fehlende Anreize der schlechter informierten Regierung, zusätzliche Information über die andere Regierung zu gewinnen. Im anderen Fall bestehen für die besser informierte Regierung Anreize, einen zeitintensiven Informationstransfer zu vermeiden. In diesem Zusammenhang gilt als generelle Entscheidungsregel unter dem angenommenen Verhalten, da $\beta$ eine Regierung im Gleichgewicht nur in dem Maß Transaktionskosten im Sinne von Kosten der Informationsbeschaffung bzw. -verbreitung verursacht, soweit sich dadurch ihr erwarteter Nettonutzen erhöhen läßt.

Wie das Modell zeigt, trifft der erste Fall zu, wenn die schlechter informierte Regierung im Vergleich zur anderen eine relativ starke Verhandlungsposition besitzt und dadurch schon einen großen Anteil der unter den gegebenen Informationen realisierbaren Effizienzgewinne erhält. Der Anreiz, zusätzliche Information zu gewinnen, ist ebenfalls gering, wenn die schlechter informierte Regierung bei einer Trennung nur geringe zusätzliche Gewinne erwartet. Diese sind vor allem in den bei einer Fehlalloktion entstehenden Wohlfahrtsverlusten verborgen. Der zweite Fall ist nur in der Situation relevant, wenn die besser informierte Regierung die Verhandlungen beginnt. Je größer ihre putentiellen Kosten der Nichteinigung sind, umso eher ist sie bestrebt, einen Informationstransfer zu verhindern.

Eine ähnliche Überlegung gilt für den Anreiz der besser informierten Regierung, zu über- oder untertreiben. Hat diese aufgrund niedriger Kosten der Nichteinigung im Vergleich zur anderen Regierung eine starke relative Verhandlungsposition, verringert sich ihr Anreiz zur Fehlinformation, wenn sie dabei auch noch einen großen Teil des ansonsten entstandenen Wohlfahrtsverlusts für sich behalten kann. Das Trade-offProblem, welches zwischen dem Anreiz zur Fehlinformation und dem liegt, bei der Wahrheit zu bleiben, ist allerdings nicht explizit in der Gleichgewichtsanalyse betrachtet worden.

Ein weiterer Unterschied zu den Ergebnissen des Modells bei vollständiger Information wird deutlich, wenn beide Regierungen durch einen 
Informationstransfer einen zusätzlichen Nutzengewinn erzielen können. Der allgemeine Vorteil, die Stackelberg-Unabhängigkeitsposition einzunehmen, kehrt sich bei unvollständiger Information möglicherweise in einen Nachteil um. Beginnt die besser informierte Regierung die Verhandlungen, tritt durch den Informationsproze $B$ eine zusätzliche Zeitverzögerung ein, ohne die Verteilung der Effzienzgewinne letztendlich zu verändern. Beide Regierungen würden sich besserstellen, wenn die besser informierte Regierung der schlechter informierten die Möglichkeit des ersten Angebots einräumen würde. 



\section{Schlußbemerkung}

Ausgangspunkt der Untersuchung sind grenzüberschreitende Externalitäten, ausgelöst durch den zwischenstaatlich unkoordinierten, übermäßigen Einsatz von Allmende-Umweltgütern in inter- oder transnationalen ökologischen Systemen. Die überstaatliche Regulierung des Ressourceneinsatzes setzt - bedingt durch die nationalen Souveränitätsrechte - eine bilaterale oder multilaterale internationale Zusammenarbeit voraus. Der dazugehörige zwischenstaatliche marktliche Koordinierungsproze $B$ und ebenso die sich daran anschließenden administrativen Prozesse, mit denen die ausgehandelten Regelungen auf nationaler Ebene durchgesetzt werden müssen, sind für die betreffenden Staaten unter den jeweiligen Ausgangsbedingungen im unterschiedlichen $\mathrm{Ma} ß$ mit Transaktionskosten verbunden.

In diesem inhaltlichen Rahmen stellt die Arbeit einen theoretischen Beitrag dar, der zwischen der rein allokationstheoretisch ausgerichteten Umweltökonomie und der Institutionenökonomie angesiedelt ist. Sie beschreibt mit Hilfe von spieltheoretischen Ansätzen die Verhandlungssituation zwischen Staaten, die unter alternativen Formen von Regulierungseingriffen zu wählen haben. Dabei ist es das vorrangige Ziel der Arbeit, die Rolle der Transaktionskosten herauszuarbeiten, d.h. ihre wesentlichen Ursachen und ihre Auswirkungen auf das Zustandekommen sowie die Effizienzeigenschaften möglicher Verhandlungsergebnisse zu erklären. Damit knüpft die Untersuchung direkt an Überlegungen der Property-Rights-Lehre und des "Coase-Theorems" an.

Da die detaillierten Ergebnisse der einzelnen Kapitel schon jeweils an deren Schluß zusammengefaßt wurden, wollen wir an dieser Stelle die Gelegenheit nutzen, im Überblick die prinzipielle Bedeutung von Transaktionskosten zu diskutieren. Auf diese Weise werden am ehesten ökonomische Zusammenhänge deutlich, die vielleicht helfen, einen Teil der heute in der Wirklichkeit beobachteten Bestrebungen, aber auch die 
Schwierigkeiten bei der Lösung internationaler Umweltprobleme begründen zu können.

Der entscheidende Punkt in der Analyse von Transaktionskosten ist, daß sich diese weitgehend von Kosten im walrasianischen Sinn, wie beispielsweise Produktions- oder Transportkosten, unterscheiden. Transaktionskosten lassen sich weitgehend - natürlicherweise - nicht ex ante angeben. Beispielsweise in einem marktlichen Koordinierungsprozeß wird der überwiegende Teil implizit durch die Ausgangsbedingungen auf dem Verhandlungsmarkt, durch das Verhalten der Marktteilnehmer im Verlauf des Verhandlungsprozesses und durch ihr Verhalten danach bestimmt.

In der Arbeit wird der Einfluß von Transaktionskosten untersucht, indem ein solcher Koordinierungsprozeß mit potentiellen Transaktionskosten mittels dynamisch-strategischer Verhandlungsmodelle simuliert wird. Mit den Kosten der Nichteinigung und den Kosten für die Beschaffung nicht allseitig verfügbarer Information haben wir nur einen Teil der Ursachen von Transaktionskosten herausgegriffen, aber dieser ist für eine Analyse ihrer prinzipiellen Wirkungen zunächst ausreichend.

Zwei Ergebnisse sind hier von Bedeutung. Erstens sind positive potentielle Transaktionskosten einer Verhandlungspartei notwendig, um die im Ergebnis erreichte Allokation und Verteilung aus dem Modell heraus erklären zu können. Die potentiellen individuellen Transaktionskosten sind dabei Maß ihrer "Verhandlungsmacht". Zweitens impliziert das individuell-rationale Verhalten einer Verhandlungspartei, daß sie nur in dem Umfang Transaktionskosten verursacht, wie sich dadurch ihr erwarteter Nettonutzen steigern läßt.

Entscheidend für die Höhe der im Verhandlungsgleichgewicht realisierten Transaktionskosten sind die Informationsbedingungen in der Ausgangssituation. Die Modelle bei vollständiger Information, d.h. bei Sicherheit und allgemeiner Unsicherheit, haben gezeigt, daß eine Einigung auf ein nicht paretooptimales Ergebnis und das Hinauszögern einer Einigung 
im Sinne von Barzel (1985) "Ressourcenverschwendung" ist. Solange jeder Verhandlungsteilnehmer die Verhandlungsbedingungen der anderen genau kennt und weiß, wie sie auf bestimmte Aktionen reagieren, ist jedes "Feilschen" sinnlos.

Ein individuell-rationales Verhalten bei vollständiger Information impliziert folglich, daß in einem Verhandlungsgleichgewicht die potentiellen Transaktionskosten jeder Verhandlungspartei minimiert sind. In Kapitel 5, in dem als Verhandlungskosten ausschließlich der Verlust an Effizienzgewinnen bei Nichteinigung betrachtet wird, entstehen den Verhandlungsteilnehmern im Gleichgewicht daher keinerlei Kosten. Diese Überlegungen lassen sich entsprechend auf die Teile potentieller Transaktionskosten ausdehnen, die nach einer vertraglichen Einigung entstehen können.

Für den Fall vollständiger Information läßt sich somit der Schluß ziehen, da $\beta$ effiziente Verhandlungsergebnisse erzielt werden, solange die bei der Internalisierung von Externalitäten erwarteten Effizienzgewinne die verbleibenden (fixen) Transaktionskosten übersteigen.

Im Fall asymmetrischer Information dienen Transaktionskosten in der Form von Kosten der Informationsbeschaffung dazu, Anreize zur Fehlinformation zu kompensieren und dadurch zu vermeiden. Insofern werden sie aus individueller Sicht als "sinnvoller" Ressourceneinsatz in Kauf genommen. $\mathrm{Da}$ aber der im Modell mögliche Informationstransfer durch seine Kopplung an den Verhandlungsprozeß selbst Unvollkommenheiten aufweist, lassen sich die durch asymmetrische Information hervorgerufenen Ineffizienzen nicht vollständig beseitigen. In unserem Modell verhindern. unter Umständen die bei der Informationsgewinnung erwarteten Zeitverluste einen Transfer der Information.

Je nach Ausgangssituation kann ein effizientes Ergebnis, ein durch eine verzögerte Einigung auf eine paretooptimale Allokation schwach ineffizientes Ergebnis oder auch ein stark ineffizientes Ergebnis durch eine unverzögerte Einigung auf ein nicht paretooptimales Ergebnis erfolgen. 
Die Bestimmungsgrößen dafür sind vielfältiger Natur. Sie sind in den möglichen Bedingungen des Umweltsystems, den rechtlichen Ausgangsbedingungen, den Präferenzen und Erwartungen der Verhandlungsteilnehmer, wie auch in den Modalitäten des Verhandlungsprozesses enthalten.

Aus den Ergebnissen läßt sich im Gegensatz zu der weitverbreiteten Meinung in der Property-Rights-Lehre der Schluß ziehen, daß positive Transaktionskosten nicht per se die Ursache von Ineffizienzen sind. Aufwendungen, die im Modellbeispiel im Zusammenhang mit der Beschaffung von nicht allseitig verfügbarer Information entstehen, dienen gerade dazu, eine mögliche starke Ineffizienz zu vermeiden. ${ }^{\prime \prime}$

Das in Kapitel 5 und 6 entwickelte Grundmuster individuell-rationalen Verhaltens impliziert, daß entsprechend dem Nutzenkalkül verursachte Transaktionskosten auch immer mit einem Effizienzzuwachs verbunden sein müssen. Da transaktionskostenintensive Verteilungskämpfe als "Ressourcenverschwendung" vermieden werden, verursacht eine Verhandlungspartei nur dann Transaktionskosten, wenn sie durch entsprechende Ressourcenaufwendungen den absoluten Anteil an den Wohlfahrtsgewinnen erhöhen kann. Positive Wohlfahrtsgewinne ohne Verluste für die anderen Parteien sind aber grundsätzlich nur möglich, wenn sich durch die zusätzlichen Aufwendungen auch der gesamte Effizienzgewinn steigern läßt.

Die Idee dieses Verhaltensmusters läßt sich sicherlich auf weitere Informationsprobleme und andere Unvollkommenheiten marktlicher Koordinationsprozesse übertragen. Ebenso sind die in der Modellanalyse angestellten Überlegungen nicht nur auf das Beispiel grenzüberschreitender Umweltexternalitäten beschränkt, sondern können prinzipiell auf jegliches andere bi- oder multilaterale Verhandlungsproblem angewandt werden.

1) Die wahren Ursachen von Ineffizienzen in einer Situation mit asymmetrischer Information liegen dagegen in der Unvollkommenheit von Informationsprozessen, so daß unter Umstănden ein Informationstransfer nicht lohnend erscheint. 
Das neoklassische Grundmodell beschränkt jedoch die Beschreibung ausschließlich auf solche Ursachen von Transaktionskosten, die in den Ausgangs- und Umgebungsbedingungen von Verhandlungsmärkten enthalten sind. "Beschränkte Rationalität" oder "Opportunismus" der Teilnehmer von Koordinierungsprozessen, nach Williamson (1971), (1975) weitere Ursachen von Kosten dieser Art, lassen sich damit nicht untersuchen. 



\section{Literaturverzeichnis}

Alchian, A.A. und H. Demsetz (1972), Production, Information Costs, and Economic Organization, American Economic Review 62, 777-795.

d'Arge, R.C. (1974), Observations on the Economics of Transnational Environmental Externalities, in: OECD (Hrsg.), 147-175.

d'Arge, R.C. (1976), Transfrontier Pollution: Some Issues on Regulation, in: Walter, I. , Studies in Environmental Economics, 257-278.

Arnold, V. (1984), Umweltschutz als internationales öffentliches Gut: Komparative Kostenvorteile und Verhandlungsgewinne, Zeitschrift für Wirtschafts- und Sozialwissenschaften, 111-129.

Arrow, K.J. (1969), The Organization of Economic Activity: Issues Pertinent to the Choice of Market versus Nonmarket Allocation, in: Joint Economic Committee, The Analysis and Evaluation of Public Expenditure: The PPB-System, Washington D.C., 47-67.

Arrow, K.J. (1979), The Property Rights Doctrine and Demand Revealation under Incomplete Information, in: M. Boskin (Hrsg.), Essays in Honor of Tibor Scitovsky, New York, 23-39.

Asako, K. (1979), Environmental Pollution in an Open Economy, The Economic Record 55, 359-367.

Barzel, Y. (1985), Transaction Costs: Are They Just Costs, Zeitschrift für die gesamte Staatswissenschaft 141, 4-16.

Basta, D. J. und B.T. Bower (Hrsg.) (1982), Analyzing Natural Systems. Analysis for Regional Residuals-Environmental Quality Management.

Basta,D.J. und D.H. Moreau (1982), Introduction to Analyzing Natural Systems, in: D.J. Basta und B.T.Bower (Hrsg), 23-120. 
Baumol, W.J. und W.E. Oates (1975), The Theory of Environmental Policy: Externalities, Public Outlaws, and the Quality of Life, Englewood Cliffs, N. J.

Baumol, W.J. (1976), On International Issues in Environmental Management, in : Walter, I. (Hrsg.).

Binmore, K.G. (1980), Nash Bargaining Theory II, ICERD-Discussion Paper 80/14 London School of Economics.

Binmore, K.G. (1982), Perfect Equilibria in Bargaining Models, ICERDDiscussion Paper 82/58 London School of Economics.

Binmore, K.G., A. Rubinstein und A. Wolinsky (1985), The Nash Bargaining Solution in Economic Modelling, ICERD-Discussion Paper 85/112 London School of Economics.

Blair, R.D. und D.L. Kaserman (1983), Law and Economics of Vertical Integration and Control, New York.

Bonus, H. (1981), Emissionsrechte als Mittel der Privatisierung öffentlicher Ressourcen aus der Umwelt, in: L. Wegehenkel (Hrsg.), 54-77.

Bössmann, E. (1982), Volkswirtschaftliche Probleme der Transaktionskosten, Zeitschrift für die gesamte Staatswissenschaft 138, 664-679.

Braden, J.B. und D.W. Bromley (1981), The Economics over Collective Bads, Journal of Environmental Economics and Management 8, 134-150.

Breton, A. (1970), Public Goods and the Stability of Federalism, Kyklos 23, 882-902.

Buchanan, J. und C. Stubblebine (1962), Externality, Economica 29, 371-384. 
Campa, G. (1967), On the Pure Theory of Public Goods, Public Finance 21, 401-416.

Chatterjee, K. und W. Samuelson (1983), Bargaining under Incomplete Information, Operations Research 31, 835-851.

Coase, R. H. (1959), The Federal Communications Commission, Journal of Law and Economics 2, 1-40.

Coase, R. H. (1960), The Problem of Social Cost, Journal of Law and Economics 3, 1-44.

Conolly, M. (1972), Trade in Public Goods: A Diagrammatic Analysis, Quarterly Journal of Economics 86, 61-78.

Cramton, P.C. (1984), Bargaining with Incomplete Information: An Infinite Horizon Model with Two-Sided Uncertainty, Review of Economic Studies 51, 579-593.

Cramton, P.C. (1985), Continuous-Time Bargaining with Two-Sided Uncertainty, Yale School of Organization and Management, Working Paper Series D7.

Crawford ,V. (1982), A Theory of Disagreement in Bargaining, Econometrica 50, 607-638.

Dahlman, C.J. (1979), The Problem of Externality, Journal of Law and Economics 22, 141-162.

Damme, E. van (1983), Refinements of the Nash Equilibrium Concept, Berlin u.a..

Dasgupta, A.K. und D.W. Pearce (1972), Cost-Benefit-Analysis: Theory and Practice, London

Dasgupta, P., P. Hammond und E. Maskin (1979), The Implementation of Social Choice Rules: Some General Results on Incentive Compatibility, Review of Economic Studies 46, 185-216. 
Dasgupta, P., P. Hammond und E. Maskin (1980), On Imperfect Information and Optimal Pollution Control, Review of Economic Studies 47, 857-860.

Davis, O.A. und A. Whinston (1962), Externalities, Welfare, and the Theory of Games, Journal of Political Economy 70, 241-262.

Debreu, G. (1952), A Social Equilibrium Existence Theorem, Proceedings of the National Academy of Science 38, 886-893.

Demsetz, H. (1964), The Exchange and Enforcement of Property Rights, Journal of Law and Economics 7, 11-26.

Dixit, A. und V. Norman (1982), Außenhandelstheorie, München.

Elliott, D. und G. Yarrow (1977), Cost-Benefit Analysis and Environmental Policy: A Comment, Kyklos 30, 300-309.

Evans, A. (1978), Neighborhood Externalities, Economic Clubs, and the Environment, in: L. Wings und A. Evans (Hrsg.), Public Economics and the Quality of Life, Baltimore.

Ewe, T. (1986), Das Ozon-Drama, Bild der Wissenschaft Heft 6, 38-57.

Fay, J. A. und D. Golomb (1983), Controlling Acid Rain: Policy Issues, MIT Policy Discussion Series, MIT-EL 83-012

Fishburn, P.C. und A. Rubinstein (1982), Time Preference, International Economic Review 23, 719-736.

Forster, B.A. (1984), Economic Impact of Acid Precipitation: A Canadian Perspective, in: T.D. Crocker (Hrsg.), Economic Perspectives on Acid Deposition Control, Boston, 97-121.

Forsund, F.R. (1972), Allocation in Space and Environmental Pollution, Swedish Journal of Economics 74, 19-34.

Freeman III, A.M. (1979), The Benefits of Environmental Improvement: Theory and Practice, Baltimore. 
Friedman, J.W. (1977), Oligopoly and the Theory of Games, Amsterdam.

Fudenberg, D. und D. Levine (1983), Subgame-Perfect Equilibria of Finite- and Infinite-Horizon Games, Journal of Economic Theory 31, 251-268.

Fudenberg, D. und J. Tirole (1983), Sequential Bargaining with Incomplete Information, Review of Economic Studies 50, 221-247.

Furobotn, E.G. und S. Pejovich (1972), Property Rights and Economic Theory: A Survey of Recent Literature, Journal of Economic Literature $10,1137-1162$.

Gebauer, H. (1985), Regionale Umweltnutzungen in der Zeit. Eine intertemporale Zwei-Regionen-Analyse, Frankfurt.

Gladwin, T.N., J.L. Ugelow und I. Walter (1982), Approaches to International Negotiations on the Chlorofluorcarbon Problem, in: H. Siebert (Hrsg.), Global environmental Resources, Frankfurt, 1-55.

Green, J. und J.J. Laffont (1979), Incentives in Public Decision Making, Amsterdam.

Gronych, R. (1980), Allokationseffekte und Außenhandelswirkungen der Umweltpolitik, Tübingen.

Hahn, F. (1973), On Transaction Cost, Inessential Sequence Economics and Money, Review of Economic Studies 40, 449.

Harris, C. (1985), Existence and Characterization of Perfect Equilibrium in Games of Perfect Information, Econometrica 53, 613-628.

Harris M. und R.M. Townsend (1981), Resource Allocation under Asymmetric Information, Econometrica 49, 33-64.

Harsanyi, J. (1956), Approaches to the Bargaining Problem Before and After the Theory of Games, Econometrica 24, 144-156 
Harsanyi, J. (1967, 1968), Games with Incomplete Information Played by "Bayesian" Players, Part I-III, Management Science 14, 159-182, 320-334, 486-502.

Harsanyi, J.C. (1977), Rational Behavior and Bargaining Equilibrium in Games and Social Situations, Cambridge.

Harsanyi, J.C. und R. Selten (1972), A Generalized Nash Solution for Two-Person Bargaining Games with Incomplete Information, Management Science 18, 80-106.

Hart, O.D. (1983), Optimal Labour Contracts under Asymmetric Information: An Introduction, Review of Economic Studies 50, 3-35.

Hartje, V.J. (1984), Zur Effizienz von Institutionen der Meeresnutzung, Zeitschrift für die gesamte Staatswissenschaft 140, 712-736.

Heller, W.P. und D.A. Starrett, (1976), On the Nature of Externalities, in: Lin, S.A.Y. (Hrsg.), Theory and Measurement of Economic Externalities, New York

Hoffman, E. und M.L. Spitzer (1982), The Coase Theorem: Some Experimental Tests, Journal of Law and Economics 25, 73-98.

Hurwicz, L. (1972), On Informationally Decentralized Systems, in: R. Radner und B. McGuire (Hrsg.), Decision and Organization, Amsterdam, 297-336.

Jaffee, D. und T. Russell (1976), Imperfect Information, Uncertainty and Credit Rationing, Quarterly Journal of Economics 69, 651-666.

Johansen, L. (1982), On the Status of the Nash Type of Noncooperative Equilibrium in Economic Theory, Scandinavian Journal of Economics 84, 421-441.

Kalai, E. (1979), Nonsymmetric Nash Solutions and Replications of Two-Person Bargaining, International Journal of Game Theory 6, 129-133. 
Kiesling, H. (1976), A Model for Analyzing the Effects of Government Consolidation in the Presence of Public Goods, Kyklos 29, 233-255.

Kneese,A.V. und R.C. d'Arge (1984), Legal, Ethical, Economic and Political Aspects of Transfrontier Pollution, in: T.D. Crocker (Hrsg.), Economic Perspectives on Acid Deposition Control, Boston, 123-133.

Kneese, A. V. und M. Williams (1980), Environmental Aspects of Resource Policy in a Regional Setting: The Case of Air Quality in the Southwestern United States, in: Siebert, H. , I. Walter und K. Zimmermann (Hrsg.), 187-206.

Koopmans, T.C. (1960), Stationary Ordinal Utility and Impatience, Econometrica 28, 287-309.

Kosobud, R.F. und T.A. Daly (1984), Global Conflict or Cooperation over the $\mathrm{CO}_{2}$ Climate Impact, Kyklos 37, 638-659.

Krelle, W. (1976), Preistheorie, Tübingen.

Kurz, M. (1974), Equilibrium with Transaction Costs and Money in a Single Market Exchange, Journal of Economic 'Theory 7, 418.

Kwerel, E. (1977), To Tell the Truth: Imperfect Information and Optimal Pollution Control, Review of Economic Studies 44, 595-601.

Markusen, J.R. (1975a), Cooperative Control of International Pollution and Common Property Resources, Quarterly Journal of Economics 89, 618-632.

Markusen, J.R. (1975b), International Externalities and Optimal Tax Structures, Journal of International Economics 5, 15-29.

Mayer-Tasch, P.C. (1985), Die internationale Umweltpolitik als Herausforderung für die Nationalstaatlichkeit, in: Aus Politik und Zeitgeschichte, Beilage zur Wochenzeitung "Das Parlament", B 20/85. 
Minasian, J.R. (1975), Property Rights in Radiation: An Alternative Approach to Radio Frequency Allocation, Journal of Law and Economics 18, 221-272.

Mingst, K.A. (1982), Evaluating Public and Private Approaches to International Solutions to Acid Rain Pollution, Natural Resources Journal 22, 5-20.

Mishan, E.J. (1971), Cost-Benefit Analysis, London.

Mood, A.M., F.A. Graybill und D.C. Boes (1974), Introduction to the Theory of Statistics, 3. Aufl., New York.

Munro, G.R. (1979), The Optimal Management of Transboundary Renewable Resources, Canadian Journal of Economics 12, 355-376.

Muraro, G. (1974), The Economics of Unidirectional Transfrontier Pollution, in: OECD (Hrsg.), 33-74.

Muschett, F.D. (1982), Analyzing Atmospheric Systems, in: D.J. Basta und B.T. Bower (Hrsg.) 389-546.

Myerson, R.B. (1978), Refinements of the Nash Equilibrium Concept, International Journal of Game Theory 7, 73-80.

Myerson, R.B. (1979), Incentive Compatibility and the Bargaining Problem, Econometrica 47, 61-73.

Myerson, R.B. (1984), Two-Person Bargaining Problems with Incomplete Information, Econometrica 52, 461-488.

Myerson, R.B. und M. Satterthwaite (1983), Efficient Mechanisms for Bilateral Trading, Journal of Economic Theory 29, 265-281.

Nash, J.F. (1950), The Bargaining Problem, Econometrica 18, 155-162.

Nash, J.F. (1951), Noncooperative Games, Annals of Mathematics 54 , 286-295. 
Nash, J.F. (1953), Two-Person Cooperative Games, Econometrica 21, 128-140.

Niehans, J. (1975), Interest and Credit in General Equilibrium with Transaction Costs, American Economic Review 65, 548.

North, D.C. (1984), Transaction Costs, Institutions, and Economic History, Zeitschrift für die gesamte Staatswissenschaft 140, 7-12.

Okubo, A. (1980), Diffusion and Ecological Problems: Mathematical Models, Berlin u.a.

Olson, M. und R. Zeckhauser (1966), An Economic Theory of Alliances, Review of Economics and Statistics 48, 266-279.

Olson, M. und R. Zeckhauser (1970), The Efficient Production of External Economies, American Economic Review 60, 512-517.

Organisation for Economic Co-Operation and Development (Hrsg.) (1974), Problems in Transfrontier Pollution, Paris.

Organisation for Economic Co-Operation and Development (Hrsg.) (1976), Economics of Transfrontier Pollution, Paris.

Organisation for Economic Co-Operation and Development (Hrsg.) (1977), Legal Aspects of Transfrontier Pollution, Paris.

Organisation for Economic Co-Operation and Development (Hrsg.) (1981), Transfrontier Pollution and The Role of States, Paris.

Pauly, M.V. (1970), Optimality, Public Goods, and Local Governments: A Theoretic Analysis, Journal of Political Economy 78, 572-585.

Pearce, D.W. (1976), The Limits of Cost-Benefit Analysis as a Guide to Environmental Policy, Kyklos 29, 97-112.

Pearce, D.W. (1977), Cost-Benefit Analysis and Environmental Policy: A Reply to Elliott and Yarrow and to Smith, Kyklos 30, 314-318. 
Pethig, R. (1976), Pollution, Welfare, and Environmental Policy in the Theory of Comparative Advantage, Journal of Environmental Economics and Mangement 3, 160-169.

Pethig, R. (1982), Reciprocal Transfrontier Pollution, in: Siebert, H.

(Hrsg.) Global Environmental Resources: The Ozone Problem, Frankfurt.

Prittwitz, V. (1983a), Umwelt und Außenpolitik, in: Aus Politik und

Zeitgeschichte, Beilage zur Wochenzeitung "Das Parlament", B 42/83.

Prittwitz, V. (1984), Umweltaußenpolitik: Grenzüberschreitende Luftverschmutzung in Europa, Frankfurt.

Repullo,R. (1982), A Note on Imperfect Information and Optimal Pollution Control, Review of Economic Studies 49, 483-484.

Rest, A. (1978), Internationaler Umweltschutz und Haftung, Berlin.

Riley, J.G. und R. Zeckhauser (1983), Optimal Selling Strategies: When to Haggle and When to Hold Firm, Quarterly Journal of Economics 76, 267-287.

Riordan, M.H. (1984), Uncertainty, Asymmetric Information and Bilateral Contracts, Review of Economic Studies 51, 83-93.

Roberts, M.J. und A.M. Spence (1976), Effluent Charges and Licenses under Uncertainty, Journal of Public Economics 5, 193-208.

Rochet, J.-C. (1985), Bilateral Monopoly with Imperfect Information, Journal of Economic Theory 36, 214-236.

Roth, A.E. (1979), Axiomatic Models of Bargaining, Berlin u.a.

Roth, A.E.. (1985), A Note on Risk Aversion in a Perfect Equilibrium Model of Bargaining, Econometrica 53, 207-211.

Rothschild M. und J. Stiglitz (1976), Equilibrium in Competitive Insurance Markets, Quarterly Journal of Economics 69, 629-649. 
Rubinstein, A. (1982), Perfect Equilibrium in a Bargaining Model, Econometrica 50, 97-109.

Rubinstein, A. (1985), A Bargaining Model with Incomplete Information about Time Preferences, Econometrica 53, 1151-1172.

Rubinstein, A. und P.C. Fishburn (1982), Time Preference, International Economic Review 23, 677-694.

Der Rat von Sachverständigen für Umweltfragen (1983), Sondergutachten Waldschäden und Luftverunreinigungen, Stuttgart.

Samuelson, W. (1980), First Offer Bargains, Management Science 26, 155-164.

Samuelson, W. (1985), A Comment on the Coase Theorem, in: A. Roth (Hrsg.), Game theoretic Models of Bargaining, Cambridge, 321-339.

Sandler, T. (1975), Pareto Optimality, Pure Public Goods and Multiregional Spillovers, Scottish Journal of Political Economy 22, 25-38.

Sandler,T. und A.J. Culyer (1982a), Joint Products and Multijurisdictional Spillovers, Quarterly Journal of Economics 96, 707-716.

Sandler, T. und A.J. Culyer (1982b), Joint Products and Interjurisdictional Spillovers: Some Public Goods Geometry, Kyklos 35, 702-709.

Savage, L.J. (1954), The Foundations of Statistics, New York.

Scott, A. (1976), Transfrontier Pollution and Institutional Choice, in: Walter,I. (Hrsg.), Studies in Environmental Economics, 303-317.

Scott, A. (1980), International Environmental and Fisheries Pacts, in: T. Sandler, The Theory and Structures of International Political Economy, Boulder, 59-95. 
Selten, R. (1965), Spieltheoretische Behandlung eines Oligopolmodells mit Nachfrageträgheit, Zeitschrift für die gesamte Staatswissenschaft 121, 301-324.

Selten. R. (1975), Reexamination of the Perfectness Concept for Equilibrium Points in Extensive Games, International Journal of Game Theory 4, 25-55.

Shibata, H. (1971), A Bargaining Model of the Pure Theory of Public Expenditure, Journal of Political Economy 79, 1-29.

Shubik, M. (1982), Game theory in the Social Sciences: Concepts and Solutions, London.

Siebert, H. (1976), Analyse der Instrumente der Umweltpolitik, Göttingen.

Siebert, H. (1977), Environmental Quality and the Gains from Trade, Kyklos 30, 657-673.

Siebert, H. (1978), Ökonomische Theorie der Umwelt, Tübingen.

Siebert, H. (1979), Environmental Policy in the Two Country Case, Zeitschrift für Nationalökonomie 39, 259-274.

Siebert, H. (1981), Prak tische Schwierigkeiten bei der Steuerung der Umweltnutzung über Preise, in: L.Wegehenkel (1981), 28-53.

Siebert, H. (1983a), Spatial Aspects of Environmental Economics, Discussion-Paper No. 256-83, Universität Mannheim.

Siebert, H. (1983b), Ökonomische Theorie natürlicher Ressourcen, Tübingen.

Siebert, H., J. Eichberger, R. Gronych und R. Pethig (1980), Trade and Environment: A Theoretical Enquiry, Amsterdam.

Siebert, H., I. Walter und K. Zimmermann (1980), Regional Environmental Policy: The Economic Issues, New York. 
Smets, H. (1974), Alternative Economic Policies of Unidirectional Transfrontier Pollution, in: OECD, 1974, 75-146.

Smith, K.V. (1977), Cost-Benefit Analysis and Environmental Policy: A Comment, Kyklos 30, 310-313.

Sobel, J. und I. Takahashi (1983), A Multi-Stage Model of Bargaining, Review of Economic Studies 50, 411-426.

Spence, M. (1974), Market Signaling, Harvard.

Stahl, I. (1972), Bargaining Theory, Stockholm School of Economics Discussion Paper.

Storsberg, K.-H. und S. Ercman (1980), Auswertung der Erfahrungen mit den Methoden der Grenzüberschreitenden Zusammenarbeit auf nationaler, regionaler und örtlicher Ebene: Alternativvorschläge, Zeitschrift für Umweltpolitik 3.

Tiebout, C.M. (1956), A Pure Theory of Local Expenditures, Journal of Political Economy 64, 416-429.

Tietenberg, T.H. (1974), Derived Decision Rules for Pollution Control in a General Equilibrium Space Economy, Journal of Environmental Economics and Management 1, 3-16.

Tulkens, H. (1979), An Economic Model of International Negotiations Relating to Transfrontier Pollution, in: K. Krippendorff (Hrsg.), Communication and Control in Society, 199-212.

Vogt, S. (1981), Zur intertemporal wohlfahrtsoptimalen Nutzung knapper, natürlicher Ressourcen: Eine kontrolltheoretische Analyse, Tübingen.

Walter, I. (1975), International Economics of Pollution Control, New York. 
Walter, I. (Hrsg.) (1976), Studies in International Environmental Economics, New York.

Wegehenkel, L. (Hrsg.) (1981), Marktwirtschaft und Umwelt, Tübingen.

Weitzman, M.L. (1974), Prices vs. Quantities, Review of Economic Studies 41, 477-491.

Williams, A.(1966), The Optimal Provision of a Public Good in a System of Local Government, Journal of Political Economy 74, 18-33.

Williamson, O.E. (1971), The Vertical Integration of Production: Market Failure Considerations, American Economic Review 61, 112-123.

Williamson, O.E. (1975), Markets and Hierarchies: Analysis and Antitrust Implications, New York.

Windisch, R. (1981), Das Anreizproblem bei marktlicher Koordinierung der Nutzung knapper Umweltressourcen, in: L. Wegehenkel (Hrsg.), 105-149.

van Zele, R. (1978), An International Analytical Framework for Environmental Management, New York.

Zeuthen, F. (1930), Problems of Monopoly and Economic Warfare, London. 


\section{STAATLICHE ALLOKATIONSPOLITIK IM MARKTWIRTSCHAFTLICHEN SYSTEM}

Band 1 Horst Siebert (Hrsg.): Umweltallokation im Raum. 1982.

Band 2 Horst Siebert (Hrsg.): Global Environmental Resources. The Ozone Problem. 1982.

Band 3 Hans-Joachim Schulz: Steuerwirkungen in einem dynamischen Unternehmensmodell. Ein Beitrag zur Dynamisierung der Steuerüberwälzungsanalyse. 1981.

Band 4 Eberhard Wille (Hrsg.): Beiträge zur gesamtwirtschaftlichen Allokation. Allokationsprobleme im intermediären Bereich zwischen öffentlichem und privatem Wirtschafts. sektor. 1983.

Band 5 Heinz König (Hrsg.): Ausbildung und Arbeitsmarkt. 1983.

Band 6 Horst Siebert (Hrsg.): Reaktionen auf Energiepreissteigerungen. 1982.

Band 7 Eberhard Wille (Hrsg.): Konzeptionelle Probleme öffentlicher Planung. 1983.

Band 8 Ingeborg Kiesewetter-Wrana: Exporterlösinstabilität. Kritische Analyse eines entwicklungspolitischen Problems. 1982.

Band 9 Ferdinand Dudenhöffer: Mehrheitswahl-Entscheidungen über Umweltnutzungen. Eine Untersuchung von Gleichgewichtszuständen in einem mikroökonomischen Marktund Abstimmungsmodell. 1983.

Band 10 Horst Siebert (Hrsg.): Intertemporale Allokation. 1984.

Band 11 Helmut Meder: Die intertemporale Allokation erschöpfbarer Naturressourcen bei fehlenden Zukunftsmärkten und institutionalisierten Marktsubstituten. 1984.

Band 12 Ulrich Ring: Offentliche Planungsziele und staatliche Budgets. Zur Erfüllung öffentlicher Aufgaben durch nicht-staatliche Entscheidungseinheiten. 1985.

Band 13 Ehrentraud Graw: Informationseffizienz von Terminkontraktmärkten für Währungen. Eine empirische Untersuchung. 1984.

Band 14 Rüdiger Pethig (Ed.): Public Goods and Public Allocation Policy. 1985.

Band 15 Eberhard Wille (Hrsg.): Offentliche Planung auf Landesebene. Eine Analyse von Planungskonzepten in Deutschland, Osterreich und der Schweiz. 1986.

Band 16 Helga Gebauer: Regionale Umweltnutzungen in der Zeit. Eine intertemporale ZweiRegionen-Analyse. 1985.

Band 17 Christine Pfitzer: Integrierte Entwicklungsplanung als Allokationsinstrument auf Landesebene. Eine Analyse der öffentlichen Planung der Länder Hessen, Bayern und Niedersachsen. 1985.

Band 18 Heinz König (Hrsg.): Kontrolltheoretische Ansätze in makroökonometrischen Modellen. 1985.

Band 19 Theo Kempf: Theorie und Empirie betrieblicher Ausbildungsplatzangebote. 1985.

Band 20 Eberhard Wille (Hrsg.): Konkrete Probleme öffentlicher Planung. Grundlegende Aspekte der Zielbildung, Effizienz und Kontrolle. 1986.

Band 21 Eberhard Wille (Hrsg.): Informations- und Planungsprobleme in öffentlichen Aufgabenbereichen. Aspekte der Zielbildung und Outputmessung unter besonderer Berück. sichtigung des Gesundheitswesens. 1986.

Band 22 Bernd Gutting: Der Einfluß der Besteuerung auf die Entwicklung der Wohnungs- und Baulandmärkte. Eine intertemporale Analyse der bundesdeutschen Steuergesetze. 1986.

Band 23 Heiner Kuhl: Umweltressourcen als Gegenstand internationaler Verhandlungen. Eine theoretische Transaktionskostenanalyse. 1987.

Band 24 Hubert Hornbach: Besteuerung, Inflation und Kapitalallokation. Intersektorale und internationale Aspekte. 1987. 


